Portland State University

PDXScholar

\title{
Areal Extent and Volumes of the Dinner Creek Tuff Units, Eastern Oregon Based on Lithology, Bulk Rock Composition and Feldspar Mineralogy
}

Teresa Rae Hanna

Portland State University

Follow this and additional works at: https://pdxscholar.library.pdx.edu/open_access_etds

Part of the Geology Commons, and the Volcanology Commons Let us know how access to this document benefits you.

\section{Recommended Citation}

Hanna, Teresa Rae, "Areal Extent and Volumes of the Dinner Creek Tuff Units, Eastern Oregon Based on Lithology, Bulk Rock Composition and Feldspar Mineralogy" (2018). Dissertations and Theses. Paper 4346.

https://doi.org/10.15760/etd.6239

This Thesis is brought to you for free and open access. It has been accepted for inclusion in Dissertations and Theses by an authorized administrator of PDXScholar. Please contact us if we can make this document more accessible: pdxscholar@pdx.edu. 
Areal Extent and Volumes of the Dinner Creek Tuff Units, Eastern Oregon Based on Lithology, Bulk Rock Composition and Feldspar Mineralogy

by

Teresa Rae Hanna

A thesis submitted in partial fulfillment of the requirements for the degree of

Master of Science

in

Geology

Thesis Committee:

Martin J. Streck, Chair

Robert Perkins

John Bershaw

Portland State University

2018 


\begin{abstract}
The Dinner Creek Tuff erupted during a period of rhyolitic volcanism coeval to the flood volcanism associated with the Columbia River Basalt Group. The High Rock Caldera Complex, Lake Owyhee and McDermitt volcanic fields account for $~ 90 \%$ of the rhyolites erupted between 16.7-15.0 Ma. Situated at the northern end of the Lake Owyhee volcanic field, the Dinner Creek Tuff was originally mapped as a $\sim 2,000 \mathrm{~km}^{2}$ single ignimbrite confined to the Malheur Gorge. Streck et al. (2015) correlated tuff outcrops previously mapped as generic Miocene welded tuff as well as local units such as the "Mascall" or "Pleasant Valley" tuff of eastern Oregon to individual cooling units that comprise the newly redefined Dinner Creek Tuff, enclosing an area of $\sim 25,000 \mathrm{~km}^{2}$. Areal extents defined in this study show that all outcrops now determined to be Dinner Creek Tuff enclose an area of $\sim 31,800 \mathrm{~km}^{2}$ not including any fallout deposits that likely extended beyond the defined area.
\end{abstract}

Although Dinner Creek Tuff rhyolites have nearly identical compositions, different ages and subtle geochemical and mineralogical differences exist and were used to divide the Dinner Creek Tuff into four discrete cooling units. Except for unit 4, the units are lithologically very similar. Unit 1 is the Dinner Creek Tuff unit associated with the Malheur Gorge type section. The four cooling units have ages of 16.15-16 Ma (unit 1), 15.6-15.5 Ma (unit 2), 15.46 Ma (unit 3) and 15.0 Ma (unit 4).

Areal extents were established for all four cooling units based on feldspar compositions along with lithological and bulk rock geochemical data. Minimal extents of individual units are as follows: $\sim 22,590 \mathrm{~km}^{2}$ (unit 1), 17,920 $\mathrm{km}^{2}$ (unit 2), 14,170 km² 
(unit 3) and $~ 8,370 \mathrm{~km}^{2}$ (unit 4). Using conservative thicknesses, determined erupted tuff volumes are $\sim 170 \mathrm{~km}^{3}$ (unit 1), $125 \mathrm{~km}^{3}$ (unit 2), $\sim 99 \mathrm{~km}^{3}$ (unit 3) and $\sim 46 \mathrm{~km}^{3}$ (unit 4), totaling $\sim 440 \mathrm{~km}^{3}$ and dense rock equivalents are $\sim 152 \mathrm{~km}^{3}$ (unit 1), $\sim 96 \mathrm{~km}^{3}$ (unit 2), $\sim 76 \mathrm{~km}^{3}$ (unit 3) and $\sim 31 \mathrm{~km}^{3}$ (unit 4), totaling $\sim 356 \mathrm{~km}^{3}$.

These extents and volumes are the absolute minimum based solely on the locations of exposed tuff sections and the inclusion of the source. Centering eruptive units on source areas where they are known, expands the tuff extents into a more radial pattern as would be expected for low-aspect ratio, high energy ash-flow tuff eruptions. These probable extents increase the areal extents of the individual units to: $\sim 36,900 \mathrm{~km}^{2}$ (unit 1), $\sim 31,660 \mathrm{~km}^{2}$ (unit 2), 17,290 $\mathrm{km}^{2}$ (unit 3) and $\sim 10,150 \mathrm{~km}^{2}$ (unit 4) distributed over a $\sim 43,490 \mathrm{~km}^{2}$ area. Likewise, erupted tuff volume and dense rock equivalents also increase: volume - $277 \mathrm{~km}^{3}$ (unit 1), $\sim 222 \mathrm{~km}^{3}$ (unit 2), $\sim 121 \mathrm{~km}^{3}$ (unit 3) and $\sim 56$ $\mathrm{km}^{3}$ (unit 4); DRE— $248 \mathrm{~km}^{3}$ (unit 1), $\sim 170 \mathrm{~km}^{3}$ (unit 2), $\sim 93 \mathrm{~km}^{3}$ (unit 3) and $\sim 38 \mathrm{~km}^{3}$ (unit 4).

New mapping confirms previous hypotheses that the Castle Rock caldera erupted unit 1 and identified the new Ironside Mountain caldera as the source for unit 2 while precise source areas for unit 3 and 4 are not yet known but are thought to lie within the Dinner Creek Eruptive Center. Minimal calculated caldera volumes for units 1 and 2 are $\sim 98.5 \mathrm{~km}^{3}$ (unit 1) and $\sim 31.1 \mathrm{~km}^{3}$ (unit 2). Adding the thick ponded intra caldera tuff volume to the determined and probable erupted tuff volumes determined in this study, increases the erupted volumes to $\sim 268 \mathrm{~km}^{3}$ (determined) and $\sim 375 \mathrm{~km}^{3}$ (probable) for unit 1 along with $\sim 157 \mathrm{~km}^{3}$ (determined) and $\sim 253 \mathrm{~km}^{3}$ (probable) for unit 2. DREs 
increase to $\sim 251 \mathrm{~km}^{3}$ (determined) and $\sim 347 \mathrm{~km}^{3}$ (probable) for unit 1 along with $\sim 128$ $\mathrm{km}^{3}$ (determined) and $\sim 202 \mathrm{~km}^{3}$ (probable) for unit 2 . 


\section{ACKNOWLEDGEMENTS}

I would like to thank my advisor Martin Streck for his expertise, guidance and patience as well as my committee-Martin Streck, John Bershaw and Robert Perkins for their time and efforts reviewing my work. This study was possible due to support from the National Science Foundation grant EAR-1551495 to Martin Streck. I greatly appreciate the work of my fellow graduate and undergraduate students that providing geochemical data and/or samples used in in this study. Lastly, I would like to thank my family and friends for their continued support and encouragement throughout my academic career. 


\section{TABLE OF CONTENTS}

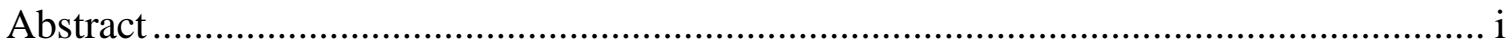

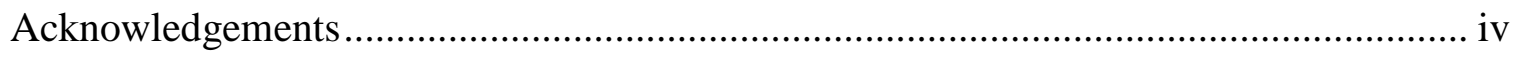

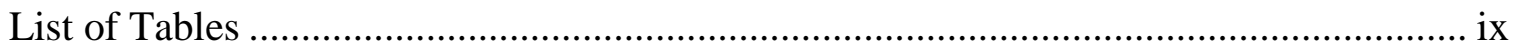

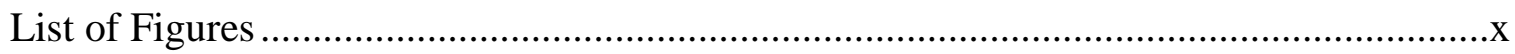

List of Abbreviations .......................................................................................... xviii

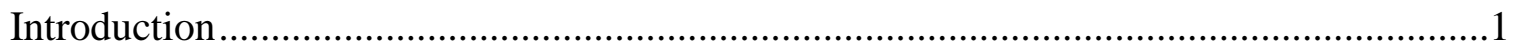

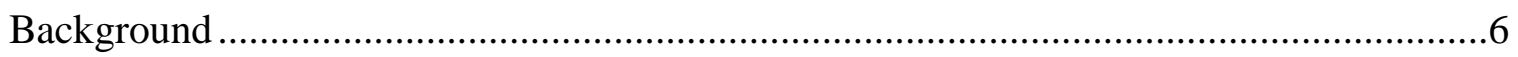

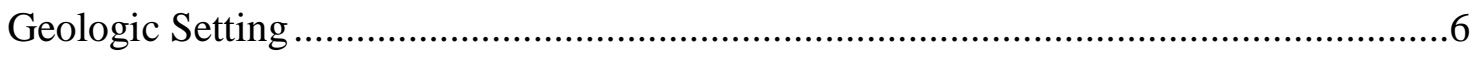

Blue Mountain Province and Accreted Terranes....................................................6

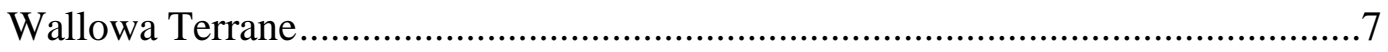

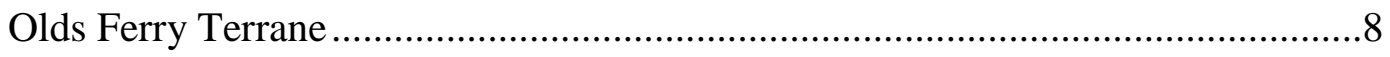

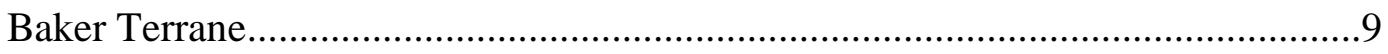

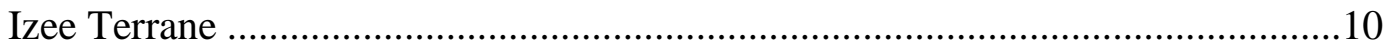

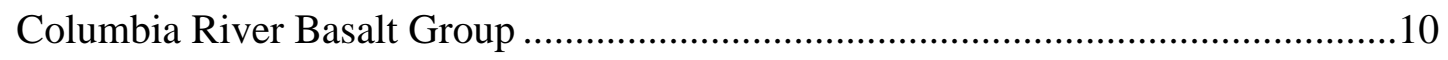

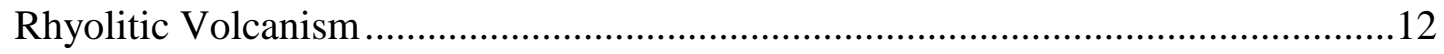

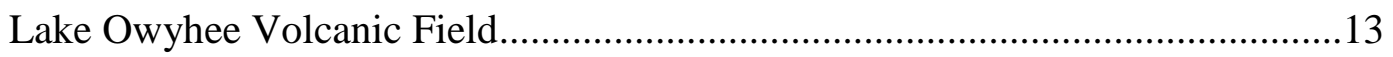

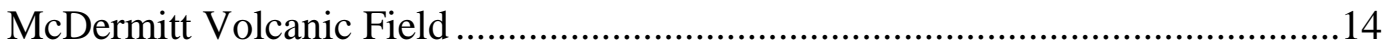

High Rock Caldera Complex ............................................................... 15

Snake River Plain/Yellowstone Hotspot Trend ............................................16

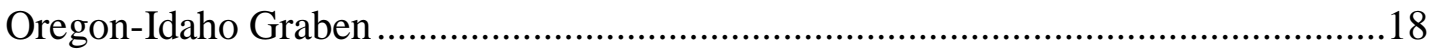

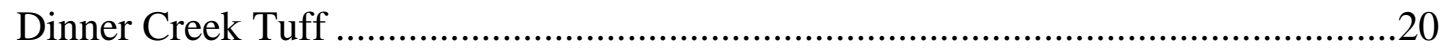




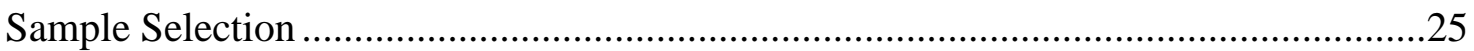

Chemical Analysis and Unit Designation ........................................................26

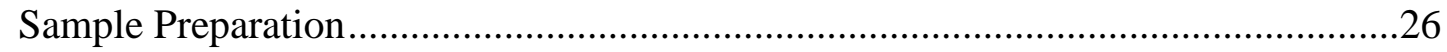

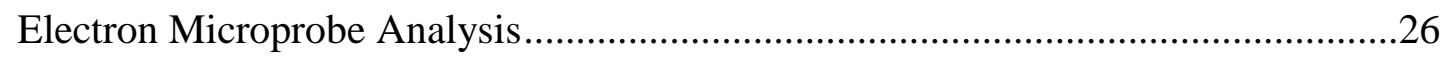

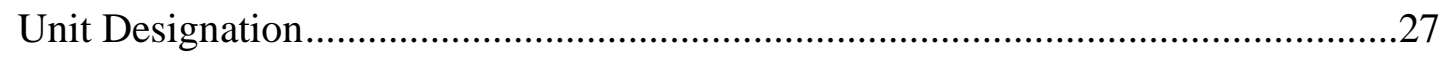

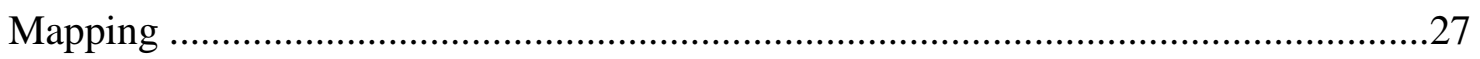

Tuff Volumes and Dense Rock Equivalent ..........................................................28

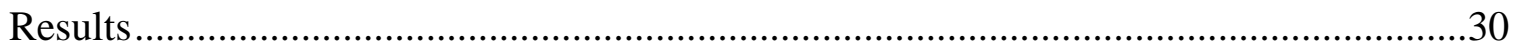

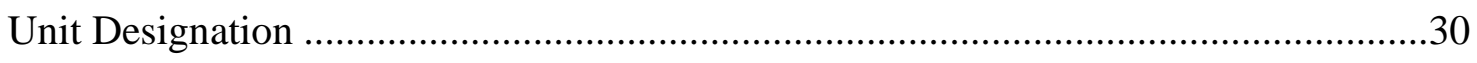

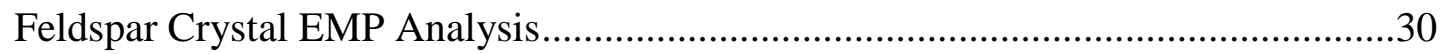

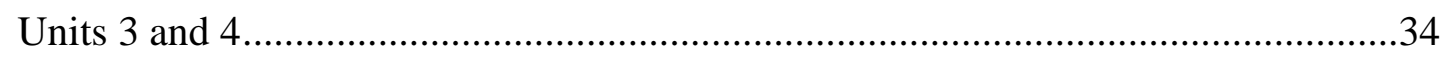

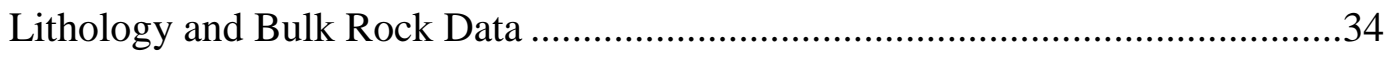

Unit Designating Feldspar Major Element Chemistry ..........................................35

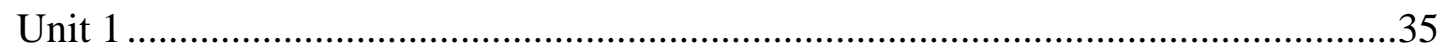

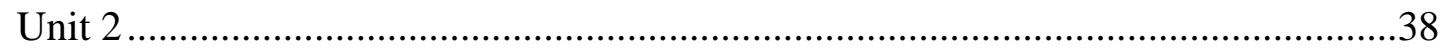

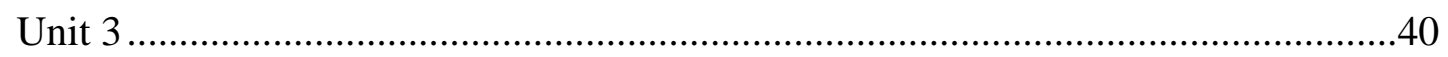

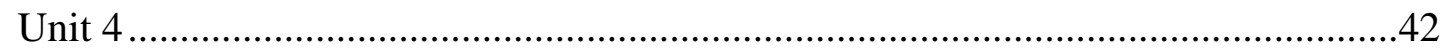

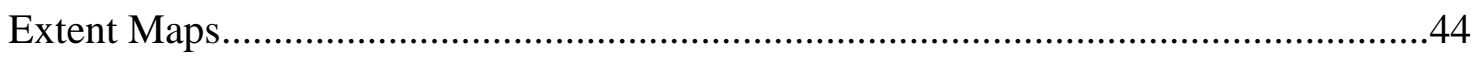

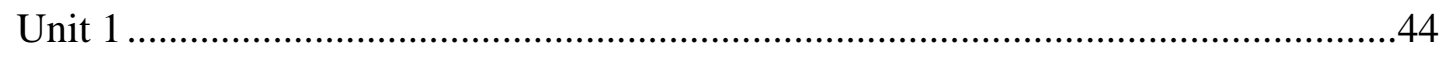

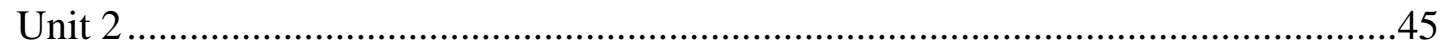

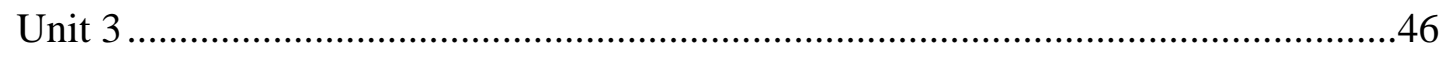

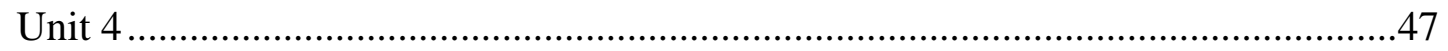


Total Extent .48

Non-unit Designating Felspars ...........................................................................49

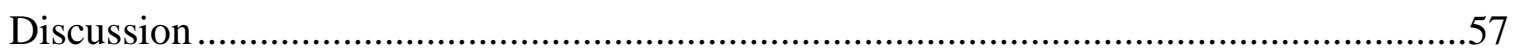

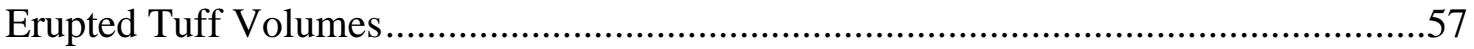

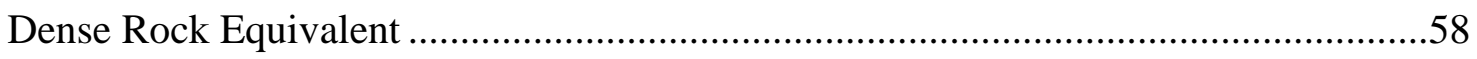

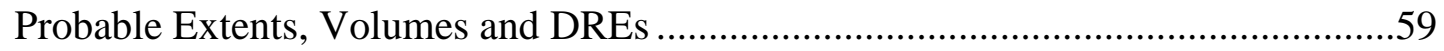

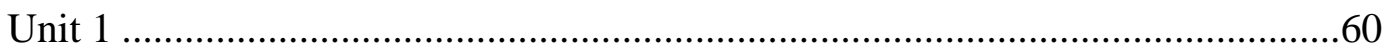

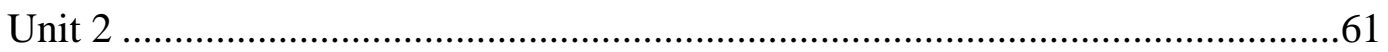

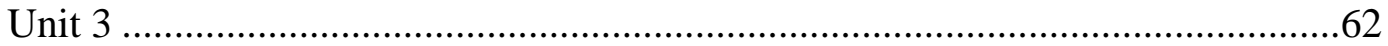

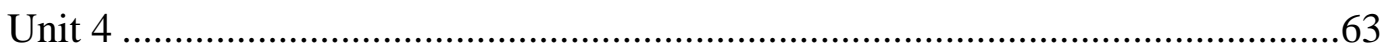

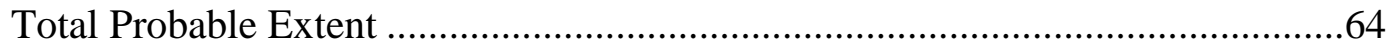

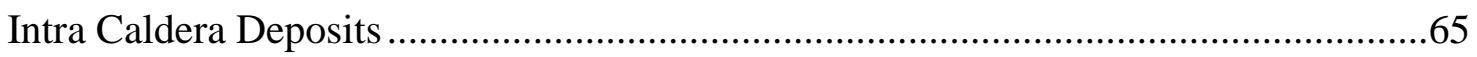

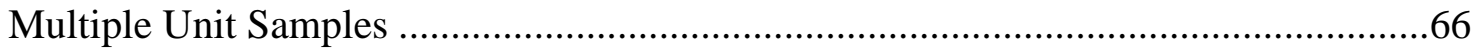

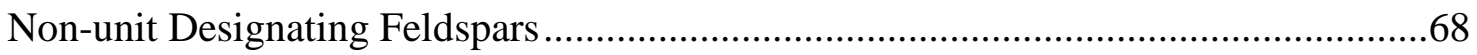

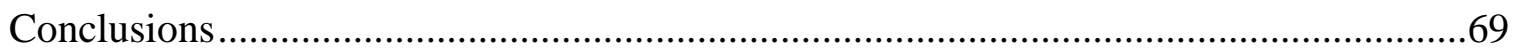

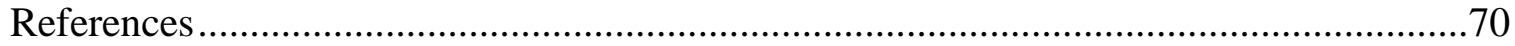

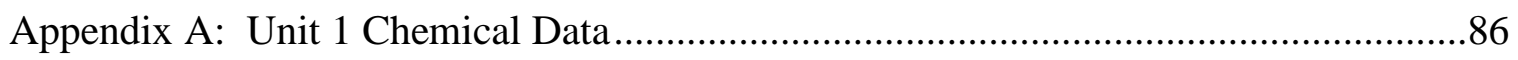

Unit 1 Average End Member Concentrations, Standard Deviations and GPS

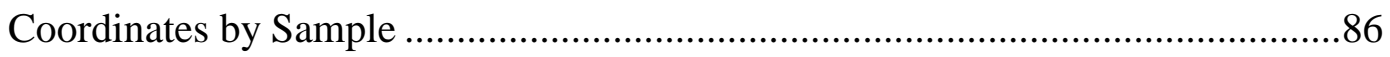

Unit 1 Individual Felspar Major Element Crystal Chemical Data .............................86

Unit 1 Individual Felspar Crystal End Member Compositions ...................................92

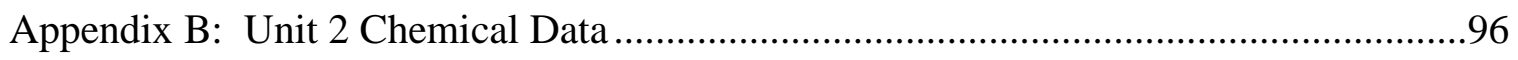

Unit 2 Average End Member Concentrations, Standard Deviations and GPS 
Coordinates by Sample .96

Unit 2 Individual Felspar Major Element Crystal Chemical Data .97

Unit 2 Individual Felspar Crystal End Member Compositions 100

Appendix C: Unit 3 Chemical Data

Unit 3 Average End Member Compositions, Standard Deviations and GPS Coordinates by Sample. .103

Unit 3 Individual Felspar Major Element Crystal Chemical Data 104

Unit 3 Individual Felspar Crystal End Member Compositions .108

Appendix D: Unit 4 Chemical Data

Unit 4 Average End Member Compositions, Standard Deviations and GPS Coordinates by Sample 112

Unit 4 Individual Felspar Major Element Crystal Chemical Data 113

Unit 4 Individual Felspar Crystal End Member Compositions 114

Appendix E: Non-unit Designating Felspar Chemical Data 117

Non-unit Designating Individual Felspar Major Element Crystal Chemical Data ......117

Unit 2 Individual Felspar Crystal End Member Compositions ...............................121

Appendix F: Bulk Rock Chemical Data .........................................................125

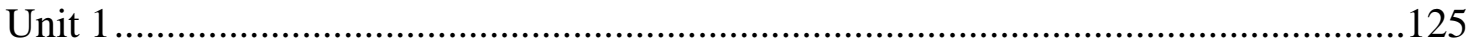

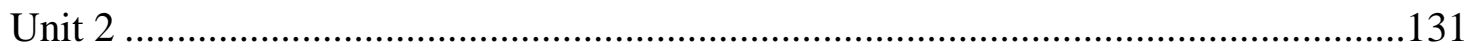

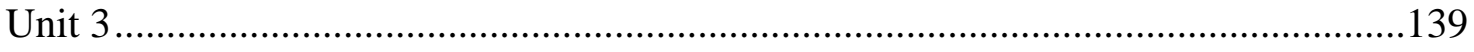

Unit 4 .141 


\section{LIST OF TABLES}

Table 1. Average feldspar end member component composition statistics by unit. The table includes maximums, minimums, averages and standard deviations for anorthite, albite and orthoclase for each unit

Table 2. Bulk tuff $\mathrm{SiO}_{2}$ concentrations for five samples, sample color for all samples correlated to either unit 3 or 4

Table 3. Average major element and barium feldspar geochemistry collected during this study for unit 1 correlated samples .36

Table 4. Average major element and barium feldspar geochemistry collected during this study for unit 2 correlated samples

Table 5. Average major element and barium feldspar geochemistry collected during this study for unit 3 correlated samples

Table 6. Average major element and barium feldspar geochemistry collected during this study for unit 4 correlated samples

Table 7. Unit thicknesses for volume calculations. .58

Table 8. Outcrop thicknesses observed during sample collection proximal to sample location .58

Table 9. Unit densities and DREs. .58

Table 10. Approximate determined and probable areal extents, volumes and DREs ......60

Table 11. Caldera observed thicknesses and axis used in minimal volume calculations .65

Table 12. Approximate determined and probable volumes and DRE including caldera deposits. .66 


\section{LIST OF FIGURES}

Figure 1. Regional overview map modified from Benson and Mahood (2016). Included are the Columbia River Basalt Group member extents after Reidel et al. (2013) and the generalized locations of feeder dikes after Tolan et al. (1989) and Camp et al. (2003). Red circle and lines delineate age progression of rhyolites in the High Lava Plains/Newberry Trend from 0 to 10 Ma. Black lined fields indicate approximate locations of the High Rock Caldera Complex, McDermitt and Lake Owyhee volcanic fields. Blue outlines indicate approximate locations of 16.4 to 0.6 Ma silicic centers associated with the Snake River Plain-Yellowstone trend after Smith et al. (2009). Purple line- $0.706{ }^{87} \mathrm{Sr}^{86}{ }^{86} \mathrm{Sr}_{\mathrm{i}}$ isopleths and pink line$0.706{ }^{87} \mathrm{Sr} /{ }^{86} \mathrm{Sr}_{i}$ isopleths are drawn using data from Armstrong et al. (1977), Farmer and DePaolo (1983), Fleck and Criss (1985), Criss and Fleck (1987), Kistler and Lee (1989), Elison et al. (1990), Leeman et al. (1992), Manduca et al. (1992), Wooden et al. (1999), Evans et al. (2002), Fleck and Criss (2007), Unruh et al. (2008), Pierce and Morgan, (2009) and Gaschnig et al. (2011)......................2

Figure 2. A and B: Regional overview, extent of the Dinner Creek Tuff and locations of correlative fallout units from Streck et al. (2015). A: Yellow frame is areal coverage of C. Stars indicate fallout localities: M-Mascall Formation, PBPaulina Basin, BU—Bully Creek Formation, SC—Succor Creek (after Nash and Perkins, 2012), UM—Umatilla (after Ferns, personal data). B: Stars indicate regional fallout localities after Nash and Perkins (2012): NV—Nevada, BCBuffalo Canyon, C—Carlin, VV—Virgin Valley, ID—Idaho, WB—White Bird; WA —Washington; A—Asotin. Dark and light blue — CRBG after Camp and 
Ross, (2004); red—extent of 17-12 Ma rhyolites after Christiansen et al. (2002).

C: Orange dots—-investigated Dinner Creek Tuff locations; red-database of

Oregon Department of Geology and Mineral Industries showing original Dinner

Creek Tuff and unnamed tuffs correlated with Dinner Creek Tuff; thick orange line — envelope around most distal outcrops; dashed orange oval—likely source area of Dinner Creek Tuff eruptive center (DITEC); yellow-original extent of Dinner Creek Welded Tuff and previous generalized extents of local ignimbrites here correlated with Dinner Creek Tuff; Abbreviations: MG- Malheur Gorge, CR - Castle Rock, H - town of Harper, IS - Ironside Mountains, J-town of Juntura, PG_-Picture Gorge, PB —-Paulina Basin; T—tuff. The black triangle outlines represent the approximate locations of Castle Rock and Ironside Mountain, the confirmed eruption sites for units 1 and 2, respectively

Figure 3. Simplified geologic of the Blue Mountains Province (BMP) showing preCenozoic geology after Dorsey and LaMaskin (2008), including the ages and extents of terranes that comprise the BMP

Figure 4. Major subdivisions and ages of the Columbia River Basalt Group from Reidel et al. (2013)

Figure 5. Track of the Yellowstone hotspot (Y) from Smith et al. (2009) indicating the relative motion of age-transgressive ESRP silicic volcanic centers (Armstrong et al., 1975; Smith and Braile, 1984; Pierce and Morgan, 1990, 1992; Kuntz et al., 1992; Smith and Braile, 1994; Perkins and Nash, 2002) opposite to the direction of North America plate motion (large arrow) (Smith and Sbar, 1974). Green outline is the topographically low area occupied by the Snake River Plain. 
Yellow outlines are post-17 Ma silicic volcanic centers containing multiple caldera-forming eruptions (Christiansen, 1984, 2001). Columbia River flood basalts are gray (Perkins and Nash, 2002). Red dots are historic earthquake epicenters taken from compilations of the University of Utah of M1.5-7.5 earthquakes. Black lines show late Quaternary faults; Cenozoic basaltic dikes are shown in yellow and orange (Zoback and Thompson, 1978; Camp, 1995; Glen and Ponce, 2002). ${ }^{87} \mathrm{Sr} /{ }^{86} \mathrm{Sr}$ isotope boundary for the 0.706 values is shown as a black-dashed line and is interpreted to separate continental lithosphere to the east and oceanic lithosphere to the west (Armstrong et al., 1977; Farmer and DePaolo, 1983). The tectonic parabola is defined by the bow shaped pattern of high topography and seismicity surrounding the YSRP (yellow dashed lines) (Smith et al., 1985; Smith and Arabasz, 1991; Anders and Sleep, 1992). The Newberry trend of silicic volcanism extends northwest across southeast Oregon to the Newberry caldera (N) (MacLeod et al., 1976)

Figure 6. Regional map showing the Oregon-Idaho graben (OIG) in context of the dike swarms of the Columbia River Basalt Group (CRBG dikes), the northern Nevada rift (NNR), and the western Snake River Plain (WSRP) after Cummings et al. (2000). Regional features near the Oregon-Idaho graben depicted. Numbers displayed along the Snake River Plain (SRP) show the age progression of rhyolitic volcanism. 19

Figure 7. Age relationships of ignimbrite and fallout tuffs originating from the Dinner 
Creek Tuff eruptive center in relation to eruption period of Grande Ronde Basalt lavas based on the Barry et al. (2013) and Jarboe et al. (2010) chronology after

Streck et al. (2015)

Figure 8. Dinner Creek Tuff unit photographs by Martin Streck. A1: Unit 1 type section in Malheur Gorge. A2: Unit 1 sample with white pumices. B1: Unit 2 outcrop east of John Day, OR. B2: Unit 2 near John Day, OR. C1: Unit 3 outcrop previously identified as the Bully Creek Tuff. C2: Unit 3, the recently correlated Bully Creek Tuff. D1: Unit 4 outcrop. D2: Unit 4 with vesicular globules of tholeiitic andesite glass that resemble Hunter Creek Basalt (Streck et al., 2015) .21

Figure 9. $\mathrm{Sr}, \mathrm{Y}, \mathrm{FeO}$ and $\mathrm{TiO}_{2}$ vs. $\mathrm{SiO}_{2}$ for all analyzed samples of Dinner Creek Tuff. Unit correlation based on feldspar composition, subtle chemical differences and available age dates

Figure 10. Anorthite vs. orthoclase diagram indicating unit correlating feldspar compositions from this study

Figure 11. Sample locations for this study. Castle Rock and Ironside Mountain are the eruption sites for units 1 and 2, respectively. They also mark the north/south boundaries of the DITEC.

Figure 12. Example of how each polygon was created to enclose the sample locations while including Castle Rock (unit 1 source), Ironside Mountain (unit 2 source) or the DITEC from Figure 2, but not centralizing the eruptive sources within the individual polygons. Solid vs. hollow shapes differentiate data sources

Figure 13. Sample locations for this study. Blue dots refer to samples that yielded 
sufficient mineral chemical data for unit correlation. Red dots refer to samples that either lacked feldspar for geochemical analysis or analysis indicated non-unit designating feldspars. Castle Rock and Ironside Mountain are the eruption sites for units 1 and 2, respectively. They also mark the north/south boundaries of the DITEC

Figures 14a and 14b. Anorthite vs. orthoclase diagram correlating samples in this study to the cooling units

Figure $15 . \mathrm{Nb}$ and $\mathrm{Sr}$ vs. $\mathrm{SiO}_{2}$ diagrams from the bulk rock data also differentiate unit 3 from unit 4 .35

Figure 16. Areal extent of the Dinner Creek Tuff unit 1. Left: Sample names and locations utilized in determining the extent. Open symbols represent samples from Streck et al. (2015) while solid symbols represent samples from this study. Right: Overall areal extent of unit 1 covers an area of $\sim 22,590 \mathrm{~km}^{2}$. Castle Rock and Ironside Mountain are the eruption sites for units 1 and 2, respectively. They also mark the north/south boundaries of the DITEC.

Figure 17. Areal extent of the Dinner Creek Tuff unit 2. Left: Sample names and locations utilized in determining the extent. Open symbols represent samples from Streck et al. (2015) while solid symbols represent samples from this study. Right: Overall areal extent of unit 2 covers an area of $\sim 17,920 \mathrm{~km}^{2}$. Castle Rock and Ironside Mountain are the eruption sites for units 1 and 2, respectively. They also mark the north/south boundaries of the DITEC.

Figure 18. Areal extent of the Dinner Creek Tuff unit 3. Left: Sample names and 
locations utilized in determining the extent. Open symbols represent samples from Streck et al. (2015) while solid symbols represent samples from this study. Right: Overall areal extent of unit 3 covers an area of $\sim 14,170 \mathrm{~km}^{2}$. Castle Rock and Ironside Mountain are the eruption sites for units 1 and 2, respectively. They also mark the north/south boundaries of the DITEC.

Figure 19. Areal extent of the Dinner Creek Tuff unit 4. Left: Sample names and locations utilized in determining the extent. Open symbols represent samples from Streck et al. (2015) and correlated samples collected by Aaron Steiner while solid symbols represent samples from this study. Right: Overall areal extent of unit 4 covers an area of $\sim 8,370 \mathrm{~km}^{2}$. Castle Rock and Ironside Mountain are the eruption sites for units 1 and 2, respectively. They also mark the north/south boundaries of the DITEC.

Figure 20. Overall areal extent of the Dinner Creek Tuff covers an area of $\sim 31,300$ $\mathrm{km}^{2}$. Castle Rock and Ironside Mountain are the eruption sites for units 1 and 2, respectively. They also mark the north/south boundaries of the DITEC. .48

Figure 21. Non-unit designation feldspar endmember concentration diagram for samples associated with unit 1

Figure 22a-c. Non-unit designation feldspar endmember concentration diagram for samples associated with unit 2 .

Figure 23. Non-unit designation feldspar endmember concentration diagram for samples associated with unit 3 . .54 
Figure 24. Non-unit designation feldspar endmember concentration diagram for samples associated with unit 4 .55

Figure 25. Non-unit designation feldspar endmember concentration diagram for samples not correlated to a unit .56

Figure 26. Probable areal extent of the Dinner Creek Tuff unit 1. Left: Sample names and locations utilized in determining the extent. Open symbols represent samples from Streck et al. (2015) while solid symbols represent samples from this study. Right: Solid shape-overall areal extent of unit 1 covers an area of $\sim 22,590 \mathrm{~km}^{2}$. Dashed line_- probable extent covering an area of $\sim 36,900 \mathrm{~km}^{2}$.

Castle Rock and Ironside Mountain are the eruption sites for units 1 and 2, respectively. They also mark the north/south boundaries of the DITEC

Figure 27. Probable areal extent of the Dinner Creek Tuff unit 2. Left: Sample names and locations utilized in determining the extent. Open symbols represent samples from Streck et al. (2015) while solid symbols represent samples from this study. Right: Solid shape-overall areal extent of unit 2 covers an area of $\sim 17,920 \mathrm{~km}^{2}$. Dashed line_-probable extent covering an area of $\sim 31,660 \mathrm{~km}^{2}$. Castle Rock and Ironside Mountain are the eruption sites for units 1 and 2, respectively. They also mark the north/south boundaries of the DITEC. .61

Figure 28. Probable areal extent of the Dinner Creek Tuff unit 3. Left: Sample names and locations utilized in determining the extent. Open symbols represent samples from Streck et al. (2015) while solid symbols represent samples from this study. Right: Solid shape_-overall areal extent of unit 3 covers an area of $\sim 14,170 \mathrm{~km}^{2}$. Dashed line_-probable extent covering an area of $\sim 17,290 \mathrm{~km}^{2}$. Castle Rock and 
Ironside Mountain are the eruption sites for units 1 and 2, respectively. They also mark the approximate north/south boundaries of the DITEC

Figure 29. Probable areal extent of the Dinner Creek Tuff unit 4. Left: Sample names and locations utilized in determining the extent. Open symbols represent samples from Streck et al. (2015) and correlated samples collected by Aaron Steiner while solid symbols represent samples from this study. Right: Solid shape - overall areal extent of unit 4 covers an area of $\sim 8,370 \mathrm{~km}^{2}$. Dashed line - probable extent covering an area of $\sim 10,150 \mathrm{~km}^{2}$. Castle Rock and Ironside Mountain are the eruption sites for units 1 and 2, respectively. They also mark the approximate north/south boundaries of the DITEC

Figure 30. Solid shape-Overall areal extent of the Dinner Creek Tuff covers an area of $\sim 31,800 \mathrm{~km}^{2}$. Dashed line_-overall probable areal extent covers an area of $\sim 43,490 \mathrm{~km}^{2}$. Castle Rock and Ironside Mountain are the eruption sites for units 1 and 2, respectively. They also mark the north/south boundaries of the DITEC.

Figure 31a and 31b. Anorthite vs. orthoclase diagram with single crystal anomalies that correlate to unit 2 when most of the crystals analyzed correlate to unit 1 for samples MS-11-4.1 and MS-15-34a. a: Solid shapes—individual crystals within a sample. Open shapes - anomalous crystals. X's average sample end member concentrations. b: Error bars are associated with sample standard deviations and single crystal error bars represent standard deviation where there were two analysis points per crystal 


\section{LIST OF ABBREVIATIONS}

Ab. Albite — sodium rich feldspar end member

An. Anorthite - calcium rich feldspar end member

BPM. Blue Mountain Province

CRBG. Columbia River Basalt Group

DEM. Digital Elevation Model

DIT. Dinner Creek Tuff

DITEC. Dinner Creek Tuff Eruptive Center

DRE. Dense rock equivalent

EMP. Electron microprobe

GPS. Global Positioning System

HLP. High Lava Plains

HRCC. High Rock Caldera Complex

LAI. Low-aspect ratio ignimbrites

LIP. Large Igneous Province

LOVF. Lake Owyhee Volcanic Field

OIG. Oregon-Idaho Graben

Or. orthoclase — potassium rich feldspar end member

USGS. United States Geological Survey 


\section{INTRODUCTION}

During mid-Miocene bimodal volcanism in eastern Oregon, southwest Idaho and northwest Nevada, flood basalts erupted in addition to voluminous rhyolitic ashflow tuffs and lava flows, likely due to the Yellowstone hotspot (e.g. Lipman et al., 1972; Smith and Luedke, 1984; Hart and Carlson, 1987; Pierce and Morgan, 1992; Hooper et al., 2002; Shoemaker and Hart, 2002; Jordan et al., 2004; Humphreys and Coblentz, 2007; Wolff et al., 2008; Smith et al., 2009; Reidel et al., 2013, Camp, 2013; Ford et al., 2013). Voluminous flood basalts of the Columbia River Basalt Group (CRBG) dominated the initial phase of the Yellowstone hotspot eruptions, with the eruption of the Steens Basalt at 16.6 Ma (Figure 1) (e.g. Hooper et al., 2002; Coble and Mahood, 2012; Nash and Perkins, 2012; Barry et al., 2013; Mahood and Benson 2017).

Associated silicic volcanism includes a series of voluminous rhyolites erupted coeval to the CRBG that progressed in age as the Yellowstone hotspot evolved toward its current location (Figure 1) (Coble and Mahood, 2012; Nash and Perkins, 2012; Streck and Ferns, 2012; Streck et al., 2015). Approximately 90\% of the total volume of eastern Oregon and northern Nevada rhyolites erupted from three volcanic fields: Lake Owyhee, McDermitt and the High Rock Caldera Complex (HHRC) (Figure 1) (Rytuba and Vander Meulen, 1991; Cummings et al., 2000; Coble and Mahood, 2012). Lake Owyhee, McDermitt and the HHRC are situated near the edge of the Steens Basalts along a NNE trend close to the projection of the initial $\mathrm{Sr}\left({ }^{87} \mathrm{Sr} /{ }^{86} \mathrm{Sr}_{\mathrm{i}}\right)=$ 0.704 isopleth considered to be the boundary between the transitional continental crust and the accreted oceanic terranes (Armstrong et al., 1977; Farmer and DePaolo, 1983; 
Fleck and Criss 1985; Criss and Fleck 1987; Kistler and Lee 1989; Elison et al., 1990;

Leeman et al., 1992; Manduca et al., 1992; Wooden et al., 1999; Evans et al., 2002;

Fleck and Criss, 2007; Unruh et al., 2008; Gaschnig et al., 2011).
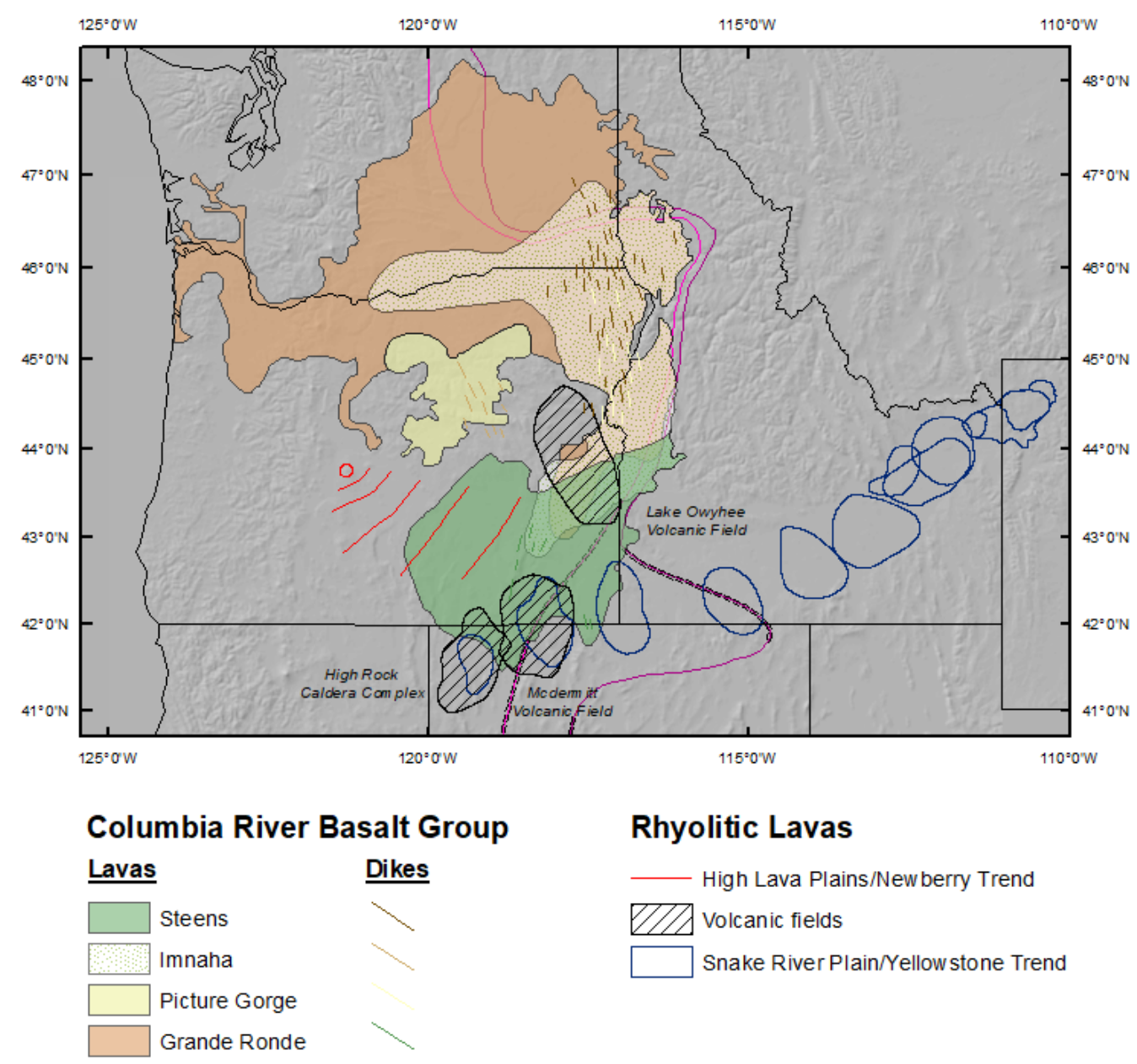

Rhyolitic Lavas

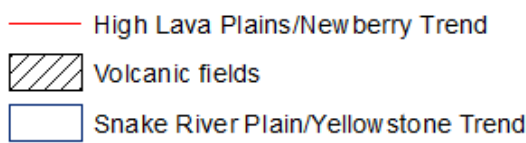

Figure 1. Regional overview modified from Benson and Mahood (2016). Columbia River Basalt Group member extents are after Reidel et al. (2013) and the generalized locations of feeder dikes after Tolan et al. (1989) and Camp et al. (2003). Red circle and lines delineate age progression of rhyolites in the High Lava Plains (HLP)/Newberry Trend from 0 to $10 \mathrm{Ma}$ (left to right). Coeval silicic volcanism that occurred within the McDermitt, and Lake Owyhee Volcanic Fields and the High Rock Calder Complex, approximate locations shown as black lined fields. Blue outlines indicate approximate locations of 16.4 to $0.6 \mathrm{Ma}$ (left to right) silicic centers associated with the Snake River PlainYellowstone trend after Smith et al. (2009). Purple line- $0.706{ }^{87} \mathrm{Sr}^{86} \mathrm{Sr}_{\mathrm{i}}$ isopleths and pink line$0.706{ }^{87} \mathrm{Sr} /{ }^{86} \mathrm{Sr}_{\mathrm{i}}$ isopleths are drawn using data from Armstrong et al. (1977), Farmer and DePaolo (1983), Fleck and Criss (1985), Criss and Fleck (1987), Kistler and Lee (1989), Elison et al. (1990), Leeman et al. (1992), Manduca et al. (1992), Wooden et al. (1999), Evans et al. (2002), Fleck and Criss (2007), Unruh et al. (2008), Pierce and Morgan, (2009) and Gaschnig et al. (2011). The black dashed outline on the 0.706 is where the line is inferred and on the 0.704 isopleth, indicates where it is poorly constrained due to Cenozoic volcanic cover. 
The Dinner Creek Tuff (DIT), situated at the northern end of the Lake Owyhee Volcanic Field (LOVF), was originally mapped as discontinuous exposures of ashflow tuff as early as 1937 by Gilully. The first mapped rhyolitic tuff separated older and younger mafic lava flows (Pardee and Hewett, 1941). Kittleman et al. (1965) initially named the Dinner Creek Welded Ash-flow Tuff type section after the marker bed referred to in the road log of geology along U.S. Highway 20 from Vale to Burns by Dole and Corcoran (1954). Additional mapping in area by Gray (1956) and Bowen (1956) referred to the DIT as the welded tuff in the Owyhee Basalt. The first methodical study of the DIT included descriptions of textural, mineralogical and chemical characteristics with an estimated extent of $\sim 2000 \mathrm{~km}^{2}$ centered along the Malheur River Gorge by Haddock (1967) (Figure 2).

Streck et al. (2015) utilized extents, lithologic variations, geochemical compositions and eruptive ages to correlate eastern Oregon ignimbrites and ash-fall tuff deposits to a newly redefined DIT comprised of four geochemically distinct cooling units that erupted from a common source area referred to as the Dinner Creek Eruptive Center (DITEC). They also correlated outcrops previously mapped as generic Miocene welded tuff as well as local units such as the "Mascall" or "Pleasant Valley" tuff of eastern Oregon are now correlated to one of the cooling units, increasing the known extent to $\sim 25,000 \mathrm{~km}^{2}$ (Figure 2). 

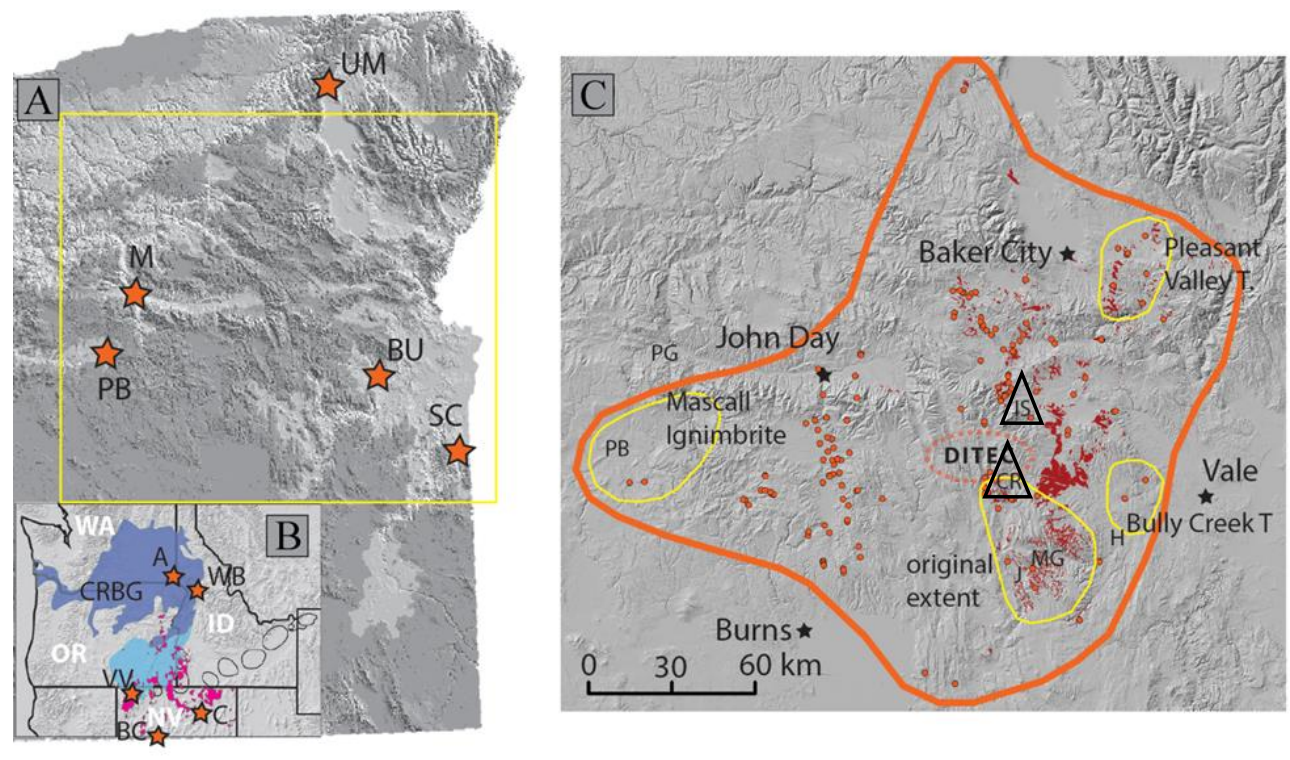

Figure 2. A and B: Regional overview, extent of the Dinner Creek Tuff and locations of correlative fallout units from Streck et al. (2015). A: Yellow frame is areal coverage of C. Stars indicate fallout localities: M-Mascall Formation, PB-Paulina Basin, BU—Bully Creek Formation, SC—Succor Creek (after Nash and Perkins, 2012), UM-Umatilla (after Ferns, personal data). B: Stars indicate regional fallout localities after Nash and Perkins (2012): NV—Nevada, BC—Buffalo Canyon, C Carlin, VV-Virgin Valley, ID - Idaho, WB-White Bird; WA-Washington; A-Asotin. Dark and light blue - CRBG after Camp and Ross, (2004); red — extent of 17-12 Ma rhyolites after Christiansen et al. (2002). C: Orange dots - investigated Dinner Creek Tuff locations; red-database of Oregon Department of Geology and Mineral Industries showing original Dinner Creek Tuff and unnamed tuffs correlated with Dinner Creek Tuff; thick orange line - envelope around most distal outcrops; dashed orange oval—likely source area of Dinner Creek Tuff eruptive center (DITEC); yellow—original extent of Dinner Creek Welded Tuff and previous generalized extents of local ignimbrites here correlated with Dinner Creek Tuff; Abbreviations: MG- Malheur Gorge, CR - Castle Rock, H-town of Harper, IS - Ironside Mountains, J-town of Juntura, PG-Picture Gorge, PB-Paulina Basin; T-tuff. The black triangle outlines represent the approximate locations of Castle Rock (CR) and Ironside Mountain (IS), the confirmed eruption sites for units 1 and 2, respectively.

Haddock (1967) and Wood (1979) hypothesized the DIT erupted from a single vent located in an area Rytuba and Vander Meulen (1991) considered to be part of the Castle Rock caldera. New mapping by Cruz (2017) partially confirms previous theories pertaining to the Castle Rock caldera which erupted unit 1 (Figure 2). Cruz (2017) also identified the new Ironside Mountain caldera as the source for unit 2 while precise source areas for unit 3 and 4 are still unknown (Figure 2). 
Based on field work alone, tracing out the extent of the individual cooling units that comprise the DIT outcrop exposure area is difficult (Streck et al., 2015). Neogene faults with more than $1,000 \mathrm{~m}$ of cumulative vertical displacement severely break up the discontinuous outcrops with younger volcanic and other sediments covering much of the outcrop areas and in most circumstances only one cooling unit is exposed (Ferns et al., 2010). Observed outcrop thickness for the DIT range from 3 - $70 \mathrm{~m}$ thick, but 3 - $8 \mathrm{~m}$ is the typical range (Kittlemen, et al., 1965; Haddock, 1967; Davenport, 1970; Streck et al., 2015).

The findings of this study establish minimal areal extent of the individual cooling units while updating the overall known areal extent of the Dinner Creek Tuff utilizing sample lithology, bulk rock and feldspar geochemistry. In addition, minimal erupted tuff volumes and dense rock equivalents (DRE) are calculated. The results of this study aide in the understanding of the lesser studied silicic portion of bimodal volcanism associated with the Yellowstone hotspot emplacement in eastern Oregon, northern Nevada and eastern Idaho. 


\section{BACKGROUND}

\section{Geologic Setting}

\section{Blue Mountain Province and Accreted Terranes}

Located in central and eastern Oregon, the Blue Mountains Province (BMP) is a middle Jurassic to late Jurassic ( 159 - $154 \mathrm{Ma})$ amalgamation of deformed islandarcs and related sedimentary terranes that accreted onto the North American craton 141 - 124 Ma ago during a period of arc-arc collision events (Getty et al., 1993; Selverstone et al., 1992; Dorsey and LaMaskin, 2007, 2008; LaMaskin, 2009; Schwartz et al., 2010; Schwartz et al., 2011; Anderson, 2013). Prior to accretion, volcanic island arcs, like modern day Japan and Indonesia made up extensive areas of the western United States, including California, Nevada, Oregon and Washington (LaMaskin, 2009). It is unclear whether the BMP formed from a single island arc situated well off shore or multiple island arcs, both near and off shore (Vallier, 1995; Dickenson, 1979; Ave Lallemant, 1995; Dorsey and LaMaskin, 2007; LaMaskin et al., 2008, LaMaskin, 2009). Accretion age estimates vary widely and introduce uncertainties to tectonic and geochemical models for North American crustal growth (Lund and Snee, 1988; Selverstone et al., 1992; Gray and Oldow, 2005; Snee et al., 2007; Dorsey and LaMaskin, 2007; LaMaskin et al., 2008; LaMaskin, 2009). The units that comprise the BMP include: Wallowa Terrane, Baker Terrane, Olds Ferry Terrane and Izee Terrane (Figure 3). 


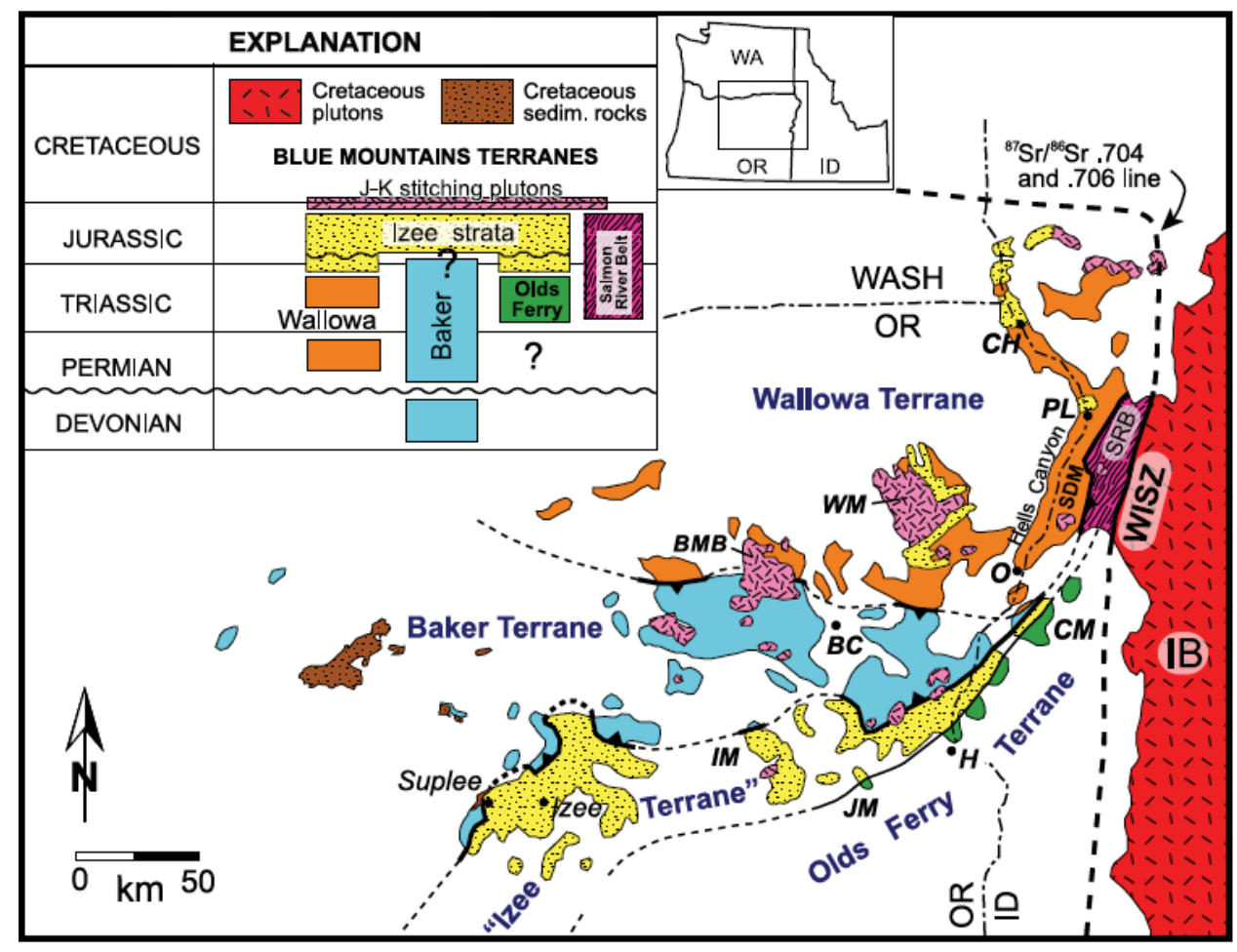

Figure 3. Simplified geologic of the Blue Mountains Province showing pre-Cenozoic geology, from Dorsey and LaMaskin (2008). White areas represent Cenozoic rocks and deposits. BC, Baker City; BMB, Bald Mountain batholith; H, Huntington; IB, Idaho Batholith; IM, Ironside Mountain; JM, Juniper Mountain; O, Oxbow; PL, Pittsburg Landing; CM, Cuddy Mountains; CH, Coon Hollow; SDM, Seven Devils Mountains; SRB, Salmon River belt; WM, Wallowa Mountains and Wallowa batholith; WISZ, Western Idaho Shear Zone.

\section{Wallowa Terrane}

The northernmost terrane in the BMP is the Wallowa terrane. The Permian to early Jurassic rocks that comprise the Wallowa terrane record the evolution of an intraoceanic island arc system and change to clastic input from the Baker terrane (Vallier, 1977, 1995; Follo, 1986, 1992, 1994; LaMaskin et al., 2009; Anderson, 2013). Rock types associated with the Wallowa terrane include: volcaniclastic and volcanic rocks, primarily from the early Permian to late Triassic, Triassic and Jurassic aged sedimentary rocks and plutonic rocks from the Jurassic to the early Cretaceous 
(Avé Lallemant, 1995; Vallier, 1995; Anderson, 2013). Magmatism associated with the Wallowa terrane consists of two main pulses. The initial pulse of silicic arc magmatism from 265.4-248.8 Ma, followed by deformation and erosion of the Wallowa arc and a mafic to intermediate pulse between $~ 229.4-229.1$ based on U-Pb zircon dating of the Cougar Creek Complex (Kurz et al., 2012). Occasional intermittent volcanism occurred until the end of the Middle Jurassic ( 161 Ma) (Avé

Lallemant, 1995; Vallier et al., 1977; Follo, 1994; White and Vallier, 1994; Anderson, 2013). While the terrane reached amphibolite-facies conditions in some localities, such as the Cougar Creek Complex, it exhibits regionally metamorphosed to greenschist facies conditions throughout (Vallier, 1995).

\section{Olds Ferry Terrane}

The Olds Ferry Terrane is comprised of middle Triassic to Lower Jurassic plutonic, volcanic, epiclastic and marine volcaniclastic rocks that formed in an islandarc setting that corelate to the Quesnel terrane in British Columbia, the Cordilleran fringing-arc system in western Nevada (Black Rock terrane) and eastern California (Brooks and Vallier 1978; Brooks 1979; Oldow et al., 1989; Vallier 1995; Wyld and Wright, 2001; Gray and Oldow, 2005; Dorsey and LaMaskin, 2007, 2008; Tumpane et al., 2008; LaMaskin, 2009). The island arc is lithologically like the Wallowa terrane with volcanic rocks from the late Triassic to early Jurassic and plutonic rocks mainly from the middle Triassic to late Triassic (Iron Mountain and Brownlee pluton), but dissimilar to the Wallowa terrane with poor exposure, the absence of a well-defined age and failure to exceed greenschist facies conditions (Brooks and Vallier, 1978; 
Tumpane, 2010; Anderson, 2013). Dorsey and LaMaskin consider the Huntington and Weatherby Formations to be part of the Olds Ferry terrane and are comprised of clastic sedimentary rock, limestone and keratophyre rocks, generally defined as a felsic tuff or flow characterized by phenocrysts of quartz and albite as well as a high Na to K ratio (Anderson, 2013).

\section{Baker Terrane}

Situated between the Wallowa and Olds Ferry terranes, the Baker terrane is an accretionary complex comprised of three sub terranes: Bourne, Greenhorn and Burnt River Schists (Anderson, 2013). Early Jurassic Elkhorn Ridge Argillite, consisting mainly of disrupted chert and argillite with lesser amounts of tuffaceous argillite, limestone and conglomerate dominates the Bourne sub terrane (Schwartz et al., 2010; Schwartz et al., 2011b). The Greenhorn sub terrane is a serpentinite-matrix mélange enclosing large blocks of chert-argillite breccias, volcaniclastic breccias, keratophyre, meta-plutonic rocks with some amphibolitic rocks (Schwartz et al., 2011b). The Bourne subterrane is consistently lower greenschist facies in metamorphism while the Greenhorn sub terrane ranges from upper greenschist facies to lower amphibolitefacies (Schwartz et al., 2010). The Burnt River Schist is a heterogeneous sub terrane dominated by siliceous phyllite and slatey argillite that contains marble, metaplutonic rocks (ranging from felsic to mafic) and keratophyre rocks (Anderson, 2013). Metamorphism is greenschist facies, but a higher greenschist facies than the 
Greenhorn sub terrane, and the contact metamorphism near the plutons reaches hornblende to hornblende-pyroxene hornfels facies (Ashley, 1995).

Izee Terrane

The Izee terrane consists mainly of Triassic to Jurassic aged clastic sedimentary rocks primarily exposed in the southern portion of the BMP (Dickinson, 1979; Avé Lallemant, 1995). Izee terrane sedimentary and rare volcanic rocks correlate with limestone and volcanic rocks from the Huntington and Weatherby Formations associated with the Olds Ferry terrane (Dickinson, 1979; Avé Lallemant, 1995; Dorsey and LaMaskin, 2007). Furthermore, work by Dorsey and LaMaskin (2007) correlated Jurassic age sediments with sediments from the Coon Hollow formation associated with the Wallowa terrane and suggest that the Izee terrane is not an actual terrane but a regional overlap assemblage of largely Jurassic sedimentary rocks which unconformably overlies the Baker and Olds Ferry terranes. The Izee terrane rarely exhibits metamorphism no higher than the greenschist facies (Dorsey and LaMaskin, 2007).

\section{Columbia River Basalt Group}

Large igneous provinces (LIP) are high volume $\left(>100,000 \mathrm{~km}^{3}\right)$ intraplate magmatic events comprised largely of flood basalts, dyke swarms and their associated plumbing, sill complexes, mafic-ultramafic layered intrusions and in some cases, silicic igneous rocks (e.g. Coffin and Eldholm 1994, 2001, 2005; Ernst and Buchan, 2001; Courtillot and Renne 2003; Ernst et al. 2005; Bryan and Ernst 2008). 
Associated with the initial phase of the Yellowstone hotspot mantle plume, the Columbia River Basalt Group (CRBG) is the smallest (over 210,000 $\mathrm{km}^{2}$ with an estimated basalt volume of $\sim 210,000 \mathrm{~km}^{3}$ ) and youngest $(16.7 \mathrm{Ma}-5.5 \mathrm{Ma}) \mathrm{LIP}$ in the world (e.g. Nash and Perkins, 2012; Barry et al. 2013; Reidel et al., 2013). Excellent preservation and a well-defined stratigraphy exposed across the Pacific Northwest make the CRBG a commonly cited model for study of LIPs worldwide (Reidel et al., 2013) (Figure 1).

Basaltic flood lavas originated in a back arc setting between the Cascade volcanic arc and the Rocky Mountains (Reidel et al., 2013). The southern Oregon Plateau near the northern limit of Basin and Range extension is considered the initial eruption site, but eruptions sites swiftly migrated north creating a series of generally north-northwest-trending linear fissure systems (Reidel et al., 2013). Lava flows inundated the Columbia River Basin before heading westward through the Cascade Range, Willamette Valley and Coast Range before flowing into the offshore Miocene fore arc basins (Reidel et al., 2013).

While the CRBG erupted over a period of $\sim 11$ million years, $\sim 93 \%$ erupted with a $\sim 1.1$ million-year period from 16.7 - 15.6 Ma (Reidel et al., 2013). This main eruptive phase produced the Steens, Imnaha, Grande Ronde, Prineville and Picture Gorge Basalts (Figure 4) (Reidel et al., 2013). The Grande Ronde eruptive period marks the height of the CRBG flood basalts with $\sim 72 \%$ of the volume being erupted over 400,000 years from 16 - 15.6 Ma (Barry et al., 2013; Reidel et al., 2013). Many of the flows that erupted during the main phase and into Wanapum time exceeded $1,000 \mathrm{~km}^{3}$ and extend hundreds of kilometers from their eruption sites (Tolan et al., 
1989; Reidel et al., 1989a; Reidel, 1998, 2005; Reidel and Tolan, 2013). In total, the CRBG consists of seven main formations that include those of the main phase as well as the Wanapum Basalt and Saddle Mountains Basalt in addition to various formal and informal members (Reidel et al., 2013).

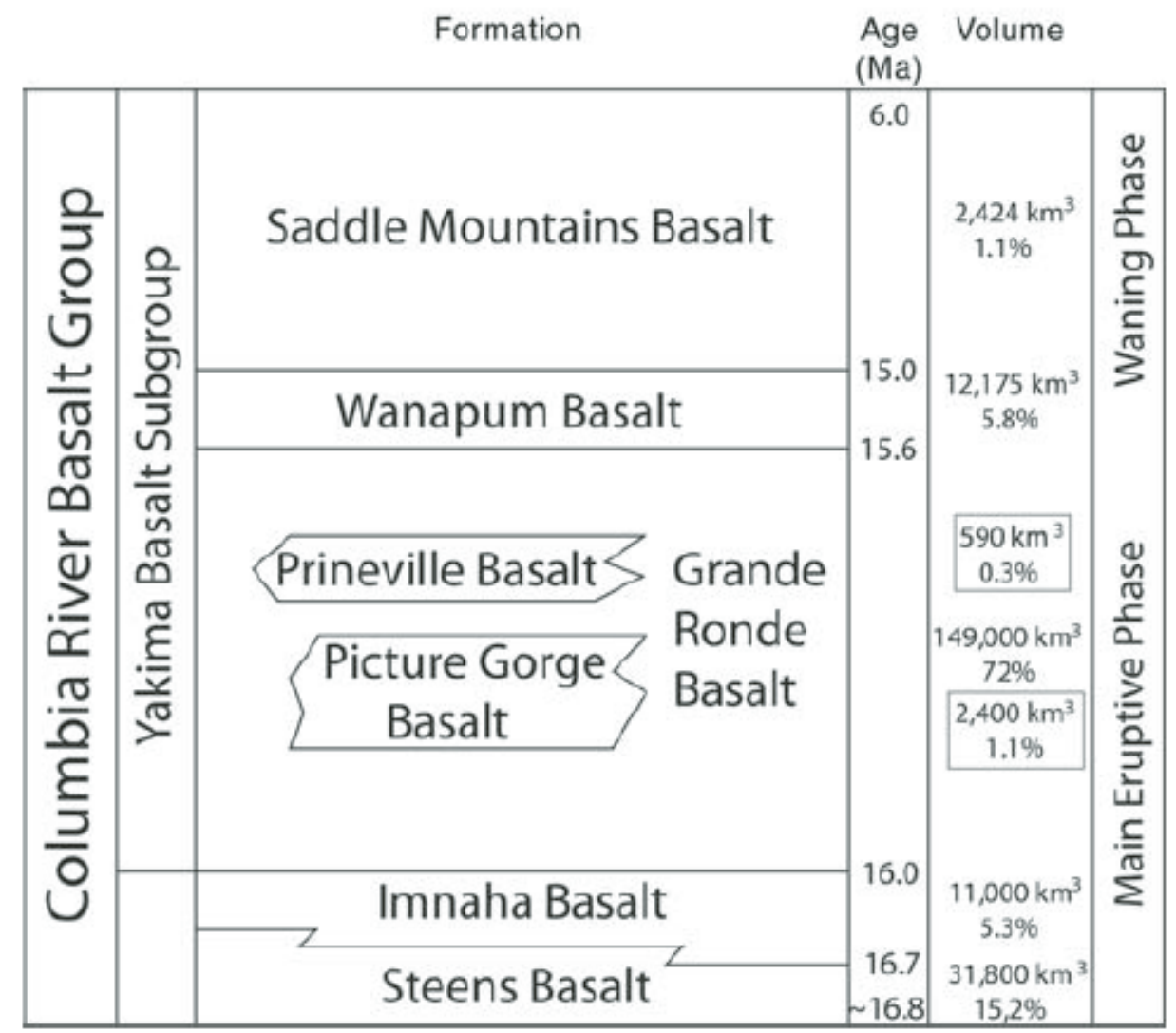

Figure 4. Major subdivisions of the Columbia River Basalt Group from Reidel et al. (2013).

\section{Rhyolitic volcanism}

Erupting coeval to the main phase of the CRBG, mid-Miocene silicic volcanism erupted a minimum of $\sim 4,000 \mathrm{~km}^{3}$ of rhyolites from eruptive centers scattered over $\sim 25,000 \mathrm{~km}^{2}$ across eastern Oregon, northern Nevada and southeastern Idaho between 16.5 and 15.0 Ma (Christiansen and Yeats, 1992; Pierce and Morgan, 
1992; Brueseke et al., 2008; Shervais and Hanan, 2008; Coble and Mahood, 2012;

Streck et al., 2015; Benson and Mahood, 2016). While the eastward-younging silicic volcanism associated with the initial phase of the Yellowstone hotspot and the subsequent passage of the North American plate over the plume tail represents a significant amount of silicic volcanism in the area, the majority of rhyolites erupted from one of three major caldera complexes: the Lake Owyhee Volcanic Field (LOVF), McDermitt Volcanic Field and High Rock Caldera Complex (HHRC)

(Figure 1) (Rytuba and McKee, 1984; Pierce and Morgan, 1992, 2009; Camp and Ross, 2004; Nash et al., 2006; Hooper et al., 2007; Leeman et al., 2008; Shervais and Hanan, 2008, Coble and Mahood, 2012; Benson and Mahood, 2016).

\section{Lake Owyhee Volcanic Field}

Rhyolitic volcanism in the northern section of the LOVF include distal air-fall tuffs and limited exposures of non-welded lithic tuff but are mainly comprised of large rhyolite ash-flow tuffs, rhyolitic lavas originating from eruptive centers and caldera complexes located near the center or in the southern part of the LOVF, most notable is the Dinner Creek Tuff associated with this study (Ferns et al., 2017). Also found in the northern part of the LOVF is the rhyolitic center at Dooley Mountain that is composed of $\sim 2,400 \mathrm{~m}$ thick low to high-silica rhyolites consisting of welded tuff, tuff breccia with 2 m diameter clasts, pyroclastic dikes and rhyolitic lava domes (Reef, 1983; Whitsun, 1988; Bailey, 1990; Evans, 1992; Ferns and McClaughry 2013; Large, 2016). The onset of the eruption period at the Dooley Rhyolite complex occurred between $15.59 \pm 0.04 \mathrm{Ma}$ and $16.15-16.00 \mathrm{Ma}$, after the emplacement of DIT cooling 
unit 1 and ended no later than $\sim 15.2 \pm 0.04$ Ma (Barry et al., 2013; Hess, 2014; Streck et al., 2015; Large, 2016).

In the southern portion of the LOVF, large rhyolite lava flows and caldera complexes extend over the northeastern Steens Basalt, east through the Malheur River gorge and into western Idaho, including the Littlefield Rhyolite (Kittleman et al., 1967; Webb et al., 2015, 2016; Ferns et al., 2017). Two geochemically distinct largevolume rhyolite lava flow packages that comprise the Littlefield Rhyolite known as the lower Littlefield Rhyolite (16.12 $\pm 0.04 \mathrm{Ma})$ and upper Littlefield Rhyolite (16.05 \pm 0.04 Ma) (Webb et al., 2015, 2016; Ferns et al., 2017). The Littlefield Rhyolite most likely originated from a series of north-south trending dikes along the western margin of Oregon-Idaho graben and extend over a 1,200 $\mathrm{km}^{2}$ area, overlying unit 1 of the DIT (Kittleman et al., 1967; Lees, 1994; Streck et al., 2015; Webb et al., 2015, 2016; Ferns et al., 2017). Theories suggest that Littlefield Rhyolite lava flows along the Malheur River are equivalent to the Cottonwood Mountain rhyolite described by Evans and Binger (1999) (Edwards, 2013; Webb et al., 2015, 2016). A caldera complex at Mahogany Mountain erupted both the Spring Creek Tuff (15.4 Ma) and the Leslie Gulch Ash-Flow Tuff Member of the Sucker Creek Formation (15.5 Ma) (Kittleman et al., 1967; Rytuba and others, 1985; Vander Meulen et al., 1987a, b; Rytuba and Vander Meulen, 1991; Marcy et al., 2013).

\section{McDermitt Volcanic Field}

The McDermitt Volcanic Field is comprised of basaltic to rhyolitic lavas, calderas and outflow sheets of associated rhyolitic ignimbrites (Benson and Mahood, 
2017). The $0.704{ }^{87} \mathrm{Sr} /{ }^{86} \mathrm{Sr}_{\mathrm{i}}$ isopleth extends through the complex, but the exact location is poorly constrained due to Cenozoic volcanic cover (Benson and Mahood 2016, 2017). Rytuba and McKee (1984) originally identified seven regionally extensive tuffs erupting from seven different calderas, but recent work by Benson and Mahood (2017) utilized new ${ }^{40} \mathrm{Ar} /{ }^{39} \mathrm{Ar}$ ages, the stratigraphic relations of ignimbrites, and geochemical correlations of outflow sheets to refine the stratigraphy to include only four significant ignimbrites: the Tuff of Oregon Canyon, zoned from high-silica alkali rhyolite to trachyte $(16.468 \pm 0.006 \mathrm{Ma})$, the Tuff of Trout Creek Mountains, zoned from high-silica alkali rhyolite to low-silica alkali rhyolite $(16.415 \pm 0.007 \mathrm{Ma})$, the Tuff of Long Ridge, zoned from high-silica alkali rhyolite to low-silica alkali rhyolite (16.328 $\pm 0.013 \mathrm{Ma})$ and the Tuff of Whitehorse Creek, a low-silica alkali rhyolite $(15.556 \pm 0.014 \mathrm{Ma})$.

\section{High Rock Caldera Complex}

The McDermitt volcanic field was frequently cited as the starting point of the Snake River/Yellowstone trend, but work by Coble and Mahood (2012) discovered that the HRCC contains the initial silicic center associated with the Yellowstone hotspot (Pierce and Morgan, 1992, 2009; Camp and Ross, 2004; Nash et al., 2006; Hooper et al., 2007; Leeman et al., 2008; Shervais and Hanan, 2008). In addition, the HHRC is comprised of four individual calderas and their associated eruptive products that decrease in age from northeast to southwest (Coble and Mahood, 2016). The Virgin Valley caldera is the oldest caldera (16.38 Ma) and largest (minimum of $\sim 52$ $\mathrm{km}^{3} \mathrm{DRE}$ ) and erupted the chemically zoned alkali rhyolite Idaho Canyon Tuff. The 
16.34 Ma old Badger Mountain Caldara erupted the strongly porphyritic, low-silica rhyolite Summit Lake Tuff that overlaps the southern margin of the Virgin Valley caldera (Coble and Mahood, 2016). Approximately $55 \mathrm{~km}^{3}$ DRE of the crystal-poor alkali rhyolite Antelope lavas and compositionally comparable small-volume pyroclastic units denoted as the tuff of Big Mountain that vented along reactivated ring faults of the Virgin Valley caldera erupted $\sim 100,000$ years after the Summit Lake Tuff (Coble and Mahood, 2016). Curvilinear faults and vent alignments for 16.3 - 16.2 Ma crystal-poor alkali rhyolite lavas are found south of the Badger Mountain caldera adjacent to densely welded ignimbrites of comparable composition recently named tuffs of Coyote Spring (16.27 Ma) and Trough Mountain (16.25 - 16.15 Ma). The Hanging Rock caldera (16 Ma) is comprised of $\sim 65 \mathrm{~km}^{3}$ DRE of strongly porphyritic, alkali rhyolite known as the Soldier Meadow Tuff (Coble and Mahood, 2016). Petrographically and geochemically similar alkali rhyolite lavas like the Soldier Meadow Tuff erupted within the caldera shortly after collapse. The youngest (15.70 Ma) and southernmost caldera, the Cottonwood Creek caldera formed during the eruption of the strongly zoned high-silica alkali rhyolitic tuff of Yellow Rock Canyon ( $220 \mathrm{~km}^{3}$ DRE pre-caldera and post-caldera ring-fracture alkali rhyolite lava volume and $\sim 50 \mathrm{~km}^{3}$ DRE outflow ignimbrite minimum volume) (Coble and Mahood, 2016).

\section{Snake River Plane/Yellowstone Hotspot trend}

The emplacement of the Yellowstone hotspot began when the ascending spheroidal plume head $\sim 300 \mathrm{~km}$ in diameter reached the base of the North American plate, flattening outward into a disk $\sim 1000 \mathrm{~km}$ in diameter $\sim 17 \mathrm{Ma}$. The initial 
emplacement initiated the CRBG, the intrusion of a large basaltic dike system and rhyolitic volcanism (Pierce and Morgan, 1990, 1992, 2009; Draper, 1991; Parsons et al., 1994; Camp, 1995; Parsons, 1995; Takahahshi et al., 1998; Thompson, 1998; John et al., 2000; Glen and Ponce, 2002; Pierce et al., 2002; Camp and Ross, 2004; Jordan et al., 2004; Rowley, 2001). Rhyolitic volcanism associated with the Yellowstone hotspot trend is thought to have initiated $\sim 16.4 \mathrm{Ma}$ in the HRCC and extends northeast to modern day Wyoming (Figures 1 and 5).

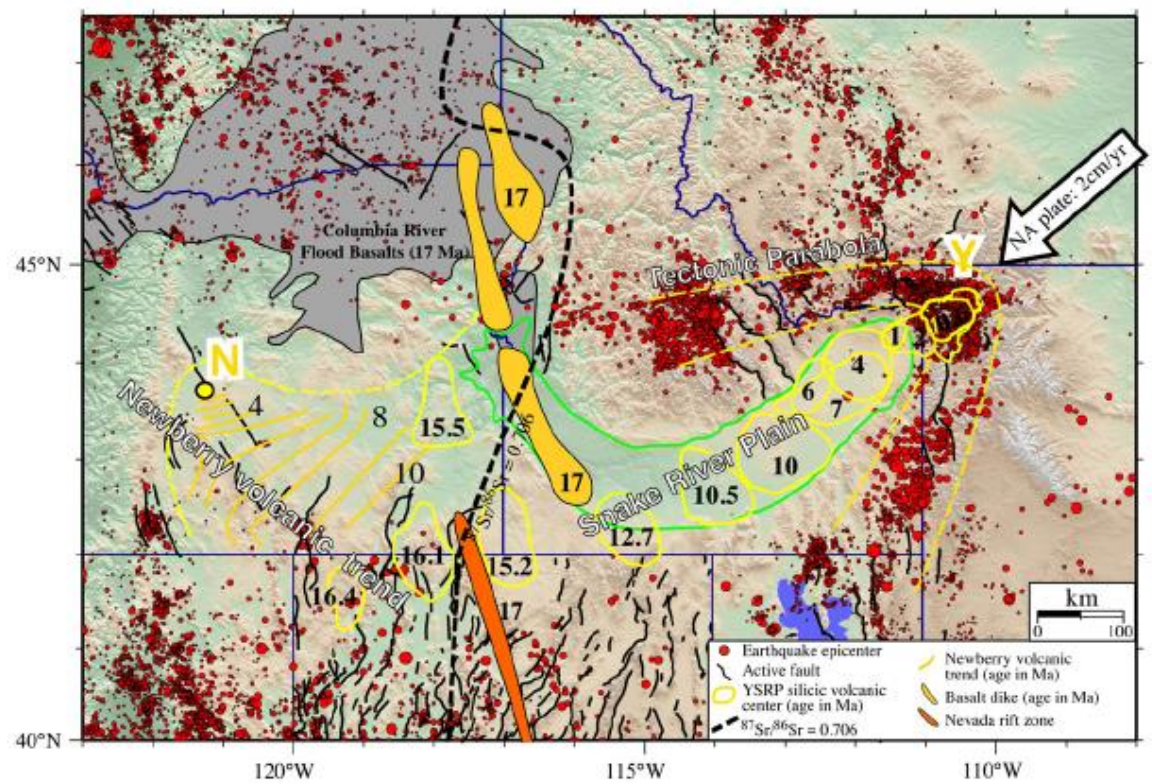

Figure 5. Track of the Yellowstone hotspot (Y) from Smith et al. (2009) indicating the relative motion of age-transgressive ESRP silicic volcanic centers (Armstrong et al., 1975; Smith and Braile, 1984; Pierce and Morgan, 1990, 1992; Kuntz et al., 1992; Smith and Braile, 1994; Perkins and Nash, 2002) opposite to the direction of North America plate motion (large arrow) (Smith and Sbar, 1974). Green outline is the topographically low area occupied by the Snake River Plain. Yellow outlines are post-17 Ma silicic volcanic centers containing multiple caldera-forming eruptions (Christiansen, 1984, 2001). Columbia River flood basalts are gray (Perkins and Nash, 2002). Red dots are historic earthquake epicenters taken from compilations of the University of Utah of M1.5 - 7.5 earthquakes. Black lines show late Quaternary faults; Cenozoic basaltic dikes (age in Ma) are shown in yellow and orange (Zoback and Thompson, 1978; Camp, 1995; Glen and Ponce, 2002). ${ }^{87} \mathrm{Sr} /{ }^{86} \mathrm{Sr}$ isotope boundary for the 0.706 values is shown as a black-dashed line and is interpreted to separate continental lithosphere to the east and oceanic lithosphere to the west (Armstrong et al., 1977; Farmer and DePaolo, 1983). The tectonic parabola is defined by the bow shaped pattern of high topography and seismicity surrounding the YSRP (yellow dashed lines) (Smith et al., 1985; Smith and Arabasz, 1991; Anders and Sleep, 1992). The Newberry trend of silicic volcanism extends northwest across southeast Oregon to the Newberry caldera (N) (MacLeod et al., 1976). 


\section{Oregon-Idaho Graben}

The 50 - $60 \mathrm{~km}$ wide, $100 \mathrm{~km}$ long Oregon-Idaho graben evolved as part of a $\sim 1,100 \mathrm{~km}$ long mid Miocene synvolcanic rift system extending from southeastern Washington to northern Nevada that includes the CRBG dike swarms, the Baker and La Grande basins and the Nevada rift (Figure 6) (Hooper and Swanson, 1990; Zoback et al., 1994; Cummings et al.,1994, 2000). Situated in the southern portion of the LOVF, the north to northwest trending graben formed during a period linked to eastwest rifting, fading CRBG eruptions and widespread rhyolitic volcanism ( 15.5 to 10.5 Ma) (Cummings et al., 2000). After the initial subsidence, Cummings et al. (2000) describe the graben evolution through three stages. Stage 1 (15.3-14.3 Ma) features fluvial and lacustrine sediment deposition across the graben width. Stage 2 (14.3-12.6 Ma) coincides with synvolcanic collapse along intra-graben faults, subdivision of the graben into individual sub-basins by $15-20 \mathrm{~km}$ wide horsts and graben structures and intra-graben calc-alkalic magmatism. Sedimentation dominated stage 3 (12.6-10.5 Ma) with volcanic material, lacustrine and fluvial sediments filling the sub-basins across the width of the graben. By 10.5 Ma crustal extension migrated to the western Snake River Plain (Cummings et al., 2000). 


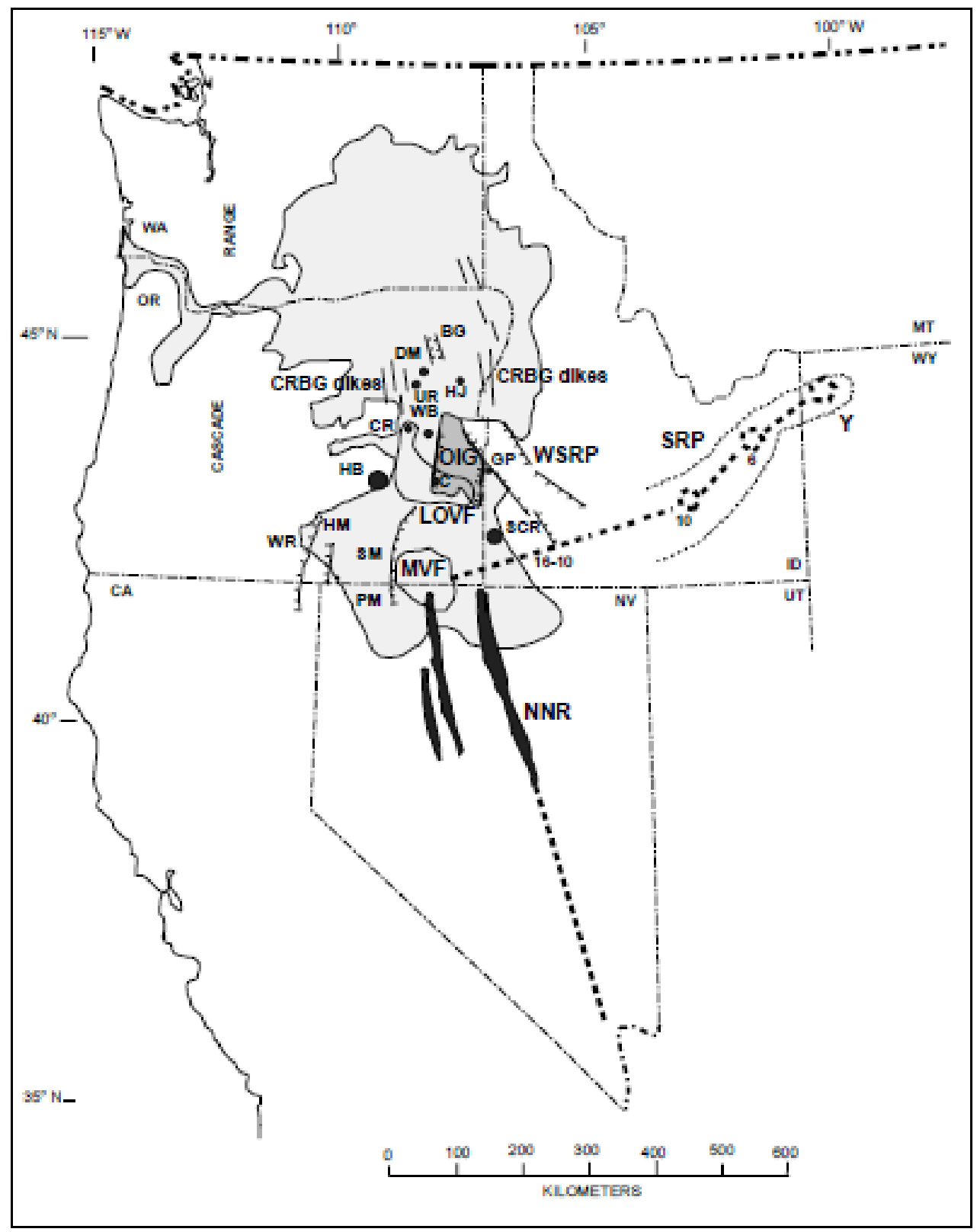

Figure 6. Regional map showing the Oregon-Idaho graben (OIG) in context of the dike swarms of the Columbia River Basalt Group (CRBG dikes), the northern Nevada rift (NNR), and the western Snake River Plain (WSRP) after Cummings et al. (2000). Regional features near the Oregon-Idaho graben depicted by additional symbols as follows: $\mathrm{BG}$ - Baker graben; $\mathrm{C}-$ Crowley; CR - Castle Rock; DM —Dooley Mountain; GP — Graveyard Point, HB — Harney basin; HJ—Huntington Junction; HM — Hart Mountain; LOVF—Lake Owyhee volcanic field; MVF-McDermitt volcanic field; PM-Pueblo Mountains; SM — Steens Mountain; SCR — Silver City Range; SRP—Snake River Plain; WB—Westfall Butte; WR — Warner Range; UR - Unity Reservoir; Y - Yellowstone Plateau. WA—Washington; OR — Oregon; ID—Idaho; MT —Montana; WY - Wyoming; UT_Utah; NV—Nevada; CACalifornia. Numbers displayed along the Snake River plain (SRP) show the age progression of rhyolitic volcanism. 


\section{Dinner Creek Tuff}

The DIT erupted during a period of rhyolitic volcanism coeval to the flood volcanism associated with the CRBG (Streck and Ferns, 2012; Streck et al., 2015) (Figure 7). Although DIT rhyolites have nearly identical compositions, Streck et al. (2015) discovered different ages, subtle geochemical and lithological differences that divide the DIT into four cooling units. Except for unit 4, the units are lithologically very similar (Figure 8). ${ }^{40} \mathrm{Ar} /{ }^{39} \mathrm{Ar}$ analysis indicates the unit ages: $16.15-16 \mathrm{Ma}$ (unit 1), 15.6-15.5 Ma (unit 2), 15.46 Ma (unit 3) and 15.0 Ma (unit 4) (Streck et al., 2015;

Streck, pers. comm., 2017).

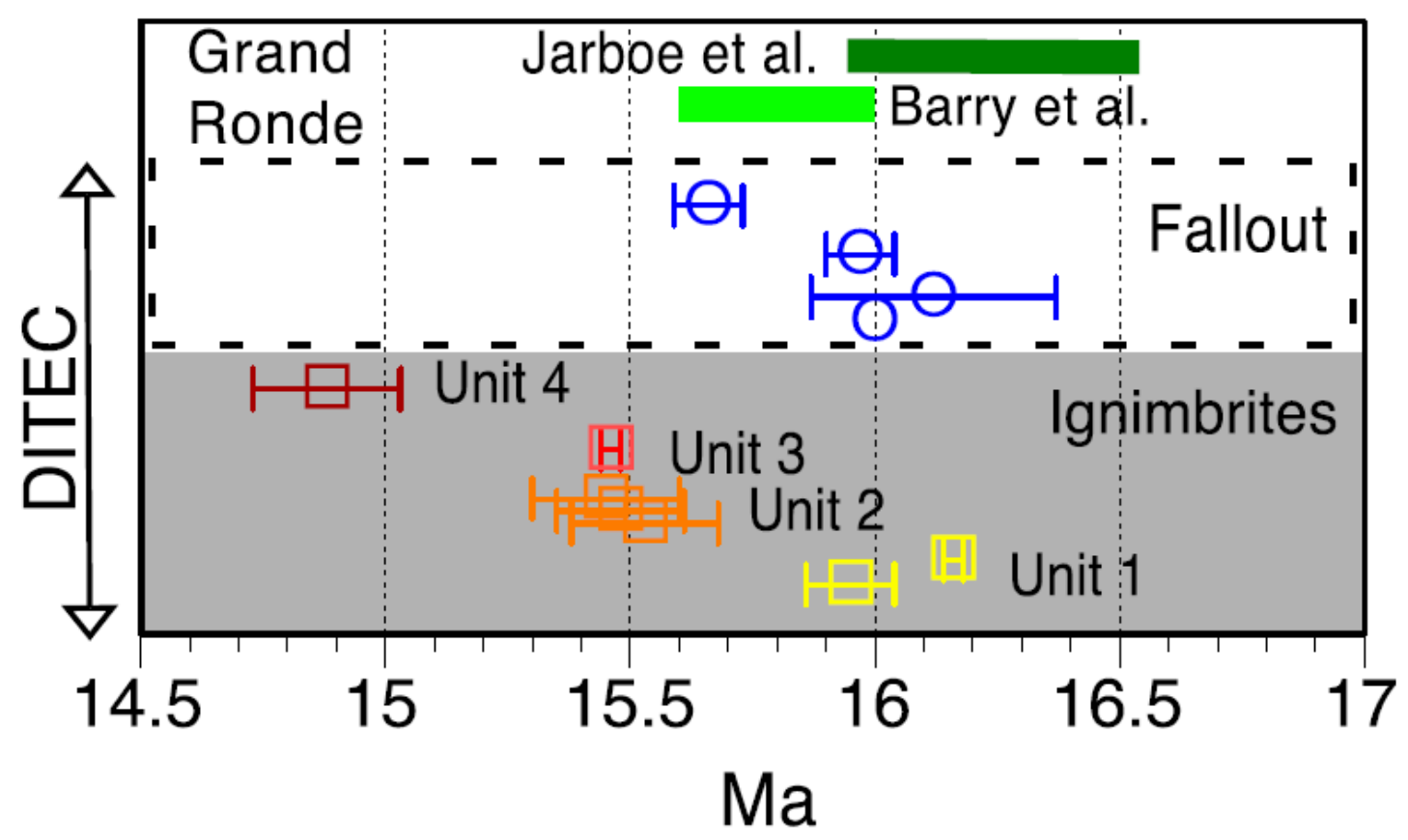

Figure 7. Age relationships of ignimbrite and fallout tuffs originating from the Dinner Creek Tuff eruptive center in relation to eruption period of Grande Ronde Basalt lavas based on the Barry et al. (2013) and Jarboe et al. (2010) chronology after Streck et al., (2015). The DITEC on the y axis highlights the rhyolites that erupted from the DITEC; proposed age ranges for the Grande Ronde member of the CRBG are shown above. 
DIT units range from welded, marked by a basal vitrophyre typically overlain by primarily devitrified zones, to only incipiently welded tuff sections throughout. All cooling units are crystal poor with phenocryst contents of $<1 \%-5 \%$ (Streck, et al., 2015). Outcrops range from pumice poor $(\sim 5 \%)$ to pumice rich $(\sim 30 \%)$; small pumice is typically $\sim 2-4 \mathrm{~cm}$ in the longest dimension and rarely exceeds $15 \mathrm{~cm}$. Pumice colors range from white to tan to dark gray, but in single outcrops only one or two colors are evident (Figure 8) (Streck, et al., 2015). Anorthoclase or Na-rich plagioclase feldspar dominate individual cooling unit mineralogy with subordinate amounts of titanomagnetite and extremely rare pyroxenes. Due to the crystal-poor nature of the units, exact accessory phase mineralogy is currently unknown (Streck et al., 2015).

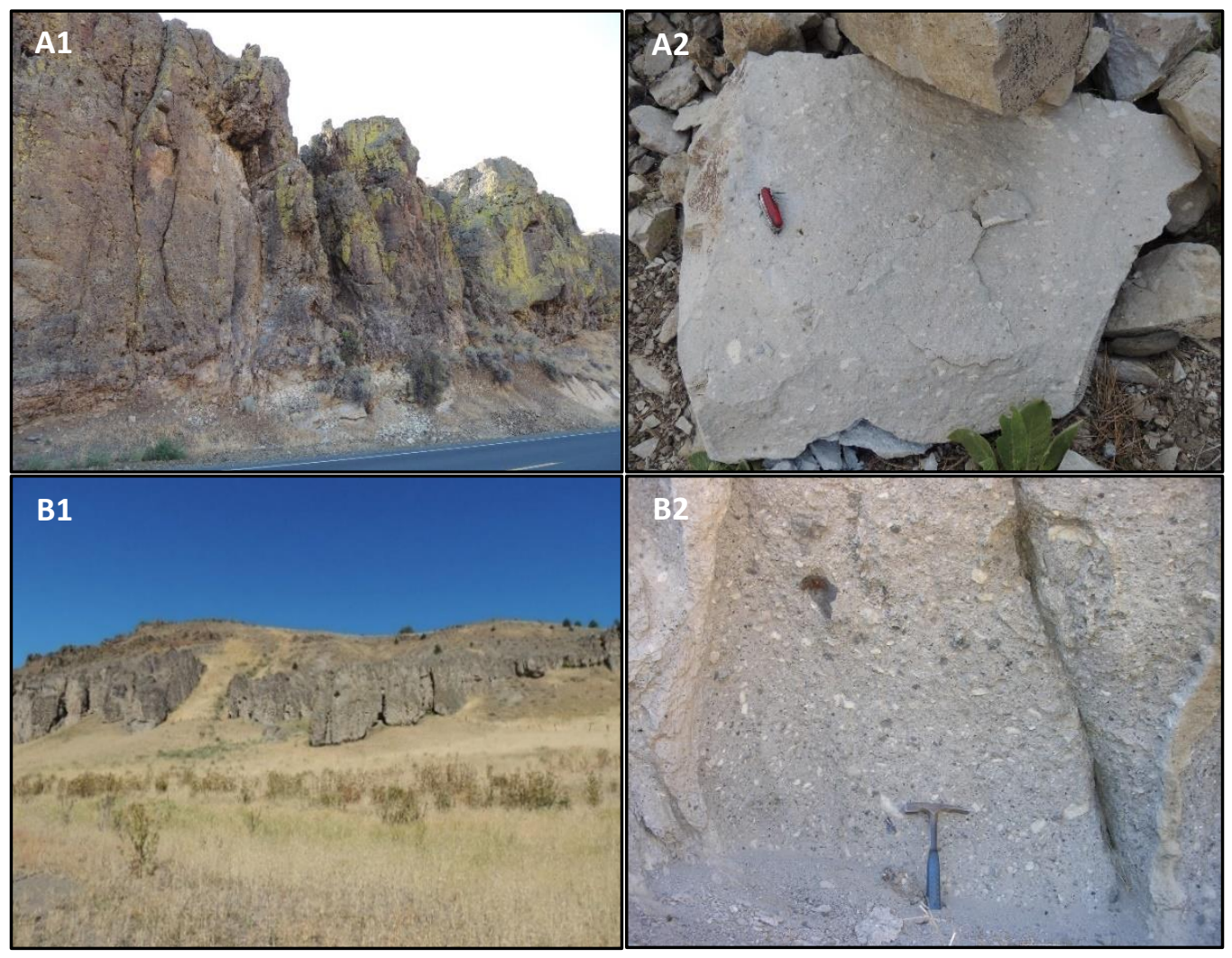




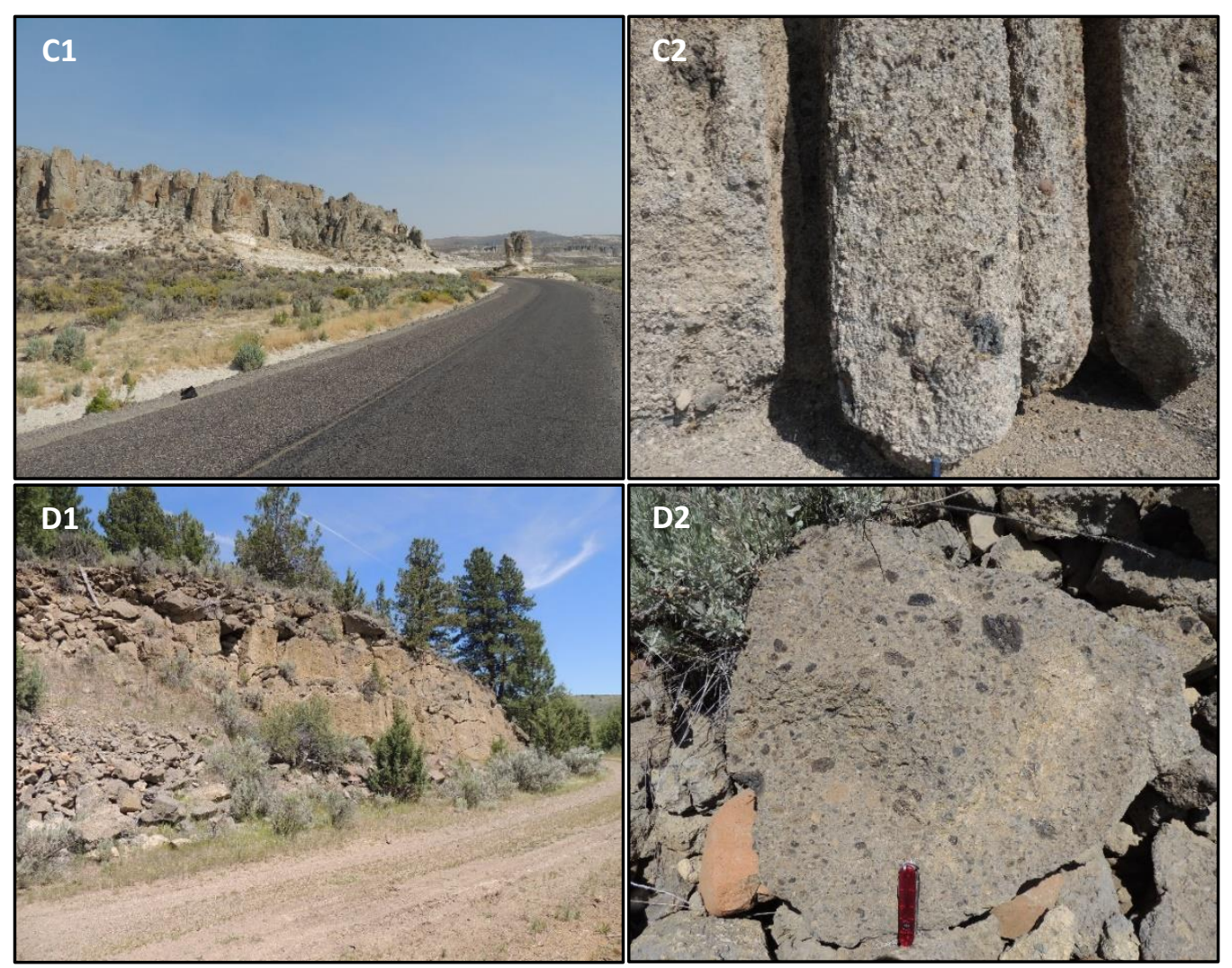

Figure 8. Dinner Creek Tuff unit photographs by Martin Streck. A1: Unit 1 type section in Malheur Gorge. A2: Unit 1 sample with white pumices. B1: Unit 2 outcrop east of John Day, OR. B2: Unit 2 near John Day, OR. C1: Unit 3 outcrop previously identified as the Bully Creek Tuff. C2: Unit 3, the recently correlated Bully Creek Tuff. D1: Unit 4 outcrop. D2: Unit 4 with vesicular globules of tholeiitic andesite glass that resemble Hunter Creek Basalt (Streck et al., 2015).

Bulk tuff compositions vary from a high-silica rhyolite (unit 1) to low-silica rhyolites (units 2 and 3) to dacitic (unit 4) (Figure 9) (Streck, et al., 2015). Unit 1 FeO* ${ }^{*} \mathrm{MgO}, \mathrm{TiO}_{2}$ and $\mathrm{Al}_{2} \mathrm{O}_{3}$ concentrations are lower than the other units (Streck, et al., 2015). Except for $\mathrm{Sr}(<60 \mathrm{ppm})$ unit 1 is enriched in trace elements concentrations (Streck, et al., 2015). Units 2 and 3 tend to be higher in $\mathrm{FeO}^{*}, \mathrm{MgO}, \mathrm{TiO}_{2}$ and $\mathrm{Al}_{2} \mathrm{O}_{3}$ and enriched in $\mathrm{Sr}, \mathrm{Ba}$ and $\mathrm{Ti}$ with unit 2 containing more $\mathrm{Sr}$ than unit 3. La, Ce, U and $\mathrm{Rb}$ concentrations are consistent with unit 1 . Unit 4 is depleted in most trace elements, but enriched in $\mathrm{Eu}$ and $\mathrm{Sr}$ (Streck et al., 2015). 
While bulk rock compositions distinguish unit 3 from unit 4 as well as from the other units, feldspar compositions for these units are indistinguishable. Unit 1 and unit 2 feldspar compositions differentiate them from one another as well as units 3/4 (Figure 10).
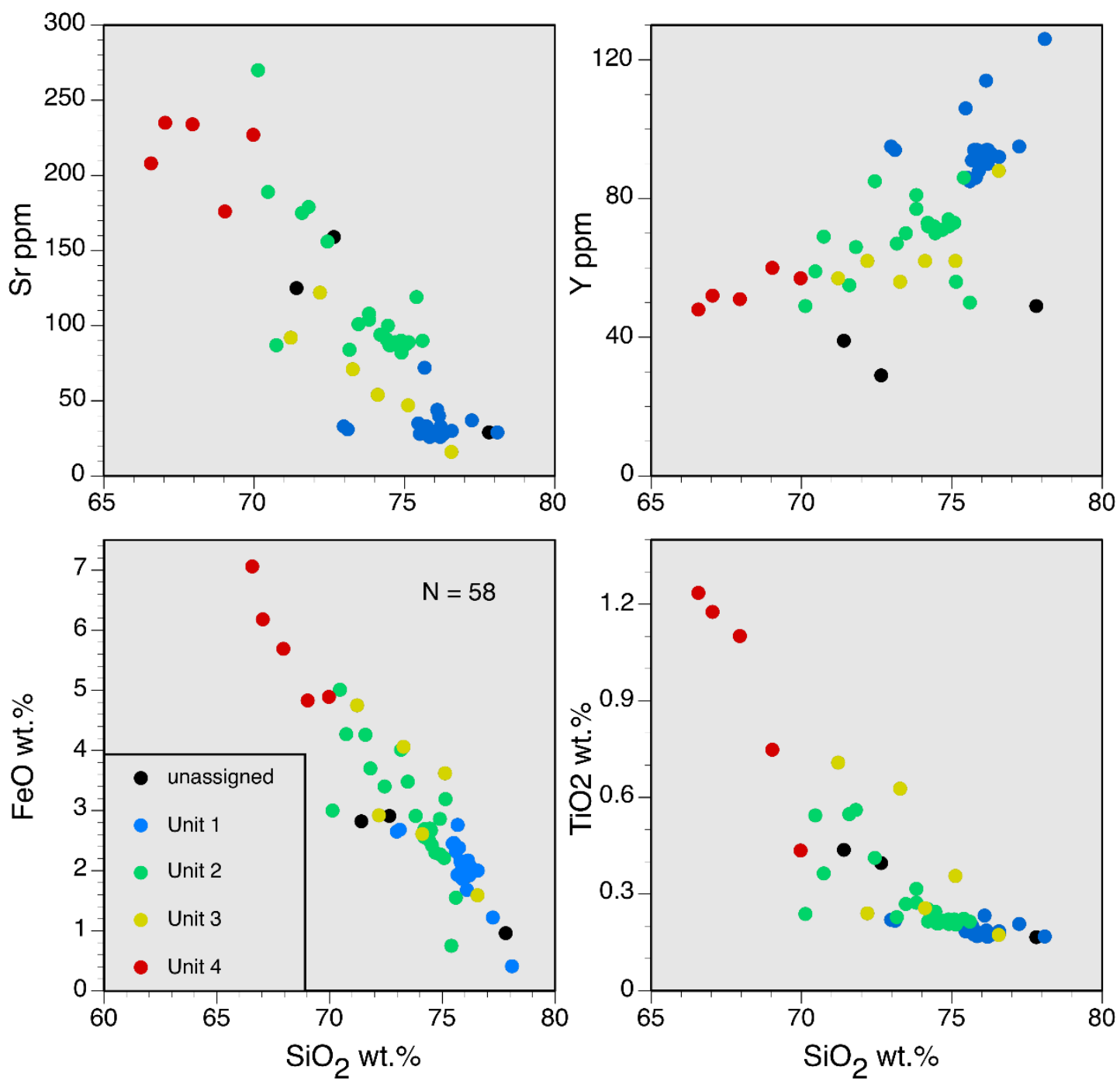

Figure 9. $\mathrm{Sr}, \mathrm{Y}, \mathrm{FeO}$ and $\mathrm{TiO}_{2}$ vs. $\mathrm{SiO}_{2}$ for all analyzed samples of Dinner Creek Tuff. Unit correlation based on feldspar composition, subtle chemical differences and available age dates. Unassigned samples are samples where feldspars have not been analyzed or other data are ambiguous (Streck, pers. comm., 2017). 


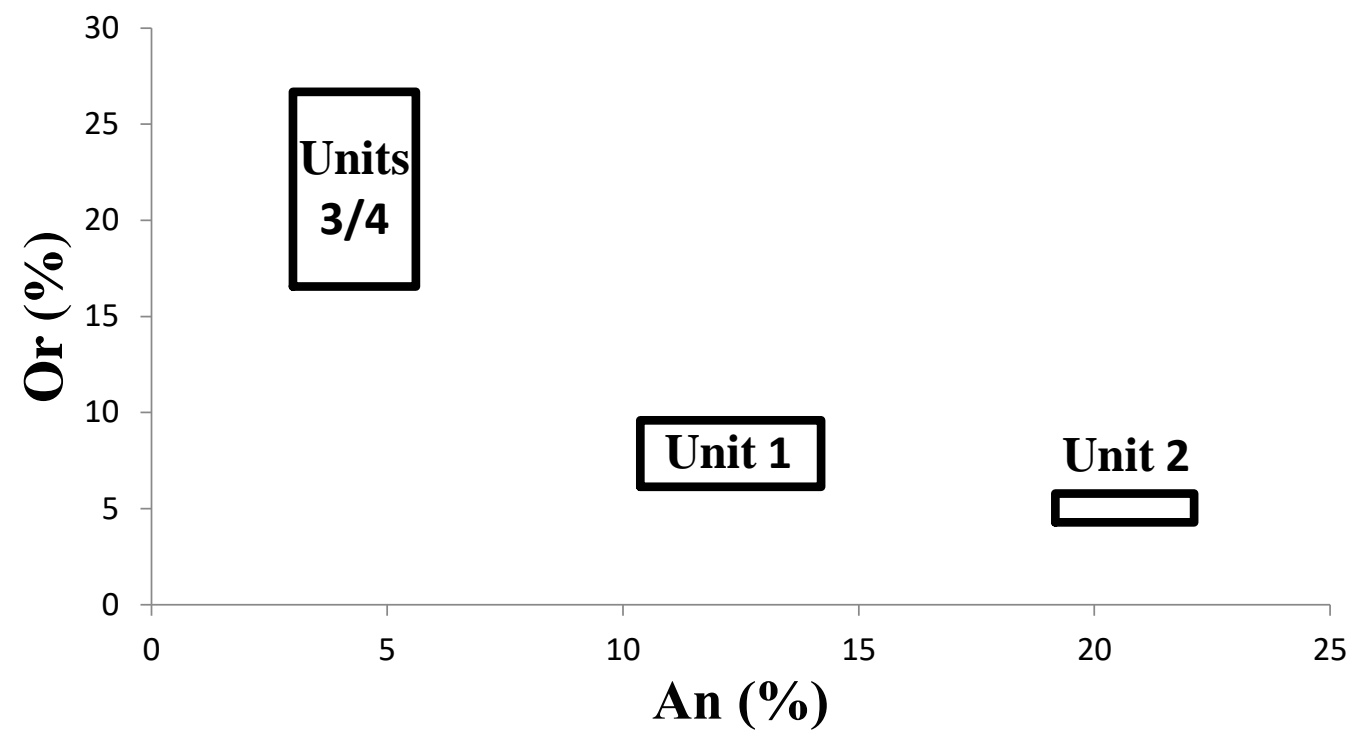

Figure 10. Anorthite vs. orthoclase diagram indicating unit correlating feldspar compositions from this study. 


\section{METHODS}

\section{Sample Selection}

Forty-six samples collected from outcrops across the extent area of the DIT were utilized for this study. Samples were collected mainly by Martin Streck with additional samples collected by Emily Hess, Aaron Steiner, Adam Large and one sample by Emily Cahoon (Figure 11).

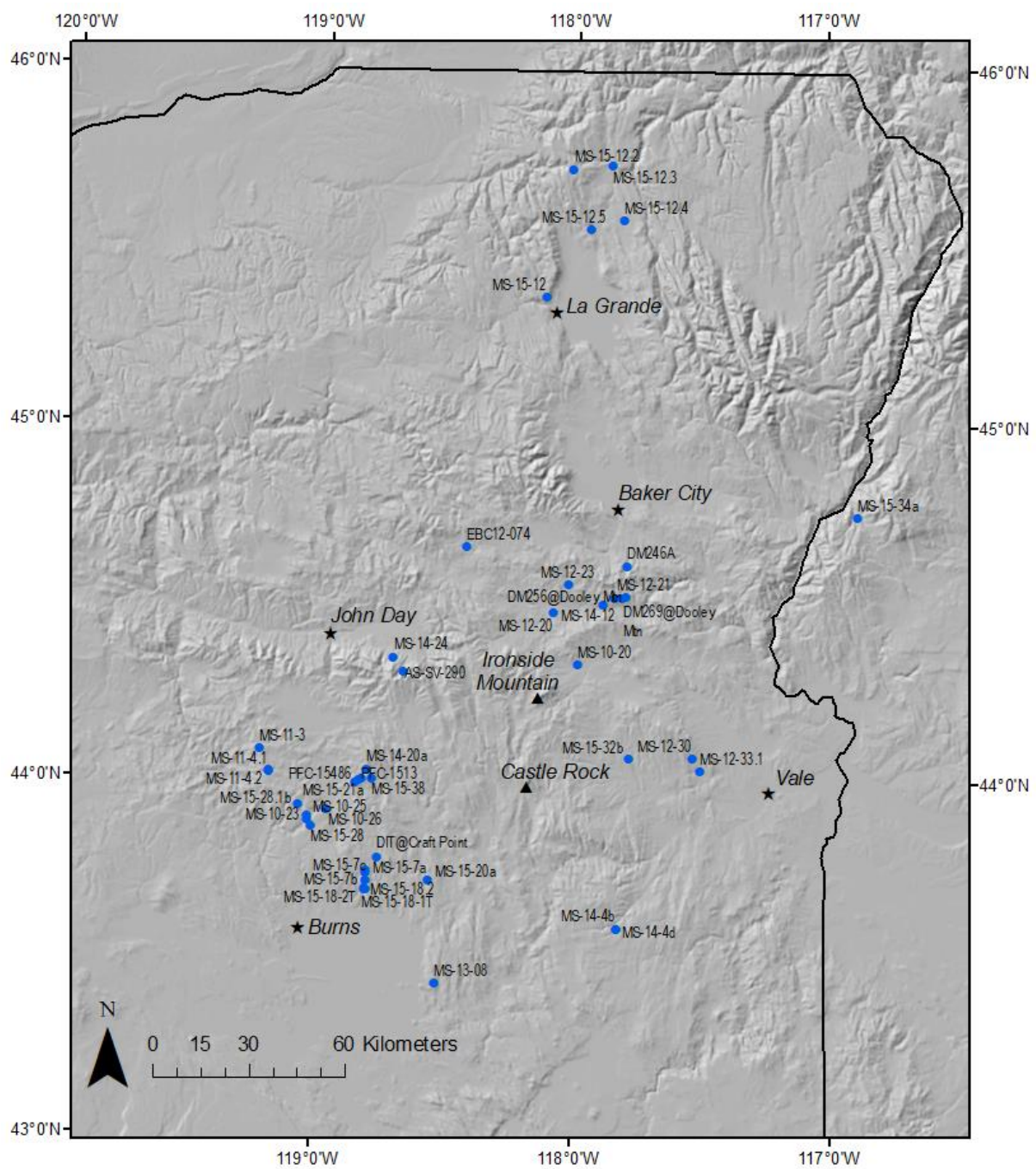

Figure 11. Locations and names of all samples associated with this study. Castle Rock and Ironside Mountain are the eruption sites for units 1 and 2, respectively. They also mark the approximate location of the DITEC. 


\section{Chemical Analysis}

\section{Sample Preparation}

Samples broken down with a rock pick before were further crushed in a mortar and pestle. Periodically during processing, the fine portions were sieved, and larger fragments returned to the mortar and pestle for further crushing to ensure minimal damage to the minerals. Processed samples were sequentially sieved into particle-size fractions of mesh sizes: >991 microns, 991-125 microns, $<125$ microns. Crushed material >991 microns underwent further crushing, until a majority to all the sample material passed through the 991-micron sieve. For the 991-125-micron size fractions, handpicking under the binocular microscope separated the feldspar crystals from the remaining sample. Selected feldspars crystals were then mounted in epoxy and the resulting plugs were progressively polished to 1 micron utilizing increasingly higher levels of grit prior to being covered with a 10-nm thick carbon coating.

\section{Electron microprobe analysis}

Chemical analyses of feldspar crystals were conducted using the CAMECA SX100 electron microprobe (EMP) at Oregon State University (OSU) controlled remotely from Portland State University. The following EMP parameters were used during analysis:

- Accelerating voltage of $15 \mathrm{KV}$

- $2 \mu \mathrm{m}$ beam 
Session calibrations utilized natural rhyolitic standards, mostly Smithsonian and USGS, both of which are long term standards used by the EMP lab at OSU. Each crystal was analyzed from 1 - 4 points per crystal, depending on time constraints. A maximum of 10 crystals per sample were analyzed during any one of the three EMP sessions. If less than 10 crystals were present, then the total number of crystals within that sample group were analyzed. Major elements analyzed included $\mathrm{Na}, \mathrm{Si}, \mathrm{Al}, \mathrm{Ca}$, $\mathrm{K}, \mathrm{Mg}$ and $\mathrm{Fe}$ in addition to the trace element $\mathrm{Ba}$. Due to instrumental malfunction during first session, Ba values determined during that session were not accurate and therefore discarded. Two of the plugs analyzed during that initial session were reanalyzed during later sessions due to low totals ( 72\% - 97\%).

\section{Unit Correlation}

The resulting feldspar data were used to calculate end member components compositions and generate anorthite vs. orthoclase diagrams to determine unit designation for units 1,2 and 3/4. Samples exhibiting single crystal anomalies where crystals plotted outside the unit designated by most of the sample crystals were excluded from unit designation but are discussed. Bulk rock geochemistry and lithology correlated samples plotted as units $3 / 4$ to their individual unit.

\section{Mapping}

ArcGIS was utilized for all maps. One third arc second digital elevation models (DEMs) from the USGS's National Map platform were downloaded and used to create the base for all maps in this study, due to the high resolution $(10 \mathrm{~m})$ and 
seamlessness derived from assorted source data that is processed to a single coordinate system and unit of vertical measure to represent the topographic bare-earth surface. Polygons for each unit were created and edited to enclose the locations of all samples correlated to a specific unit determined in this study and previous studies (Figure 12). The eruptive source or DITEC was included, but not centralized within each unit polygon. The polygon determining the total extent of the DIT traced the outer edges associated with individual unit polygons when all units were visible on one map. Individual unit and total extent areas were calculated within ArcGIS.

\section{Erupted tuff volumes and dense rock equivalent}

Total erupted tuff volumes and dense rock equivalents (DRE) were calculated based on the unit areas determined in this study. Associated formulas, thicknesses and densities utilized in the calculations are discussed further in the corresponding discussion section. 


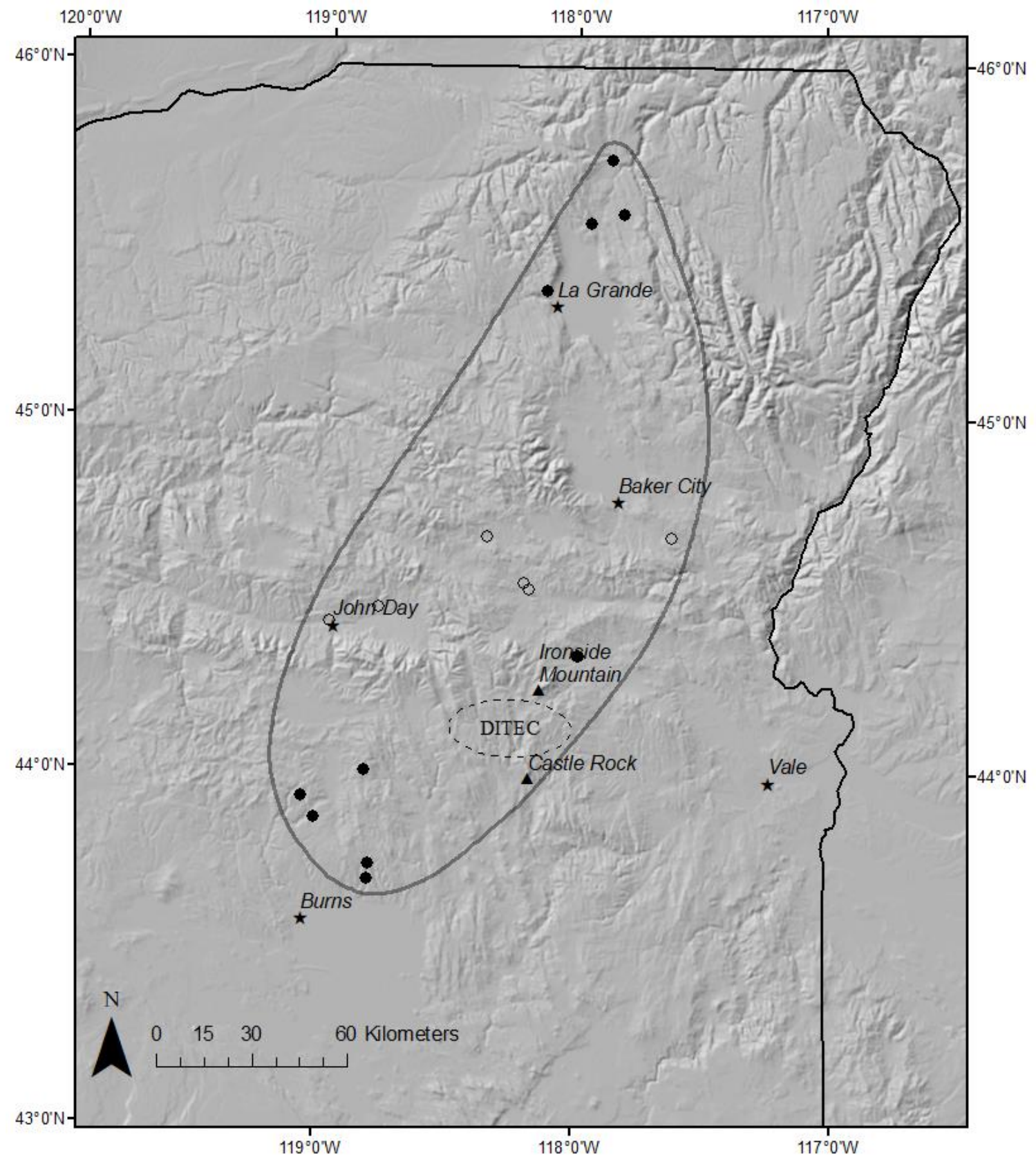

Figure 12. Example of how each polygon was created to enclose the sample locations while including Castle Rock (unit 1 source), Ironside Mountain (unit 2 source) or the DITEC from Figure 2, but not centralizing the eruptive sources within the individual polygons. Solid dots indicate data from this study and hollow dots are data from Streck et al. (2015). 


\section{RESULTS}

Results from this study on the DIT are organized into four different sections: 1) unit designation utilizing EMP feldspar data from this study combined with bulk tuff data from previous DIT studies, 2) unit designated feldspar geochemistry, 3) extent maps created from GPS coordinates from this study combined with previous DIT studies and 4) non-unit designating individual feldspar crystal classification.

\section{Unit Designation}

Of the 47 samples selected for this study, 37 samples yielded geochemical data sufficient for analysis (Figure 13). The remaining 10 samples either did not yield feldspar for analysis or analysis indicated non-unit designating feldspars.

\section{Feldspar Crystal EMP Analysis}

All samples containing unit designating feldspars were categorized by feldspar end member concentrations of anorthite vs. orthoclase using following ranges for units 1 to 4: unit $1\left(\mathrm{An}_{10-15}, \mathrm{Or}_{6-10}\right)$, unit $2\left(\mathrm{An}_{19-23}, \mathrm{Or}_{4-6}\right)$ and units $3 / 4\left(\mathrm{An}_{3-6}, \mathrm{Or}_{16-27}\right)$ which are shown in Figures 14a and 14b. Samples were spilt between the two diagrams for ease of reading. While the anorthite and orthoclase concentrations in units 3 and 4 are clearly distinct from units 1 and 2, they are indistinguishable from each other based on anorthite vs. orthoclase alone. Appendices A-D contain end member concentrations and standard deviations for the individual samples as well as individual crystals by unit. Table 1 contains end member concentration statistics by unit. 


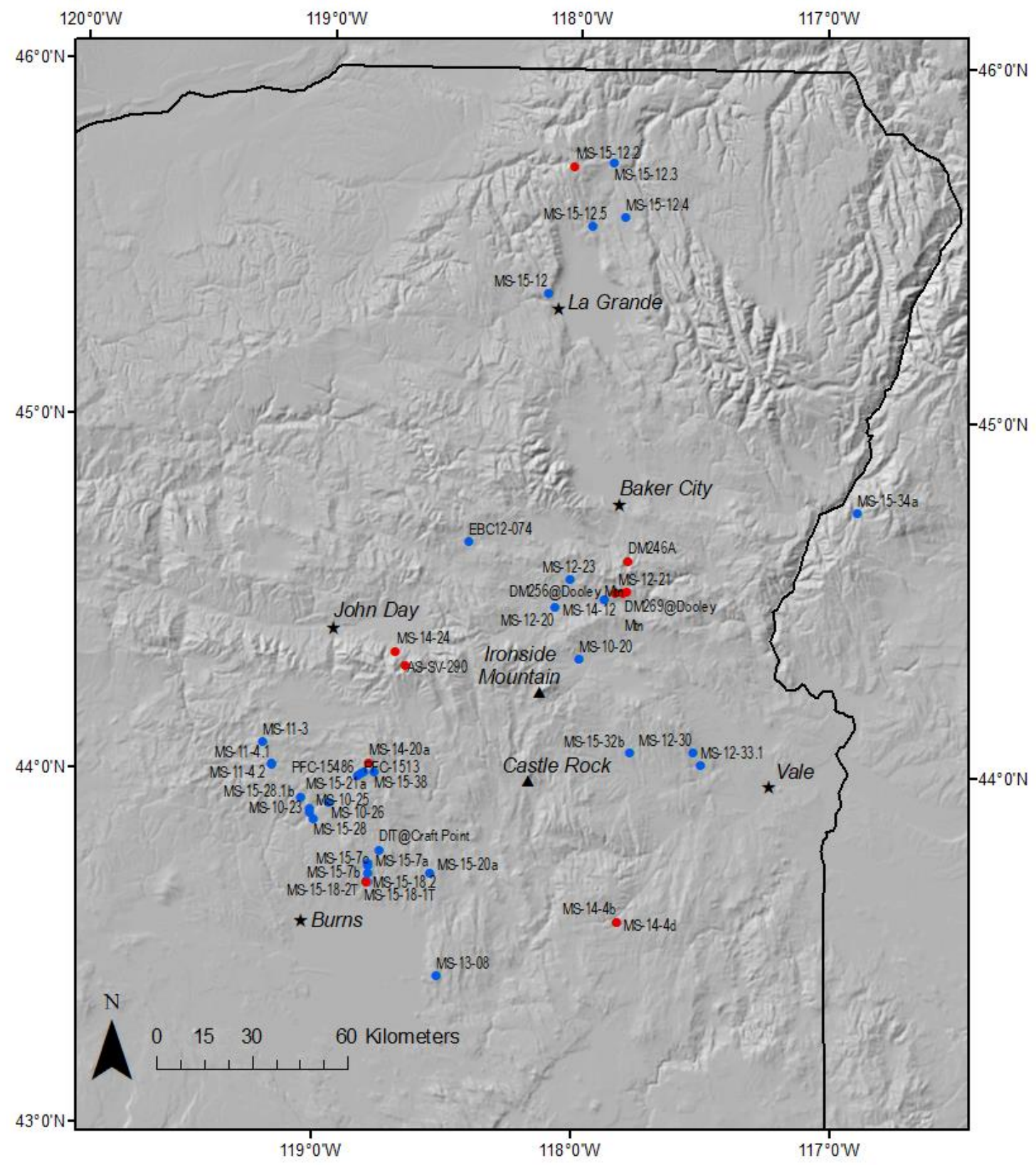

Figure 13. The map shows the locations of the samples utilized in this study. Blue dots refer to samples that yielded sufficient mineral chemical data for unit correlation. Red dots refer to samples that either lacked feldspar for chemical analysis or analysis indicated non-unit designating feldspars. Castle Rock and Ironside Mountain are the eruption sites for units 1 and 2, respectively. They also mark the north/south boundaries of the DITEC. 

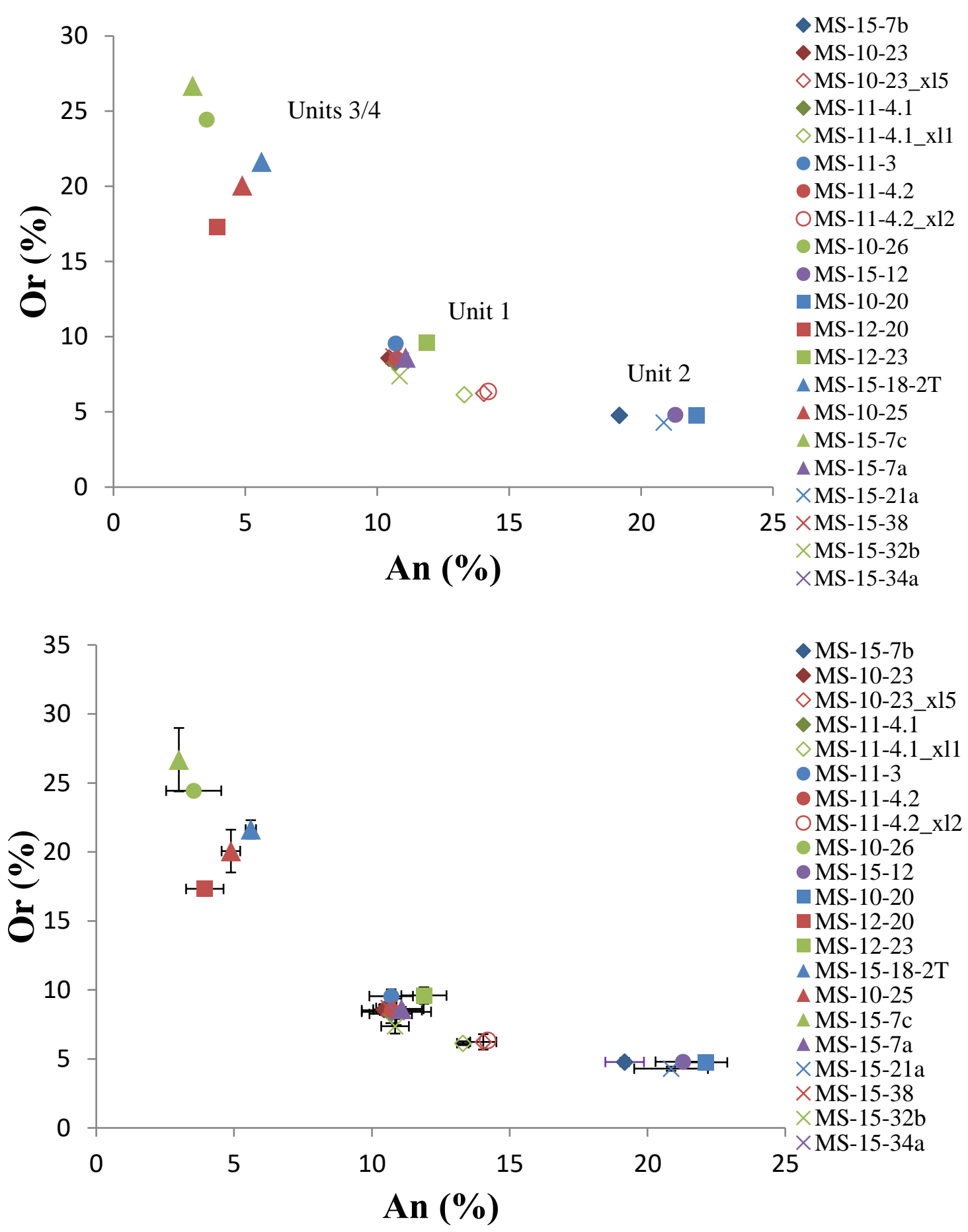

Figure 14a. Upper-anorthite vs. orthoclase diagrams correlating samples in this study to the cooling units. Lower - same diagram, but with error bars representing the individual sample standard deviations from Appendices A-D. 

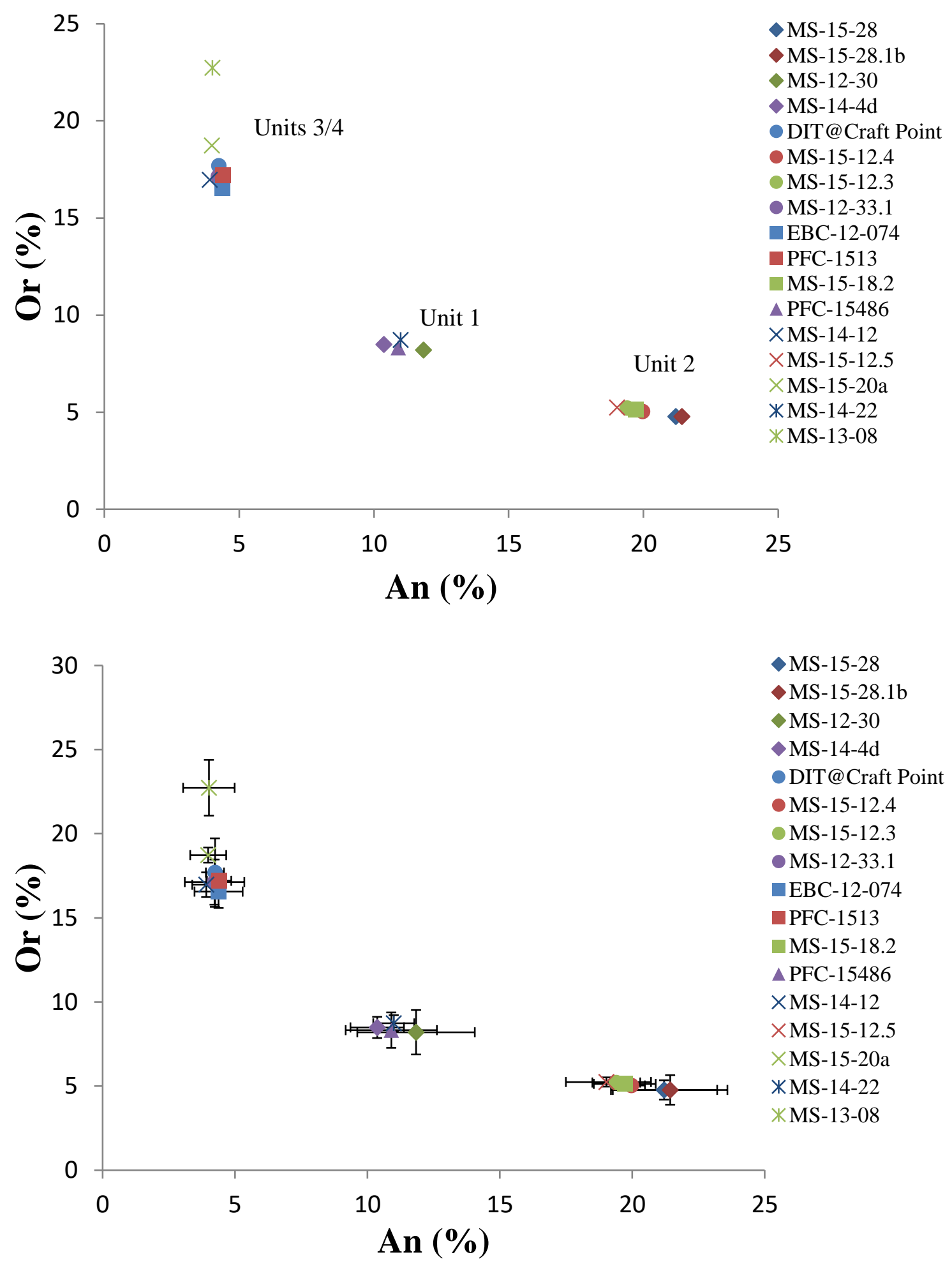

Figure 14b. Upper-anorthite vs. orthoclase diagrams correlating samples in this study to the cooling units. Lower-same diagram, but with error bars representing the individual sample standard deviations from Appendices A-D. 
Table 1. Average feldspar end member component composition statistics by unit.

\begin{tabular}{|c|c|c|c|c|c|c|c|}
\hline Unit & An & $\mathbf{A b}$ & Or & Unit & An & $\mathbf{A b}$ & Or \\
\hline 1 & & & & 3 & & & \\
\hline Average & 11.5 & 80.4 & 8.1 & Average & 4.1 & 77.5 & 18.3 \\
\hline Minimum & 10.4 & 78.5 & 6.1 & Minimum & 3.9 & 73.3 & 16.6 \\
\hline Maximum & 14.2 & 81.8 & 9.6 & Maximum & 4.4 & 79.1 & 22.7 \\
\hline $\begin{array}{l}\text { Std. Dev. } \\
2\end{array}$ & 1.3 & 0.8 & 1.1 & $\begin{array}{l}\text { Std. Dev. } \\
4\end{array}$ & 0.2 & 2.8 & 2.9 \\
\hline Average & 20.9 & 74.3 & 4.8 & Average & 4.2 & 75.3 & 20.5 \\
\hline Minimum & 19.2 & 72.8 & 4.0 & Minimum & 3.0 & 70.3 & 17.2 \\
\hline Maximum & 22.7 & 76.1 & 5.8 & Maximum & 5.6 & 78.8 & 26.7 \\
\hline Std. Dev. & 1.2 & 1.0 & 0.5 & Std. Dev. & 0.8 & 3.3 & 3.5 \\
\hline
\end{tabular}

The 37 samples designations are as follows: unit $1-13$ samples, unit $2-11$ samples, units 3/4-13 samples. MS-10-23, MS-11-4.1 and MS-14-4.2 exhibited single crystal anomalies where a single crystal from each sample contained higher anorthite $(\sim 13.2 \%-\sim 14.5 \%$ vs. $\sim 9 \%-\sim 13 \%)$ and lower orthoclase concentrations $(\sim 6.0 \%-\sim 6.4 \%$ vs. $\sim 7 \%-\sim 10 \%)$ than the sample average.

\section{Unit 3 and 4 designations}

\section{Lithology and Bulk Tuff Data}

Lithology and bulk tuff composition can be used to distinguish unit 3 from unit

4. In appearance, the gray color of unit 3 varies with welding but samples with a similar degree of welding to unit 4 samples, tend to be lighter than unit 4 samples (Table 2). Darker gray unit 3 samples display a higher degree of welding and appear glassy (Table 2). Unit 4 is the only unit to contain vesicular globules of tholeiitic andesite glass (Figure 8). Silica content determined by the bulk rock analyses from Streck et al. (2015) differentiates the rhyolitic $\left(>70 \% \mathrm{SiO}_{2}\right)$ unit 3 from the dacitic 
$\left(<70 \% \mathrm{SiO}_{2}\right)$ unit 4 for five of the samples in this study (Table 2). $\mathrm{Nb}$ and $\mathrm{Sr}$ concentrations also differentiate the units (Figure 15). Appendix F contains all bulk rock data available for unit designated samples in this study as well as data for previously correlated samples.

Table 2. Unit $3 / 4$ designations

\begin{tabular}{|c|c|c|c|c|c|}
\hline Sample & $\begin{array}{c}\mathrm{SiO}_{2} \\
\text { (wt. \%) }\end{array}$ & Description & Sample & $\begin{array}{c}\mathrm{SiO}_{2} \\
\text { (wt. \%) }\end{array}$ & Description \\
\hline Unit 3 & & & Unit 4 & & \\
\hline MS-10-26 & 76.6 & gray & MS-12-20 & 67.0 & dark gray \\
\hline EBC12-074 & 75.0 & gray, glassy & MS-15-20a & 69.0 & dark gray \\
\hline MS-12-33.1 & 75.1 & light gray & MS-15-7c & N/A & dark gray \\
\hline MS-14-22 & N/A & light gray & DIT@Craft Point & N/A & dark gray \\
\hline MS-13-08 & N/A & light gray & MS-15-18-2T & N/A & dark gray \\
\hline PFC-1513 & N/A & gray, glassy & & & \\
\hline MS-10-25 & N/A & gray & & & \\
\hline
\end{tabular}

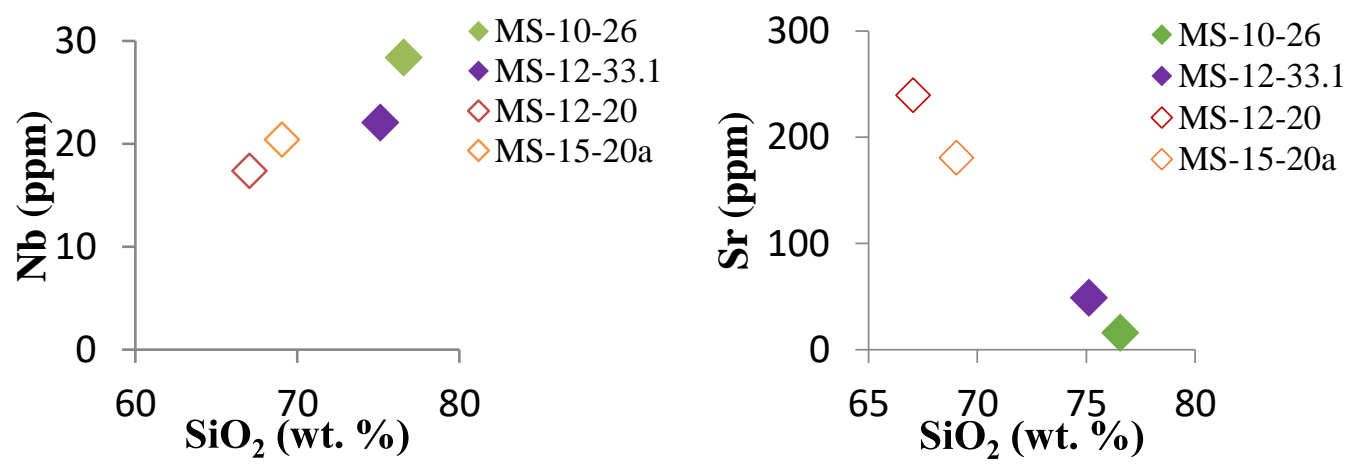

Figure 15. $\mathrm{Nb}$ and $\mathrm{Sr}$ vs. $\mathrm{SiO}_{2}$ diagrams from the bulk rock data also differentiate unit 3 from unit 4 . Solid shapes correlate to unit 3 and hollow shapes to unit 4.

\section{Unit designating feldspar major element geochemistry}

\section{Unit 1}

Table 3 contains the average weight percent concentrations of the major elements for unit 1 designated feldspar crystals. Appendix A contains individual 
crystal chemical data. With a few exceptions, the feldspars analyzed displayed little chemical variation among major elements. $\mathrm{SiO}_{2}$ concentrations in MS-11-4.2 and PFC-15486 were the only samples to exceed one standard deviation. Iron concentrations were highest in sample PFC15486, $\sim 0.33 \%$ and the highest concentration among all the units. Magnesium is generally below detection limit in all units. MS-11-4.1_xl1 and MS-10-23_xl5 contained the lowest barium concentrations, $\sim 0.26 \%$ and $\sim 0.28 \%$ respectively. Compared to other units, unit 1 contains the highest $\mathrm{NaO}(\sim 9.61 \%)$ and $\mathrm{FeO} *(\sim 0.33 \%)$ concentrations.

Table 3. Unit 1 average EMP major element feldspar chemical composition by sample.

\begin{tabular}{|c|c|c|c|c|c|c|c|}
\hline Sample & $\begin{array}{c}\text { Number } \\
\text { of } \\
\text { Crystals }\end{array}$ & $\begin{array}{c}\mathrm{SiO}_{2} \\
(\text { wt. \%) }\end{array}$ & $\begin{array}{l}\mathrm{SiO}_{2} \\
\text { Std. }\end{array}$ & $\begin{array}{c}\mathrm{Al}_{2} \mathbf{O}_{3} \\
(w t . \%)\end{array}$ & $\begin{array}{c}\mathrm{Al}_{2} \mathbf{O}_{3} \\
\text { Stdd }\end{array}$ & $\begin{array}{c}\text { FeO* } \\
(\text { wt. \%) }\end{array}$ & $\begin{array}{c}\text { FeO* } \\
\text { Std. }\end{array}$ \\
\hline MS-10-23 & 6 & 64.13 & 0.3 & 22.13 & 0.20 & 0.28 & 0.04 \\
\hline MS-10-23_x15 & 1 & 63.46 & 0.2 & 22.73 & 0.13 & 0.25 & 0.07 \\
\hline MS-11-4.1 ${ }^{1}$ & 5 & 63.92 & 0.4 & 22.32 & 0.27 & 0.25 & 0.04 \\
\hline MS-11-4.1_x11 ${ }^{1,2}$ & 1 & 63.33 & 0.2 & 22.54 & 0.02 & 0.29 & 0.07 \\
\hline MS-11-3 & 4 & 63.65 & 0.8 & 21.37 & 0.40 & 0.27 & 0.04 \\
\hline MS-11-4.2 & 4 & 65.44 & 1.1 & 21.22 & 0.26 & 0.25 & 0.03 \\
\hline MS-11-4.2_x12 & 1 & 64.70 & N/A & 21.92 & N/A & 0.24 & N/A \\
\hline MS-12-23 & 7 & 67.46 & 0.6 & 21.50 & 0.24 & 0.25 & 0.03 \\
\hline MS-15-7a & 7 & 66.78 & 0.6 & 21.47 & 0.12 & 0.25 & 0.02 \\
\hline MS-15-38 & 6 & 66.43 & 0.4 & 20.79 & 0.22 & 0.26 & 0.02 \\
\hline MS-15-32b & 7 & 66.35 & 0.4 & 20.98 & 0.44 & 0.22 & 0.05 \\
\hline MS-15-34a & 6 & 65.21 & 0.8 & 21.05 & 0.43 & 0.25 & 0.04 \\
\hline MS-12-30 & 9 & 65.76 & 0.8 & 21.66 & 0.44 & 0.24 & 0.03 \\
\hline MS-14-4d & 10 & 65.56 & 0.7 & 21.56 & 0.27 & 0.24 & 0.04 \\
\hline PFC-15486 & 10 & 65.35 & 1.2 & 21.24 & 0.49 & 0.33 & 0.29 \\
\hline MS-14-22 & 1 & 65.54 & 0.2 & 21.24 & 0.17 & 0.25 & 0.02 \\
\hline
\end{tabular}


Table 3 continued.

\begin{tabular}{lccccc}
\hline \hline Sample & $\begin{array}{c}\text { Number of } \\
\text { Crystals }\end{array}$ & $\begin{array}{c}\mathbf{C a O} \\
(\mathbf{w t} \text { \% })\end{array}$ & $\begin{array}{c}\mathbf{C a O} \\
\text { Std. }\end{array}$ & $\begin{array}{c}\mathbf{N a}_{2} \mathbf{O} \\
(\mathbf{w t} \%)\end{array}$ & $\begin{array}{c}\mathbf{N a}_{2} \mathbf{O} \\
\text { Std. }\end{array}$ \\
\hline MS-10-23 ${ }^{1}$ & 6 & 2.17 & 0.09 & 9.35 & 0.11 \\
MS-10-23_x15 $^{1,2}$ & 1 & 2.94 & 0.11 & 9.25 & 0.04 \\
MS-11-4.1 $^{1}$ & 5 & 2.23 & 0.17 & 9.39 & 0.13 \\
MS-11-4.1_x11 & 1 & 2.86 & 0.02 & 9.61 & 0.27 \\
MS-11-3 & 4 & 2.17 & 0.14 & 8.95 & 0.20 \\
MS-11-4.2 & 4 & 2.13 & 0.20 & 8.92 & 0.18 \\
MS-11-4.2_x12 & 1 & 2.88 & N/A & 8.93 & N/A \\
MS-12-23 & 7 & 2.14 & 0.18 & 8.95 & 0.11 \\
MS-15-7a & 7 & 2.16 & 0.12 & 8.68 & 0.29 \\
MS-15-38 & 6 & 2.08 & 0.12 & 8.78 & 0.18 \\
MS-15-32b & 7 & 2.13 & 0.12 & 8.92 & 0.18 \\
MS-15-34a & 6 & 2.13 & 0.25 & 8.77 & 0.12 \\
MS-12-30 & 9 & 2.33 & 0.45 & 8.73 & 0.17 \\
MS-14-4d & 10 & 2.23 & 0.19 & 8.81 & 0.15 \\
PFC-15486 & 10 & 2.32 & 0.34 & 8.69 & 0.40 \\
MS-14-22 & 1 & 2.21 & 0.15 & 8.94 & 0.11 \\
\hline
\end{tabular}


Table 3 continued.

\begin{tabular}{|c|c|c|c|c|c|}
\hline Sample & $\begin{array}{c}\text { Number } \\
\text { of } \\
\text { Crystals }\end{array}$ & $\begin{array}{c}\mathrm{K}_{2} \mathrm{O} \\
\text { (wt. \%) }\end{array}$ & $\begin{array}{l}\mathrm{K}_{2} \mathrm{O} \\
\text { Std. }\end{array}$ & $\begin{array}{c}\text { BaO } \\
\text { (wt. \%) }\end{array}$ & $\begin{array}{l}\text { BaO } \\
\text { Std. }\end{array}$ \\
\hline MS-10-23 ${ }^{1}$ & 6 & 1.50 & 0.06 & 0.43 & 0.03 \\
\hline MS-10-23_x15 $5^{1,2}$ & 1 & 1.09 & 0.09 & 0.28 & 0.03 \\
\hline MS-11-4.1 $1^{1}$ & 5 & 1.45 & 0.11 & 0.43 & 0.04 \\
\hline MS-11-4.1_x11 ${ }^{1,2}$ & 1 & 1.11 & 0.00 & 0.26 & 0.02 \\
\hline MS-11-3 & 4 & 1.39 & 0.09 & N/A & N/A \\
\hline MS-11-4.2 & 4 & 1.42 & 0.10 & N/A & N/A \\
\hline MS-11-4.2_x12 & 1 & 1.08 & N/A & N/A & N/A \\
\hline MS-12-23 & 7 & 1.45 & 0.09 & 0.48 & 0.04 \\
\hline MS-15-7a & 7 & 1.41 & 0.03 & N/A & N/A \\
\hline MS-15-38 & 6 & 1.43 & 0.05 & N/A & N/A \\
\hline MS-15-32b & 7 & 1.39 & 0.07 & N/A & N/A \\
\hline MS-15-34a & 6 & 1.38 & 0.15 & N/A & N/A \\
\hline MS-12-30 & 9 & 1.35 & 0.21 & 0.42 & 0.08 \\
\hline MS-14-4d & 10 & 1.39 & 0.10 & 0.44 & 0.03 \\
\hline PFC-15486 & 10 & 1.35 & 0.16 & 0.43 & 0.07 \\
\hline MS-14-22 & 1 & 1.47 & 0.09 & 0.47 & 0.03 \\
\hline
\end{tabular}

\section{Unit 2}

Table 4 contains the average weight percent concentrations of the major elements for unit 2 designated feldspar crystals. Appendix B contains individual crystal chemical data. Silica content was slightly lower than unit 1 with weight percent $\mathrm{SiO}_{2}$ ranging from $\sim 61.21 \%$ to $\sim 64.38 \%$. The feldspars analyzed displayed little chemical variation among the remainder of the major elements with only $\mathrm{SiO}_{2}$ for sample MS-15-28.1b exceeding one standard deviation. Unlike unit 1, no individual sample showed significant contrast in major element concentrations compared to the 
other samples. Sample MS-15-7b contained the lowest $\mathrm{SiO}_{2}(\sim 61.21 \%), \mathrm{Al}_{2} \mathrm{O}_{3}$ $(\sim 21.0 \%), \mathrm{FeO}^{*}(\sim 0.22 \%), \mathrm{Na}_{2} \mathrm{O}(\sim 7.86)$ and $\mathrm{BaO}(\sim 0.20 \%)$ concentrations. No individual sample contained the highest elemental concentrations across the major element concentrations. Compared to other units, unit 2 contains the highest $\mathrm{Al}_{2} \mathrm{O}_{3}$ $(\sim 23.64 \%)$ and $\mathrm{CaO}(\sim 4.41 \%)$ concentrations and the lowest $\mathrm{SiO}_{2}(\sim 61.21 \%), \mathrm{K}_{2} \mathrm{O}$ $(\sim 0.75 \%)$ and $\mathrm{BaO}(\sim 0.20 \%)$ concentrations.

Table 4. Unit 2 average EMP major element feldspar chemical composition by sample.

\begin{tabular}{lccccccc}
\hline \hline Sample & $\begin{array}{c}\text { Number } \\
\text { of } \\
\text { Crystals }\end{array}$ & $\begin{array}{c}\mathrm{SiO}_{2} \\
(\mathbf{w t .} \%)\end{array}$ & $\begin{array}{c}\mathrm{SiO}_{2} \\
\text { Std. }\end{array}$ & $\begin{array}{c}\mathrm{Al}_{2} \mathbf{O}_{3} \\
(\mathbf{w t .} \%)\end{array}$ & $\begin{array}{c}\mathrm{Al}_{2} \mathbf{O}_{3} \\
\text { Std }\end{array}$ & $\begin{array}{c}\mathbf{F e O}^{*} \\
(\mathbf{w t .} \%)\end{array}$ & $\begin{array}{c}\mathbf{F e O}^{*} \\
\text { Std. }\end{array}$ \\
\hline MS-15-7b & 7 & 61.21 & 0.21 & 24.23 & 0.11 & 0.25 & 0.04 \\
MS-15-12 & 1 & 62.27 & 1.20 & 22.48 & 0.25 & 0.23 & 0.03 \\
MS-10-20 & 2 & 63.92 & 0.84 & 23.64 & 0.32 & 0.23 & 0.04 \\
MS-15-21a & 7 & 63.41 & 0.56 & 22.77 & 0.14 & 0.24 & 0.04 \\
MS-15-28 & 10 & 64.33 & 0.69 & 23.22 & 0.45 & 0.24 & 0.04 \\
MS-15-28.1b & 1 & 63.36 & 1.06 & 23.06 & 0.55 & 0.31 & 0.02 \\
MS-15-12.4 & 3 & 64.38 & 0.45 & 23.00 & 0.09 & 0.23 & 0.04 \\
MS-12-12.3 & 5 & 64.05 & 0.47 & 22.76 & 0.22 & 0.23 & 0.03 \\
MS-15-18.2 & 6 & 62.33 & 0.91 & 22.62 & 0.29 & 0.23 & 0.03 \\
MS-15-12.5 & 2 & 63.96 & 0.58 & 22.75 & 0.32 & 0.23 & 0.02 \\
\hline
\end{tabular}


Table 4 continued.

\begin{tabular}{lccccc}
\hline \hline Sample & $\begin{array}{c}\text { Number } \\
\text { of } \\
\text { Crystals }\end{array}$ & $\begin{array}{c}\mathbf{C a O} \\
(\text { wt. \%) }\end{array}$ & $\begin{array}{c}\mathbf{C a O} \\
\text { Std. }\end{array}$ & $\begin{array}{c}\mathbf{N a}_{2} O \\
(\text { wt. \%) }\end{array}$ & $\begin{array}{c}\mathbf{N a}_{2} O \\
\text { Std. }\end{array}$ \\
\hline MS-15-7b & 7 & 4.13 & 0.14 & 7.86 & 0.15 \\
MS-15-12 & 1 & 3.88 & 0.18 & 8.13 & 0.24 \\
MS-10-20 & 2 & 4.41 & 0.21 & 8.09 & 0.01 \\
MS-15-21a & 7 & 4.10 & 0.25 & 8.16 & 0.18 \\
MS-15-28 & 10 & 4.20 & 0.38 & 8.15 & 0.24 \\
MS_15_28.1b & 1 & 4.28 & 0.61 & 8.06 & 0.18 \\
MS-15-12.4 & 3 & 4.03 & 0.08 & 8.39 & 0.16 \\
MS-12-12.3 & 5 & 3.88 & 0.18 & 8.36 & 0.15 \\
MS-15-18.2 & 6 & 3.87 & 0.20 & 8.19 & 0.20 \\
MS-15-12.5 & 2 & 3.84 & 0.32 & 8.54 & 0.27 \\
\hline
\end{tabular}

Table 4 continued.

\begin{tabular}{lccccc}
\hline \hline Sample & $\begin{array}{c}\text { Number } \\
\text { of } \\
\text { Crystals }\end{array}$ & $\begin{array}{c}\mathbf{K}_{2} \mathbf{O} \\
(\mathbf{w t} . \%)\end{array}$ & $\begin{array}{c}\mathbf{K}_{2} \mathbf{O} \\
\text { Std. }\end{array}$ & $\begin{array}{c}\text { BaO } \\
(\mathbf{w t .} \%)\end{array}$ & $\begin{array}{c}\text { BaO } \\
\text { Std. }\end{array}$ \\
\hline MS-15-7b & 7 & 0.75 & 0.05 & 0.20 & 0.02 \\
MS-15-12 & 1 & 0.84 & 0.04 & N/A & N/A \\
MS-10-20 & 2 & 0.79 & 0.01 & 0.21 & 0.03 \\
MS-15-21a & 7 & 0.81 & 0.03 & N/A & N/A \\
MS-15-28 & 10 & 0.79 & 0.10 & 0.23 & 0.03 \\
MS_15_28.1b & 1 & 0.97 & 0.11 & 0.25 & 0.02 \\
MS-15-12.4 & 3 & 0.85 & 0.02 & 0.24 & 0.02 \\
MS-12-12.3 & 5 & 0.87 & 0.04 & 0.25 & 0.04 \\
MS-15-18.2 & 6 & 0.84 & 0.05 & 0.23 & 0.04 \\
MS-15-12.5 & 2 & 0.89 & 0.04 & 0.27 & 0.04 \\
\hline 1. Normalized sample \\
2. Data averaged from two individual samples.
\end{tabular}

\section{Unit 3}

Table 5 contains the average weight percent concentrations of the major elements for unit 3 designated feldspar crystals. Appendix $\mathrm{C}$ contains individual 
crystal chemical data. Weight percent $\mathrm{SiO}_{2}$ ranges from $\sim 62.11 \%$ to $\sim 68.28 \%$, the largest variation among the major chemical elements. With a few exceptions, the feldspars analyzed displayed little chemical variation among major elements. MS-1308 contained the lowest $\mathrm{BaO}$ concentration at $\sim 0.56 \%$ and highest $\mathrm{K}_{2} \mathrm{O}$ concentration at $\sim 3.8 \%$. No individual sample contained the highest or lowest elemental concentrations across the major element concentrations as well as across the units.

Table 5. Unit 3 average EMP major element feldspar chemical composition by sample.

\begin{tabular}{lccccccc}
\hline \hline Sample & $\begin{array}{c}\text { Number } \\
\text { of } \\
\text { Crystals }\end{array}$ & $\begin{array}{c}\mathrm{SiO}_{2} \\
(\mathbf{w t .} \%)\end{array}$ & $\begin{array}{c}\mathrm{SiO}_{2} \\
\text { Std. }\end{array}$ & $\begin{array}{c}\mathrm{Al}_{2} \mathbf{O}_{3} \\
(\text { wt. \%) }\end{array}$ & $\begin{array}{c}\mathrm{Al}_{2} \mathbf{O}_{3} \\
\text { Std }\end{array}$ & $\begin{array}{c}\mathbf{F e O}^{*} \\
\text { (wt. \%) }\end{array}$ & $\begin{array}{c}\mathbf{F e O}^{*} \\
\text { Std. }\end{array}$ \\
\hline MS-12-33.1* & 11 & 67.98 & 0.84 & 20.31 & 0.31 & 0.24 & 0.03 \\
EBC-12-074 & 10 & 62.12 & 0.51 & 19.83 & 0.25 & 0.22 & 0.03 \\
MS-14-12 & 10 & 67.18 & 0.64 & 20.54 & 0.23 & 0.21 & 0.03 \\
MS-13-08 & 10 & 66.61 & 0.56 & 20.01 & 0.22 & 0.28 & 0.04 \\
MS-10-26 & 1 & 65.88 & N/A & 20.07 & N/A & 0.24 & N/A \\
MS-10-25 & 7 & 68.23 & 0.64 & 20.36 & 0.19 & 0.24 & 0.03 \\
PFC-1513 & 10 & 63.58 & 0.81 & 20.13 & 0.24 & 0.23 & 0.05 \\
\hline
\end{tabular}

Table 5 continued.

\begin{tabular}{lccccc}
\hline \hline Sample & $\begin{array}{c}\text { Number } \\
\text { of } \\
\text { Crystals }\end{array}$ & $\begin{array}{c}\mathrm{CaO} \\
(\mathbf{w t .} \%)\end{array}$ & $\begin{array}{c}\mathrm{CaO} \\
\text { Std. }\end{array}$ & $\begin{array}{c}\mathbf{N a}_{2} \mathbf{O} \\
(\mathbf{w t} . \%)\end{array}$ & $\begin{array}{c}\mathrm{Na}_{2} \mathbf{O} \\
\text { Std. }\end{array}$ \\
\hline MS-12-33.1* & 11 & 0.82 & 0.22 & 8.60 & 0.15 \\
EBC-12-074 & 10 & 0.84 & 0.17 & 8.39 & 0.16 \\
MS-14-12 & 10 & 0.79 & 0.11 & 8.91 & 0.15 \\
MS-13-08 & 10 & 0.80 & 0.19 & 8.13 & 0.16 \\
MS-10-26 & 1 & 0.69 & N/A & 7.78 & N/A \\
MS-10-25 & 7 & 0.84 & 0.09 & 8.17 & 0.63 \\
PFC-1513 & 10 & 0.85 & 0.09 & 8.39 & 0.11 \\
\hline
\end{tabular}


Table 5 continued.

\begin{tabular}{lccccc}
\hline \hline Sample & $\begin{array}{c}\text { Number } \\
\text { of } \\
\text { Crystals }\end{array}$ & $\begin{array}{c}\mathbf{K}_{2} \mathbf{O} \\
(\mathbf{w t .} \%)\end{array}$ & $\begin{array}{c}\mathbf{K}_{2} \mathbf{O} \\
\text { Std. }\end{array}$ & $\begin{array}{c}\mathbf{B a O} \\
(\mathbf{w t} . \%)\end{array}$ & $\begin{array}{c}\text { BaO } \\
\text { Std. }\end{array}$ \\
\hline MS-12-33.1* & 11 & 2.84 & 0.23 & 0.95 & 0.16 \\
EBC-12-074 & 10 & 2.66 & 0.16 & 0.91 & 0.12 \\
MS-14-12 & 10 & 2.89 & 0.11 & 0.98 & 0.07 \\
MS-13-08 & 10 & 3.81 & 0.30 & 0.56 & 0.09 \\
MS-10-26 & 1 & 3.99 & N/A & N/A & N/A \\
MS-10-25 & 7 & 2.89 & 0.08 & N/A & N/A \\
PFC-1513 & 10 & 2.78 & 0.09 & 0.93 & 0.07 \\
\hline *Data averaged from two individual samples. \\
\hline \multicolumn{7}{l}{}
\end{tabular}

\section{Unit 4}

Table 6 contains the average weight percent concentrations of the major elements for unit 4 designated feldspar crystals. Appendix D contains individual crystal chemical data. Weight percent $\mathrm{SiO}_{2}$ ranges from $\sim 66.79 \%$ to $\sim 68.53 \%$, the narrowest range among the units. With a few exceptions, the feldspars analyzed displayed little chemical variation among major elements. MS-15-7c contains the lowest concentrations of $\mathrm{Al}_{2} \mathrm{O}_{3}(\sim 19.68 \%), \mathrm{FeO}(\sim 0.19 \%)$ and $\mathrm{CaO}(\sim 0.58 \%)$ and the highest $\mathrm{K}_{2} \mathrm{O}(\sim 4.32 \%)$ concentrations of all the samples. No individual sample contained the highest or lowest elemental concentrations across the major elements. Compared to other units, unit 4 feldspars contain the highest concentrations of $\mathrm{SiO}_{2}$ $(\sim 68.52 \%), \mathrm{K}_{2} \mathrm{O}(\sim 4.32 \%)$ and $\mathrm{BaO}(\sim 1.04 \%)$. Concentrations of $\mathrm{FeO}(\sim 0.19 \%)$, $\mathrm{Al}_{2} \mathrm{O}_{3}(\sim 19.68 \%), \mathrm{Na}_{2} \mathrm{O}(\sim 7.53 \%)$ and $\mathrm{CaO}(\sim 0.58 \%)$ are the lowest in unit 4 , but the $\mathrm{FeO}^{*}$ and $\mathrm{Na}_{2} \mathrm{O}$ concentrations are comparable to the other units while the $\mathrm{CaO}$ concentrations are comparable only to unit 3. 
Table 6. Unit 4 average EMP major element feldspar chemical composition by sample.

\begin{tabular}{lccccccc}
\hline Sample & $\begin{array}{c}\text { Number } \\
\text { of } \\
\text { Crystals }\end{array}$ & $\begin{array}{c}\mathrm{SiO}_{2} \\
(\text { wt. \%) }\end{array}$ & $\begin{array}{c}\mathrm{SiO}_{2} \\
\text { Std. }\end{array}$ & $\begin{array}{c}\mathrm{Al}_{2} \mathbf{O}_{3} \\
(\text { wt. \%) }\end{array}$ & $\begin{array}{c}\mathrm{Al}_{2} \mathbf{O}_{3} \\
\text { Std }\end{array}$ & $\begin{array}{c}\mathrm{FeO}^{*} \\
(\text { wt. \%) }\end{array}$ & $\begin{array}{c}\mathrm{FeO}^{*} \\
\text { Std. }\end{array}$ \\
\hline MS-12-20 & 7 & 68.52 & 0.96 & 20.50 & 0.25 & 0.20 & 0.02 \\
MS-15-18-2T & 3 & 67.52 & 0.73 & 20.45 & 0.18 & 0.23 & 0.04 \\
MS-15-7c & 2 & 67.79 & 0.32 & 19.68 & 0.14 & 0.19 & 0.02 \\
DIT@Craft & 10 & 66.79 & 0.78 & 20.48 & 0.17 & 0.20 & 0.03 \\
Point & 10 & 66.96 & 0.51 & 20.48 & 0.32 & 0.23 & 0.04 \\
MS-15-20a & 10 & & & & &
\end{tabular}

Table 6 continued.

\begin{tabular}{lccccc}
\hline \hline Sample & $\begin{array}{c}\text { Number } \\
\text { of } \\
\text { Crystals }\end{array}$ & $\begin{array}{c}\mathbf{C a O} \\
(\text { wt. \%) }\end{array}$ & $\begin{array}{c}\mathrm{CaO} \\
\text { Std. }\end{array}$ & $\begin{array}{c}\mathrm{Na}_{2} \mathbf{O} \\
(\text { wt. \%) }\end{array}$ & $\begin{array}{c}\mathrm{Na}_{2} \mathbf{O} \\
\text { Std. }\end{array}$ \\
\hline MS-12-20 & 7 & 0.78 & 0.13 & 8.68 & 0.13 \\
MS-15-18-2T & 3 & 0.88 & 0.01 & 8.41 & 0.42 \\
MS-15-7c & 2 & 0.58 & 0.00 & 7.53 & 0.12 \\
DIT@Craft Point & 10 & 0.84 & 0.06 & 8.54 & 0.28 \\
MS-15-20a & 10 & 0.81 & 0.24 & 8.67 & 0.31 \\
\hline
\end{tabular}

Table 6 continued.

\begin{tabular}{lccccc}
\hline \hline Sample & $\begin{array}{c}\text { Number } \\
\text { of } \\
\text { Crystals }\end{array}$ & $\begin{array}{c}\mathbf{K}_{\mathbf{2}} \mathbf{O} \\
(\mathbf{w t .} \%)\end{array}$ & $\begin{array}{c}\mathbf{K}_{\mathbf{2}} \mathbf{O} \\
\text { Std. }\end{array}$ & $\begin{array}{c}\text { BaO } \\
(\mathbf{w t .} \%)\end{array}$ & $\begin{array}{c}\text { BaO } \\
\text { Std. }\end{array}$ \\
\hline MS-12-20 & 7 & 2.89 & 0.07 & 0.99 & 0.10 \\
MS-15-18-2T & 3 & 2.83 & 0.03 & N/A & N/A \\
MS-15-7c & 2 & 4.32 & 0.44 & N/A & N/A \\
DIT@Craft Point & 10 & 2.92 & 0.33 & 1.04 & 0.07 \\
MS-15-20a & 10 & 3.18 & 0.72 & 0.92 & 0.20 \\
\hline
\end{tabular}




\section{Extent Maps}

\section{Unit 1}

Figure 16 shows the locations of unit 1 designated samples and the overall extent of unit 1 . Unit 1 covers an area of $\sim 22,590 \mathrm{~km}^{2}$ and is the oldest (16.15-16 Ma) and most widespread of the four units in terms of areal extent, spreading farther eastward and westward than any of the units. GPS coordinates associated with the samples correlated to unit 1 in this study Appendix A.

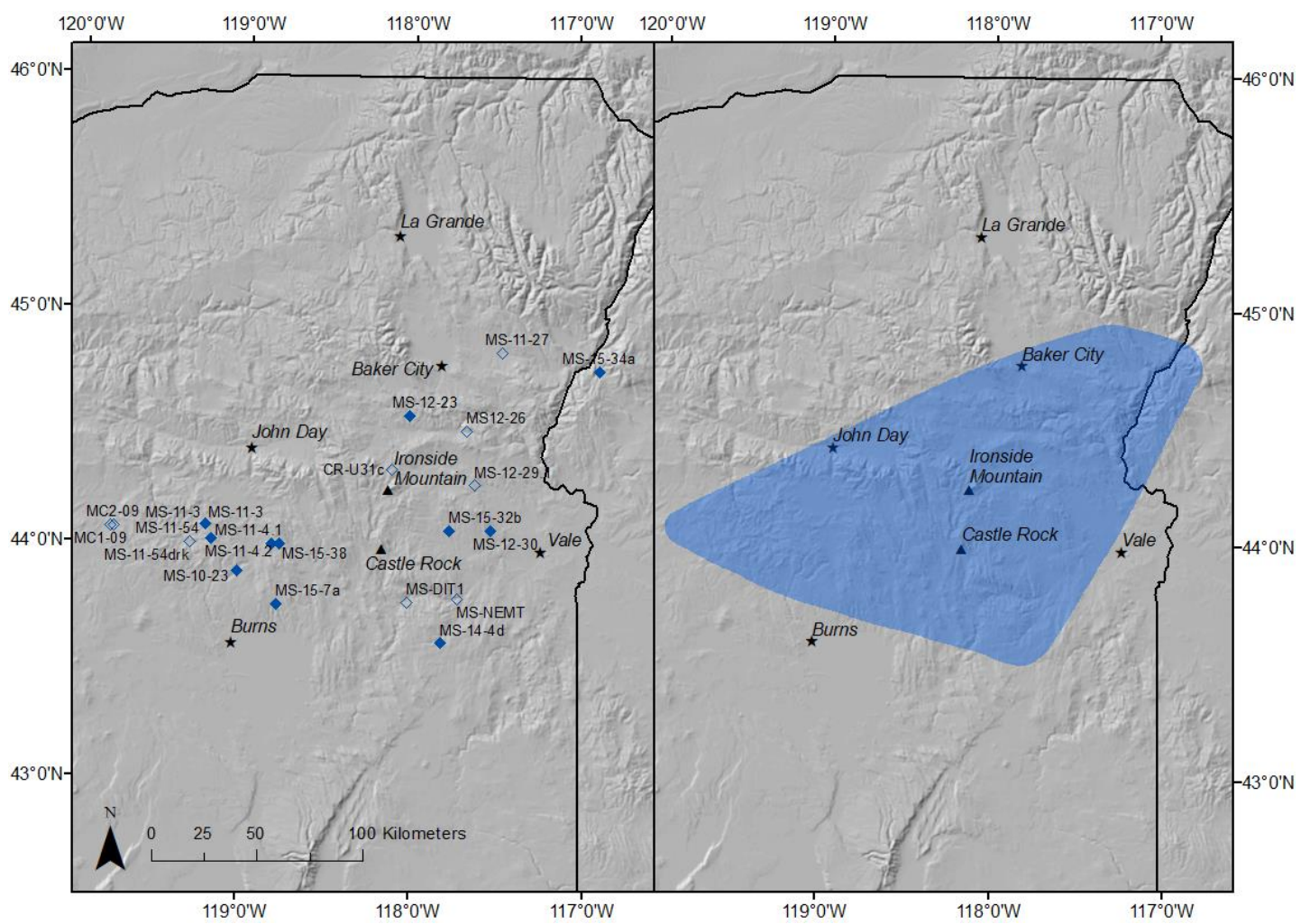

Figure 16. Areal extent of the Dinner Creek Tuff. Left: Sample names and locations utilized in determining the extent. Open symbols represent samples from Streck et al. (2015) while solid symbols represent samples from this study. Right: Overall areal extent of unit 1 covers an area of $\sim 22,590 \mathrm{~km}^{2}$. Castle Rock and Ironside Mountain are the eruption sites for units 1 and 2, respectively. They also mark the north/south boundaries of the DITEC. 


\section{Unit 2}

Figure 17 shows the locations of unit 2 designated samples and the overall extent of unit 1 . Unit 2 covers an area of $\sim 17,920 \mathrm{~km}^{2}$. Unit 2 is the second oldest (15.6-15.5 Ma) and second most widespread unit in terms of areal extent while extending further north than any of the units. GPS coordinates associated with the samples correlated to unit 2 in this study are found in Appendix B.

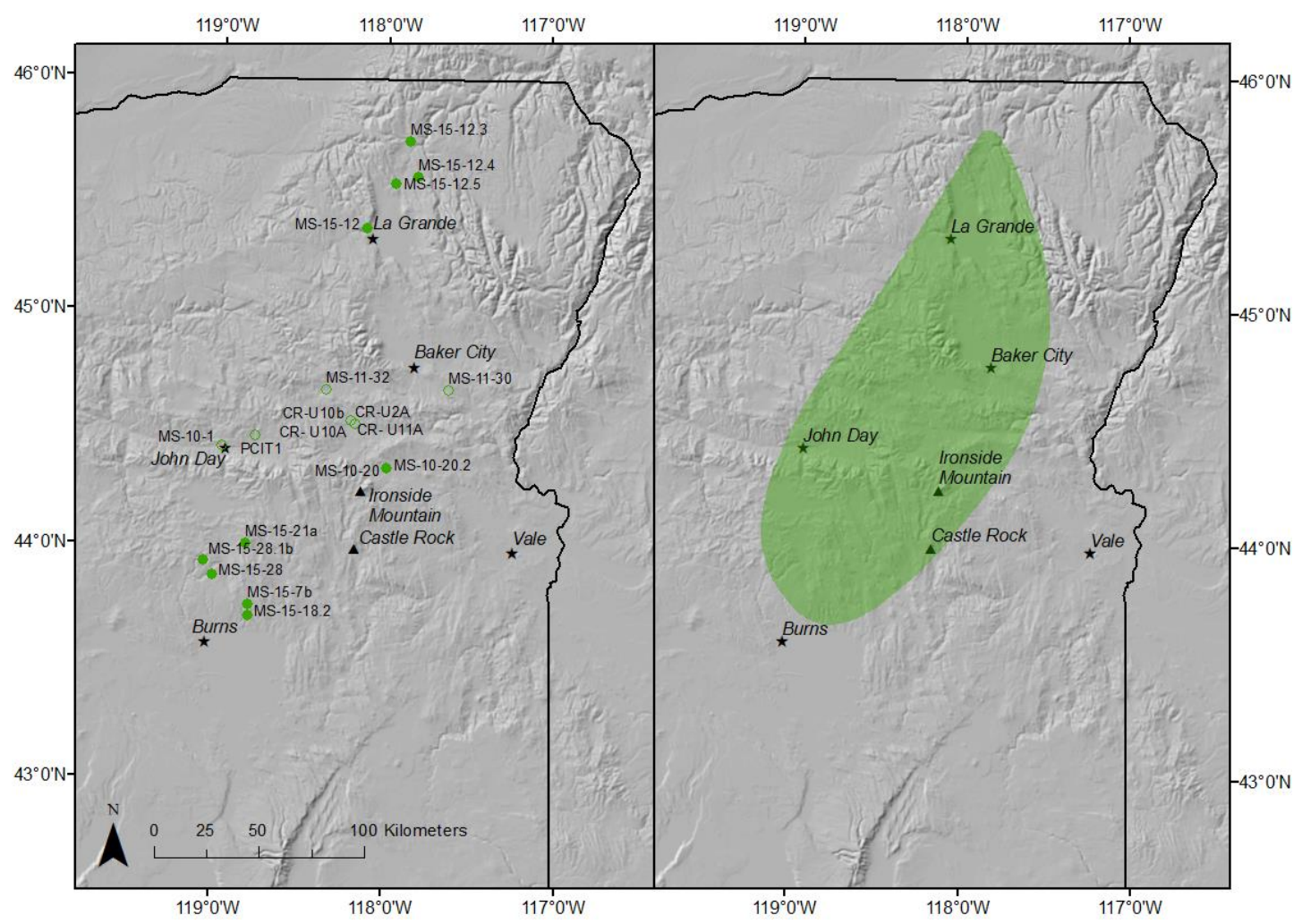

Figure 17. Areal extent of the Dinner Creek Tuff. Left: Sample names and locations utilized in determining the extent. Open symbols represent samples from Streck et al. (2015) while solid symbols represent samples from this study. Right: Overall areal extent of unit 2 covers an area of $\sim 17,920 \mathrm{~km}^{2}$. Castle Rock and Ironside Mountain are the eruption sites for units 1 and 2, respectively. They also mark the approximate north/south boundaries of the DITEC. 


\section{Unit 3}

Figure 18 shows the locations of unit 3 designated samples and the overall extent of unit 3. Unit 3 covers an area of $\sim 14,170 \mathrm{~km}^{2}$. The third youngest (15.46 Ma) and third smallest of the units in terms of areal extent while extending farther south than any of the units. GPS coordinates associated with the samples correlated to unit 3 in this study are found in Appendix C.

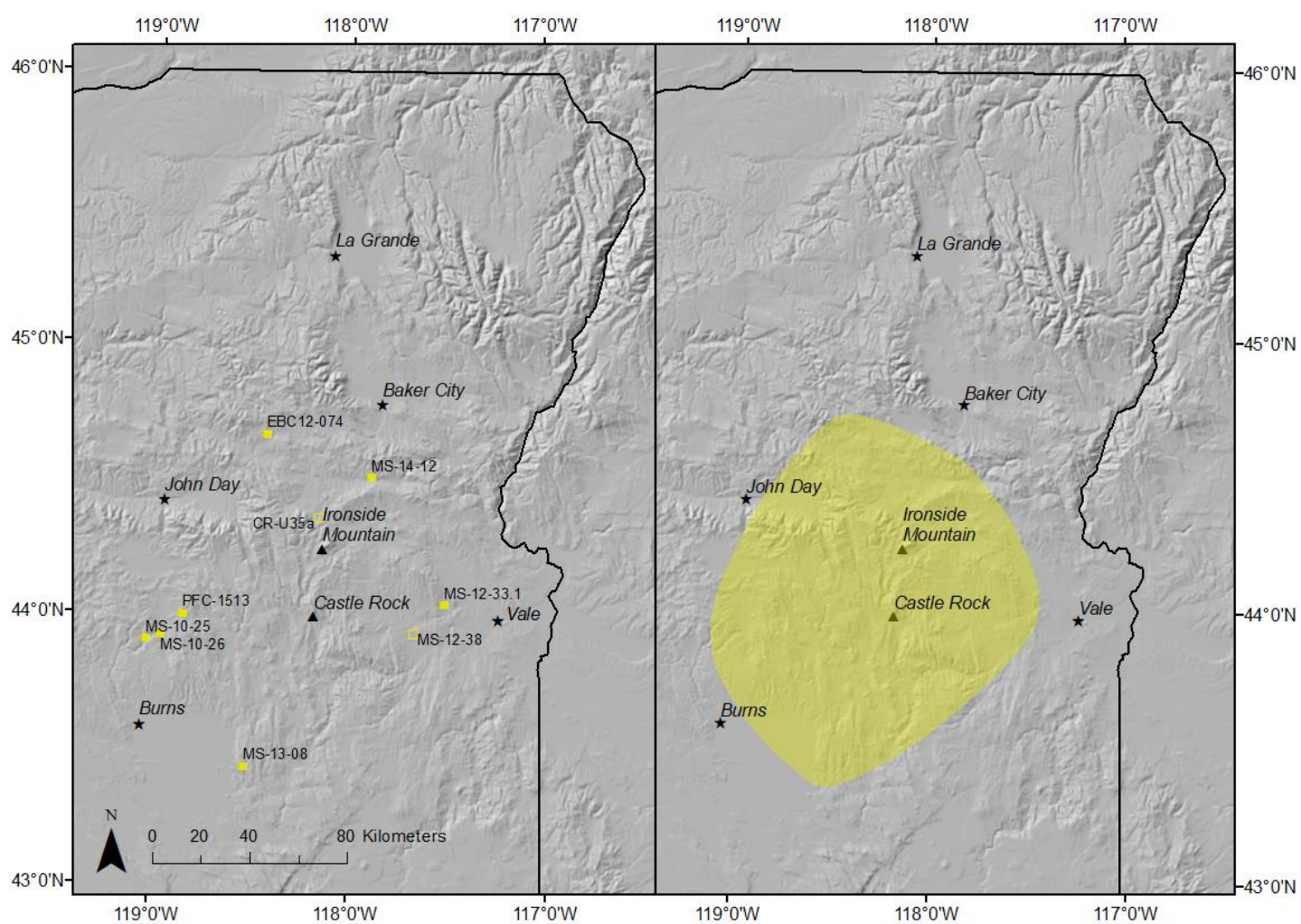

Figure 18. Areal extent of the Dinner Creek Tuff. Left: Sample names and locations utilized in determining the extent. Open symbols represent samples from Streck et al. (2015) while solid symbols represent samples from this study. Right: Overall areal extent of unit 3 covers an area of $\sim 14,170 \mathrm{~km}^{2}$. Castle Rock and Ironside Mountain are the eruption sites for units 1 and 2, respectively. They also mark the approximate north/south boundaries of the DITEC. 


\section{Unit 4}

Figure 19 shows the locations of unit 4 designated samples and the overall extent of unit 4 . Unit 4 covers an area of $\sim 8,370 \mathrm{~km}^{2}$, the smallest in terms of areal extent and the youngest (15.0 Ma) of the units. GPS coordinates associated with the samples correlated to unit 4 in this study are found in Appendix D.

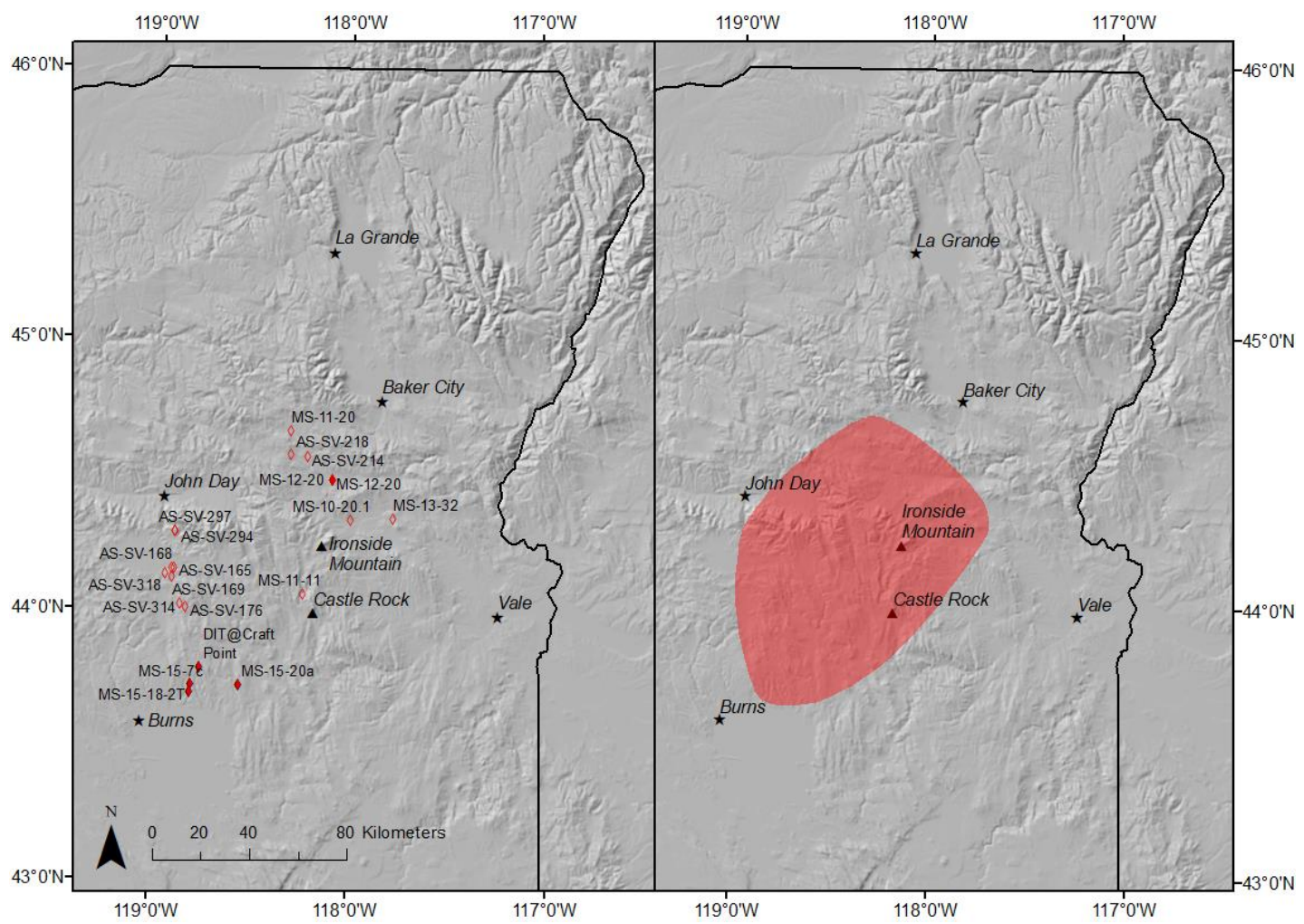

Figure 19. Areal extent of the Dinner Creek Tuff. Left: Sample names and locations utilized in determining the extent. Open symbols represent samples from Streck et al. (2015) and correlated samples collected by Aaron Steiner, while solid symbols represent samples from this study. Right: Overall areal extent of unit 4 covers an area of $\sim 8,370 \mathrm{~km}^{2}$. Castle Rock and Ironside Mountain are the eruption sites for units 1 and 2, respectively. They also mark the approximate north/south boundaries of the DITEC. 


\section{Total extent}

Overall, the DIT areal extent covers an area of $\sim 31,800 \mathrm{~km}^{2}$ (Figure 20).

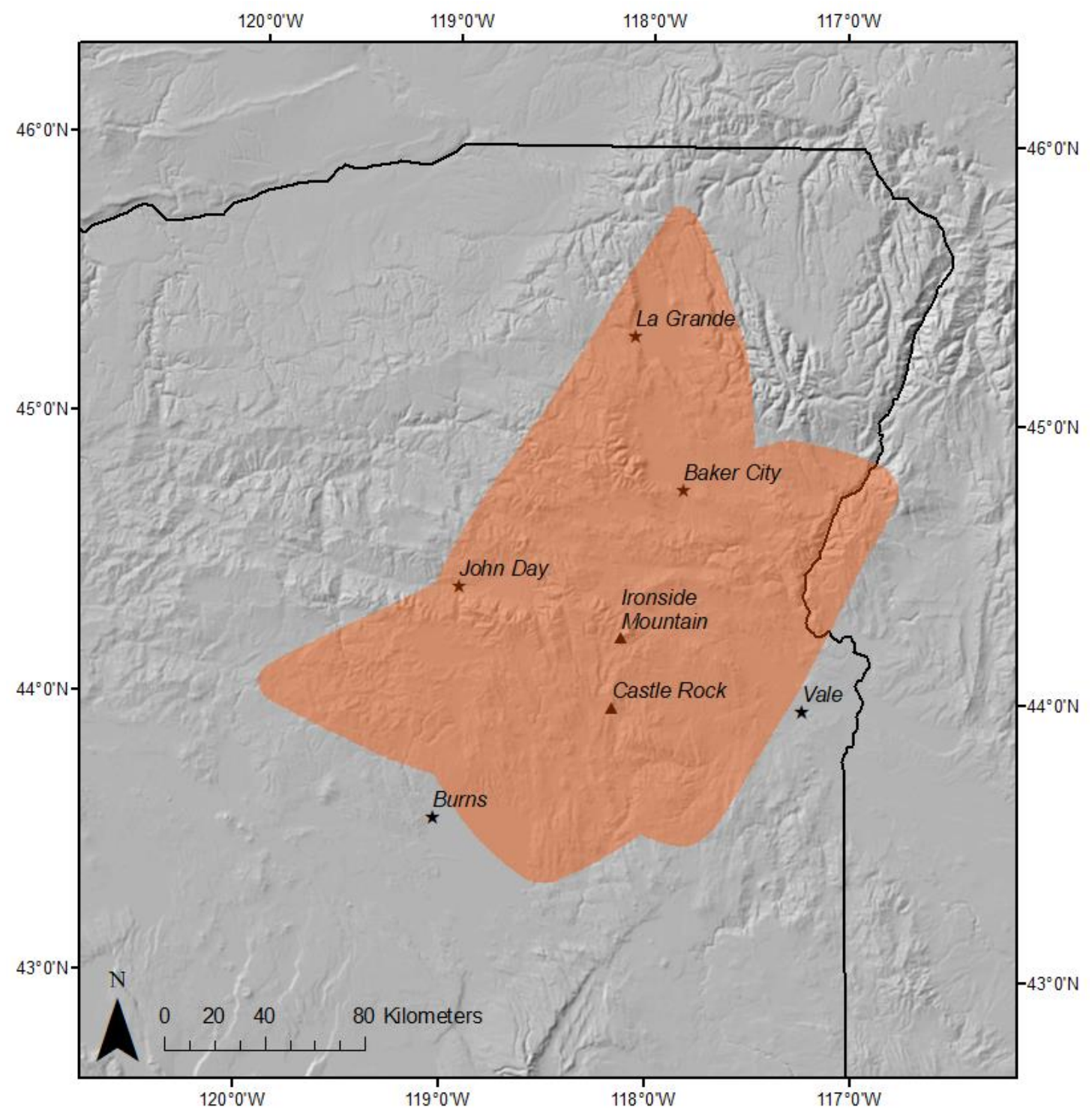

Figure 20. Overall areal extent of the Dinner Creek Tuff covers an area of $\sim 31,800 \mathrm{~km}^{2}$. Castle Rock and Ironside Mountain are the eruption sites for units 1 and 2, respectively. They also mark the approximate north/south boundaries of the DITEC. 


\section{Non-unit Designating Feldspars}

Samples from every unit contained non-unit designating feldspars, which are feldspars with anorthite and orthoclase concentrations that lie outside the concentrations used to correlate felspars to an individual cooling unit: unit 1 ( $\mathrm{An}_{10-15}$, Or6-10), unit 2 (An $\left.\mathrm{An}_{19-23}, \mathrm{Or}_{4-6}\right)$ and units 3/4 (An $\left.3-6, \mathrm{Or}_{16-27}\right)$. Non-unit designating feldspar types include: albite, andesine, anorthoclase, bytownite, labradorite, oligoclase and sanidine (Figures 21-25). Unit 2 contains 35 non-unit designating feldspar crystals from 8 samples, the most of any unit followed by unit 4 with 12 crystals from 3 samples. Unit 3 contains 6 non-unit designating feldspar crystals from 2 samples, while unit 1 has the fewest with 3 crystals from 2 samples. Appendix E contains associated individual crystal chemical data and end member concentrations. 


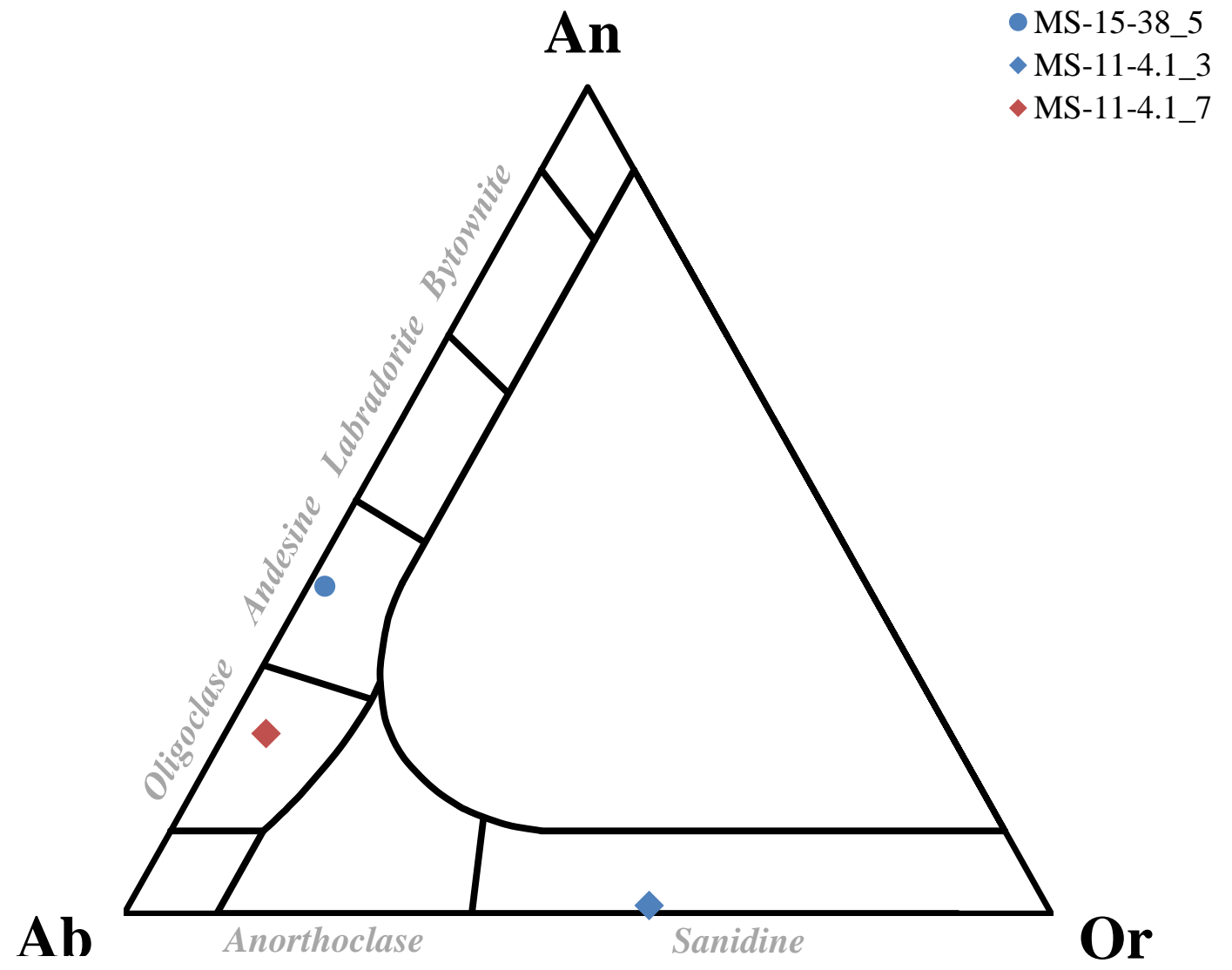

Figure 21. Non-unit designating feldspar endmember concentration diagram for samples associated with unit 1. 


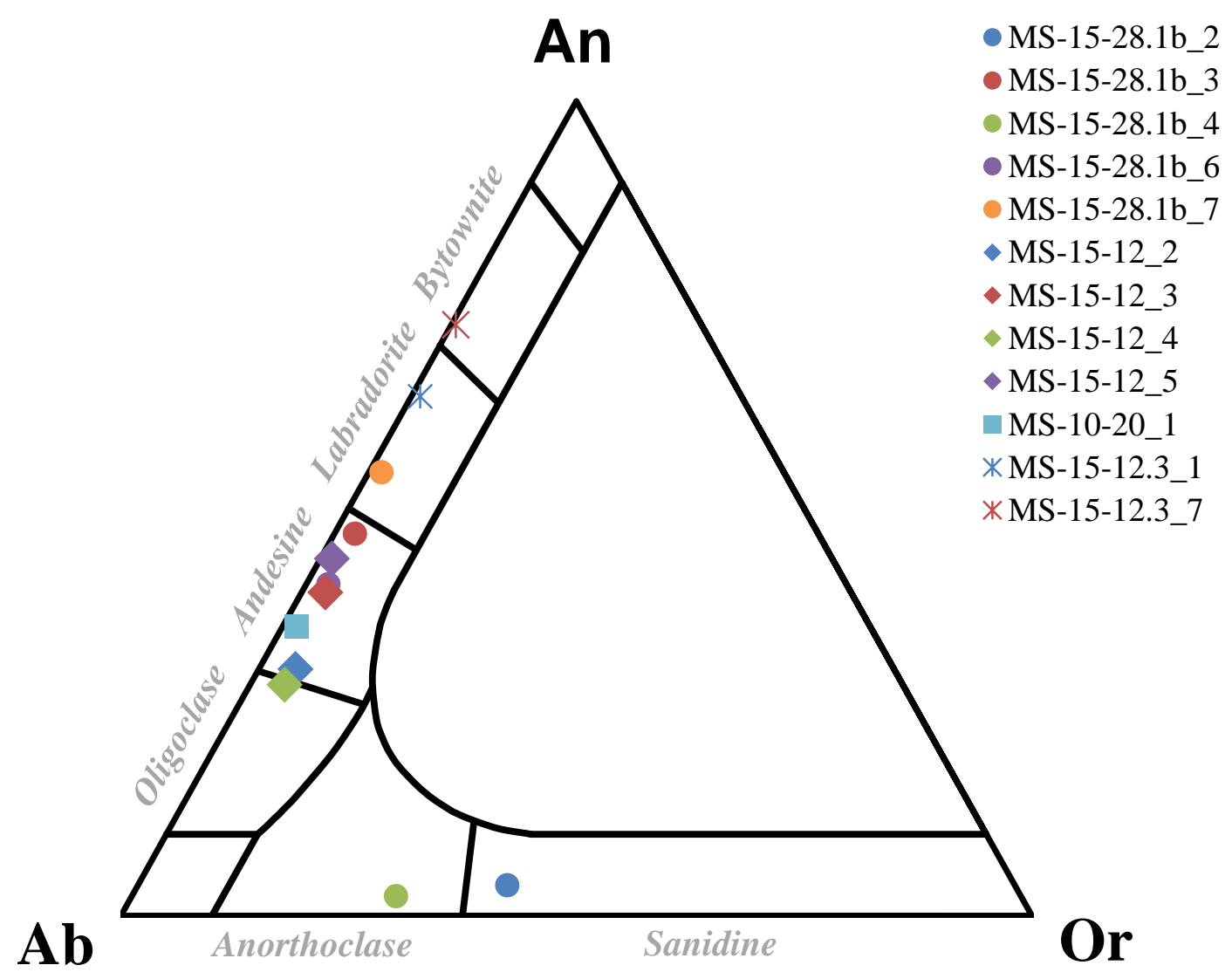

Figure 22a. Non-unit designating feldspar endmember concentration diagram for samples associated with unit 2. 


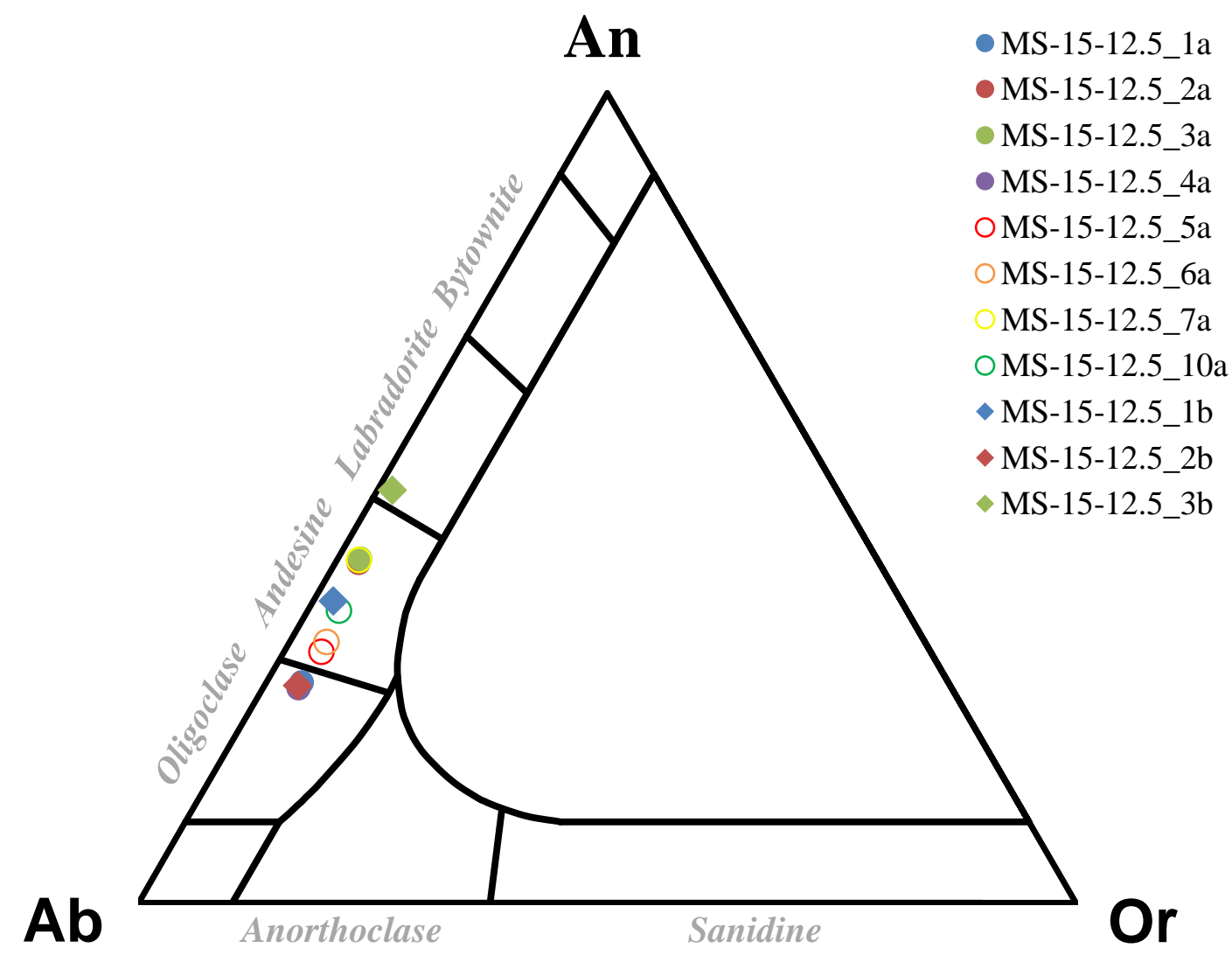

Figure 22b. Non-unit designating feldspar endmember concentration diagram for samples associated with unit 2. 


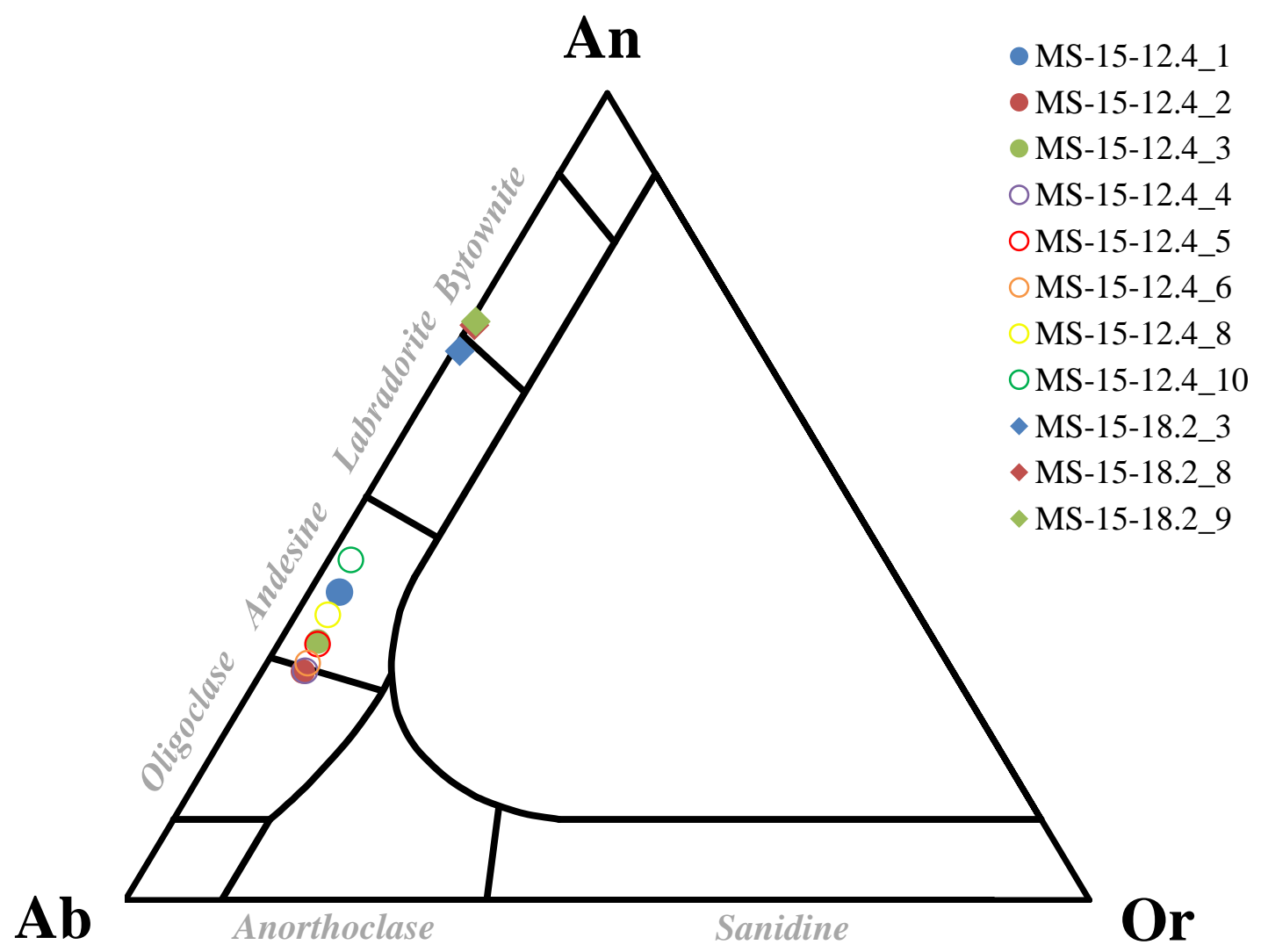

Figure 22c. Non-unit designating feldspar endmember concentration diagram for samples associated with unit 2. 


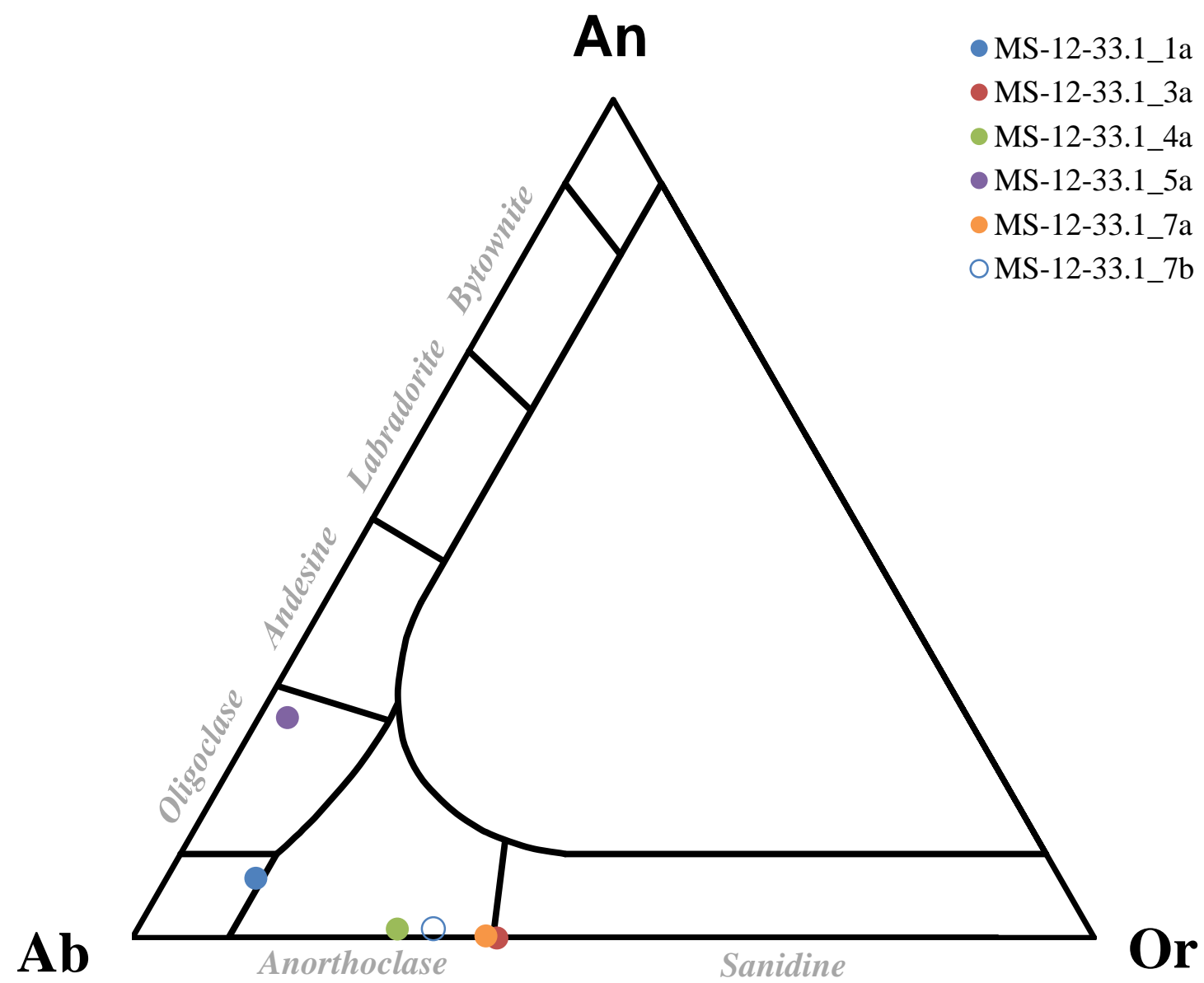

Figure 23. Non-unit designating feldspar endmember concentration diagram for samples associated with unit 3. 


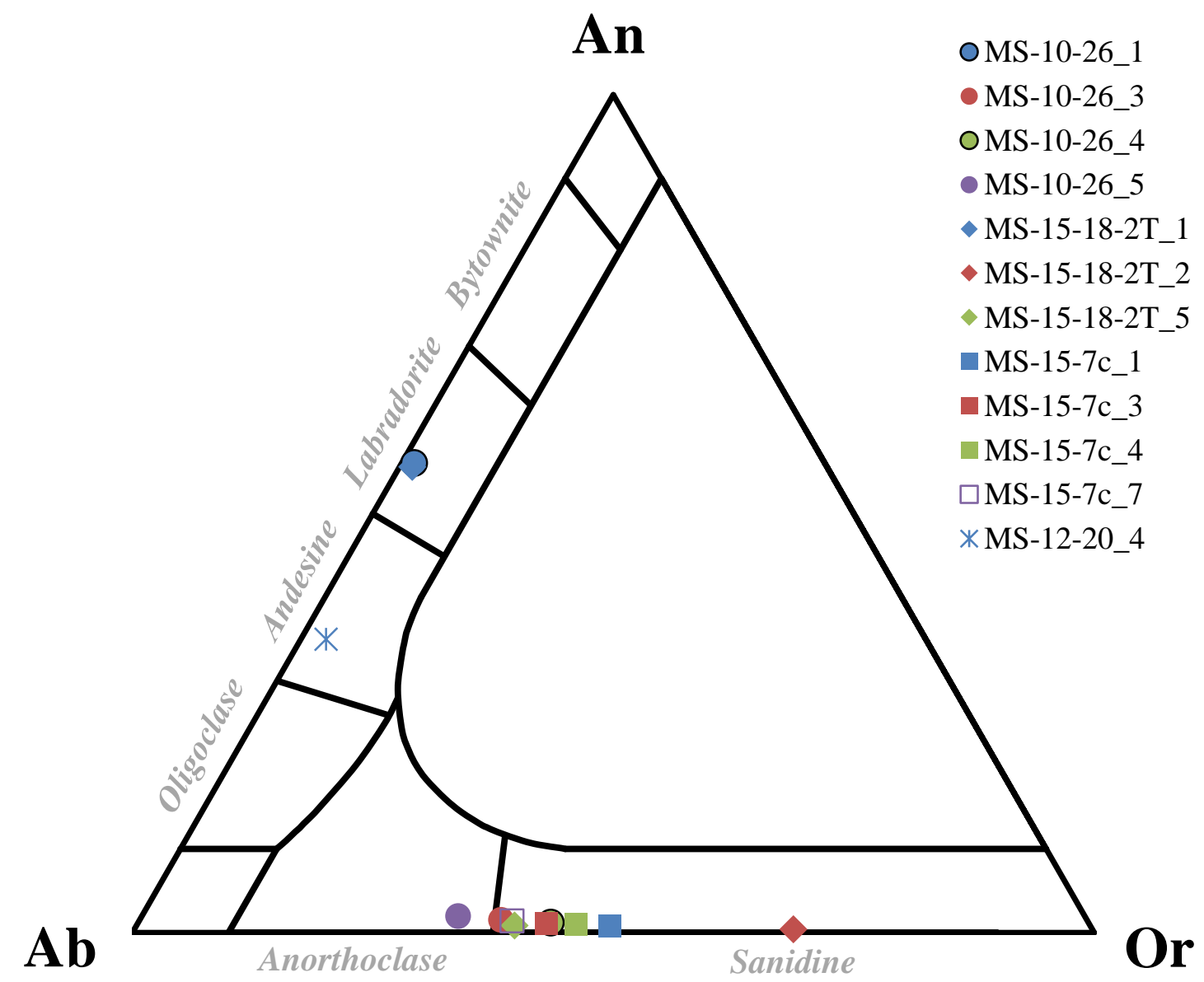

Figure 24. Non-unit designating feldspar endmember concentration diagram for samples associated with unit 4. 


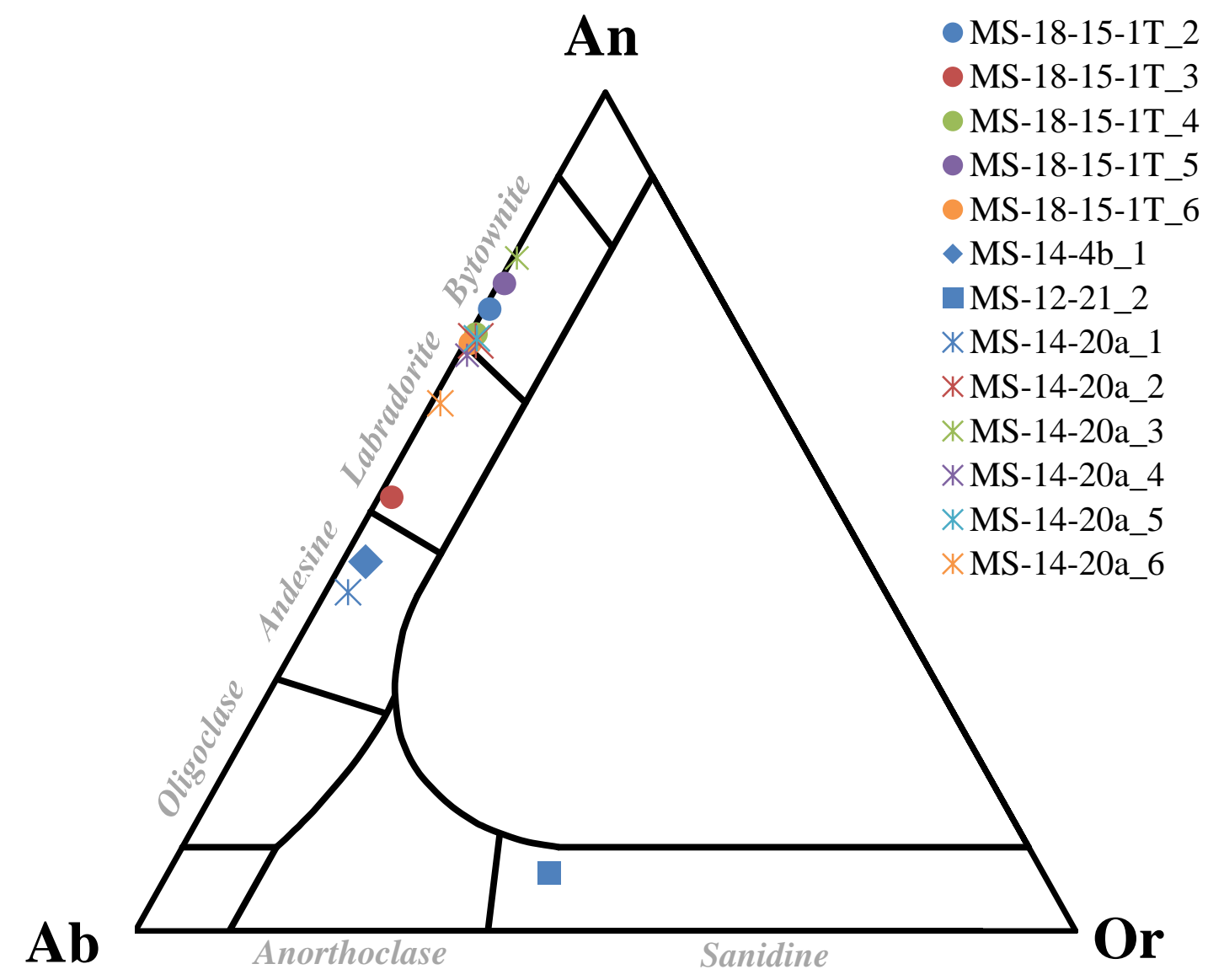

Figure 25. Non-unit designating feldspar endmember concentration diagram for samples not correlated to a unit. 


\section{DISCUSSION}

\section{Erupted Tuff Volumes}

Total volume $\left(V_{t}\right)$ for each eruptive unit was calculated using two individual cylinder volumes (large: $V_{L}$ and small: $V_{S}$ ) to approximate thinning of the tuff away from the source:

$$
V_{t}=V_{L}+V_{S}
$$

Equation 1

The large cylinder volumes $\left(V_{L}\right)$ were calculated by treating the unit areal extent as the area $(A)$ of a circle and using conservative thicknesses $(h)$ (Table 7). Thicknesses used are averages based on observed thicknesses from Kittlemen, et al. (1965), Haddock (1967), Davenport (1970) and Streck et al. (2015). Table 8 contains outcrop thicknesses observed during sample collection (Streck, pers. comm., 2017):

$$
V_{L}=A(h)
$$

Equation 2

The radius used to calculate the small circle area was half the radius of the large circle:

$$
\begin{aligned}
& r_{L}=\sqrt{\frac{A}{\pi}} \\
& r_{S}=0.5 r_{L} .
\end{aligned}
$$

Equation 3

Equation 4

The resulting small circle radius and thicknesses in Table 7 were used to calculate small circle volumes utilizing Equation 2. Determined tuff volumes are $\sim 170 \mathrm{~km}^{3}$ (unit 1), 125 km (unit 2), $\sim 99 \mathrm{~km}^{3}$ (unit 3) and $\sim 46 \mathrm{~km}^{3}$ (unit 4), totaling $\sim 440 \mathrm{~km}^{3}$. 
Table 7. Unit thicknesses used in volume calculations.

\begin{tabular}{lcccc}
\hline \hline Circle & $\begin{array}{c}\text { Unit 1 } \\
(\mathbf{m})\end{array}$ & $\begin{array}{c}\text { Unit 2 } \\
(\mathbf{m})\end{array}$ & $\begin{array}{c}\text { Unit 3 } \\
(\mathbf{m})\end{array}$ & $\begin{array}{c}\text { Unit 4 } \\
(\mathbf{m})\end{array}$ \\
\hline Large & 5 & 5 & 5 & 4 \\
Small & 10 & 8 & 8 & 6 \\
\hline
\end{tabular}

Table 8. Observed thicknesses from distal outcrops of sample sites or sites proximal to sample location.

\begin{tabular}{lclc}
\hline \hline Sample & $\begin{array}{c}\text { Thickness } \\
(\mathbf{m})\end{array}$ & Sample & $\begin{array}{c}\text { Thickness } \\
(\mathbf{m})\end{array}$ \\
\cline { 1 - 1 } Unit 1 & & Unit 3 & \\
\cline { 1 - 1 } MC1-09 & 12 & MS-12-33.1 & 15 \\
MS-15-34a & 3 & MS-12-38 & 10 \\
MS-11-27 & 4 & & \\
Unit 2 & & Unit 4 & \\
\cline { 1 - 1 } MS-10-1 & $15-20$ & AS-SV-214 & 8 \\
PCIT1 & 12 & MS-15-2T & 10 \\
MS-15-7b & 5 & MS-15-20a & 7 \\
\hline
\end{tabular}

\section{Dense Rock Equivalent}

DRE calculates magma volume utilizing individual unit densities $\left(\rho_{t}\right)$, dense rock density $\left(\rho_{d r}=2.34 \mathrm{~g} / \mathrm{cm}^{3}\right)$ and erupted tuff volume for each unit $(V)$ calculated in this study (Streck and Grunder, 1995; Streck, et al., 2015; Streck pers. comm., 2017). Like erupted tuff volumes, DREs decreases with unit size and age (Table 9).

Table 9. Unit densities and DRE.

\begin{tabular}{lrcrc}
\hline \hline & $\begin{array}{r}\text { Unit 1 } \\
\left(\mathbf{g} / \mathbf{c m}^{3}\right)\end{array}$ & $\begin{array}{c}\text { Unit 2 } \\
\left(\mathbf{g} / \mathbf{c m}^{3}\right)\end{array}$ & $\begin{array}{r}\text { Unit 3 } \\
\left(\mathbf{g} / \mathbf{c m}^{3}\right)\end{array}$ & $\begin{array}{r}\text { Unit 4 } \\
\left(\mathbf{g} / \mathbf{c m}^{3}\right)\end{array}$ \\
\hline Unit density $\left(\mathrm{g} / \mathrm{cm}^{3}\right)$ & 2.1 & 1.8 & 1.8 & 1.6 \\
$\mathrm{DRE}^{*}\left(\mathrm{~km}^{3}\right)$ & 152.1 & 96.5 & 76.3 & 31.5 \\
\hline$*_{D R E}=\frac{\rho_{t}}{\rho_{d r}}(V)$ & & & & \\
\hline
\end{tabular}




\section{Probable Extents}

Areal extents were determined by enclosing sample locations within a polygon. The polygon also included the DITEC, but not in a centralized location. These factors lead to irregular shaped extents inconsistent with low-aspect ratio, high energy ignimbrites (LAI). Aspect ratio is determined by dividing the average thickness by the horizontal dimension of an ignimbrite (Walker, 1983). The response of an ash-flow to topography varies with aspect ratio. LAIs radiate outwards from the eruptive site, tending to travel a uniform distance in all directions, overtopping hills while blanketing the landscape rather than passively flowing through existing valleys (Walker, 1983; Freundt and Rosi, 1998; Sigurdsson et al., 2015). Based on the expertise and experience Martin Streck has accumulated during his extensive studies of eastern Oregon volcanism, the author of this study created probable areal extents for units 1-3 that are more in line with LIAs, centering the source areas within the polygons (Figures 26-29). Since the source for unit 3 is unknown and the DITEC is already located near the center of the extent area, only minimal adjustments were needed. An increase in areal extent also increases the eruptive volumes and DREs. Table 10 summarizes extent sizes, erupted tuff volumes and DREs associated with the determined and probable extents. 
Table 10. Approximate determined and probable extent areal extents, volumes and DREs.

\begin{tabular}{lcccccc}
\hline \hline Unit & $\begin{array}{c}\text { Determined } \\
\text { Area } \\
\left(\mathrm{km}^{2}\right)\end{array}$ & $\begin{array}{c}\text { Probable } \\
\text { Area } \\
\left(\mathrm{km}^{2}\right)\end{array}$ & $\begin{array}{c}\text { Determined } \\
\text { Volume } \\
\left(\mathrm{km}^{3}\right)\end{array}$ & $\begin{array}{c}\text { Probable } \\
\text { Volume } \\
\left(\mathrm{km}^{3}\right)\end{array}$ & $\begin{array}{c}\text { Determined } \\
\text { DRE } \\
\left(\mathrm{km}^{3}\right)\end{array}$ & $\begin{array}{c}\text { Probable } \\
\text { DRE } \\
\left(\mathrm{km}^{3}\right)\end{array}$ \\
\hline 1 & 22,590 & 36,900 & 169.5 & 276.8 & 152.1 & 248.4 \\
2 & 17,920 & 31,660 & 125.4 & 221.6 & 96.5 & 170.5 \\
3 & 14,170 & 17,290 & 99.1 & 121.0 & 76.3 & 93.1 \\
4 & 8,370 & 10,150 & 46.0 & 55.8 & 31.5 & 38.1 \\
\hline Total & 31,800 & 43,490 & N/A & N/A & N/A & N/A \\
Extent & \multirow{2}{*}{ N/A } & & & & &
\end{tabular}

\section{Unit 1}

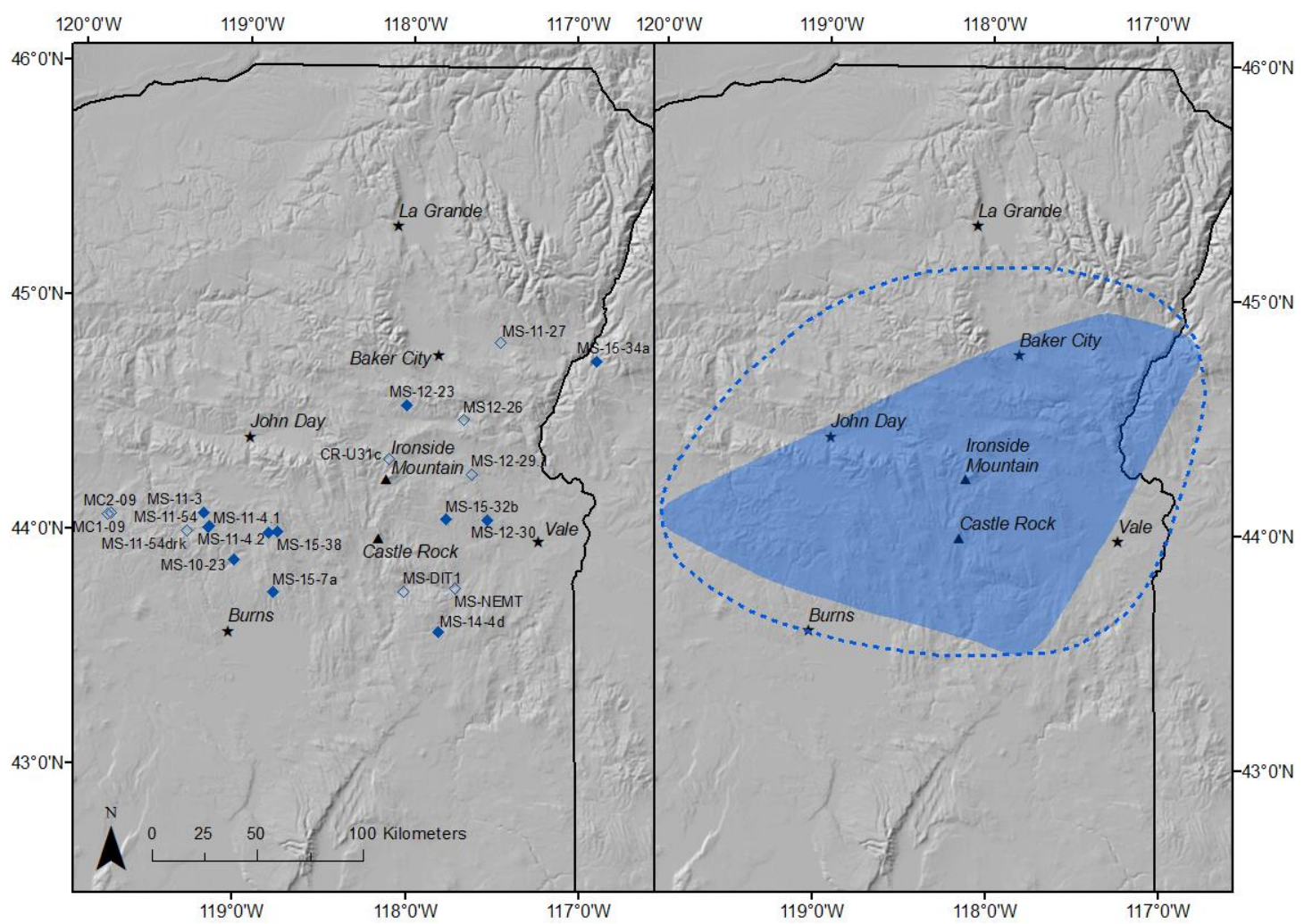

Figure 26. Probable areal extent of the Dinner Creek Tuff unit 1. Left: Sample names and locations utilized in determining the extent. Open symbols represent samples from Streck et al. (2015) while solid symbols represent samples from this study. Right: Solid shape - overall areal extent of unit 1 covers an area of $\sim 22,590 \mathrm{~km}^{2}$. Dashed line - probable extent covering an area of $\sim 36,900 \mathrm{~km}^{2}$. Castle Rock and Ironside Mountain are the eruption sites for units 1 and 2, respectively. They also mark the approximate north/south boundaries of the DITEC. 


\section{Unit 2}

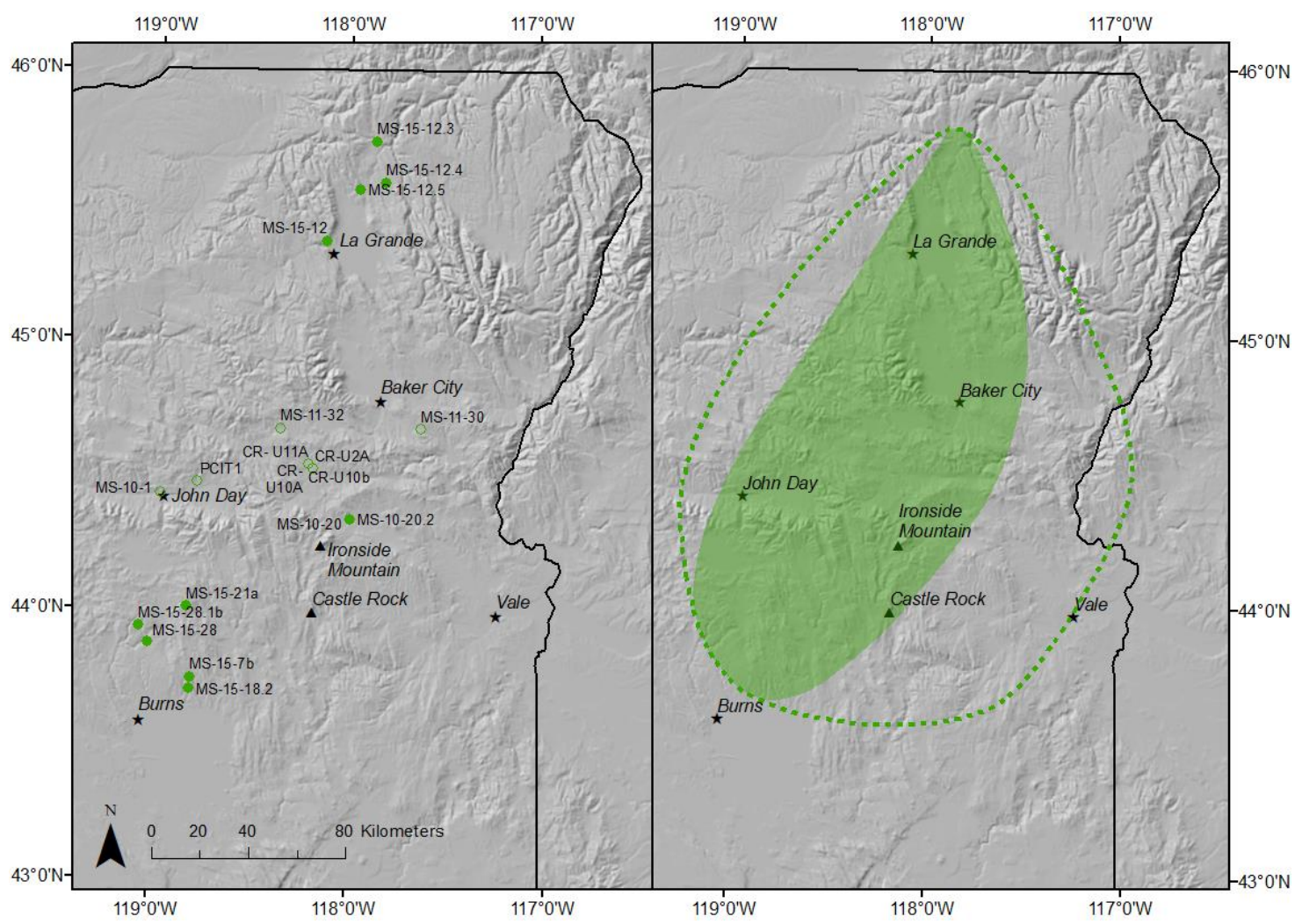

Figure 27. Probable areal extent of the Dinner Creek Tuff unit 2. Left: Sample names and locations utilized in determining the extent. Open symbols represent samples from Streck et al. (2015) while solid symbols represent samples from this study. Right: Solid shape - overall areal extent of unit 2 covers an area of $\sim 17,920 \mathrm{~km}^{2}$. Dashed line-probable extent covering an area of $\sim 31,660 \mathrm{~km}^{2}$. Castle Rock and Ironside Mountain are the eruption sites for units 1 and 2, respectively. They also mark the approximate north/south boundaries of the DITEC. 


\section{Unit 3}

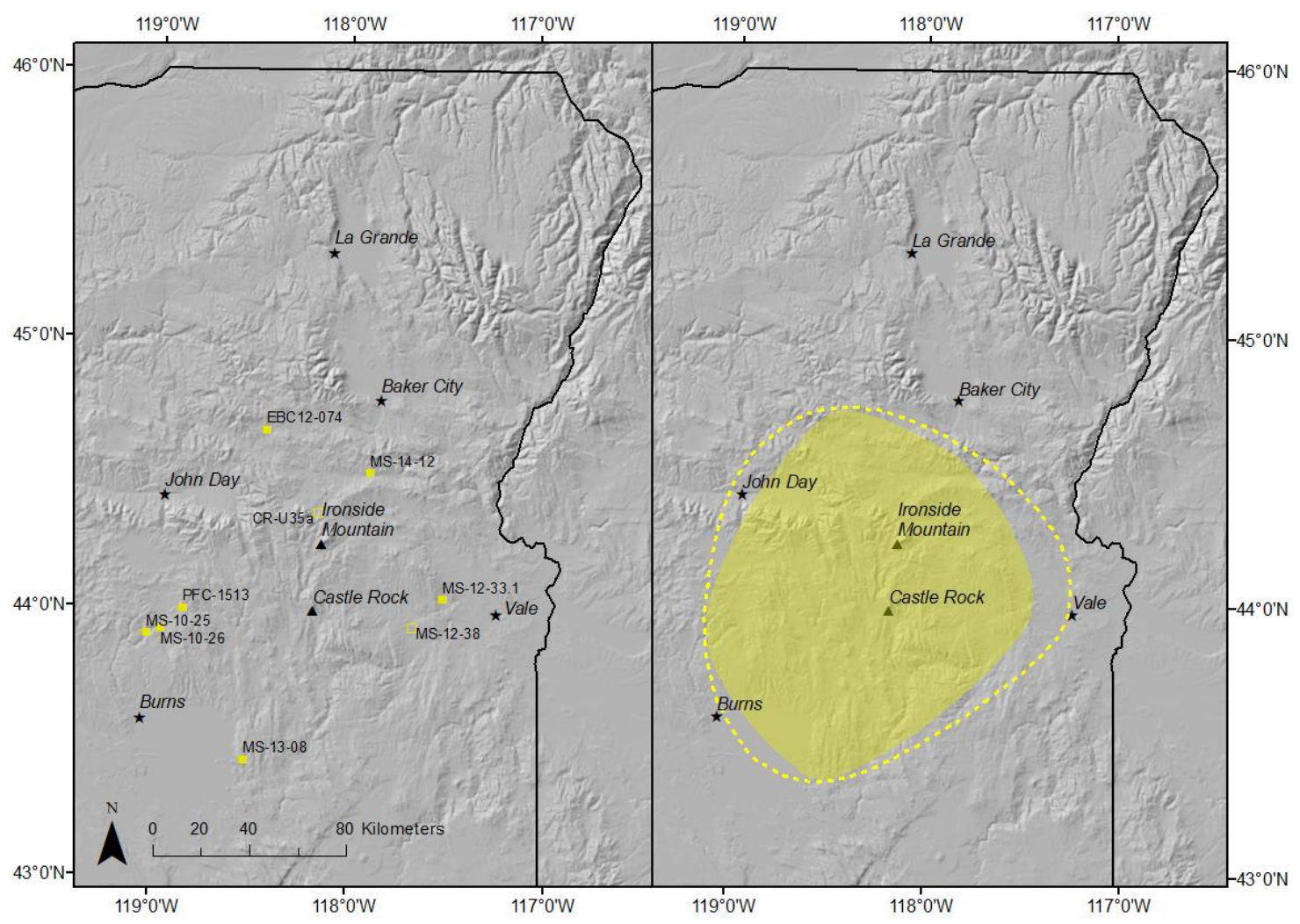

Figure 28. Probable areal extent of the Dinner Creek Tuff unit 3. Left: Sample names and locations utilized in determining the extent. Open symbols represent samples from Streck et al. (2015) while solid symbols represent samples from this study. Right: Solid shape - overall areal extent of unit 3 covers an area of $\sim 14,170 \mathrm{~km}^{2}$. Dashed line-probable extent covering an area of $\sim 17,290 \mathrm{~km}^{2}$. Castle Rock and Ironside Mountain are the eruption sites for units 1 and 2, respectively. They also mark the approximate north/south boundaries of the DITEC. 


\section{Unit 4}

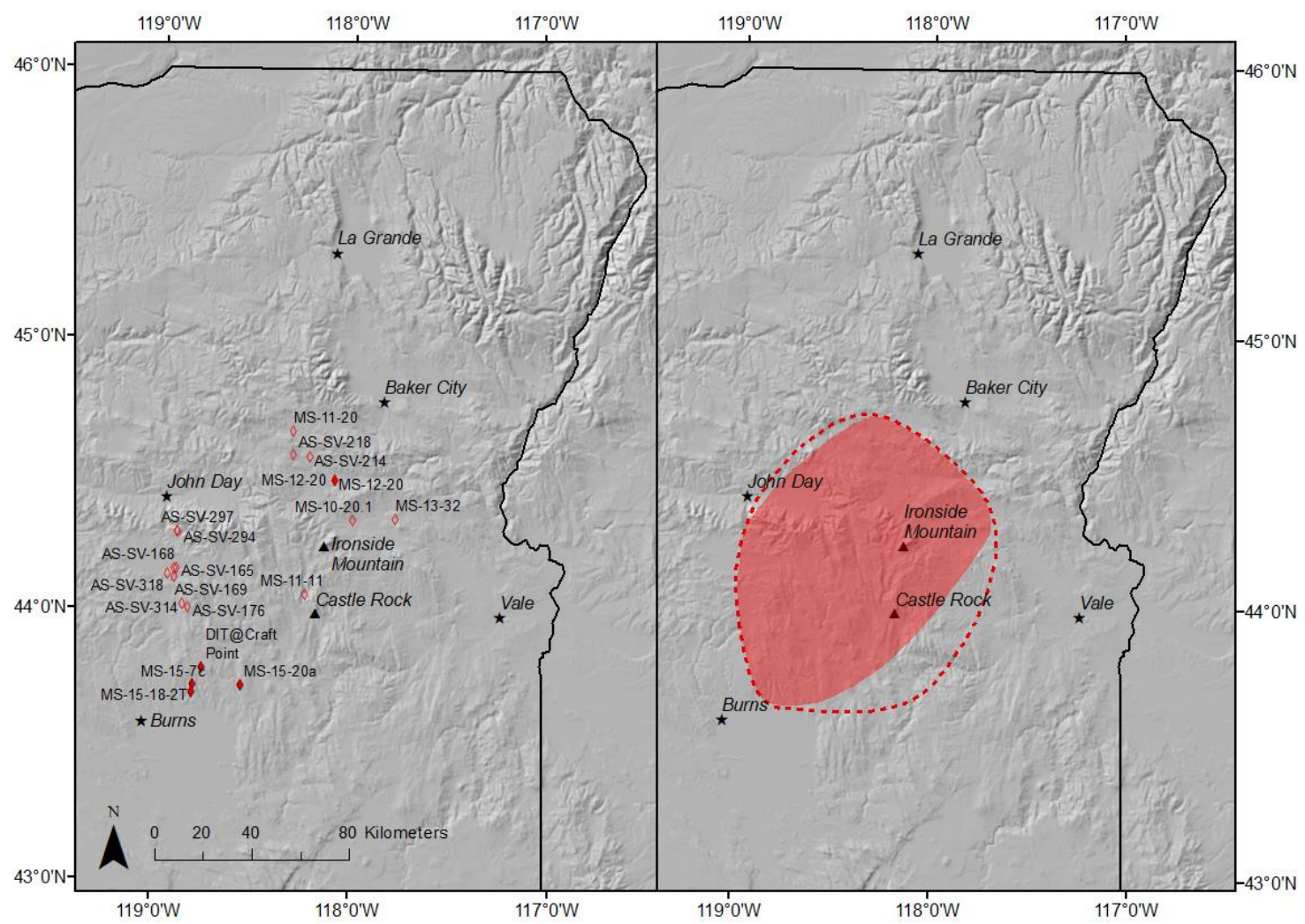

Figure 29. Probable areal extent of the Dinner Creek Tuff unit 4. Left: Sample names and locations utilized in determining the extent. Open symbols represent samples from Streck et al. (2015) and correlated samples collected by Aaron Steiner while solid symbols represent samples from this study. Right: Solid shape - overall areal extent of unit 4 covers an area of $\sim 8,370 \mathrm{~km}^{2}$. Dashed line-probable extent covering an area of $\sim 10,150 \mathrm{~km}^{2}$. Castle Rock and Ironside Mountain are the eruption sites for units 1 and 2, respectively. They also mark the approximate north/south boundaries of the DITEC. 


\section{Total Extent}

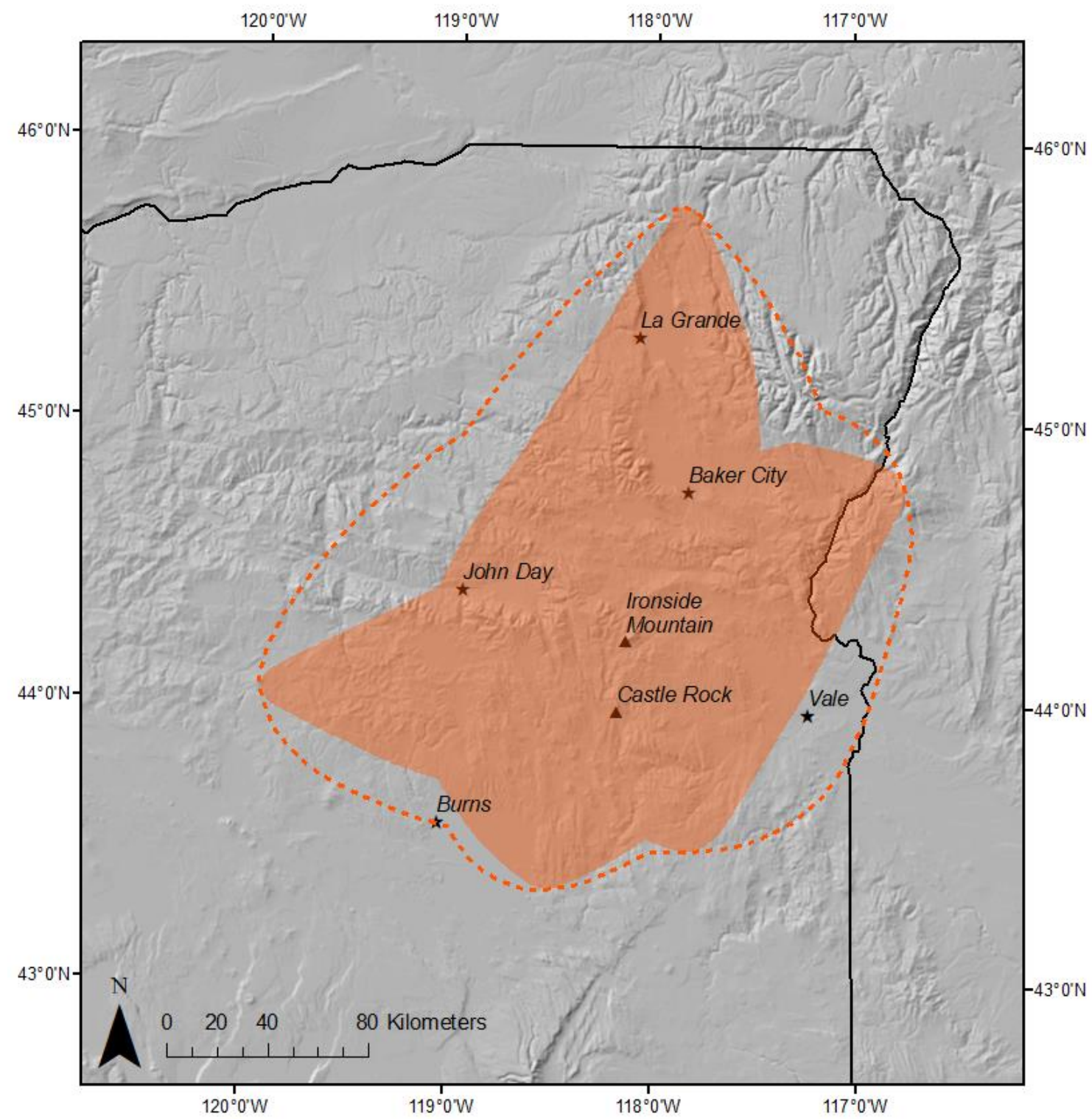

Figure 30. Solid shape-Overall areal extent of the Dinner Creek Tuff covers an area of $\sim 31,800 \mathrm{~km}^{2}$. Dashed line - overall probable areal extent covers an area of $\sim 43,490 \mathrm{~km}^{2}$. Castle Rock and Ironside Mountain are the eruption sites for units 1 and 2, respectively. They also mark the approximate north/south boundaries of the DITEC. 


\section{Erupted tuff volumes and caldera deposits}

Determined tuff volumes are $\sim 170 \mathrm{~km}^{3}$ (unit 1), $125 \mathrm{~km}^{3}$ (unit 2), $\sim 99 \mathrm{~km}^{3}$ (unit 3) and $\sim 46 \mathrm{~km}^{3}$ (unit 4), totaling $\sim 440 \mathrm{~km}^{3}$. The determined volumes calculated did not take into consideration the thicker caldera deposits. Cruz (pers. comm., 2017) calculated the minimum caldera volumes of units 1 and 2 to be $\sim 98.5 \mathrm{~km}^{3}$ and $\sim 31.1$ $\mathrm{km}^{3}$, respectively from the observations in Table 11 . In these calculations, Cruz assumes that the calderas collapsed along vertical or near vertical faults but suggests this is probably inaccurate given caldera collapse models and observations in more eroded calderas. He also assumes that the caldera floor collapsed as a cohesive block. Cruz acknowledges that the true tuff thicknesses should be much greater than the observed tuff thicknesses since at other similar sized calderas, the intra-caldera tuff has been found to be between 1-2 km thick. Based on a thickness of $1 \mathrm{~km}$, the volume of the intra caldera tuff for unit 1 at Castle Rock is $\sim 328.3 \mathrm{~km}^{3}$ and $\sim 51.8 \mathrm{~km}^{3}$ for unit 2 at Ironside Mountain. The densely welded nature of the caldera deposits means that the erupted tuff volume is equivalent to the DRE. Table 12 contains determined/probable volumes and DREs as well as volumes and DREs including intra caldera tuff volumes.

Table 11. Caldera observed thicknesses and axis used in minimal volume calculations*.

\begin{tabular}{lcc}
\hline \hline $\begin{array}{l}\text { Observations } \\
(\mathbf{k m})\end{array}$ & $\begin{array}{c}\text { Castle Rock } \\
\text { Caldera }\end{array}$ & $\begin{array}{c}\text { Ironside } \\
\text { Caldera }\end{array}$ \\
\hline Thickness $(t)$ & 0.3 & 0.6 \\
Long axis radius $(l)$ & 11.0 & 5.5 \\
Short axis radius $(s)$ & 9.5 & 3.0 \\
\hline$* V=t l s \pi$ & & \\
\hline
\end{tabular}


Table 12. Approximate determined and probable volumes and DREs including caldera deposits.

\begin{tabular}{|c|c|c|c|c|}
\hline Unit & $\begin{array}{l}\text { Determined } \\
\text { volume } \\
\left(\mathbf{k m}^{3}\right) \\
\end{array}$ & $\begin{array}{c}\text { Probable } \\
\text { volume } \\
\left(\mathbf{k m}^{3}\right) \\
\end{array}$ & $\begin{array}{c}\text { Determined volume } \\
\text { with caldera volume } \\
\left(\mathbf{k m}^{3}\right)\end{array}$ & $\begin{array}{c}\text { Probable volume } \\
\text { with caldera volume } \\
\left(\mathbf{k m}^{3}\right)\end{array}$ \\
\hline 1 & 169.5 & 276.8 & 268.0 & 375.3 \\
\hline 2 & 125.5 & 221.6 & 156.6 & 252.7 \\
\hline Unit & $\begin{array}{c}\text { Determined } \\
\text { DRE } \\
\left(\mathrm{km}^{3}\right) \\
\end{array}$ & $\begin{array}{c}\text { Probable } \\
\text { DRE } \\
\left(\mathrm{km}^{3}\right) \\
\end{array}$ & $\begin{array}{c}\text { Determined DRE with } \\
\text { caldera DRE } \\
\left(\mathrm{km}^{3}\right) \\
\end{array}$ & $\begin{array}{c}\text { Probable DRE with } \\
\text { caldera DRE } \\
\left(\mathrm{km}^{3}\right) \\
\end{array}$ \\
\hline 1 & 152.1 & 248.4 & 250.6 & 346.9 \\
\hline 2 & 96.5 & 170.5 & 127.6 & 201.6 \\
\hline
\end{tabular}

\section{Samples with multiple feldspar compositions}

Samples MS-11-4.1 and MS-15-34a are designated as unit 1, but both contain single crystal anomalies that correlated with unit 2 . The average end member concentrations for samples MS-11-4.1 and MS-15-34a were calculated from 5 and 6 crystals, respectively. Figures $31 \mathrm{a}$ and $31 \mathrm{~b}$ show the anorthite and orthoclase concentrations for the crystals within the sample used to calculate the sample averages (solid shapes), the average for each sample (x's) and the single crystal anomalies (open shapes). Error bars are associated with sample standard deviations and single crystal error bars represent standard deviation where there with two analysis points per crystal. 


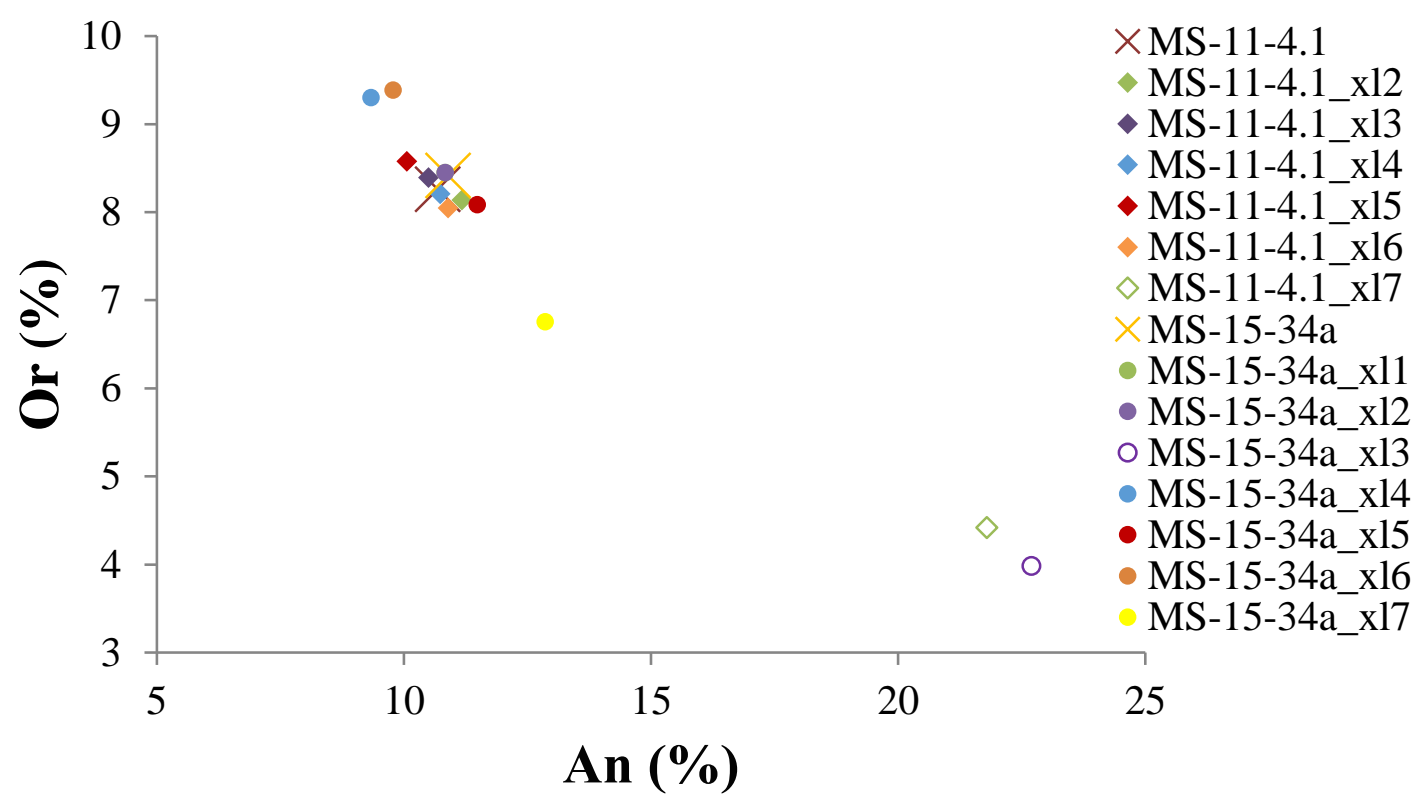

Figure 31a. Anorthite vs orthoclase diagram with single crystal anomalies that correlate to unit 2 when most of the crystals analyzed correlate to unit 1 for samples MS-11-4.1 and MS-15-34a. Solid shapesindividual crystals within a sample. Open shapes - anomalous crystals. X's average sample end member concentrations.

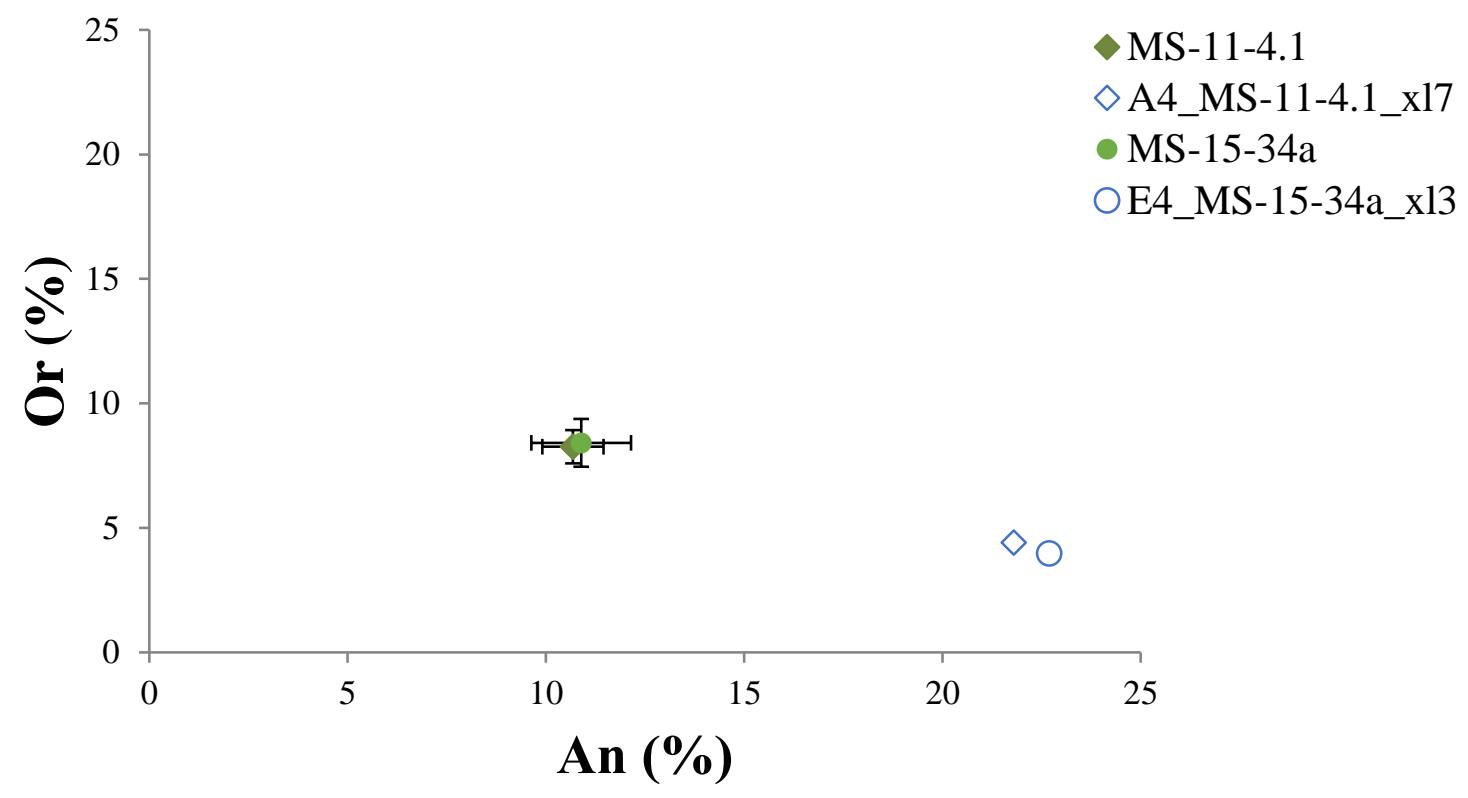

Figure 31b. Anorthite vs orthoclase diagram with single crystal anomalies that correlate to unit 2 when most of the crystals analyzed correlate to unit 1 for samples MS-11-4.1 and MS-15-34a. Error bars are associated with sample standard deviations and single crystal error bars represent standard deviation where there with two analysis points per crystal. 


\section{Non-unit designating feldspars}

Sanidine crystals were found in all units, but only occasionally in units 1-4 $(<2$ crystals) and consistently (>2 crystals) in unit 4 . Anorthoclase crystals, a slightly less potassium enriched feldspar than sanidine but richer in potassium than unit 3 and 4 anorthoclase compositions, were more common in unit 3 with 3 crystals and less common with only single crystals occurring in units 2 and 4 . Albite was rare, only a single crystal occurrence in unit 3 . While all units contain more anorthite richer plagioclase feldspars, they occurred more frequently in unit 2 ( $>3$ crystals) than any other unit ( $<3$ crystals). The occurrence of oligoclase, andesine, labradorite and bytownite within the units suggest the possibility that these feldspars crystallized from more mafic magmas and thus record the input of hotter and more mafic magmas into the rhyolitic reservoirs. This is corroborated by finding pumices and mafic globules ranging from dacite to andesite in unit 2, 3 and 4 (Streck, pers. comm., 2017). 


\section{CONCLUSIONS}

Feldspar compositions successfully correlated samples to cooling units 1,2 and 3/4. Results were consistent with Streck et al., 2015 and tuff units can be traced this way, but lithological and bulk compositions are needed to distinguishing unit 3 and 4 .

Originally determined to have an areal extent of $2,000 \mathrm{~km}^{2}$ by Haddock (1967), this study shows that all outcrops now determined to be Dinner Creek Tuff enclose an area of $\sim 31,300 \mathrm{~km}^{2}$ not including any fallout deposits that likely extended beyond the defined area. Minimal individual unit areal extents decrease with age and are as follows: $\sim 22,590 \mathrm{~km}^{2}$ (unit 1), $\sim 17,920 \mathrm{~km}^{2}$ (unit 2), $\sim 14,170 \mathrm{~km}^{2}$ (unit 3) and $\sim 8,370 \mathrm{~km}^{2}$ (unit 4). Minimal calculated erupted tuff volumes are: $\sim 170 \mathrm{~km}^{3}$ (unit 1), $\sim 125 \mathrm{~km}^{3}$ (unit 2), $\sim 99 \mathrm{~km}^{3}$ (unit 3) and $\sim 46 \mathrm{~km}^{3}$ (unit 4), totaling $\sim 422 \mathrm{~km}^{3}$. Minimal calculated DRE are: $\sim 152 \mathrm{~km}^{3}$ (unit 1), $96 \mathrm{~km}^{3}$ (unit 2), $\sim 78 \mathrm{~km}^{3}$ (unit 3) and $\sim 31$ $\mathrm{km}^{3}$ (unit 4), totaling $\sim 356 \mathrm{~km}^{3}$.

These extents and volumes are the absolute minimal using only locations of exposed tuff sections and the source location. Centering eruptive units on source areas where they are known, extending tuff extents to make a more radial pattern as would be expected for low-aspect ratio, high energy ash-flow tuff eruptions and extending extent beyond several meter-thick outcrops increases the tuff and erupted magma volume. Finally, thick ponded tuff in caldera areas as observed needed to be added. Including probable extents and intra-caldera tuff yields erupted magma volumes of $\sim 375.3$ (unit 1) and $\sim 252.7$ (unit 2). 


\section{REFERENCES}

Anders, M.H. and Sleep, N.H., 1992, Magmatism and extension: the thermal and mechanical effects of the Yellowstone hotspot: Journal of Geophysical Research, v. 97, i. B11, p. 15379-15393.

Anderson, B.S., 2013, Magmatism, metamorphism, and deformation in the Mountain Home Metamorphic Complex, Blue Mountains Province, Oregon, and its role in late Jurassic deformation in the in the western North American Cordillera [MS thesis]: Tuscaloosa, University of Alabama, $181 \mathrm{pp}$.

Armstrong, R.L., Leeman, W.P. and Malde, H.E., 1975, K-Ar dating, Quaternary and Neogene volcanic rocks of the Snake River Plain Idaho: American Journal of Science, v. 275 , p. $225-251$.

Armstrong, R.L., Taubeneck, W.H. and Hales, P.O., 1977, Rb-Sr and K-Ar geochronometry of Mesozoic granitic rocks and their Sr isotopic composition, Oregon, Washington, and Idaho: Geological Society of America Bulletin, v. 88, p. 397-411.

Ashley, R.P., 1995, Petrology and deformation history of the Burnt River Schist and associated plutonic rocks in the Burnt River Canyon area, northeastern Oregon, in Vallier, T.L. and Brooks, H.C. (Eds.), Geology of the Blue Mountains Region of Oregon, Idaho, and Washington: Petrology and Tectonic Evolution of Pre-Tertiary Rocks of the Blue Mountains Region: U.S. Geological Survey Professional Paper 1438 , p. 457-495.

Ave Lallemant, H.G., 1995, Pre-Cretaceous tectonic evolution of the Blue Mountains Province, northeastern Oregon, in Vallier, T.L. and Brooks, H.C. (Eds.), Geology of the Blue Mountains region of Oregon, Idaho, and Washington: petrology and tectonic evolution of pre-Tertiary rocks of the Blue Mountains region: U.S. Geological Survey Professional Paper 1438, p. 271-304.

Ave Lallemant, H.G., Schmidt, W.J. and Kraft, J. L., 1985, Major Late-Triassic strikeslip displacement in the Seven Devils Terrane, Oregon and Idaho: a result of leftoblique plate convergence: Tectonophysics, v. 119, p. 299-328.

Barry, T.L., Kelley, S.P., Reidel, S.P., Camp, V.E., Self, S., Jarboe, N.A., Duncan, R.A. and Renne, P.R., 2013, Eruption chronology of the Columbia River Basalt Group, in Reidel, S.P., Camp, V.E., Ross, M.E., Wolff, J.A., Martin, B.S., Tolan, T.L. and Wells, R.E. (Eds.), The Columbia River Flood Basalt Province: Geological Society of America Special Paper 497, p. 45-66, doi:10.1130/2013.2497(02). 
Bailey, D.G., 1990, Geochemistry and petrogenesis of Miocene volcanic rocks in the Powder River volcanic field, northeastern Oregon [Ph.D. Thesis]: Pullman, Washington State University, 341 pp.

Bishop, E.M., 1995a, High-pressure, low-temperature schistose rocks of the Baker Terrane, northeastern Oregon, in Vallier, T.L., and Brooks, H.C. (Eds.), Geology of the Blue Mountains region of Oregon, Idaho, and Washington: petrology and tectonic evolution of pre-Tertiary rocks of the Blue Mountains region: U.S. Geological Survey Professional Paper 1438, p. 211-220.

Bishop, E.M., 1995b, Mafic and ultramafic rocks of the Baker Terrane, eastern Oregon, and their implications for terrane origin, in Vallier, T.L., and Brooks, H.C. (Eds.), Geology of the Blue Mountains region of Oregon, Idaho, and Washington: petrology and tectonic evolution of pre-Tertiary rocks of the Blue Mountains region: U.S. Geological Survey Professional Paper 1438, p. 221-246.

Benson, T.R. and Mahood, G.A., 2016, Geology of the Mid-Miocene Rooster Comb Caldera and Lake Owyhee Volcanic Field, eastern Oregon: Silicic volcanism associated with Grande Ronde flood basalt: Journal of Volcanology and Geothermal Research, v. 309, p. 96-117.

Benson, T.R., Mahood, G.A. and Grove, M., 2017, Geology and ${ }^{40} \mathrm{Ar} /{ }^{39} \mathrm{Ar}$ geochronology of the middle Miocene McDermitt volcanic field, Oregon and Nevada: Silicic volcanism associated with propagating flood basalt dikes at initiation of the Yellowstone hotspot: Geological Society of America Bulletin, v. 129; no. 9/10; p. 1027-1051, https://doi .org /10 .1130/B31642.1.

Bowen, N.L., 1956, The geology of the Beulah area, Malheur County Oregon [MS thesis]: Eugene, University of Oregon, $80 \mathrm{pp}$.

Brooks, H.C., McIntyre, J.R. and Walker, G.W., 1976, Geologic Map of the Oregon part of the Baker $1^{\circ} \times 2^{\circ}$ Quadrangle: Oregon Department of Geology and Mineral Industries Geologic Map Series GMS-7, scale 1:250,000.

Brooks, H.C. and Vallier, T.L., 1978, Mesozoic rocks and tectonic evolution of eastern Oregon and western Idaho, in Howell, D.G., and Mc-Dougall, K.A. (Eds.), Mesozoic paleogeography of the western United States: Society of Economic Paleontologists and Mineralogists, Pacific Section, Paleogeography Symposium 2, p. 133-145.

Brueseke, M.E., Hart, W.K. and Heizler, M.T., 2008, Diverse mid-Miocene silicic volcanism associated with the Yellowstone-Newberry thermal anomaly: Bulletin of Volcanology, v. 70, p. 343-360. 
Bryan, S.E. and Ernst, R.E., 2008, Revised definition of Large Igneous Provinces (LIPs): Earth Science Reviews, v. 86, p. 175-202.

Camp, V.E., 1995, Mid-Miocene propagation of the Yellowstone mantle plume head beneath the Columbia River basalt source region: Geology, v. 23, n. 5, p. 435-438.

Camp, V.E. and Ross, M.E., 2004, Mantle dynamics and genesis of mafic magmatism in the intermontane Pacific Northwest: Journal of Geophysical Research, v. 109, B08204, p. 1-14, doi:10.1029/2003JB002838.

Camp, E.V., Ross, M.R. and Hanson, W. E., 2002, Genesis of flood basalts and Basin and Range volcanic rocks from Steens Mountain to the Malheur River Gorge, Oregon: Geological Society of America Bulletin, v. 114, n. 12, p. 105-128, doi:10.1130/00167606.

Christiansen, R. L., 1984, Yellowstone magmatic evolution: Its bearing on understanding large-volume explosive volcanism, in National Research Council Studies in Geophysics (Eds.), Explosive Volcanism: Inception, Evolution, and Hazards. National Academy of Sciences, Washington, D. C., p. 84-95.

Christiansen, R.L., 2001, The Quaternary and Pliocene Yellowstone Plateau volcanic field of Wyoming, Idaho, and Montana: US Geological Survey Professional Paper $729-G, 120 \mathrm{pp}$.

Christiansen, R.L. and Yeats, R.S., 1992, Post-Laramide geology of the U.S. Cordilleran region, in Burchfiel, B.C., et al. (Eds.), The Cordilleran orogeny: Conterminous U.S.: Geological Society of America, Geology of North America, v. G3, p. 261-406.

Christiansen, R.L., Foulger, C.R. and Evans, J.R., 2002, Upper-mantle origin of the Yellowstone hotspot: Geological Society of America Bulletin, v. 114, p. 1245-1256.

Coble, M.A. and Mahood, G.A., 2012, Initial impingement of the Yellowstone plume located by widespread silicic volcanism contemporaneous with Columbia River flood basalts: Geology, v. 40, no. 7, p. 655-658.

Coble, M.A. and Mahood, G.A., 2016, Geology of the High Rock caldera complex, northwest Nevada, and implications for intense rhyolitic volcanism associated with flood basalt magmatism and the initiation of the Snake River Plain-Yellowstone trend: Geosphere, v. 12, n. 1, p. 58-113, doi: 10.1130/GES01162.1.

Coffin, M.F. and Eldholm, O., 1994, Large igneous provinces: crustal structure, dimensions, and external consequences: Reviews of Geophysics, v. 32, i. 1, p. 1-36. doi:10.1029/93RG02508. 
Coffin, M.F. and Eldholm, O., 2001, Large igneous provinces: progenitors of some ophiolites?, in Mantle plumes: their classification through time, R.E. Ernst K.L. and Buchan (Eds.): Geological Society of America, Special Paper 352, p. 59-70.

Coffin, M.F. and Eldholm, O., 2005. Large igneous provinces, in: Selley, R.C., Cocks, R., Plimer, I.R. (Eds.), Encyclopedia of Geology, Elsevier, Oxford, p. 315-323.

Courtillot, V.E. and Renne, P.R., 2003, On the ages of flood basalt events: Comptes Rendus Geoscience, v. 335, i. 1, p. 113-140, doi:10.1016/S1631-0713(03)00006-3.

Criss, R.E. and Fleck, R.J., 1987, Petrogenesis, geochronology, and hydrothermal systems of the northern Idaho batholith and adjacent areas based on ${ }^{18} \mathrm{O} /{ }^{16} \mathrm{O}, \mathrm{D} / \mathrm{H}$, ${ }^{87} \mathrm{Sr} /{ }^{86} \mathrm{Sr}$, and ${ }^{40} \mathrm{Ar} /{ }^{39} \mathrm{Ar}$ studies: U.S. Geological Survey Professional Paper 1436, $\mathrm{p}$. 95-138.

Cruz, M., 2017, Field Mapping Investigation and Geochemical Analysis of Volcanic Units within the Dinner Creek Tuff Eruptive Center, Malheur County, Eastern Oregon [MS thesis], Portland, Portland State University, 217 pp.

Cummings, M.L., Evans, J.G. and Ferns, M.L., 1994, Stratigraphic and structural evolution of the middle Miocene Oregon-Idaho graben Malheur County, Oregon, in Swanson, D.A. and Haugerud, R.S. (Eds.), Geologic field trips in the Pacific Northwest, 1994 Geological Society of America Annual Meeting, Seattle, Washington, v. 1, p. 1G-1-1G-20.

Cummings, M.L., Evans, J.G., Ferns, M.L. and Lees, K.R., 2000, Stratigraphic and structural evolution of the middle Miocene synvolcanic Oregon-Idaho graben: Geological Society of America Bulletin, v. 112, n. 5, p. 668-682.

Cummings, M.L., Johnson, A.G. and Cruikshank, K.M., 1996, Intragraben fault zones and hot-spring deposits in the Oregon-Idaho graben: a geophysical study, in Coyner, A.R., and Fahey, P.L. (Eds.), Geology and Ore Deposits of the American Cordillera: Geological Society of Nevada Symposium Proceedings, Reno/Sparks, Nevada, April 1995, p. 1047-1062.

Davenport, R.E., 1971, Geology of the Rattlesnake and Older Ignimbrites in the Paulina Basin and Adjacent Area, Central Oregon [Ph.D. thesis]: Corvallis, University of Oregon, $132 \mathrm{pp}$.

Dickinson, W.R., 1979, Mesozoic forearc basin in central Oregon: Geology, v. 7, n. 4, p. $166-170$. 
Dole, H.M. and Corcoran, R.E., 1954, Reconnaissance geology along U.S. Highway 20 between Vale and Duchanan, Malheur and Harney Counties, Oregon: Oregon Department of Geology and Mineral Industries, Ore-Bin, v. 16, n. 6, p. 37-39.

Dorsey, R.J. and LaMaskin, T.A., 2007, Stratigraphic Record of Triassic-Jurassic Collisional Tectonics in the Blue Mountains Province, Northeastern Oregon: American Journal of Science, v. 307, p. 1167-1193.

Dorsey, R.J. and LaMaskin, T.A., 2008, Mesozoic collision and accretion of oceanic terranes in the Blue Mountains province of northeastern Oregon: New insights from the stratigraphic record, in Spencer, J.E. and Titley, S.R. (Eds.), Ores and orogenesis: Circum-Pacific tectonics, geologic evolution, and ore deposits: Arizona Geological Society Digest, v. 22, p. 325-332.

Draper, D.S., 1991, Late Cenozoic bimodal magmatism in the northern Basin and Range Province of Southeastern Oregon: Journal of Volcanology and Geothermal Research, v. 47, p. 299-328.

Edwards, J.H., 2013, Structural controls of the Neal Hot Springs geothermal system, eastern Oregon [MS thesis]: Reno, University of Nevada-Reno, 83 pp.

Elison, M.W., Speed, R.C. and Kistler, R.W., 1990, Geologic and isotopic constraints on the crustal structure of the northern Great Geologic and isotopic constraints on the crustal structure of the northern Great Basin: Geological Society of America Bulletin, v. 8, p. 1077-1092, doi.org/10.1130/0016-7606(1990)102b1077.

Ernst, R.E. and Buchan, K.L. 2001, Large mafic magmatic events through time and links to mantle-plume heads, in Mantle plumes: their identification through time, R.E. Ernst and K.L. Buchan (Eds.): Geological Society of America, Special Paper 352, p. 483-575.

Ernst, R.E., Buchan, K.L. and Campbell, I.H., 2005, Frontiers in Large Igneous Province research: Lithos, i. 79, p. 271-297, doi: 10.1016/j.lithos.2004.09.004.

Evans, J.G., 1992, Geologic Map of the Dooley Mountain 7 1⁄2' Quadrangle, Baker County, Oregon: U.S. Geological Survey Geologic Quadrangle Map, GQ-1694, 9 p., scale 1:24,000.

Evans, J.G. and Binger, G.B., 1999, Geologic map of the Little Black Canyon quadrangle, Malheur County, Oregon: U.S. Geological Survey Open-File Report 98493, 12-p. text, 1 sheet, scale 1:24,000. [Also available at https://pubs.er.usgs.gov/publication/ofr98493.] 
Evans, J.G., Griscom, A., Halvorson, P.F. and Cummings, M.L., 2002, Tracking the Western Margin of the North American Craton beneath Southeastern Oregon: a multidisciplinary approach: Idaho Geological Survey Bulletin, v. 30, p. 35-57.

Farmer, G.L. and DePaolo, D.J., 1983, Origin of Mesozoic and Tertiary granite in the western United States and implications for Pre-Mesozoic crustal structure: 1. Nd and Sr isotopic studies in the geocline of the Northern Great Basin: Journal of Geophysical Research, v. 88 i. B4, p. 3379-3401, doi.org/10.1029/JB088iB04p03379.

Ferns, M.L. and Brooks, H.C., 1995, The Bourne and Greenhorn subterranes of the Baker Terrane, northeastern Oregon: Implications for the evolution of the Blue Mountains island-arc system, in Vallier, T.L. and Brooks, H.C. (Eds.), Geology of the Blue Mountains Region of Oregon, Idaho and Washington: Petrology and Tectonic Evolution of Pre-Tertiary Rocks of the Blue Mountains Region: U.S. Geological Survey Professional Paper 1438, p. 331-358.

Ferns, M.L. and McClaughry, J.D., 2013, Stratigraphy and volcanic evolution of the middle Miocene to Pliocene La Grande-Owyhee eruptive axis in eastern Oregon: Geological Society of America Memoir 497, p. 401-427, doi.org/10.1130/2013.2497(16).

Ferns, M.L., Streck, M.J. and McClaughry, J.D., 2017, Field-Trip Guide to Columbia River Flood Basalts, Associated Rhyolites, and Diverse Post-Plume Volcanism in Eastern Oregon: USGS Scientific Investigations Report 2017-5022-O, 71 pp.

Fleck, R.J. and Criss, R.E., 1985, Strontium and oxygen isotopic variations in Mesozoic and Tertiary plutons of central Idaho: Contributions to Mineralogy and Petrology, v. 90, p. 291-308.

Fleck, R.J. and Criss, R.E., 2007, Location, age, and tectonic significance of the Western Idaho Suture Zone: U.S. Geological Survey Professional Paper 1738, 202 pp.

Follo, M.F., 1986, Sedimentology of the Wallowa Terrane, Northeastern Oregon [Ph.D. Dissertation]: Cambridge, Harvard University, 292 pp.

Follo, M.F., 1992, Conglomerates as clues to the sedimentary and tectonic evolution of a suspect terrane: Wallowa Mountains, Oregon: Geological Society of America Bulletin, v. 104, p. 1561-1576.

Follo, M.F., 1994, Sedimentology and stratigraphy of the Martin Bridge Limestone and Hurwal Formation (Upper Triassic to Lower Jurassic) from the Wallowa Terrane, Oregon, in Vallier, T.L. and Brooks, H.C. (Eds.), Stratigraphy, Physiography, and Mineral Resources of the Blue Mountains Region: U.S. Geological Survey

Professional Paper 1439, p. 1-27. 
Ford, M.T., Grunder, A.L. and Duncan, R.A., 2013, Bimodal volcanism of the High Lava Plains and Northwestern Basin and Range of Oregon: Extent and tectonic implications of age-progressive rhyolites: Geochemisty, Geophysics, Geosystems, v. 14 , n. 8 , p. $2836-2853$.

Freundt, A. and Rosi, M., 1998, From magma to tephra: modelling physical processes of explosive volcanic eruptions: Amsterdam, Elsevier Science B.V, 318 pp.

Gaschnig, R.M., Vervoort, J.D., Lewis, R.S. and Tikoff, B., 2011, Isotopic evolution of the Idaho Batholith and Challis Intrusive Province, Northern US Cordillera: Journal of Petrology, v. 52, i. 12, p. 2397-2429, doi.org/10.1093/petrology/egr050.

Getty, S.R., Selverstone, J., Wernicke, B.P., Jacobsen, S.B., Aliberti, E. and Lux, D.R., 1993, Sm-Nd dating of multiple garnet growth events in an arc-continent collision zone, northwestern U.S. Cordillera: Contributions to Mineralogy and Petrology, n. 115, p. 45-57.

Gilully, J., 1937, Geology and Mineral Resources of the Baker Quadrangle, Oregon: US Geological Survey Bulletin 879, p. 1-119.

Glen, J.M.G. and Ponce, D.A., 2002, Large-scale fractures related to inception of the Yellowstone hotspot: Geology, v. 30, p. 647-650.

Gray, K.D. and Oldow, J.S., 2005, Contrasting structural histories of the Salmon River belt and Wallowa terrane: Implications for terrane accretion in northeastern Oregon and west-central Idaho: Geological Society of America Bulletin, v. 117, p. 687-706.

Gray, W.L., 1956, Geology of Drinking Water Pass area, Harney and Malheur Counties, Oregon [MS Thesis]: Eugene, University of Oregon, 165 pp.

Haddock, G.H., 1967, The Dinner Creek Welded Ash-Flow Tuff of the Malheur Gorge Area, Malheur County, Oregon [Ph.D. thesis]: Eugene, University of Oregon, 111 pp.

Hess, E.N., 2014, Strontium, Lead, and Oxygen Isotopic Signatures of Mid-Miocene Silicic Volcanism in Eastern Oregon [M.S. thesis]: Portland, Portland State University, $102 \mathrm{pp}$.

Hooper, P.R., Binger, G.B. and Lees, K.R., 2002, Ages of the Steens and Columbia River flood basalts and their relationship to extension-related calc-alkalic volcanism in eastern Oregon: Geological Society of America Bulletin, v. 114, n. 1, p. 43-50.

Hooper, P.R., Camp, V.E., Reidel, S.P. and Ross, M.E., 2007, The origin of the Columbia River flood basalt province: Plume versus nonplume models, in Foulger, G.R., and Jurdy, D.M. (Eds.), Plates, plumes, and planetary processes: Geological Society of America Special Paper 430, p. 635-668. 
Hooper, P.L. and Swanson, D. A., 1990, The Columbia River Basalt Group and associated volcanic rocks of the Blue Mountains Province, in G. W. Walker (Ed.), Geology of the Blue Mountains region of Oregon, Idaho, and Washington: Cenozoic geology of the Blue Mountains Region: U.S. Geological Survey Professional Paper 1437, p. 63-99.

Humphreys, E.D. and Coblentz, D.D., 2007, North American dynamics and western U.S. tectonics: Reviews of Geophysics, v. 45, i. 3, p. 1-30, doi:10.1029/2005RG000181.

Jarboe, N.A., Coe, R.S., Renne, P.R. and Glen, J.M.G., 2010, The age of the Steens reversal and the Columbia River Basalt Group: Chemical Geology, v. 274, p. 158168, doi: 10.1016/j.chemgeo.2010.04.001.

John, D.A., Wallace, A.R., Ponce, D.A., Fleck, R.B. and Conrad, J.E., 2000, New perspectives on the geology and origin of the northern Nevada rift, in Cluer, J.K., Price, J.G., Struhsacker, E.M., Hardyman, R.F. and Morris, D.L. (Eds.), Geology and Ore deposits 2000: The Great Basin and Beyond: Geological Society of Nevada Symposium Proceedings, May 15-18, pp. 127-154.

Jordan, B.J., Grunder, A.L., Duncan, R. and Deino, A., 2004, Geochronology of age progressive volcanism of the Oregon High Lava Plains: implications for the plume interpretation of Yellowstone: Journal of Geophysical Research, v. 109, i. B10, 19 pp., B10202, doi:10.1029/2003JB002726.

Kistler, R.W. and Lee, D.E., 1989, Rubidium, strontium, and strontium isotopic data for a suite of granitoid rocks from the Basin and Range Province, Arizona, California, Nevada, and Utah: U.S. Geological Survey Open-File Report 89-199, pp. 13.

Kittleman, L.R., Green, A.R., Haddock, G.H., Hagood, A.R., Johnson, A.M., McMurray, J.M., Russell, R.G. and Weeden, D.A., 1965, Cenozoic stratigraphy of the Owyhee region, south-eastern Oregon: University of Oregon Museum of Natural History Bulletin, n. 1, 45 pp.

Kittleman, L.R., Green, A.R., Haddock, G.H, Hagood, A.R., Johnson, A.M., McMurray, J.M., Russell, R.G. and Weeden, D.A., 1967, Geologic map of the Owyhee region, Malheur County, Oregon: University of Oregon Museum of Natural History Bulletin, n. 8, scale 1: 125,000.

Kuntz, M.A., Covington, H.R. and Schorr, L.J., 1992, An overview of basaltic volcanism of the eastern Snake River Plain, in Link, P.K., Kuntz, M.A. and Platt, L.B. (Eds.), Regional Geology of Eastern Idaho and Western Wyoming: Geological Society of America Memoir, v. 179, p. 227-267. 
Kurz, G.A., Schmitz, M.D., Northrup, C.J. and Vallier, T.L., 2012, U-Pb geochronology and geochemistry of intrusive rocks from the Cougar Creek Complex, Wallowa arc terrane, Blue Mountains Province, Oregon-Idaho: Geological Society of America Bulletin, v. 124, n. 3-4, p. 578-595.

LaMaskin, T.A., 2009, Stratigraphy, Provenance, and Tectonic Evolution of Mesozoic Basins in the Blue Mountains Province, Eastern Oregon and Western Idaho [Ph.D. thesis]: Eugene, University of Oregon, 232 pp.

Large, A.M., 2016, Columbia River Basalt Rhyolite Flare-up: Rhyolites of Buchanan Volcanic Complex and Dooley Mountain Volcanic Complex, Oregon [M.S. thesis]: Portland, Portland State University, 175 pp.

Leeman, W.P., Annen, C. and Dufek, J., 2008, Snake River Plain-Yellowstone silicic volcanism: implications for magma genesis and magma fluxes, in Annen, C. and Zellmer, G.F (Eds.), Dynamics of Crustal Magma Transfer, Storage and Differentiation: Geological Society of London Special Publication, v. 304, p. 235 259, doi.org/10.1144/SP304.12.

Lees, K.R., 1994, Magmatic and tectonic changes through time in the Neogene volcanic rocks of the Vale area, Oregon, northwestern U.S.A. [Ph.D. dissertation]: Milton Keynes, United Kingdom, The Open University, 282 pp.

Lipman, P.W., Prostka, H.J. and Christiansen, R.L., 1972, Cenozoic volcanism and plate-tectonic evolution of the western United States. I. early and middle Cenozoic: Philosophical Transactions of the Royal Society of London. Series A, Mathematical and Physical Sciences, v. 271, n. 1213, p. 217-248.

Lund, K., and Snee, L. W., 1988, Metamorphism, structural development, and age of the continent-island arc juncture in west-central Idaho, in Ernst, W. G. (Ed.), Metamorphism and crustal evolution of the western United States, Rubey Volume III: Englewood Cliffs, New Jersey, Prentice Hall, p. 297-331.

MacLeod, N.S., Walker, G.W. and McKee, E.H., 1976, Geothermal significance of eastward increase in age of upper Cenozoic rhyolitic domes in southeastern Oregon: Proceedings of the Second United Nations Symposium on the Development and Use of Geothermal Resources 1, U.S. Government Printing Office, Washington, D.C, p. 456-474.

Mahood, G.A. and Benson, T.R, 2017, Using ${ }^{40} \mathrm{Ar} /{ }^{39} \mathrm{Ar}$ ages of intercalated silicic tuffs to date flood basalts: Precise ages for Steens Basalt Member of the Columbia River Basalt Group: Earth and Planetary Science Letters, v. 459, p. 340-351, doi.org/10.1016/j.eps1.2016.11.038. 
Manduca, C.A., Silver, L.T. and Taylor, H.P., $1992,{ }^{87} \mathrm{Sr} /{ }^{86} \mathrm{Sr}$ and ${ }^{18} \mathrm{O} /{ }^{16} \mathrm{O}$ isotopic systematics and geochemistry of granitoid plutons across a steeply-dipping boundary between contrasting lithospheric blocks in western Idaho: Contributions to Mineralogy and Petrology, v. 109, p. 355-372.

Marcy, P.I., Streck, M.J. and Ferns, M.L., 2013, Mid-Miocene silicic volcanism of the Three Fingers-Mahogany Mountain area, SE Oregon-Revisited [abs.]: American Geophysical Union Abstract, n. V23C-2487.

Nash, B.P. and Perkins, M.E., 2012, Neogene fallout tuffs from the Yellowstone Hotspot in the Columbia Plateau region, Oregon, Washington, and Idaho, USA: PLoS ONE 7(10): e44205. doi: 10.1371/journal.pone.0044205.

Nash, B.P., Perkins, M.E., Christensen, M.E., Lee, D.C. and Halliday, A.N., 2006, The Yellowstone hotspot in space and time: $\mathrm{Nd}$ and $\mathrm{Hf}$ isotopes in silicic magmas: Earth and Planetary Science Letters, v. 247, p. 143-156.

Oldow, J.S., Bally, A.W., Lallemant, H.G.A. and Leeman, W.P., 1989, Phanerozoic evolution of the North American Cordillera: United States and Canada, in Bally, A.W. and Palmer, A.R. (Eds.), The geology of North America: an overview: Geological Society of America, The Geology of North America, v. A, p. 139-232.

Pardee, J.T. and Hewett, D.F., 1941, Preliminary geologic map of the Sumpter quadrangle, in Mineral Resources of Oregon: Oregon Bureau of Mines and Geology, v. 1, n. 6,128 p.

Parsons, T., Thompson, G.A. and Sleep, N.H., 1994, Mantle plume influence on the Neogene uplift and extension of the U.S. western Cordillera?: Geology, v. 22, p. 8386.

Perkins, M.E and Nash, B.P., 2002, Explosive silicic volcanism of the Yellowstone hotspot: the ash fall tuff record: Geological Society of America Bulletin, v.114, n. 3, p. $367-381$.

Pierce, K.L. and Morgan, L.A., 1990, The track of the Yellowstone hot spot: volcanism, faulting, and uplift: U.S. Geological Survey Open-File Report 90-415, 49 pp.

Pierce, K.L. and Morgan, L.A., 1992, The track of the Yellowstone hot spot: Volcanism, faulting, and uplift, in Link, P.K., et al. (Eds.), Regional geology of eastern Idaho and western Wyoming: Geological Society of America Memoir, v. 179, p. $1-53$.

Pierce, K.L. and Morgan, L.A., 2009, Is the track of the Yellowstone hotspot driven by a deep mantle plume? Review of volcanism, faulting, and uplift in light of new 
data: Journal of Volcanology and Geothermal Research, v. 188, p. 1-25, doi: 10.1016/j.jvolgeores.2009.07.009.

Pierce, K.L., Morgan, L.A. and Saltus, R.W., 2002, Yellowstone plume head: postulated tectonic relations to the Vancouver slab, continental boundaries, and climate, in Bonnichsen, B., White, C.M. and McCurry, M. (Eds.), Tectonic and Magmatic Evolution of the Snake River Plain Volcanic Province: Idaho Geological Survey Bulletin, v. 30, p. 5-33.

Reef, J.W., 1983, The Unity Reservoir rhyodacite tuff-breccia and associated volcanic rocks, Baker County, Oregon [Ph.D. Thesis]: Pullman, Washington State University, $128 \mathrm{pp}$.

Reidel, S.P., 1998, Emplacement of Columbia River flood basalt: Journal of Geophysical Research, v. 103, p. 27,393-27,410, doi:10.1029/97JB03671.

Reidel, S.P., 2005, A lava flow without a source: The Cohasset flow and its compositional components, Sentinel Bluffs Member, Columbia River Basalt Group: The Journal of Geology, v. 113, p. 1-21, doi:10.1086/425966.

Reidel, S.P., Camp, V.E., Tolan, T.L. and Martin, B.S., 2013, The Columbia River flood basalt province: stratigraphy, areal extent, volume, and physical volcanology, in Reidel, S.P., Camp, V.E., Ross, M.E., Wolff, J.A., Martin, B.S., Tolan, T.L. and Wells, R.E. (Eds.), The Columbia River Flood Basalt Province: Geological Society of America Special Paper 497, p. 1-43.

Reidel, S.P. and Tolan, T.L., 2013, The late Cenozoic evolution of the Columbia River system in the Columbia River flood basalt province, in Reidel, S.P., Camp, V.E., Ross, M.E., Wolff, J.A., Martin, B.S., Tolan, T.L. and Wells, R.E. (Eds.), The Columbia River Flood Basalt Province: Geological Society of America Special Paper 497, p. 201-230.

Reidel, S.P., Tolan, T.L., Hooper, P.R., Beeson, M.H., Fecht, K.R., Bentley, R.D. and Anderson, J.L., 1989, The Grande Ronde Basalt, Columbia River Basalt Group: Stratigraphic descriptions and correlations in Washington, Oregon, and Idaho, in Reidel, S.P. and Hooper, P.R. (Eds.), Volcanism and Tectonism in the Columbia River Flood-Basalt Province: Geological Society of America Special Paper 239, p. 21-53.

Rowley, P.W., 2001, The Cenozoic evolution of the Great Basin area, U.S.A.- new interpretations based on regional geologic mapping, in Erskine, M.C. (Ed.), The Geologic Transition, High Plateaus to Great Basin-A Symposium and Field Guide (The Mackin Volume): Utah Geological Association Publication, v. 30, p. 169-188. Rytuba, J.J. and McKee, E.H., 1984, Peralkaline ash flow tuffs and calderas of the McDermitt Volcanic Field, southeast Oregon and north central Nevada: Journal of Geophysical Research, v. 89, i. B10, p. 8616-8628. 
Rytuba, J.J. and Vander Meulen, D.B., 1991, Hot-spring precious-metal systems in the Lake Owyhee volcanic field, Oregon-Idaho, in Raines, G.L., Lisle, R.E., Schafer, R.W. and Wilkinson, W.H. (Eds.), 'Geology and ore deposits of the Great Basin', Reno, Geological Society of Nevada, Symposium Proceedings, April 1 - 5, 1990, v. 2, p. $1085-1096$.

Rytuba, J.J., Vander Meulen, D.B., Plouff, D. and Minor, S.A., 1985, Geology of the Mahogany Mountain caldera, Oregon [abs.]: Geological Society of America Abstracts with Programs, v. 17, n. 4, p. 263.

Schwartz, J.J., Johnson, K., Miranda, E.A. and Wooden, J.L., 2011b, The generation of high $\mathrm{Sr} / Y$ plutons following Late Jurassic arc-arc collision, Blue Mountains province, NE Oregon: Lithos, v. 126, p. 22-41.

Schwartz, J.J., Snoke, A.W., Cordey, F., Johnson, K., Frost, C.D., Barnes, C.G., LaMaskin, T.A and Wooden, J.L., 2011a, Late Jurassic magmatism, metamorphism, and deformation in the Blue Mountains Province, northeast Oregon: Geological Society of America Bulletin, v. 123, n. 9/10, p. 2083-2111, doi: 10.1130/B30327.1.

Schwartz, J.J., Snoke, A.W., Frost, C.D., Barnes, C.G., Gromet, L.P. and Johnson, K., 2010, Analysis of the Wallowa-Baker terrane boundary: Implications for tectonic accretion in the Blue Mountains province, northeastern Oregon: Geological Society of America Bulletin, v. 122, n. 3/4, p. 517-536.

Selverstone, J., Wernicke, B.P. and Aliberti, E.A., 1992, Intracontinental subduction and hinged unroofing along the Salmon River Suture Zone, West Central Idaho: Tectonics, v. 11, n. 1, p. 124-144.

Shervais, J.W. and Hanan, B.B., 2008, Lithospheric topography, tilted plumes, and the track of the Snake River-Yellowstone hot spot: Tectonics, v. 27, i. 5, doi:10.1029/2007TC002181.

Shoemaker, K.A. and W.K. Hart, 2002, Temporal controls on basalt genesis and evolution on the Owyhee Plateau, Idaho and Oregon, in Bonnichsen, B., White, C.M. and McCurry, M. (Eds.), Tectonic and Magmatic Evolution of the Snake River Plain Volcanic Province: Idaho Geological Survey Bulletin, v. 30, p. 313-328.

Sigurdsson, H., Houghton B. F., McNutt, S.R., Rymer, H. and Stix, J., eds., 2015, Encyclopedia of Volcanoes 2nd Edition: Boston, Academic Press, 1456 pp.

Smith, R.B. and Arabasz, W.J., 1991, Seismicity of the Intermountain Seismic Belt, in Slemmons, D.B., Engdahl, E.R., Zoback, M.L. and Blackwell, D.D. (Eds.), Neotectonics of North America: Geological Society of America, Boulder, CO, p. $185-228$. 
Smith, R.B. and Braile, L.W., 1984, Crustal structure and evolution of an explosive silicic volcanic system at Yellowstone National Park, Studies in Geophysics: Explosive Volcanism: Inception, Evolution, and Hazards, National Academy Press, Washington D.C, p. 96-109.

Smith, R.B. and Braile, L.W., 1994, The Yellowstone hotspot: Journal of Volcanology and Geothermal Research, v. 61, i. 3-4, p. 121-187.

Smith, R.L. and Luedke, R.G., 1984, Potentially active volcanic lineaments and loci in western conterminous United States, in, Explosive Volcanism: Inception, Evolution and Hazards, National Research Council, p. 47-66, National Academic Press, Washington, D. C.

Smith, R.B. and Sbar, M., 1974, Contemporary tectonics and seismicity of the Western United States with emphasis on the Intermountain Seismic Belt: Geological Society of America Bulletin, v. 85, p. 1205-1218.

Smith. R.B., Jordan, M., Steinberger, B., Puskas, C.M., Farrell, J., Waite, G. P., Husen, S., Chang, W. and O'Connell, R., 2009, Geodynamics of the Yellowstone hotspot and mantle plume: Seismic and GPS imaging, kinematics, and mantle flow: Journal of Volcanology and Geothermal Research, v. 188, p. 26-56.

Smith, R.B., Richins, W.D., Doser, D.I., Smith, R.B., Richins, W.D. and Doser, D.I., 1985, The 1983 Borah Peak, Idaho, earthquake: Regional seismicity, kinematics of faulting, and tectonic mechanism. Workshop XXVIII on the "Borah Peak Earthquake”: U.S. Geological Survey Open-File Report 85-290, p. 236-263.

Snee, L.W., Davidson, G.F. and Unruh, D.M., 2007, Geologic, geochemical and ${ }^{40} \mathrm{Ar} /{ }^{39} \mathrm{Ar}$ and $\mathrm{U}-\mathrm{Pb}$ thermochrolonogic constraints for the tectonic development of the Salmon River suture zone near Orofino, Idaho in Kuntz, M.A. and Snee, L.W. (Eds.), Geological Studies of the Salmon River Suture Zone and Adjoining Areas, WestCentral Idaho and Eastern Oregon: U.S. Geological Survey Professional Paper 1738, p. 51-94.

Streck, M.J., and Ferns, M., 2012, The Rhyolite Flare-up of the Columbia River Basalt Province and its Bearing on Plume vs. Non-plume Models, American Geophysical Union, Fall Meeting 2012, abstract \#DI53A-2372.

Streck, M.J., Ferns, M. and McIntosh H., 2015, Large, persistent rhyolitic magma reservoirs above Columbia River Basalt storage sites: The Dinner Creek Tuff Eruptive Center, eastern Oregon: Geosphere, v. 11 no. 2, p. 1-10. 
Streck, M.J. and Grunder, A.L, 1995, Crystallization and welding variations in a widespread ignimbrite sheet; the Rattlesnake Tuff, eastern Oregon, USA: Bulletin of Volcanology, v. 57, p. 151-169.

Takahahshi, E., Katsuji, N. and Wright, T.L., 1998, Origin of the Columbia River basalts: melting model of a heterogeneous plume head: Earth and Planetary Science Letters, v. 162, i. 1-4, 63-80.

Tolan, T.L., Reidel, S.P., Beeson, M.H., Anderson, J.L., Fecht, K.R. and Swanson, D.A., 1989, Revisions to the estimates of the areal extent and volume of the Columbia River Basalt Group: Geological Society of America Special Paper 239, p. 1-20.

Thompson, G.A., 1998, Deep mantle plumes and geoscience vision: GSA Today, v. 8, n. 4, p. $7-25$.

Tumpane, K., Crowley, J., Schmitz, M. and Northrup, C.J., 2008, New geochronological constraints on the deposition of the Huntington Formation, Olds Ferry Terrane, and implications for the evolution of the Blue Mountains Province: Geological Society of America Abstracts with Programs, v. 40, n. 1, p. 47.

Unruh, B.D.M., Lund, K., Kuntz, M.A. and Snee, L.W., 2008, Uranium-lead zircon ages and $\mathrm{Sr}, \mathrm{Nd}$, and $\mathrm{Pb}$ isotope geochemistry of selected plutonic rocks from Western Idaho: U.S. Geological Survey Open-File Report 2008-1142, 13 pp.

Vallier, T.L., 1977, The Permian and Triassic Seven Devils Group, western Idaho and northeast Oregon: U.S. Geological Survey Bulletin 1437, 58 pp.

Vallier, T.L., 1995, Petrology of pre-Tertiary igneous rocks in the Blue Mountains region of Oregon, Idaho, and Washington: Implications for the geologic evolution of a complex island arc, in Vallier, T.L. and Brooks, H.C. (Eds.), Geology of the Blue Mountains region of Oregon, Idaho, and Washington: petrology and tectonic evolution of pre-Tertiary rocks of the Blue Mountains region: U.S. Geological Survey Professional Paper 1438, p. 125-209.

Vander Meulen, D.B., Rytuba, J.J., Grubensky, M.J. and Goeldner, C.A., 1987a, Geologic map of the Bannock Ridge quadrangle, Malheur County, Oregon: U.S. Geological Survey Miscellaneous Field Studies Map MF-1903, scale 1:24,000. [Also available at https://pubs.er.usgs.gov/publication/mf1903.]

Vander Meulen, D.B., Rytuba, J.J., Minor, S.A. and Harwood, C.S., 1989, Preliminary geologic map of the Three Fingers Rock quadrangle, Malheur County, Oregon: U.S. Geological Survey Open-File Report 89-344, scale 1:24,000. [Also available at https://pubs.er.usgs.gov/publication/ofr89344.] 
Vander Meulen, D.B., Rytuba, J.J., Vercoutere, T.L. and Minor, S.A., 1987b, Geologic map of the Rooster Comb quadrangle, Malheur County, Oregon: U.S. Geological Survey Miscellaneous Field Studies Map MF-1902, scale 1:24,000. [Also available at $\mathrm{https://pubs.er.usgs.gov/publication/mf1902.]}$

Walker, G.P.L., 1983, Ignimbrite types and Ignimbrite Problems, in: M. F. Sheridan and F. Barberi (Eds.), Explosive Volcanism: Journal of Volcanology and Geothermal Research, v. 17, p. 65-88.

Webb, B.M., Streck, M.J., Ferns, M.L. and McIntosh, W.C., 2015, Stratigraphy and geochemistry of a large, hotspot-related rhyolite of the Pacific Northwest-The Littlefield Rhyolite, eastern Oregon [abs.]: American Geophysical Union Abstract, n. V31E-3065.

Webb, B.M., Streck, M.J., McIntosh, W.C. and Ferns, M.L., 2016, Flow units of the Littlefield Rhyolite, eastern Oregon, constraining age and storage sites of Grande Ronde Basalt magmas [abs.]: Geological Society of America Abstracts with Programs, v. 48, n. 6.

White, D.L. and Vallier, T.L., 1994, Geologic evolution of the Pittsbug Landing area, Snake River Canyon, Oregon and Idaho, in Vallier, T.L. and Brooks, H.C. (Eds.), Stratigraphy, 131 Physiography, and Mineral Resources of the Blue Mountains Region: U.S. Geological Survey Professional Paper 1439, p. 55-74.

Whitsun, N.W., 1988, Geochemical stratigraphy of the Dooley Rhyolite Breccia and Tertiary basalts in the Dooley Mountain quadrangle [M.S. Thesis], Portland, Portland State University, Oregon 122 pp.

Wolff, J.A., Ramos, F.C., Hart, G.L., Patterson, J.D. and Brandon, A.D., 2008, Columbia River flood basalts from a centralized crustal magmatic system: Nature Geoscience, v. 1, p. 177-180, doi:10.1038/ngeo124.

Wood, J.D., 1976, The geology of the Castle Rock area, Grant, Harney and Malheur counties, Oregon [MS thesis]: Portland, Portland State University, 123 pp.

Wooden, B.J.L., Kistler, R.W. and Tosdal, R.M., 1999, Strontium, lead, and oxygen isotopic data for granitoid and volcanic rocks from the Northern Great Basin and Sierra Nevada, California, Nevada and Utah: U.S. Geological Survey Open-File Report, 99-569, 20 pp.

Wyld, S.J. and Wright, J.E., 2001, New evidence for Cretaceous strikeslip faulting in the United States Cordillera and implications for terrane-displacement, deformation patterns, and plutonism: American Journal of Science, v. 301, p. 150-181. 
Zoback, M.L. and Thompson, G.A., 1978, Basin and Range rifting in northern Nevada: Clues from a mid-Miocene rift and its subsequent offsets: Geology, v. 6, n. 2, p. $111-116$.

Zoback, M.L.,McKee, E.H., Blakely, R.J. and Thompson, G.A., 1994, The northern Nevada rift: Regional tectono-magmatic relations and middle Miocene stress directions: Geological Society of America Bulletin, v. 106, p. 371-382. 


\section{APPENDIX A: UNIT 1 CHEMICAL DATA, STANDARD DEVIATIONS AND GPS COORDINATES}

Table A1. Unit 1 average feldspar end member component composition, standard deviations and GPS coordinates by sample.

\begin{tabular}{lccccccccc}
\hline \hline Sample & $\begin{array}{c}\text { Number } \\
\text { of } \\
\text { Crystals }\end{array}$ & An & Ab & Or & $\begin{array}{c}\text { An } \\
\text { Std. }\end{array}$ & $\begin{array}{c}\text { Al } \\
\text { Std. }\end{array}$ & $\begin{array}{c}\text { Or } \\
\text { Std. }\end{array}$ & Latitude & Longitude \\
\hline MS-10-23 & 6 & 10.4 & 81.0 & 8.6 & 0.4 & 0.3 & 0.4 & 43.8899 & -119.0325 \\
MS-10-23_xl5 & 1 & 14.0 & 79.7 & 6.2 & 0.5 & 0.1 & 0.6 & 43.8899 & -119.0325 \\
MS-11-4.1 & 5 & 10.7 & 81.1 & 8.3 & 0.8 & 0.3 & 0.7 & 44.0241 & -119.1875 \\
MS-11-4.1_x11 & 1 & 13.3 & 80.6 & 6.1 & 0.2 & 0.3 & 0.1 & 44.0241 & -119.1875 \\
MS-11-3 & 4 & 10.7 & 79.8 & 9.6 & 0.8 & 0.3 & 0.5 & 44.0871 & -119.2209 \\
MS-11-4.2 & 4 & 10.7 & 80.8 & 8.5 & 1.1 & 0.6 & 0.5 & 44.0269 & -119.1841 \\
MS-11-4.2_x12 & 1 & 14.2 & 79.4 & 6.4 & N/A & N/A & N/A & 44.0269 & -119.1841 \\
MS-12-23 & 7 & 11.9 & 78.5 & 9.6 & 0.8 & 0.4 & 0.6 & 44.5613 & -118.0251 \\
MS-15-7a & 7 & 11.1 & 80.3 & 8.6 & 0.8 & 0.8 & 0.2 & 43.7526 & -118.7989 \\
MS-15-38 & 6 & 10.6 & 80.7 & 8.7 & 0.5 & 0.3 & 0.3 & 44.0106 & -118.7819 \\
MS-15-32b & 7 & 10.8 & 81.8 & 7.4 & 0.5 & 0.3 & 0.5 & 44.0742 & -117.7852 \\
MS-15-34a & 6 & 10.9 & 80.7 & 8.4 & 1.3 & 0.4 & 1.0 & 44.7506 & -116.8918 \\
MS-12-30 & 9 & 11.8 & 80.0 & 8.2 & 2.2 & 1.0 & 1.3 & 44.0747 & -117.5385 \\
MS-14-4d & 10 & 10.4 & 81.2 & 8.5 & 1.0 & 0.6 & 0.6 & 43.5947 & -117.8269 \\
PFC-15486 & 10 & 10.9 & 80.8 & 8.3 & 1.7 & 1.0 & 1.1 & 44.0063 & -118.8330 \\
MS-14-22 & 1 & 11.0 & 80.3 & 8.7 & 0.8 & 0.4 & 0.5 & 44.1332 & -118.8781 \\
\hline
\end{tabular}


Table A2. Unit 1 EMP major element individual feldspar crystal chemical data.

\begin{tabular}{|c|c|c|c|c|c|c|c|c|c|}
\hline $\begin{array}{l}\text { Sample and } \\
\text { crystal } \\
\text { number** }\end{array}$ & $\mathrm{SiO}_{2}$ & $\mathrm{Al}_{2} \mathrm{O}_{3}$ & $\mathrm{FeO}^{*}$ & MgO & $\mathrm{CaO}$ & $\mathrm{Na}_{2} \mathrm{O}$ & $\mathrm{K}_{2} \mathrm{O}$ & $\mathrm{BaO}$ & Total \\
\hline \multicolumn{10}{|l|}{ MS-10-231 } \\
\hline xl_1a & 63.87 & 22.28 & 0.28 & 0.000 & 2.14 & 9.51 & 1.51 & 0.42 & 100.0 \\
\hline xl_1b & 64.24 & 22.11 & 0.25 & 0.000 & 2.16 & 9.30 & 1.53 & 0.41 & 100.0 \\
\hline $\mathrm{xl} \_2 \mathrm{a}$ & 64.08 & 22.14 & 0.29 & 0.001 & 2.09 & 9.48 & 1.48 & 0.44 & 100.0 \\
\hline$x l \_2 b$ & 63.98 & 22.34 & 0.27 & 0.000 & 2.29 & 9.38 & 1.36 & 0.37 & 100.0 \\
\hline xl_3a & 63.94 & 22.15 & 0.30 & 0.000 & 2.20 & 9.45 & 1.52 & 0.43 & 100.0 \\
\hline$x l \_3 b$ & 63.86 & 22.45 & 0.20 & 0.009 & 2.22 & 9.30 & 1.48 & 0.48 & 100.0 \\
\hline xl_4a & 64.15 & 22.10 & 0.25 & 0.000 & 2.25 & 9.35 & 1.48 & 0.42 & 100.0 \\
\hline $\mathrm{xl} \_4 \mathrm{~b}$ & 64.46 & 21.85 & 0.29 & 0.000 & 2.12 & 9.31 & 1.51 & 0.45 & 100.0 \\
\hline xl_5a & 63.29 & 22.83 & 0.30 & 0.003 & 3.01 & 9.28 & 1.03 & 0.26 & 100.0 \\
\hline xl_5b & 63.62 & 22.64 & 0.20 & 0.000 & 2.86 & 9.22 & 1.16 & 0.29 & 100.0 \\
\hline xl_6a & 64.15 & 22.03 & 0.35 & 0.030 & 2.15 & 9.25 & 1.61 & 0.44 & 100.0 \\
\hline xl_6b & 63.82 & 22.36 & 0.28 & 0.000 & 2.35 & 9.33 & 1.43 & 0.43 & 100.0 \\
\hline xl_7a & 64.67 & 21.86 & 0.31 & 0.000 & 2.00 & 9.12 & 1.56 & 0.48 & 100.0 \\
\hline $\mathrm{xl} \_7 \mathrm{~b}$ & 64.33 & 21.92 & 0.29 & 0.000 & 2.12 & 9.40 & 1.50 & 0.43 & 100.0 \\
\hline \multicolumn{10}{|l|}{ MS-11-4.1 ${ }^{1}$} \\
\hline xl_1a & 63.16 & 22.55 & 0.24 & 0.011 & 2.88 & 9.80 & 1.11 & 0.24 & 100.0 \\
\hline $\mathrm{xl} \_1 \mathrm{~b}$ & 63.49 & 22.53 & 0.34 & 0.000 & 2.84 & 9.42 & 1.11 & 0.27 & 100.0 \\
\hline $\mathrm{xl} \_2 \mathrm{a}$ & 64.30 & 21.99 & 0.22 & 0.000 & 2.05 & 9.31 & 1.63 & 0.49 & 100.0 \\
\hline $\mathrm{xl} \_2 \mathrm{~b}$ & 63.54 & 22.61 & 0.26 & 0.000 & 2.62 & 9.39 & 1.22 & 0.37 & 100.0 \\
\hline xl_4a & 64.28 & 22.03 & 0.21 & 0.001 & 2.16 & 9.37 & 1.53 & 0.43 & 100.0 \\
\hline xl_4b & 63.73 & 22.29 & 0.24 & 0.000 & 2.27 & 9.62 & 1.44 & 0.41 & 100.0 \\
\hline xl_5a & 63.81 & 22.53 & 0.23 & 0.000 & 2.33 & 9.34 & 1.36 & 0.41 & 100.0 \\
\hline xl_5b & 64.59 & 22.06 & 0.21 & 0.000 & 2.08 & 9.14 & 1.47 & 0.44 & 100.0 \\
\hline xl_6a & 63.95 & 22.05 & 0.30 & 0.000 & 2.11 & 9.52 & 1.58 & 0.48 & 100.0 \\
\hline xl_6b & 63.84 & 22.44 & 0.27 & 0.003 & 2.11 & 9.43 & 1.44 & 0.46 & 100.0 \\
\hline xl_8a & 63.55 & 22.69 & 0.28 & 0.000 & 2.23 & 9.45 & 1.41 & 0.39 & 100.0 \\
\hline $\mathrm{xl} \_8 \mathrm{~b}$ & 63.63 & 22.54 & 0.31 & 0.000 & 2.33 & 9.37 & 1.41 & 0.40 & 100.0 \\
\hline \multicolumn{10}{|l|}{ MS-11-3 } \\
\hline $\mathrm{xl} \_2$ & 64.25 & 21.50 & 0.23 & 0.002 & 2.26 & 8.91 & 1.33 & N/A & 98.5 \\
\hline xl_3 & 64.36 & 20.85 & 0.24 & 0.000 & 2.00 & 8.86 & 1.46 & N/A & 97.8 \\
\hline xl_4 & 63.07 & 21.81 & 0.32 & 0.000 & 2.31 & 8.81 & 1.29 & N/A & 97.6 \\
\hline xl_5 & 62.94 & 21.34 & 0.28 & 0.000 & 2.09 & 9.24 & 1.47 & N/A & 97.4 \\
\hline \multicolumn{10}{|l|}{ MS-11-4.2 } \\
\hline xl_1 & 64.83 & 21.14 & 0.24 & 0.006 & 2.12 & 8.77 & 1.43 & N/A & 98.5 \\
\hline xl_2 & 64.70 & 21.92 & 0.24 & 0.003 & 2.88 & 8.93 & 1.08 & N/A & 99.7 \\
\hline xl_3 & 64.48 & 21.50 & 0.27 & 0.000 & 2.32 & 8.91 & 1.32 & N/A & 98.8 \\
\hline xl_4 & 65.48 & 20.90 & 0.28 & 0.000 & 1.86 & 9.17 & 1.56 & N/A & 99.2 \\
\hline
\end{tabular}


Table A2 continued.

\begin{tabular}{|c|c|c|c|c|c|c|c|c|c|}
\hline $\begin{array}{l}\text { Sample and } \\
\text { crystal } \\
\text { number** }\end{array}$ & $\mathrm{SiO}_{2}$ & $\mathrm{Al}_{2} \mathrm{O}_{3}$ & $\mathrm{FeO}^{*}$ & MgO & $\mathrm{CaO}$ & $\mathrm{Na}_{2} \mathrm{O}$ & $\mathrm{K}_{2} \mathrm{O}$ & $\mathrm{BaO}$ & Total \\
\hline \multicolumn{10}{|l|}{$\begin{array}{l}\text { MS-11-4.2 } \\
\text { cont. }\end{array}$} \\
\hline xl_5 & 66.96 & 21.33 & 0.21 & 0.000 & 2.24 & 8.82 & 1.39 & N/A & 100.9 \\
\hline \multicolumn{10}{|l|}{ MS-12-23 } \\
\hline xl_1a & 67.65 & 21.71 & 0.26 & 0.000 & 1.96 & 9.08 & 1.56 & 0.48 & 102.7 \\
\hline $\mathrm{xl} \_1 \mathrm{~b}$ & 67.40 & 21.35 & 0.24 & 0.014 & 2.11 & 8.95 & 1.48 & 0.51 & 102.0 \\
\hline $\mathrm{xl} \_2 \mathrm{a}$ & 67.37 & 21.51 & 0.31 & 0.004 & 2.18 & 9.07 & 1.42 & 0.47 & 102.3 \\
\hline $\mathrm{xl} \_2 \mathrm{~b}$ & 66.96 & 21.65 & 0.26 & 0.008 & 2.35 & 9.01 & 1.32 & 0.42 & 102.0 \\
\hline xl_3a & 67.34 & 21.39 & 0.24 & 0.000 & 2.07 & 8.84 & 1.48 & 0.52 & 101.9 \\
\hline $\mathrm{xl} \_3 \mathrm{~b}$ & 66.41 & 21.64 & 0.23 & 0.000 & 2.65 & 9.08 & 1.22 & 0.42 & 101.6 \\
\hline$x l \_4 a$ & 67.77 & 21.26 & 0.29 & 0.000 & 2.10 & 8.73 & 1.46 & 0.43 & 102.0 \\
\hline$x l \_4 b$ & 67.94 & 21.49 & 0.21 & 0.008 & 2.12 & 8.90 & 1.53 & 0.49 & 102.7 \\
\hline xl_5a & 67.36 & 21.80 & 0.23 & 0.000 & 2.13 & 8.86 & 1.42 & 0.47 & 102.3 \\
\hline $\mathrm{xl} \_5 \mathrm{~b}$ & 67.53 & 21.18 & 0.24 & 0.000 & 1.98 & 8.88 & 1.57 & 0.57 & 101.9 \\
\hline xl_6a & 68.48 & 21.51 & 0.25 & 0.004 & 1.95 & 9.04 & 1.41 & 0.44 & 103.1 \\
\hline xl_6b & 68.23 & 21.02 & 0.24 & 0.000 & 2.04 & 8.93 & 1.49 & 0.50 & 102.4 \\
\hline xl_7a & 67.20 & 21.87 & 0.27 & 0.003 & 2.17 & 8.92 & 1.46 & 0.50 & 102.4 \\
\hline xl_7b & 66.74 & 21.59 & 0.23 & 0.000 & 2.14 & 9.05 & 1.44 & 0.44 & 101.6 \\
\hline \multicolumn{10}{|l|}{ MS-15-7a } \\
\hline xl_1 & 66.40 & 21.55 & 0.25 & 0.000 & 2.28 & 8.32 & 1.35 & N/A & 100.2 \\
\hline xl_2 & 67.88 & 21.40 & 0.28 & 0.000 & 2.03 & 8.93 & 1.42 & N/A & 101.9 \\
\hline xl_3 & 66.36 & 21.57 & 0.25 & 0.000 & 2.25 & 8.48 & 1.39 & N/A & 100.3 \\
\hline xl_4 & 66.69 & 21.46 & 0.22 & 0.000 & 2.11 & 9.13 & 1.42 & N/A & 101.0 \\
\hline xl_5 & 66.31 & 21.64 & 0.24 & 0.000 & 2.31 & 8.53 & 1.41 & N/A & 100.4 \\
\hline xl_6 & 67.28 & 21.35 & 0.27 & 0.000 & 2.03 & 8.85 & 1.45 & N/A & 101.2 \\
\hline xl_7 & 66.58 & 21.32 & 0.23 & 0.000 & 2.09 & 8.49 & 1.41 & N/A & 100.1 \\
\hline \multicolumn{10}{|l|}{ MS-15-38 } \\
\hline xl_1 & 66.30 & 20.82 & 0.26 & 0.001 & 1.88 & 8.67 & 1.50 & N/A & 99.4 \\
\hline$x l \_2$ & 66.54 & 21.18 & 0.25 & 0.002 & 2.20 & 8.80 & 1.44 & N/A & 100.4 \\
\hline$x l \_3$ & 65.74 & 20.79 & 0.23 & 0.000 & 2.05 & 8.59 & 1.37 & N/A & 98.8 \\
\hline xl_4 & 66.56 & 20.66 & 0.27 & 0.000 & 2.09 & 8.75 & 1.45 & N/A & 99.8 \\
\hline xl_6 & 66.76 & 20.52 & 0.25 & 0.000 & 2.19 & 9.11 & 1.37 & N/A & 100.2 \\
\hline xl_7 & 66.67 & 20.79 & 0.29 & 0.000 & 2.08 & 8.78 & 1.45 & N/A & 100.1 \\
\hline \multicolumn{10}{|l|}{ MS-15-32b } \\
\hline xl_1 & 66.27 & 20.97 & 0.15 & 0.000 & 2.13 & 8.93 & 1.40 & N/A & 99.9 \\
\hline$x l \_2$ & 66.88 & 21.76 & 0.25 & 0.000 & 2.25 & 9.24 & 1.29 & N/A & 101.7 \\
\hline xl_3 & 66.53 & 20.95 & 0.26 & 0.000 & 2.18 & 8.72 & 1.37 & N/A & 100.0 \\
\hline xl_4 & 66.38 & 21.32 & 0.26 & 0.000 & 2.26 & 9.02 & 1.32 & N/A & 100.6 \\
\hline
\end{tabular}


Table A2 continued.

\begin{tabular}{|c|c|c|c|c|c|c|c|c|c|}
\hline $\begin{array}{l}\text { Sample and } \\
\text { crystal } \\
\text { number** }\end{array}$ & $\mathrm{SiO}_{2}$ & $\mathrm{Al}_{2} \mathrm{O}_{3}$ & $\mathrm{FeO}^{*}$ & MgO & $\mathrm{CaO}$ & $\mathrm{Na}_{2} \mathrm{O}$ & $\mathrm{K}_{2} \mathrm{O}$ & $\mathrm{BaO}$ & Total \\
\hline \multicolumn{10}{|l|}{$\begin{array}{l}\text { MS-15-32b } \\
\text { cont. }\end{array}$} \\
\hline $\mathrm{xl} \_5$ & 66.28 & 20.47 & 0.16 & 0.000 & 1.91 & 8.76 & 1.46 & N/A & 99.0 \\
\hline xl_6 & 65.56 & 20.75 & 0.19 & 0.002 & 2.10 & 8.80 & 1.37 & N/A & 98.8 \\
\hline xl_7 & 66.55 & 20.66 & 0.26 & 0.000 & 2.08 & 8.94 & 1.50 & N/A & 100.0 \\
\hline \multicolumn{10}{|l|}{ MS-15-34a } \\
\hline xl_1 & 65.70 & 21.07 & 0.22 & 0.000 & 2.13 & 8.61 & 1.38 & N/A & 99.9 \\
\hline $\mathrm{xl} \_2$ & 65.55 & 20.96 & 0.28 & 0.002 & 2.10 & 8.69 & 1.37 & N/A & 101.7 \\
\hline xl_4 & 66.27 & 20.58 & 0.23 & 0.006 & 1.85 & 8.95 & 1.55 & N/A & 100.6 \\
\hline xl_5 & 65.14 & 21.27 & 0.21 & 0.000 & 2.26 & 8.79 & 1.34 & N/A & 99.0 \\
\hline xl_6 & 64.25 & 20.64 & 0.25 & 0.001 & 1.91 & 8.76 & 1.54 & N/A & 98.8 \\
\hline xl_7 & 64.35 & 21.75 & 0.30 & 0.004 & 2.55 & 8.85 & 1.12 & N/A & 100.0 \\
\hline \multicolumn{10}{|l|}{ MS-12-30 } \\
\hline $\mathrm{xl} \_1 \mathrm{a}$ & 66.35 & 21.33 & 0.23 & 0.001 & 1.97 & 8.73 & 1.55 & 0.49 & 100.7 \\
\hline $\mathrm{xl} \_1 \mathrm{~b}$ & 66.64 & 21.38 & 0.25 & 0.000 & 1.94 & 8.70 & 1.57 & 0.44 & 100.9 \\
\hline $\mathrm{xl} \_2 \mathrm{a}$ & 66.25 & 21.82 & 0.20 & 0.003 & 2.53 & 8.66 & 1.26 & 0.37 & 101.1 \\
\hline $\mathrm{xl} \_2 \mathrm{~b}$ & 66.09 & 21.39 & 0.29 & 0.003 & 2.02 & 8.68 & 1.52 & 0.43 & 100.4 \\
\hline xl_3a & 64.72 & 22.10 & 0.26 & 0.010 & 3.04 & 8.54 & 1.03 & 0.32 & 100.0 \\
\hline$x l \_3 b$ & 65.37 & 22.01 & 0.22 & 0.000 & 2.78 & 8.60 & 1.10 & 0.32 & 100.4 \\
\hline xl_4a & 66.80 & 21.20 & 0.28 & 0.006 & 2.04 & 8.87 & 1.51 & 0.45 & 101.2 \\
\hline $\mathrm{xl} \_4 \mathrm{~b}$ & 66.36 & 21.07 & 0.22 & 0.000 & 1.81 & 9.00 & 1.70 & 0.53 & 100.7 \\
\hline xl_5a & 66.88 & 21.41 & 0.19 & 0.001 & 2.14 & 8.67 & 1.46 & 0.49 & 101.2 \\
\hline $\mathrm{xl} \_5 \mathrm{~b}$ & 65.56 & 21.60 & 0.22 & 0.000 & 2.07 & 8.50 & 1.43 & 0.49 & 99.9 \\
\hline xl_6a & 64.83 & 22.54 & 0.22 & 0.000 & 3.31 & 8.54 & 0.94 & 0.25 & 100.6 \\
\hline xl_6b & 65.90 & 21.45 & 0.24 & 0.002 & 2.29 & 8.57 & 1.36 & 0.43 & 100.2 \\
\hline xl_7a & 64.68 & 22.73 & 0.31 & 0.003 & 3.18 & 8.91 & 1.01 & 0.31 & 101.1 \\
\hline xl_7b & 65.18 & 21.70 & 0.28 & 0.000 & 2.36 & 8.82 & 1.25 & 0.41 & 100.0 \\
\hline xl_8a & 65.12 & 21.55 & 0.21 & 0.000 & 2.21 & 8.61 & 1.38 & 0.42 & 99.5 \\
\hline $\mathrm{xl} \_8 \mathrm{~b}$ & 66.55 & 21.45 & 0.24 & 0.000 & 2.04 & 9.04 & 1.43 & 0.43 & 101.2 \\
\hline xl_9a & 65.98 & 21.69 & 0.25 & 0.004 & 2.12 & 8.93 & 1.43 & 0.46 & 100.9 \\
\hline xl_9b & 64.46 & 21.38 & 0.24 & 0.001 & 2.09 & 8.81 & 1.45 & 0.45 & 98.9 \\
\hline \multicolumn{10}{|l|}{ MS-14-4d } \\
\hline $\mathrm{xl} \_1 \mathrm{a}$ & 66.03 & 21.97 & 0.25 & 0.000 & 2.36 & 8.74 & 1.35 & 0.43 & 101.1 \\
\hline $\mathrm{xl} \_1 \mathrm{~b}$ & 65.61 & 21.41 & 0.24 & 0.000 & 2.18 & 8.88 & 1.39 & 0.39 & 100.1 \\
\hline $\mathrm{xl} \_2 \mathrm{a}$ & 65.92 & 21.92 & 0.23 & 0.000 & 2.48 & 8.77 & 1.28 & 0.43 & 101.0 \\
\hline $\mathrm{xl} \_2 \mathrm{~b}$ & 66.55 & 21.89 & 0.29 & 0.000 & 2.18 & 9.05 & 1.37 & 0.42 & 101.8 \\
\hline xl_3a & 65.60 & 21.67 & 0.28 & 0.005 & 2.21 & 8.98 & 1.39 & 0.46 & 100.6 \\
\hline$x l \_3 b$ & 66.32 & 21.64 & 0.27 & 0.001 & 2.18 & 8.88 & 1.40 & 0.49 & 101.2 \\
\hline
\end{tabular}


Table A2 continued.

\begin{tabular}{|c|c|c|c|c|c|c|c|c|c|}
\hline $\begin{array}{l}\text { Sample and } \\
\text { crystal } \\
\text { number** }\end{array}$ & $\mathrm{SiO}_{2}$ & $\mathrm{Al}_{2} \mathrm{O}_{3}$ & $\mathrm{FeO}^{*}$ & MgO & $\mathrm{CaO}$ & $\mathrm{Na}_{2} \mathrm{O}$ & $\mathbf{K}_{2} \mathbf{O}$ & $\mathrm{BaO}$ & Total \\
\hline \multicolumn{10}{|l|}{$\begin{array}{l}\text { MS-14-4d } \\
\text { cont. }\end{array}$} \\
\hline xl_4a & 66.28 & 21.27 & 0.26 & 0.002 & 2.05 & 8.82 & 1.45 & 0.41 & 100.5 \\
\hline $\mathrm{xl} \_4 \mathrm{~b}$ & 64.92 & 21.54 & 0.24 & 0.000 & 2.44 & 8.64 & 1.39 & 0.42 & 99.6 \\
\hline xl_5a & 65.78 & 21.52 & 0.22 & 0.010 & 2.36 & 8.79 & 1.30 & 0.44 & 100.4 \\
\hline xl_5b & 65.36 & 21.35 & 0.34 & 0.000 & 2.12 & 8.77 & 1.47 & 0.45 & 99.9 \\
\hline xl_6a & 65.40 & 21.24 & 0.21 & 0.000 & 2.07 & 8.81 & 1.48 & 0.47 & 99.7 \\
\hline xl_6b & 65.62 & 21.59 & 0.20 & 0.000 & 2.03 & 8.83 & 1.47 & 0.43 & 100.2 \\
\hline xl_7a & 65.73 & 21.61 & 0.20 & 0.000 & 2.21 & 8.86 & 1.41 & 0.44 & 100.4 \\
\hline xl_7b & 65.50 & 21.08 & 0.22 & 0.000 & 1.96 & 8.71 & 1.60 & 0.47 & 99.5 \\
\hline xl_8a & 66.09 & 21.26 & 0.25 & 0.000 & 2.12 & 8.72 & 1.49 & 0.48 & 100.4 \\
\hline $\mathrm{xl} \_8 \mathrm{~b}$ & 65.10 & 21.81 & 0.25 & 0.000 & 2.29 & 9.19 & 1.36 & 0.44 & 100.4 \\
\hline xl_9a & 63.55 & 21.90 & 0.18 & 0.013 & 2.78 & 8.47 & 1.08 & 0.40 & 98.4 \\
\hline xl_9b & 65.64 & 21.30 & 0.23 & 0.000 & 2.04 & 8.81 & 1.42 & 0.40 & 99.8 \\
\hline xl_10a & 64.72 & 21.90 & 0.30 & 0.000 & 2.35 & 8.71 & 1.42 & 0.44 & 99.8 \\
\hline xl_10b & 65.43 & 21.40 & 0.21 & 0.004 & 2.22 & 8.76 & 1.33 & 0.42 & 99.8 \\
\hline \multicolumn{10}{|l|}{ PFC-15486 } \\
\hline xl_1a & 65.85 & 21.16 & 0.25 & 0.005 & 1.97 & 8.73 & 1.49 & 0.56 & 100.0 \\
\hline $\mathrm{xl} \_1 \mathrm{~b}$ & 66.52 & 21.15 & 0.21 & 0.000 & 2.08 & 8.78 & 1.52 & 0.45 & 100.7 \\
\hline xl_2a & 65.44 & 22.12 & 0.30 & 0.000 & 3.25 & 8.57 & 0.97 & 0.27 & 100.9 \\
\hline xl_2b & 64.76 & 21.91 & 0.27 & 0.000 & 3.00 & 8.80 & 0.99 & 0.29 & 100.0 \\
\hline xl_3a & 65.21 & 21.81 & 0.30 & 0.009 & 2.31 & 8.65 & 1.40 & 0.40 & 100.1 \\
\hline$x l \_3 b$ & 60.95 & 19.96 & 1.56 & 0.177 & 2.25 & 7.09 & 1.30 & 0.39 & 93.7 \\
\hline xl_4a & 65.77 & 20.78 & 0.29 & 0.000 & 1.99 & 8.75 & 1.52 & 0.50 & 99.6 \\
\hline xl_4b & 66.59 & 21.22 & 0.26 & 0.002 & 2.03 & 9.13 & 1.54 & 0.51 & 101.3 \\
\hline xl_5a & 65.58 & 21.18 & 0.25 & 0.006 & 2.25 & 8.92 & 1.40 & 0.43 & 100.0 \\
\hline xl_5b & 65.15 & 20.56 & 0.29 & 0.007 & 2.08 & 8.73 & 1.44 & 0.48 & 98.7 \\
\hline xl_6a & 65.34 & 21.27 & 0.19 & 0.000 & 2.16 & 8.56 & 1.37 & 0.47 & 99.4 \\
\hline xl_6b & 66.15 & 21.37 & 0.22 & 0.000 & 2.30 & 8.85 & 1.34 & 0.39 & 100.6 \\
\hline xl_7a & 65.50 & 21.22 & 0.24 & 0.000 & 2.43 & 8.98 & 1.33 & 0.42 & 100.1 \\
\hline xl_7b & 65.92 & 20.80 & 0.27 & 0.000 & 2.26 & 8.60 & 1.43 & 0.42 & 99.7 \\
\hline xl_8a & 65.66 & 21.56 & 0.26 & 0.000 & 2.49 & 8.90 & 1.26 & 0.41 & 100.5 \\
\hline $\mathrm{xl} \_8 \mathrm{~b}$ & 64.18 & 21.65 & 0.30 & 0.005 & 2.84 & 8.69 & 1.11 & 0.36 & 99.1 \\
\hline xl_9a & 65.22 & 21.44 & 0.30 & 0.000 & 2.32 & 8.91 & 1.41 & 0.44 & 100.0 \\
\hline xl_9b & 65.25 & 21.30 & 0.20 & 0.000 & 2.22 & 8.62 & 1.40 & 0.43 & 99.4 \\
\hline xl_10a & 65.87 & 20.85 & 0.29 & 0.000 & 1.99 & 8.72 & 1.52 & 0.47 & 99.7 \\
\hline xl_10b & 66.17 & 21.48 & 0.27 & 0.000 & 2.24 & 8.75 & 1.34 & 0.46 & 100.7 \\
\hline
\end{tabular}


Table A2 continued.

\begin{tabular}{|c|c|c|c|c|c|c|c|c|c|}
\hline $\begin{array}{l}\text { Sample and } \\
\text { crystal } \\
\text { number** }\end{array}$ & $\mathrm{SiO}_{2}$ & $\mathrm{Al}_{2} \mathrm{O}_{3}$ & $\mathrm{FeO}^{*}$ & MgO & $\mathrm{CaO}$ & $\mathrm{Na}_{2} \mathrm{O}$ & $\mathrm{K}_{2} \mathrm{O}$ & $\mathrm{BaO}$ & Total \\
\hline \multicolumn{10}{|l|}{ MS-14-22 } \\
\hline $\mathrm{xl} \_1 \mathrm{a}$ & 65.34 & 21.20 & 0.22 & 0.000 & 2.31 & 8.78 & 1.39 & 0.43 & 99.7 \\
\hline xl_1b & 65.56 & 21.36 & 0.27 & 0.000 & 2.32 & 9.00 & 1.40 & 0.51 & 100.4 \\
\hline $\mathrm{xl} \_1 \mathrm{c}$ & 65.45 & 21.02 & 0.27 & 0.000 & 2.19 & 8.96 & 1.55 & 0.48 & 99.9 \\
\hline$x 1 \_1 d$ & 65.83 & 21.38 & 0.25 & 0.000 & 2.00 & 9.02 & 1.54 & 0.48 & 100.5 \\
\hline
\end{tabular}

** Letters following crystal number indicate multiple analysis points per crystal.

1. Normalized values. 
Table A3. Unit 1 individual feldspar crystal end member compositions.

\begin{tabular}{|c|c|c|c|}
\hline Sample and crystal number** & $A n^{2}$ & $\mathbf{A} \mathbf{b}^{3}$ & $\mathrm{Or}^{4}$ \\
\hline \multicolumn{4}{|l|}{ MS-10-23 ${ }^{1}$} \\
\hline $\mathrm{xl} \_1 \mathrm{a}$ & 10.15 & 81.33 & 8.53 \\
\hline $\mathrm{xl} \_1 \mathrm{~b}$ & 10.40 & 80.81 & 8.80 \\
\hline $\mathrm{xl} \_2 \mathrm{a}$ & 9.97 & 81.58 & 8.45 \\
\hline $\mathrm{xl} \_2 \mathrm{~b}$ & 11.02 & 81.21 & 7.78 \\
\hline $\mathrm{xl} \_3 \mathrm{a}$ & 10.46 & 80.95 & 8.60 \\
\hline $\mathrm{xl} \_3 \mathrm{~b}$ & 10.72 & 80.78 & 8.51 \\
\hline xl_4a & 10.78 & 80.77 & 8.45 \\
\hline $\mathrm{xl} \_4 \mathrm{~b}$ & 10.22 & 81.09 & 8.71 \\
\hline $\mathrm{xl} \_5 \mathrm{a}$ & 14.37 & 79.79 & 5.84 \\
\hline $\mathrm{xl} \_5 \mathrm{~b}$ & 13.70 & 79.67 & 6.63 \\
\hline xl_6a & 10.38 & 80.40 & 9.23 \\
\hline xl_6b & 11.25 & 80.58 & 8.18 \\
\hline $\mathrm{xl} \_7 \mathrm{a}$ & 9.88 & 80.99 & 9.14 \\
\hline $\mathrm{xl} \_7 \mathrm{~b}$ & 10.17 & 81.26 & 8.58 \\
\hline \multicolumn{4}{|l|}{ MS-11-4.1 ${ }^{1}$} \\
\hline xl_1a & 13.15 & 80.81 & 6.04 \\
\hline $\mathrm{xl} \_1 \mathrm{~b}$ & 13.45 & 80.32 & 6.24 \\
\hline $\mathrm{xl} \_2 \mathrm{a}$ & 9.89 & 80.79 & 9.34 \\
\hline $\mathrm{xl} \_2 \mathrm{~b}$ & 12.46 & 80.61 & 6.94 \\
\hline xl_4a & 10.35 & 80.92 & 8.73 \\
\hline$x l \_4 b$ & 10.63 & 81.32 & 8.06 \\
\hline xl_5a & 11.20 & 81.02 & 7.79 \\
\hline $\mathrm{xl} \_5 \mathrm{~b}$ & 10.26 & 81.12 & 8.62 \\
\hline xl_6a & 9.97 & 81.13 & 8.90 \\
\hline xl_6b & 10.15 & 81.61 & 8.25 \\
\hline $\mathrm{xl} \_8 \mathrm{a}$ & 10.63 & 81.33 & 8.05 \\
\hline $\mathrm{xl} \_8 \mathrm{~b}$ & 11.16 & 80.80 & 8.05 \\
\hline \multicolumn{4}{|l|}{ MS-11-3 } \\
\hline xl_2 & 11.36 & 80.66 & 7.98 \\
\hline xl_3 & 10.15 & 81.00 & 8.85 \\
\hline xl_4 & 11.69 & 80.51 & 7.80 \\
\hline xl_5 & 10.21 & 81.25 & 8.55 \\
\hline \multicolumn{4}{|l|}{ MS-11-4.2 } \\
\hline xl_1 & 10.81 & 80.54 & 8.66 \\
\hline xl_2 & 14.20 & 79.45 & 6.35 \\
\hline xl_3 & 11.63 & 80.47 & 7.90 \\
\hline xl_4 & 9.18 & 81.65 & 9.19 \\
\hline xl_5 & 11.31 & 80.35 & 8.35 \\
\hline
\end{tabular}


Table A3 continued.

\begin{tabular}{|c|c|c|c|}
\hline Sample and crystal number** & $A n^{2}$ & $\mathbf{A b ^ { 3 }}$ & $\mathrm{Or}^{4}$ \\
\hline \multicolumn{4}{|l|}{ MS-12-23 } \\
\hline $\mathrm{xl} \_1 \mathrm{a}$ & 9.69 & 81.11 & 9.21 \\
\hline $\mathrm{xl} \_1 \mathrm{~b}$ & 10.56 & 80.62 & 8.83 \\
\hline $\mathrm{xl} \_2 \mathrm{a}$ & 10.76 & 80.87 & 8.38 \\
\hline $\mathrm{xl} \_2 \mathrm{~b}$ & 11.66 & 80.53 & 7.82 \\
\hline$x l \_3 a$ & 10.46 & 80.64 & 8.90 \\
\hline $\mathrm{xl} \_3 \mathrm{~b}$ & 12.95 & 79.96 & 7.10 \\
\hline xl_4a & 10.72 & 80.41 & 8.88 \\
\hline $\mathrm{xl} \_4 \mathrm{~b}$ & 10.62 & 80.26 & 9.14 \\
\hline xl_5a & 10.75 & 80.68 & 8.57 \\
\hline $\mathrm{xl} \_5 \mathrm{~b}$ & 9.95 & 80.65 & 9.41 \\
\hline xl_6a & 9.78 & 81.77 & 8.45 \\
\hline xl_6b & 10.23 & 80.83 & 8.95 \\
\hline $\mathrm{xl} \_7 \mathrm{a}$ & 10.85 & 80.47 & 8.69 \\
\hline xl_7b & 10.62 & 80.88 & 8.51 \\
\hline \multicolumn{4}{|l|}{ MS-15-7a } \\
\hline $\mathrm{xl} \_1$ & 12.09 & 79.38 & 8.54 \\
\hline xl_2 & 10.23 & 81.21 & 8.57 \\
\hline xl_3 & 11.71 & 79.66 & 8.64 \\
\hline xl_4 & 10.41 & 81.24 & 8.36 \\
\hline xl_5 & 11.91 & 79.41 & 8.68 \\
\hline xl_6 & 10.31 & 80.93 & 8.77 \\
\hline xl_7 & 10.94 & 80.26 & 8.81 \\
\hline \multicolumn{4}{|l|}{ MS-15-38 } \\
\hline xl_1 & 9.76 & 80.96 & 9.29 \\
\hline $\mathrm{xl} \_2$ & 11.13 & 80.22 & 8.66 \\
\hline xl_3 & 10.68 & 80.79 & 8.54 \\
\hline xl_4 & 10.66 & 80.50 & 8.85 \\
\hline xl_6 & 10.82 & 81.13 & 8.06 \\
\hline xl_7 & 10.61 & 80.59 & 8.81 \\
\hline \multicolumn{4}{|l|}{ MS-15-32b } \\
\hline $\mathrm{xl} \_1$ & 10.72 & 80.89 & 8.40 \\
\hline xl_2 & 10.99 & 81.47 & 7.55 \\
\hline xl_3 & 11.17 & 80.46 & 8.37 \\
\hline xl_4 & 11.27 & 80.93 & 7.81 \\
\hline xl_5 & 9.81 & 81.24 & 8.96 \\
\hline xl_6 & 10.71 & 80.99 & 8.31 \\
\hline xl_7 & 10.40 & 80.66 & 8.95 \\
\hline \multicolumn{4}{|l|}{ MS-15-34a } \\
\hline xl_1 & 11.04 & 80.44 & 8.53 \\
\hline
\end{tabular}


Table A3 continued.

\begin{tabular}{|c|c|c|c|}
\hline Sample and crystal number** & $\mathbf{A n ^ { 2 }}$ & $\mathbf{A b ^ { 3 }}$ & $\mathrm{Or}^{4}$ \\
\hline \multicolumn{4}{|l|}{ MS-15-34a cont. } \\
\hline xl_2 & 10.83 & 80.73 & 8.45 \\
\hline xl_4 & 9.33 & 81.37 & 9.30 \\
\hline xl_5 & 11.49 & 80.44 & 8.09 \\
\hline xl_6 & 9.78 & 80.84 & 9.39 \\
\hline xl_7 & 12.86 & 80.39 & 6.76 \\
\hline \multicolumn{4}{|l|}{ MS-12-30 } \\
\hline xl_1a & 10.05 & 80.48 & 9.47 \\
\hline $\mathrm{xl} \_1 \mathrm{~b}$ & 9.96 & 80.46 & 9.60 \\
\hline $\mathrm{xl} \_2 \mathrm{a}$ & 12.87 & 79.47 & 7.66 \\
\hline $\mathrm{xl} \_2 \mathrm{~b}$ & 10.38 & 80.34 & 9.29 \\
\hline xl_3a & 15.46 & 78.29 & 6.26 \\
\hline $\mathrm{xl} \_3 \mathrm{~b}$ & 14.20 & 79.14 & 6.67 \\
\hline $\mathrm{xl} \_4 \mathrm{a}$ & 10.31 & 80.65 & 9.05 \\
\hline $\mathrm{xl} \_4 \mathrm{~b}$ & 9.05 & 80.84 & 10.13 \\
\hline $\mathrm{xl} \_5 \mathrm{a}$ & 10.99 & 80.10 & 8.93 \\
\hline $\mathrm{xl} \_5 \mathrm{~b}$ & 10.82 & 80.24 & 8.95 \\
\hline xl_6a & 16.68 & 77.66 & 5.66 \\
\hline xl_6b & 11.81 & 79.84 & 8.36 \\
\hline xl_7a & 15.54 & 78.60 & 5.87 \\
\hline $\mathrm{xl} \_7 \mathrm{~b}$ & 11.95 & 80.53 & 7.53 \\
\hline xl_8a & 11.41 & 80.13 & 8.47 \\
\hline $\mathrm{xl} \_8 \mathrm{~b}$ & 10.17 & 81.35 & 8.49 \\
\hline xl_9a & 10.63 & 80.83 & 8.55 \\
\hline xl_9b & 10.62 & 80.62 & 8.77 \\
\hline \multicolumn{4}{|l|}{ MS-14-4d } \\
\hline $\mathrm{xl} \_1 \mathrm{a}$ & 11.97 & 79.87 & 8.17 \\
\hline$x l \_1 b$ & 10.97 & 80.66 & 8.38 \\
\hline $\mathrm{xl} \_2 \mathrm{a}$ & 12.54 & 79.76 & 7.71 \\
\hline $\mathrm{xl} \_2 \mathrm{~b}$ & 10.85 & 81.05 & 8.11 \\
\hline xl_3a & 11.02 & 80.71 & 8.28 \\
\hline $\mathrm{xl} \_3 \mathrm{~b}$ & 11.00 & 80.62 & 8.39 \\
\hline xl_4a & 10.41 & 80.82 & 8.78 \\
\hline $\mathrm{xl} \_4 \mathrm{~b}$ & 12.39 & 79.18 & 8.44 \\
\hline xl_5a & 11.93 & 80.20 & 7.88 \\
\hline $\mathrm{xl} \_5 \mathrm{~b}$ & 10.80 & 80.32 & 8.89 \\
\hline xl_6a & 10.51 & 80.57 & 8.93 \\
\hline xl_6b & 10.33 & 80.81 & 8.87 \\
\hline xl_7a & 11.11 & 80.46 & 8.44 \\
\hline xl_7b & 10.00 & 80.27 & 9.74 \\
\hline
\end{tabular}


Table A3 continued.

\begin{tabular}{cccc}
\hline \hline Sample and crystal number** & $\mathbf{A n}^{\mathbf{2}}$ & $\mathbf{A b}^{\mathbf{3}}$ & $\mathbf{O r}^{\mathbf{4}}$ \\
\hline MS-14-4d cont. & & & \\
\hline xl_8a & 10.82 & 80.16 & 9.04 \\
xl_8b & 11.19 & 80.88 & 7.94 \\
xl_9a & 14.40 & 78.97 & 6.64 \\
xl_9b & 10.39 & 80.97 & 8.65 \\
xl_10a & 11.88 & 79.52 & 8.60 \\
xl_10b & 11.35 & 80.59 & 8.07 \\
PFC-15486 & & & \\
\hline xl_1a & 10.11 & 80.78 & 9.12 \\
xl_1b & 10.53 & 80.31 & 9.17 \\
xl_2a & 16.39 & 77.78 & 5.83 \\
xl_2b & 14.96 & 79.15 & 5.89 \\
xl_3a & 11.81 & 79.69 & 8.51 \\
xl_3b & 13.58 & 77.08 & 9.35 \\
xl_4a & 10.19 & 80.57 & 9.25 \\
xl_4b & 9.99 & 80.98 & 9.03 \\
xl_5a & 11.26 & 80.42 & 8.33 \\
xl_5b & 10.64 & 80.59 & 8.78 \\
xl_6a & 11.25 & 80.25 & 8.52 \\
xl_6b & 11.59 & 80.38 & 8.03 \\
xl_7a & 12.02 & 80.16 & 7.83 \\
xl_7b & 11.59 & 79.66 & 8.76 \\
xl_8a & 12.45 & 80.07 & 7.49 \\
xl_8b & 14.34 & 79.00 & 6.66 \\
xl_9a & 11.58 & 80.08 & 8.36 \\
xl_9b & 11.43 & 79.97 & 8.61 \\
xl_10a & 10.20 & 80.50 & 9.31 \\
xl_10b & 11.42 & 80.42 & 8.17 \\
MS-14-22 & 11.50 & & \\
\hline xl_1a & 9.96 & 80.89 & 9.15 \\
xl_1b & & & \\
xl_1c & & & \\
xl_1d & & & \\
\hline
\end{tabular}

** Letters following crystal number indicate multiple analysis points per crystal.

1. Normalized values.

2. $\mathrm{An}=(\mathrm{CaO} * 0.714 / 40) /\left(\mathrm{CaO} * 0.714 / 40+\mathrm{Na}_{2} \mathrm{O} * 0.74 / 23+\mathrm{K}_{2} \mathrm{O} * 0.83 / 39\right) * 100$

3. $\mathrm{Ab}=\left(\mathrm{Na}_{2} \mathrm{O} * 0.74 / 23\right) /\left(\mathrm{CaO} * 0.714 / 40+\mathrm{Na}_{2} \mathrm{O} * 0.74 / 23+\mathrm{K}_{2} \mathrm{O} * 0.830 / 39\right) * 100$

4. $\mathrm{Or}=\left(\mathrm{K}_{2} \mathrm{O} * 0.83 / 39\right) /\left(\mathrm{CaO} * 0.714 / 40+\mathrm{Na}_{2} \mathrm{O} * 0.74 / 23+\mathrm{K}_{2} \mathrm{O} * 0.83 / 39\right) * 100$ 


\section{APPENDIX B: UNIT 2 CHEMICAL DATA, STANDARD DEVIATIONS AND GPS COORDINATES}

Table B1. Unit 2 average end member component composition and GPS coordinates by sample.

\begin{tabular}{lccccccccc}
\hline \hline Sample & $\begin{array}{c}\text { Number } \\
\text { of } \\
\text { Crystals }\end{array}$ & An & Ab & Or & $\begin{array}{c}\text { An } \\
\text { Std. }\end{array}$ & $\begin{array}{c}\text { Al } \\
\text { Std. }\end{array}$ & $\begin{array}{c}\text { Or } \\
\text { Std. }\end{array}$ & Latitude & Longitude \\
\hline MS-15-7b & 7 & 19.2 & 76.1 & 4.8 & 0.7 & 0.6 & 0.3 & 43.7469 & -118.7997 \\
MS-15-12 & 1 & 21.3 & 73.9 & 4.8 & N/A & N/A & N/A & 45.3672 & -118.1268 \\
MS-10-20 & 2 & 22.1 & 73.1 & 4.7 & 0.8 & 0.8 & 0.0 & 44.3378 & -117.9897 \\
MS-15-21a & 7 & 20.9 & 74.9 & 4.3 & 1.3 & 1.2 & 0.1 & 44.0092 & -118.8238 \\
MS-15-28 & 10 & 21.2 & 74.0 & 4.8 & 2.0 & 1.5 & 0.6 & 43.8754 & -119.0177 \\
MS-15-28.1b & 1 & 21.4 & 72.8 & 5.8 & 2.2 & 1.3 & 0.9 & 43.9349 & -119.0681 \\
MS-15-12.4 & 3 & 20.0 & 75.0 & 5.0 & 0.5 & 0.6 & 0.1 & 45.5850 & -117.8198 \\
MS-12-12.3 & 5 & 19.4 & 75.4 & 5.2 & 0.9 & 0.8 & 0.3 & 45.7367 & -117.8676 \\
MS-15-18.2 & 6 & 19.7 & 75.2 & 5.1 & 1.2 & 1.0 & 0.3 & 43.7033 & -118.8033 \\
MS-15-12.5 & 3 & 19.0 & 75.7 & 5.2 & 1.7 & 1.5 & 0.3 & 45.5588 & -117.9522 \\
\hline
\end{tabular}


Table B2. Unit 2 EMP major element individual feldspar crystal geochemical data.

\begin{tabular}{|c|c|c|c|c|c|c|c|c|c|}
\hline $\begin{array}{l}\text { Sample and } \\
\text { crystal } \\
\text { number** }\end{array}$ & $\mathrm{SiO}_{2}$ & $\mathrm{Al}_{2} \mathrm{O}_{3}$ & FeO* & MgO & $\mathrm{CaO}$ & $\mathrm{Na} 2 \mathrm{O}$ & $\mathbf{K}_{2} \mathbf{O}$ & BaO & Total \\
\hline \multicolumn{10}{|l|}{ MS-15-7b ${ }^{1}$} \\
\hline xl_1a & 61.21 & 24.04 & 0.24 & 0.000 & 4.17 & 9.27 & 0.87 & 0.21 & 100.0 \\
\hline $\mathrm{xl} \_1 \mathrm{~b}$ & 61.02 & 24.32 & 0.29 & 0.000 & 4.20 & 9.14 & 0.81 & 0.22 & 100.0 \\
\hline $\mathrm{xl} \_2 \mathrm{a}$ & 61.45 & 24.31 & 0.24 & 0.001 & 4.03 & 8.98 & 0.78 & 0.21 & 100.0 \\
\hline $\mathrm{xl} \_2 \mathrm{~b}$ & 61.64 & 24.08 & 0.19 & 0.000 & 4.14 & 8.87 & 0.88 & 0.20 & 100.0 \\
\hline $\mathrm{xl} \_3 \mathrm{a}$ & 61.27 & 24.13 & 0.22 & 0.006 & 4.17 & 9.11 & 0.89 & 0.21 & 100.0 \\
\hline$x l \_3 b$ & 60.74 & 24.44 & 0.29 & 0.000 & 4.40 & 9.14 & 0.79 & 0.21 & 100.0 \\
\hline $\mathrm{xl} \_4 \mathrm{a}$ & 61.19 & 24.26 & 0.26 & 0.000 & 4.01 & 9.15 & 0.91 & 0.22 & 100.0 \\
\hline$x l \_4 b$ & 61.01 & 24.22 & 0.30 & 0.000 & 4.10 & 9.23 & 0.90 & 0.25 & 100.0 \\
\hline xl_5a & 61.22 & 24.20 & 0.28 & 0.000 & 3.79 & 9.30 & 0.94 & 0.28 & 100.0 \\
\hline $\mathrm{xl} \_5 \mathrm{~b}$ & 61.30 & 24.24 & 0.18 & 0.005 & 4.17 & 8.96 & 0.91 & 0.23 & 100.0 \\
\hline xl_6a & 61.20 & 24.33 & 0.29 & 0.000 & 4.15 & 8.93 & 0.86 & 0.24 & 100.0 \\
\hline xl_6b & 61.34 & 24.17 & 0.26 & 0.000 & 4.29 & 8.83 & 0.86 & 0.24 & 100.0 \\
\hline $\mathrm{xl} \_7 \mathrm{a}$ & 61.29 & 24.21 & 0.25 & 0.000 & 4.05 & 9.09 & 0.87 & 0.25 & 100.0 \\
\hline $\mathrm{xl} \_7 \mathrm{~b}$ & 61.11 & 24.26 & 0.27 & 0.000 & 4.11 & 9.22 & 0.83 & 0.21 & 100.0 \\
\hline \multicolumn{10}{|l|}{ MS-15-12 } \\
\hline xl_1 & 62.93 & 23.46 & 0.25 & 0.000 & 4.25 & 8.19 & 0.80 & N/A & 99.9 \\
\hline \multicolumn{10}{|l|}{ MS-10-20 } \\
\hline $\mathrm{xl} \_2 \mathrm{a}$ & 64.51 & 23.86 & 0.20 & 0.001 & 4.26 & 8.09 & 0.79 & 0.18 & 101.9 \\
\hline xl_2b & 63.32 & 23.42 & 0.26 & 0.001 & 4.56 & 8.10 & 0.80 & 0.23 & 100.7 \\
\hline \multicolumn{10}{|l|}{ MS-15-21a } \\
\hline xl_1 & 62.96 & 22.68 & 0.23 & 0.003 & 4.42 & 8.10 & 0.79 & $\mathrm{~N} / \mathrm{A}$ & 99.2 \\
\hline xl_2 & 63.92 & 22.97 & 0.29 & 0.000 & 3.68 & 8.42 & 0.84 & N/A & 100.1 \\
\hline xl_3 & 64.05 & 22.69 & 0.20 & 0.000 & 3.83 & 8.37 & 0.85 & N/A & 100.0 \\
\hline xl_4 & 62.70 & 22.84 & 0.25 & 0.000 & 4.24 & 8.03 & 0.79 & N/A & 98.9 \\
\hline xl_5 & 63.58 & 22.86 & 0.21 & 0.002 & 4.23 & 7.93 & 0.81 & N/A & 99.6 \\
\hline xl_6 & 62.84 & 22.79 & 0.23 & 0.000 & 4.14 & 8.14 & 0.79 & N/A & 98.9 \\
\hline xl_7 & 63.79 & 22.55 & 0.30 & 0.000 & 4.12 & 8.10 & 0.78 & $\mathrm{~N} / \mathrm{A}$ & 99.7 \\
\hline \multicolumn{10}{|l|}{ MS-15-28 } \\
\hline $\mathrm{xl} \_1 \mathrm{a}$ & 65.12 & 22.63 & 0.24 & 0.000 & 3.82 & 8.37 & 0.92 & 0.24 & 101.3 \\
\hline xl_1b & 64.34 & 22.93 & 0.26 & 0.000 & 4.12 & 8.18 & 0.83 & 0.23 & 100.9 \\
\hline $\mathrm{xl} \_2 \mathrm{a}$ & 65.41 & 22.90 & 0.20 & 0.002 & 3.95 & 8.32 & 0.87 & 0.23 & 101.9 \\
\hline $\mathrm{xl} \_2 \mathrm{~b}$ & 64.72 & 22.91 & 0.22 & 0.000 & 3.98 & 8.06 & 0.88 & 0.28 & 101.1 \\
\hline $\mathrm{xl} 3 \mathrm{a}$ & 63.49 & 23.79 & 0.29 & 0.002 & 4.84 & 7.87 & 0.61 & 0.21 & 101.1 \\
\hline $\mathrm{xl} \_3 \mathrm{~b}$ & 63.50 & 23.53 & 0.26 & 0.000 & 4.84 & 7.58 & 0.64 & 0.23 & 100.6 \\
\hline xl_4a & 65.49 & 23.40 & 0.29 & 0.000 & 4.18 & 8.23 & 0.86 & 0.24 & 102.7 \\
\hline$x l \_4 b$ & 65.11 & 23.56 & 0.28 & 0.004 & 4.16 & 8.35 & 0.82 & 0.22 & 102.5 \\
\hline xl_5a & 64.38 & 22.74 & 0.20 & 0.000 & 3.88 & 8.12 & 0.78 & 0.29 & 100.4 \\
\hline
\end{tabular}


Table B2 continued.

\begin{tabular}{|c|c|c|c|c|c|c|c|c|c|}
\hline $\begin{array}{l}\text { Sample and } \\
\text { crystal } \\
\text { number** }\end{array}$ & $\mathrm{SiO}_{2}$ & $\mathrm{Al}_{2} \mathrm{O}_{3}$ & $\mathrm{FeO}^{*}$ & MgO & $\mathrm{CaO}$ & $\mathrm{Na}_{2} \mathrm{O}$ & $\mathrm{K}_{2} \mathrm{O}$ & $\mathrm{BaO}$ & Total \\
\hline \multicolumn{10}{|l|}{ MS-15-28 cont. } \\
\hline $\mathrm{xl} \_5 \mathrm{~b}$ & 64.29 & 22.86 & 0.29 & 0.000 & 4.01 & 8.08 & 0.72 & 0.25 & 100.5 \\
\hline xl_6a & 64.80 & 23.25 & 0.21 & 0.000 & 4.27 & 8.34 & 0.78 & 0.19 & 101.8 \\
\hline$x l \_6 b$ & 64.53 & 22.74 & 0.21 & 0.000 & 4.08 & 7.91 & 0.83 & 0.24 & 100.5 \\
\hline $\mathrm{xl} \_7 \mathrm{a}$ & 64.38 & 23.65 & 0.24 & 0.000 & 4.41 & 8.27 & 0.69 & 0.23 & 101.9 \\
\hline $\mathrm{xl} \_7 \mathrm{~b}$ & 62.80 & 24.38 & 0.32 & 0.000 & 5.34 & 7.64 & 0.57 & 0.16 & 101.2 \\
\hline $\mathrm{xl} \_8 \mathrm{a}$ & 63.73 & 23.73 & 0.17 & 0.000 & 4.20 & 8.27 & 0.79 & 0.21 & 101.1 \\
\hline $\mathrm{xl} \_8 \mathrm{~b}$ & 63.89 & 23.02 & 0.23 & 0.001 & 4.09 & 8.27 & 0.89 & 0.21 & 100.6 \\
\hline xl_9a & 63.68 & 23.08 & 0.27 & 0.000 & 4.13 & 8.36 & 0.82 & 0.23 & 100.6 \\
\hline xl_9b & 63.99 & 23.31 & 0.25 & 0.000 & 4.00 & 8.49 & 0.86 & 0.22 & 101.1 \\
\hline $\mathrm{xl} \_10 \mathrm{a}$ & 64.48 & 23.07 & 0.25 & 0.001 & 3.95 & 8.09 & 0.85 & 0.26 & 101.0 \\
\hline xl_10b & 64.45 & 22.87 & 0.17 & 0.000 & 3.85 & 8.11 & 0.86 & 0.30 & 100.6 \\
\hline \multicolumn{10}{|l|}{ MS-15-28.1b } \\
\hline xl_5a & 62.61 & 23.46 & 0.30 & 0.003 & 4.71 & 8.19 & 0.89 & 0.24 & 100.4 \\
\hline xl_5b & 64.11 & 22.67 & 0.33 & 0.006 & 3.85 & 7.93 & 1.04 & 0.26 & 100.2 \\
\hline \multicolumn{10}{|l|}{ MS-15-12.4 } \\
\hline xl_7a & 64.09 & 22.99 & 0.22 & 0.000 & 4.11 & 8.46 & 0.89 & 0.27 & 101.0 \\
\hline $\mathrm{xl} \_7 \mathrm{~b}$ & 64.97 & 23.13 & 0.17 & 0.008 & 4.08 & 8.16 & 0.85 & 0.23 & 101.6 \\
\hline xl_9a & 64.49 & 22.93 & 0.24 & 0.000 & 3.95 & 8.46 & 0.83 & 0.22 & 101.1 \\
\hline xl_9b & 63.97 & 22.94 & 0.27 & 0.004 & 3.97 & 8.49 & 0.83 & 0.24 & 100.7 \\
\hline \multicolumn{10}{|l|}{ MS-15-12.3 } \\
\hline $\mathrm{xl} \_2 \mathrm{a}$ & 63.97 & 22.50 & 0.22 & 0.000 & 3.49 & 8.41 & 0.99 & 0.31 & 99.9 \\
\hline $\mathrm{xl} \_2 \mathrm{~b}$ & 65.22 & 22.85 & 0.19 & 0.005 & 3.66 & 8.64 & 0.86 & 0.26 & 101.7 \\
\hline xl_3a & 63.72 & 23.02 & 0.22 & 0.000 & 3.94 & 8.10 & 0.82 & 0.24 & 100.1 \\
\hline$x l \_3 b$ & 63.76 & 22.48 & 0.29 & 0.000 & 3.92 & 8.30 & 0.86 & 0.22 & 99.8 \\
\hline$x 1 \_4 a$ & 63.87 & 22.67 & 0.23 & 0.000 & 3.91 & 8.34 & 0.90 & 0.24 & 100.2 \\
\hline $\mathrm{xl} \_4 \mathrm{~b}$ & 63.61 & 23.04 & 0.28 & 0.000 & 4.10 & 8.31 & 0.81 & 0.21 & 100.4 \\
\hline xl_5a & 64.10 & 23.29 & 0.23 & 0.000 & 4.19 & 8.42 & 0.87 & 0.23 & 101.3 \\
\hline $\mathrm{xl} \_5 \mathrm{~b}$ & 63.94 & 22.86 & 0.21 & 0.000 & 3.93 & 8.24 & 0.84 & 0.22 & 100.3 \\
\hline xl_6a & 64.86 & 22.52 & 0.16 & 0.000 & 3.58 & 8.50 & 0.92 & 0.36 & 100.9 \\
\hline xl_6b & 63.32 & 22.58 & 0.21 & 0.000 & 3.87 & 8.05 & 0.86 & 0.24 & 99.1 \\
\hline $\mathrm{xl} \_8 \mathrm{a}$ & 64.08 & 22.70 & 0.18 & 0.000 & 3.90 & 8.37 & 0.86 & 0.22 & 100.3 \\
\hline $\mathrm{xl} \_8 \mathrm{~b}$ & 64.21 & 22.71 & 0.23 & 0.000 & 3.99 & 8.31 & 0.86 & 0.23 & 100.5 \\
\hline xl_9a & 63.92 & 22.81 & 0.26 & 0.000 & 4.05 & 8.38 & 0.89 & 0.27 & 100.6 \\
\hline xl_9b & 63.90 & 22.65 & 0.26 & 0.004 & 3.73 & 8.51 & 0.87 & 0.22 & 100.1 \\
\hline xl_10a & 63.88 & 22.61 & 0.25 & 0.006 & 3.83 & 8.31 & 0.87 & 0.23 & 100.0 \\
\hline $\mathrm{xl} \_10 \mathrm{~b}$ & 64.45 & 22.81 & 0.25 & 0.000 & 3.95 & 8.57 & 0.89 & 0.23 & 101.1 \\
\hline
\end{tabular}


Table B2 continued.

\begin{tabular}{|c|c|c|c|c|c|c|c|c|c|}
\hline $\begin{array}{l}\text { Sample and } \\
\text { crystal } \\
\text { number** }\end{array}$ & $\mathrm{SiO}_{2}$ & $\mathrm{Al}_{2} \mathrm{O}_{3}$ & $\mathrm{FeO}^{*}$ & MgO & $\mathrm{CaO}$ & $\mathrm{Na}_{2} \mathrm{O}$ & $\mathrm{K}_{2} \mathrm{O}$ & $\mathrm{BaO}$ & Total \\
\hline \multicolumn{10}{|l|}{ MS-15-8.2 } \\
\hline xl_1a & 62.85 & 22.61 & 0.27 & 0.000 & 3.64 & 8.44 & 0.91 & 0.32 & 99.0 \\
\hline xl_1b & 62.24 & 22.62 & 0.25 & 0.000 & 3.56 & 8.32 & 0.90 & 0.24 & 98.1 \\
\hline $\mathrm{xl} \_2 \mathrm{a}$ & 61.96 & 22.75 & 0.23 & 0.006 & 4.17 & 8.15 & 0.79 & 0.22 & 98.3 \\
\hline$x l \_2 b$ & 62.67 & 23.22 & 0.24 & 0.000 & 3.99 & 8.28 & 0.87 & 0.24 & 99.5 \\
\hline xl_4a & 62.34 & 22.85 & 0.24 & 0.000 & 3.95 & 8.19 & 0.80 & 0.17 & 98.5 \\
\hline $\mathrm{xl} \_4 \mathrm{~b}$ & 62.47 & 22.19 & 0.24 & 0.000 & 3.63 & 8.18 & 0.89 & 0.25 & 97.9 \\
\hline xl_5a & 62.13 & 22.90 & 0.24 & 0.001 & 3.85 & 8.34 & 0.85 & 0.22 & 98.5 \\
\hline $\mathrm{xl} \_5 \mathrm{~b}$ & 62.54 & 22.73 & 0.21 & 0.000 & 4.12 & 7.96 & 0.80 & 0.18 & 98.5 \\
\hline xl_6a & 64.46 & 22.39 & 0.25 & 0.000 & 3.67 & 8.52 & 0.87 & 0.25 & 100.4 \\
\hline xl_6b & 62.05 & 22.44 & 0.21 & 0.000 & 3.90 & 8.12 & 0.80 & 0.21 & 97.7 \\
\hline xl_7a & 61.80 & 22.24 & 0.17 & 0.000 & 3.90 & 8.02 & 0.86 & 0.22 & 97.2 \\
\hline $\mathrm{xl} \_7 \mathrm{~b}$ & 60.43 & 22.49 & 0.26 & 0.000 & 4.07 & 7.80 & 0.77 & 0.21 & 96.0 \\
\hline \multicolumn{10}{|l|}{ MS-15-12.5 } \\
\hline xl_4Ka & 64.54 & 22.96 & 0.24 & 0.000 & 3.81 & 8.71 & 0.92 & 0.22 & 101.4 \\
\hline $\mathrm{xl} \_4 \mathrm{~Kb}$ & 63.73 & 22.90 & 0.23 & 0.000 & 3.96 & 8.73 & 0.86 & 0.22 & 100.6 \\
\hline $\mathrm{xl} \_4 \mathrm{Kc}$ & 64.14 & 22.69 & 0.23 & 0.000 & 3.51 & 8.84 & 0.91 & 0.31 & 100.6 \\
\hline xl_8Ja & 63.71 & 23.25 & 0.20 & 0.000 & 4.02 & 8.46 & 0.84 & 0.28 & 100.8 \\
\hline $\mathrm{xl} \_8 \mathrm{Jb}$ & 63.12 & 22.46 & 0.24 & 0.000 & 4.18 & 8.12 & 0.85 & 0.27 & 99.2 \\
\hline xl_9Ja & 63.64 & 22.67 & 0.21 & 0.000 & 4.08 & 8.26 & 0.90 & 0.24 & 100.0 \\
\hline xl_9Jb & 64.83 & 22.30 & 0.24 & 0.000 & 3.30 & 8.65 & 0.96 & 0.33 & 100.6 \\
\hline
\end{tabular}


Table B3. Unit 2 individual feldspar crystal end member compositions.

\begin{tabular}{|c|c|c|c|}
\hline Sample and crystal number** & $\mathbf{A n}^{2}$ & $\mathbf{A b ^ { 3 }}$ & $\mathrm{Or}^{4}$ \\
\hline \multicolumn{4}{|l|}{ MS-15-7b ${ }^{1}$} \\
\hline $\mathrm{xl} \_1 \mathrm{a}$ & 19.04 & 76.25 & 4.72 \\
\hline $\mathrm{xl} \_1 \mathrm{~b}$ & 19.41 & 76.15 & 4.45 \\
\hline $\mathrm{xl} \_2 \mathrm{a}$ & 19.08 & 76.52 & 4.40 \\
\hline $\mathrm{xl} \_2 \mathrm{~b}$ & 19.56 & 75.51 & 4.94 \\
\hline $\mathrm{xl} \_3 \mathrm{a}$ & 19.25 & 75.87 & 4.89 \\
\hline $\mathrm{xl} \_3 \mathrm{~b}$ & 20.17 & 75.52 & 4.32 \\
\hline $\mathrm{xl} \_4 \mathrm{a}$ & 18.58 & 76.42 & 5.01 \\
\hline $\mathrm{xl} \_4 \mathrm{~b}$ & 18.80 & 76.30 & 4.90 \\
\hline $\mathrm{xl} \_5 \mathrm{a}$ & 17.48 & 77.35 & 5.17 \\
\hline $\mathrm{xl} \_5 \mathrm{~b}$ & 19.50 & 75.46 & 5.05 \\
\hline xl_6a & 19.51 & 75.66 & 4.83 \\
\hline xl_6b & 20.21 & 74.95 & 4.85 \\
\hline $\mathrm{xl} \_7 \mathrm{a}$ & 18.86 & 76.32 & 4.82 \\
\hline xl_7b & 18.94 & 76.52 & 4.55 \\
\hline \multicolumn{4}{|l|}{ MS-15-12 } \\
\hline xl_1 & 21.29 & 73.91 & 4.80 \\
\hline \multicolumn{4}{|l|}{ MS-10-20 } \\
\hline $\mathrm{xl} \_2 \mathrm{a}$ & 21.56 & 73.71 & 4.73 \\
\hline xl_2b & 22.66 & 72.58 & 4.76 \\
\hline \multicolumn{4}{|l|}{ MS-15-21a } \\
\hline xl_1 & 22.15 & 73.15 & 4.70 \\
\hline xl_2 & 18.56 & 76.42 & 5.02 \\
\hline xl_3 & 19.23 & 75.69 & 5.08 \\
\hline xl_4 & 21.57 & 73.64 & 4.79 \\
\hline xl_5 & 21.69 & 73.35 & 4.97 \\
\hline xl_6 & 20.96 & 74.26 & 4.78 \\
\hline xl_7 & 20.96 & 74.29 & 4.76 \\
\hline \multicolumn{4}{|l|}{ MS-15-28 } \\
\hline xl_1a & 19.09 & 75.42 & 5.49 \\
\hline xl_1b & 20.77 & 74.27 & 4.97 \\
\hline $\mathrm{xl} \_2 \mathrm{a}$ & 19.79 & 75.03 & 5.19 \\
\hline $\mathrm{xl} \_2 \mathrm{~b}$ & 20.36 & 74.28 & 5.37 \\
\hline xl_3a & 24.51 & 71.82 & 3.68 \\
\hline $\mathrm{xl} \_3 \mathrm{~b}$ & 25.10 & 70.93 & 3.97 \\
\hline xl_4a & 20.85 & 74.05 & 5.10 \\
\hline$x 1 \_4 b$ & 20.60 & 74.57 & 4.83 \\
\hline xl_5a & 19.96 & 75.29 & 4.76 \\
\hline xl_5b & 20.61 & 74.94 & 4.45 \\
\hline
\end{tabular}


Table B3 continued.

\begin{tabular}{|c|c|c|c|}
\hline Sample and crystal number** & $\mathbf{A n}^{2}$ & $\mathbf{A b}^{3}$ & $\mathrm{Or}^{4}$ \\
\hline \multicolumn{4}{|l|}{ MS-15-28 cont. } \\
\hline xl_6a & 21.10 & 74.32 & 4.58 \\
\hline $\mathrm{xl} \_6 \mathrm{~b}$ & 21.13 & 73.77 & 5.11 \\
\hline $\mathrm{xl} \_7 \mathrm{a}$ & 21.89 & 74.01 & 4.10 \\
\hline $\mathrm{xl} \_7 \mathrm{~b}$ & 26.97 & 69.57 & 3.46 \\
\hline $\mathrm{xl} \_8 \mathrm{a}$ & 20.96 & 74.34 & 4.70 \\
\hline $\mathrm{xl} \_8 \mathrm{~b}$ & 20.41 & 74.32 & 5.27 \\
\hline xl_9a & 20.45 & 74.72 & 4.83 \\
\hline xl_9b & 19.65 & 75.28 & 5.07 \\
\hline xl_10a & 20.21 & 74.58 & 5.21 \\
\hline $\mathrm{xl} \_10 \mathrm{~b}$ & 19.75 & 74.97 & 5.29 \\
\hline \multicolumn{4}{|l|}{ MS-15-28.1b } \\
\hline xl_5a & 22.92 & 71.91 & 5.18 \\
\hline $\mathrm{xl} \_5 \mathrm{~b}$ & 19.86 & 73.73 & 6.42 \\
\hline \multicolumn{4}{|l|}{ MS-15-12.4 } \\
\hline $\mathrm{xl} \_7 \mathrm{a}$ & 20.13 & 74.71 & 5.17 \\
\hline $\mathrm{xl} \_7 \mathrm{~b}$ & 20.63 & 74.26 & 5.12 \\
\hline xl_9a & 19.55 & 75.54 & 4.92 \\
\hline xl_9b & 19.59 & 75.50 & 4.91 \\
\hline \multicolumn{4}{|l|}{ MS-15-12.3 } \\
\hline xl_2a & 17.60 & 76.43 & 5.97 \\
\hline $\mathrm{xl} \_2 \mathrm{~b}$ & 18.06 & 76.87 & 5.07 \\
\hline xl_3a & 20.17 & 74.80 & 5.03 \\
\hline$x l \_3 b$ & 19.68 & 75.20 & 5.12 \\
\hline $\mathrm{xl} \_4 \mathrm{a}$ & 19.54 & 75.12 & 5.35 \\
\hline$x l \_4 b$ & 20.45 & 74.72 & 4.83 \\
\hline xl_5a & 20.53 & 74.42 & 5.06 \\
\hline $\mathrm{xl} \_5 \mathrm{~b}$ & 19.87 & 75.05 & 5.08 \\
\hline xl_6a & 17.92 & 76.59 & 5.49 \\
\hline xl_6b & 19.94 & 74.80 & 5.27 \\
\hline xl_8a & 19.48 & 75.40 & 5.12 \\
\hline $\mathrm{xl} \_8 \mathrm{~b}$ & 19.95 & 74.95 & 5.11 \\
\hline xl_9a & 20.04 & 74.69 & 5.27 \\
\hline xl_9b & 18.54 & 76.31 & 5.15 \\
\hline xl_10a & 19.30 & 75.48 & 5.23 \\
\hline xl_10b & 19.29 & 75.51 & 5.20 \\
\hline \multicolumn{4}{|l|}{ MS-15-18.2 } \\
\hline $\mathrm{xl} \_1 \mathrm{a}$ & 18.24 & 76.30 & 5.47 \\
\hline $\mathrm{xl} \_1 \mathrm{~b}$ & 18.15 & 76.41 & 5.44 \\
\hline $\mathrm{xl} \_2 \mathrm{a}$ & 21.05 & 74.17 & 4.78 \\
\hline
\end{tabular}


Table B3 continued.

\begin{tabular}{|c|c|c|c|}
\hline Sample and crystal number** & $\mathbf{A n ^ { 2 }}$ & $\mathbf{A b ^ { 3 }}$ & $\mathrm{Or}^{4}$ \\
\hline \multicolumn{4}{|l|}{ MS-15-18.2 cont. } \\
\hline $\mathrm{xl} \_2 \mathrm{~b}$ & 19.98 & 74.82 & 5.21 \\
\hline $\mathrm{xl} \_4 \mathrm{a}$ & 20.09 & 75.04 & 4.88 \\
\hline $\mathrm{xl} \_4 \mathrm{~b}$ & 18.67 & 75.86 & 5.48 \\
\hline $\mathrm{xl} \_5 \mathrm{a}$ & 19.32 & 75.58 & 5.10 \\
\hline $\mathrm{xl} \_5 \mathrm{~b}$ & 21.19 & 73.88 & 4.93 \\
\hline xl_6a & 18.29 & 76.52 & 5.20 \\
\hline $\mathrm{xl} \_6 \mathrm{~b}$ & 20.03 & 75.07 & 4.90 \\
\hline xl_7a & 20.13 & 74.60 & 5.28 \\
\hline $\mathrm{xl} \_7 \mathrm{~b}$ & 21.34 & 73.81 & 4.85 \\
\hline \multicolumn{4}{|l|}{ MS-15-12.5 } \\
\hline xl_4Ka & 18.50 & 76.19 & 5.31 \\
\hline $\mathrm{xl} \_4 \mathrm{~Kb}$ & 19.09 & 75.98 & 4.93 \\
\hline $\mathrm{xl} \_4 \mathrm{Kc}$ & 17.11 & 77.61 & 5.28 \\
\hline xl_8Ja & 19.81 & 75.24 & 4.95 \\
\hline $\mathrm{xl} \_8 \mathrm{Jb}$ & 21.07 & 73.85 & 5.08 \\
\hline xl_9Ja & 20.37 & 74.27 & 5.36 \\
\hline xl_9Jb & 16.46 & 77.83 & 5.72 \\
\hline $\begin{array}{l}\text { ** Letters following crystal nun } \\
\text { 1. Normalized values } \\
\text { 2. } \mathrm{An}=\left(\mathrm{CaO}^{*} 0.714 / 40\right) /(\mathrm{CaO} * \\
\text { 3. } \mathrm{Ab}=\left(\mathrm{Na}_{2} \mathrm{O} * 0.74 / 23\right) /(\mathrm{CaO} * \\
\text { 4. } \mathrm{Or}=\left(\mathrm{K}_{2} \mathrm{O} * 0.83 / 39\right) /\left(\mathrm{CaO}^{*} 0 .\right.\end{array}$ & $\begin{array}{l}\text { per crys } \\
9)^{*} 100 \\
9{ }^{*} 100 \\
* 100\end{array}$ & & \\
\hline
\end{tabular}




\section{APPENDIX C: UNIT 3 CHEMICAL DATA, STANDARD DEVIATIONS AND GPS COORDINATES}

Table C1. Unit 3 average feldspar end member component composition and GPS coordinates by sample.

\begin{tabular}{lccccccccc}
\hline \hline Sample & $\begin{array}{c}\text { Number of } \\
\text { Crystals }\end{array}$ & An & Ab & Or & $\begin{array}{c}\text { An } \\
\text { Std. }\end{array}$ & $\begin{array}{c}\text { Al } \\
\text { Std. }\end{array}$ & $\begin{array}{c}\text { Or } \\
\text { Std. }\end{array}$ & Latitude & Longitude \\
\hline MS-12-33.1 & 12 & 4.2 & 78.7 & 17.1 & 1.1 & 0.7 & 1.3 & 44.0402 & -117.5092 \\
EBC-12-074 & 10 & 4.4 & 79.1 & 16.6 & 0.9 & 0.8 & 1.0 & 44.6639 & -118.4302 \\
MS-14-12 & 10 & 3.9 & 79.1 & 17.0 & 0.5 & 0.4 & 0.7 & 44.5066 & -117.8920 \\
MS-13-08 & 10 & 4.0 & 73.3 & 22.7 & 1.0 & 0.9 & 1.7 & 43.4379 & -118.5243 \\
MS-10-26 & 1 & 3.5 & 72.0 & 24.4 & N/A & N/A & N/A & 43.9226 & -118.9579 \\
MS-10-25 & 7 & 4.9 & 75.1 & 20.1 & 0.3 & 1.5 & 1.6 & 43.9025 & -119.0301 \\
PFC-1513 & 10 & 4.4 & 78.4 & 17.2 & 0.5 & 0.4 & 0.6 & 43.9985 & -118.8488 \\
\hline
\end{tabular}


Table C2. Unit 3 EMP major element feldspar chemical composition by sample.

\begin{tabular}{|c|c|c|c|c|c|c|c|c|c|}
\hline $\begin{array}{l}\text { Sample and } \\
\text { crystal } \\
\text { number** }\end{array}$ & $\mathrm{SiO}_{2}$ & $\mathrm{Al}_{2} \mathrm{O}_{3}$ & $\mathrm{FeO}$ & MgO & $\mathrm{CaO}$ & $\mathrm{Na} 2 \mathrm{O}$ & $\mathrm{K}_{2} \mathrm{O}$ & $\mathrm{BaO}$ & Total \\
\hline \multicolumn{10}{|l|}{ MS-12-33.1-C } \\
\hline xl_2 & 67.65 & 21.11 & 0.28 & 0.000 & 0.91 & 8.82 & 2.69 & 0.98 & 102.5 \\
\hline xl_6 & 67.01 & 20.57 & 0.21 & 0.000 & 0.94 & 8.69 & 2.84 & 0.98 & 101.2 \\
\hline \multicolumn{10}{|l|}{ MS-12-33.1-H } \\
\hline xl_1a & 68.49 & 20.12 & 0.19 & 0.000 & 0.71 & 8.56 & 2.89 & 0.88 & 101.8 \\
\hline xl_1b & 68.47 & 20.11 & 0.19 & 0.009 & 0.73 & 8.62 & 3.08 & 0.93 & 102.1 \\
\hline $\mathrm{xl} \_2 \mathrm{a}$ & 69.75 & 19.57 & 0.29 & 0.000 & 0.37 & 8.71 & 3.26 & 0.51 & 102.5 \\
\hline xl_2b & 68.58 & 20.10 & 0.27 & 0.000 & 0.65 & 8.73 & 2.69 & 0.49 & 101.5 \\
\hline xl_3a & 67.28 & 20.85 & 0.19 & 0.000 & 1.52 & 8.68 & 2.23 & 1.01 & 101.8 \\
\hline xl_3b & 68.65 & 20.55 & 0.24 & 0.000 & 0.87 & 8.75 & 2.69 & 0.92 & 102.7 \\
\hline xl_4a & 68.21 & 20.41 & 0.23 & 0.001 & 0.70 & 8.60 & 2.96 & 1.06 & 102.2 \\
\hline xl_4b & 67.99 & 20.19 & 0.20 & 0.000 & 0.78 & 8.35 & 3.03 & 1.04 & 101.6 \\
\hline xl_5a & 66.50 & 20.20 & 0.21 & 0.000 & 1.04 & 8.28 & 2.50 & 1.02 & 99.8 \\
\hline xl_5b & 67.93 & 20.27 & 0.26 & 0.000 & 0.91 & 8.58 & 2.67 & 0.99 & 101.6 \\
\hline xl_6a & 69.02 & 20.32 & 0.27 & 0.005 & 0.75 & 8.75 & 2.89 & 0.98 & 103.0 \\
\hline xl_6b & 68.35 & 20.16 & 0.24 & 0.000 & 0.65 & 8.54 & 3.03 & 1.01 & 102.0 \\
\hline xl_8a & 68.46 & 20.42 & 0.28 & 0.000 & 0.90 & 8.46 & 2.90 & 1.08 & 102.5 \\
\hline xl_8b & 66.87 & 20.46 & 0.23 & 0.007 & 0.92 & 8.39 & 2.80 & 0.96 & 100.6 \\
\hline xl_9a & 67.11 & 20.28 & 0.23 & 0.000 & 0.86 & 8.48 & 2.69 & 1.02 & 100.7 \\
\hline xl_9b & 66.84 & 20.16 & 0.20 & 0.000 & 0.87 & 8.57 & 2.84 & 0.95 & 100.4 \\
\hline xl_10a & 67.91 & 20.29 & 0.28 & 0.000 & 0.73 & 8.51 & 3.08 & 1.11 & 101.9 \\
\hline xl_10b & 68.58 & 20.16 & 0.24 & 0.001 & 0.67 & 8.83 & 3.06 & 1.05 & 102.6 \\
\hline \multicolumn{10}{|l|}{ EBC12-074 } \\
\hline xl_1a & 62.74 & 20.02 & 0.26 & 0.014 & 0.91 & 8.35 & 2.57 & 0.87 & 95.7 \\
\hline xl_1b & 61.95 & 19.70 & 0.26 & 0.000 & 0.91 & 8.09 & 2.61 & 0.94 & 94.4 \\
\hline $\mathrm{xl} \_2 \mathrm{a}$ & 61.46 & 19.97 & 0.23 & 0.000 & 0.93 & 8.49 & 2.61 & 0.90 & 94.6 \\
\hline $\mathrm{xl} \_2 \mathrm{~b}$ & 62.69 & 19.81 & 0.20 & 0.000 & 0.82 & 8.62 & 2.54 & 0.77 & 95.5 \\
\hline xl_3a & 61.39 & 19.95 & 0.21 & 0.005 & 0.92 & 8.20 & 2.67 & 0.95 & 94.3 \\
\hline$x l \_3 b$ & 61.87 & 20.07 & 0.22 & 0.000 & 0.74 & 8.30 & 2.74 & 0.74 & 94.7 \\
\hline xl_4a & 62.20 & 20.05 & 0.24 & 0.002 & 0.93 & 8.39 & 2.66 & 0.89 & 95.4 \\
\hline xl_4b & 61.05 & 19.60 & 0.19 & 0.000 & 0.89 & 8.64 & 2.73 & 0.95 & 94.1 \\
\hline xl_5a & 62.60 & 19.85 & 0.22 & 0.000 & 0.83 & 8.38 & 2.69 & 0.94 & 95.5 \\
\hline xl_5b & 62.63 & 19.89 & 0.24 & 0.000 & 0.91 & 8.57 & 2.71 & 0.88 & 95.8 \\
\hline xl_6a & 61.65 & 19.58 & 0.22 & 0.001 & 0.76 & 8.26 & 2.78 & 0.88 & 94.1 \\
\hline xl_6b & 61.93 & 20.11 & 0.18 & 0.000 & 0.82 & 8.25 & 2.73 & 0.91 & 94.9 \\
\hline xl_7a & 62.54 & 19.38 & 0.24 & 0.002 & 0.51 & 8.55 & 2.86 & 0.76 & 94.8 \\
\hline$x l \_7 b$ & 62.14 & 19.19 & 0.26 & 0.005 & 0.47 & 8.54 & 2.45 & 0.69 & 93.7 \\
\hline xl_8a & 63.06 & 20.11 & 0.18 & 0.000 & 1.09 & 8.36 & 2.43 & 1.18 & 96.4 \\
\hline Table $\mathrm{C} 2$ contin & & & & & & & & & \\
\hline
\end{tabular}




\begin{tabular}{|c|c|c|c|c|c|c|c|c|c|}
\hline $\begin{array}{l}\text { Sample and } \\
\text { crystal } \\
\text { number*** }\end{array}$ & $\mathrm{SiO}_{2}$ & $\mathrm{Al}_{2} \mathrm{O}_{3}$ & $\mathrm{FeO}^{*}$ & MgO & $\mathrm{CaO}$ & $\mathrm{Na}_{2} \mathrm{O}$ & $\mathbf{K}_{2} \mathbf{O}$ & $\mathrm{BaO}$ & Total \\
\hline \multicolumn{10}{|l|}{$\begin{array}{l}\text { EBC12-074 } \\
\text { cont. }\end{array}$} \\
\hline $\mathrm{xl} \_8 \mathrm{~b}$ & 61.96 & 19.86 & 0.20 & 0.000 & 1.09 & 8.18 & 2.28 & 1.11 & 94.7 \\
\hline xl_9a & 62.29 & 19.97 & 0.17 & 0.000 & 0.99 & 8.43 & 2.60 & 0.91 & 95.4 \\
\hline xl_9b & 62.47 & 19.90 & 0.26 & 0.005 & 0.93 & 8.59 & 2.64 & 0.92 & 95.7 \\
\hline xl_10a & 61.98 & 19.92 & 0.23 & 0.000 & 0.76 & 8.28 & 2.80 & 0.88 & 94.8 \\
\hline xl_10b & 61.78 & 19.58 & 0.26 & 0.000 & 0.52 & 8.24 & 3.02 & 1.11 & 94.5 \\
\hline \multicolumn{10}{|l|}{ MS-14-12 } \\
\hline xl_1a & 66.86 & 20.36 & 0.22 & 0.000 & 0.68 & 8.76 & 2.93 & 1.15 & 101.0 \\
\hline $\mathrm{xl} \_1 \mathrm{~b}$ & 66.18 & 20.30 & 0.23 & 0.001 & 0.65 & 8.70 & 3.04 & 1.17 & 100.3 \\
\hline $\mathrm{xl} \_2 \mathrm{a}$ & 66.66 & 20.59 & 0.14 & 0.001 & 0.84 & 9.03 & 2.91 & 0.99 & 101.2 \\
\hline $\mathrm{xl} \_2 \mathrm{~b}$ & 66.99 & 20.85 & 0.17 & 0.003 & 0.87 & 9.05 & 2.78 & 1.00 & 101.7 \\
\hline$x 1 \_3 a$ & 67.86 & 20.49 & 0.25 & 0.000 & 0.62 & 8.89 & 3.09 & 0.90 & 102.1 \\
\hline $\mathrm{xl} \_3 \mathrm{~b}$ & 66.52 & 20.23 & 0.22 & 0.000 & 0.74 & 8.67 & 2.89 & 1.02 & 100.3 \\
\hline xl_4a & 67.90 & 20.67 & 0.25 & 0.000 & 0.79 & 9.06 & 2.90 & 0.99 & 102.6 \\
\hline $\mathrm{xl} \_4 \mathrm{~b}$ & 67.38 & 20.51 & 0.26 & 0.000 & 0.81 & 8.81 & 2.95 & 0.91 & 101.6 \\
\hline xl_5a & 67.17 & 20.19 & 0.24 & 0.000 & 0.64 & 9.06 & 2.93 & 0.94 & 101.2 \\
\hline $\mathrm{xl} \_5 \mathrm{~b}$ & 67.08 & 20.04 & 0.26 & 0.004 & 0.63 & 8.80 & 3.03 & 0.87 & 100.7 \\
\hline xl_6a & 68.13 & 20.66 & 0.25 & 0.000 & 0.83 & 8.75 & 2.94 & 0.99 & 102.5 \\
\hline xl_6b & 68.13 & 20.83 & 0.21 & 0.000 & 0.70 & 9.05 & 2.97 & 0.99 & 102.9 \\
\hline xl_7a & 67.52 & 20.74 & 0.23 & 0.000 & 0.85 & 8.94 & 2.81 & 0.98 & 102.1 \\
\hline $\mathrm{xl} \_7 \mathrm{~b}$ & 67.59 & 20.68 & 0.19 & 0.000 & 0.78 & 8.73 & 2.89 & 0.96 & 101.8 \\
\hline xl_8a & 67.14 & 20.77 & 0.19 & 0.000 & 0.88 & 8.91 & 2.81 & 1.00 & 101.7 \\
\hline $\mathrm{xl} \_8 \mathrm{~b}$ & 67.52 & 20.52 & 0.16 & 0.000 & 0.92 & 8.90 & 2.79 & 1.00 & 101.8 \\
\hline xl_9a & 66.53 & 20.71 & 0.22 & 0.000 & 1.01 & 8.95 & 2.70 & 0.99 & 101.1 \\
\hline xl_9b & 66.00 & 20.61 & 0.17 & 0.006 & 0.89 & 8.96 & 2.79 & 0.96 & 100.4 \\
\hline $\mathrm{xl} \_10 \mathrm{a}$ & 67.92 & 20.76 & 0.22 & 0.000 & 0.90 & 9.26 & 2.70 & 0.93 & 102.7 \\
\hline xl_10b & 66.55 & 20.38 & 0.19 & 0.000 & 0.85 & 8.83 & 2.89 & 0.94 & 100.6 \\
\hline \multicolumn{10}{|l|}{ MS-13-08 } \\
\hline $\mathrm{xl} \_1 \mathrm{a}$ & 65.87 & 19.92 & 0.24 & 0.001 & 0.79 & 7.88 & 4.03 & 0.66 & 99.4 \\
\hline $\mathrm{xl} \_1 \mathrm{~b}$ & 66.48 & 19.85 & 0.29 & 0.001 & 0.70 & 8.13 & 4.00 & 0.66 & 100.1 \\
\hline $\mathrm{xl} \_2 \mathrm{a}$ & 66.28 & 19.74 & 0.34 & 0.000 & 0.66 & 8.06 & 3.95 & 0.45 & 99.5 \\
\hline$x l \_2 b$ & 66.69 & 19.88 & 0.32 & 0.000 & 0.65 & 8.33 & 4.08 & 0.45 & 100.4 \\
\hline $\mathrm{xl} \_2 \mathrm{c}$ & 66.01 & 19.61 & 0.27 & 0.004 & 0.65 & 8.17 & 3.90 & 0.50 & 99.1 \\
\hline xl_3a & 66.14 & 19.76 & 0.24 & 0.000 & 0.80 & 7.98 & 3.80 & 0.62 & 99.3 \\
\hline$x l \_3 b$ & 65.98 & 20.31 & 0.27 & 0.000 & 0.82 & 8.10 & 3.80 & 0.61 & 99.9 \\
\hline$x l \_3 c$ & 66.66 & 19.95 & 0.32 & 0.002 & 0.70 & 8.18 & 3.95 & 0.52 & 100.3 \\
\hline xl_4a & 65.67 & 20.26 & 0.23 & 0.000 & 1.25 & 8.10 & 3.20 & 0.76 & 99.5 \\
\hline$x l \_4 b$ & 65.98 & 20.42 & 0.31 & 0.001 & 1.21 & 8.40 & 3.16 & 0.71 & 100.2 \\
\hline
\end{tabular}


Table C2 continued.

\begin{tabular}{|c|c|c|c|c|c|c|c|c|c|}
\hline $\begin{array}{l}\text { Sample and } \\
\text { crystal } \\
\text { number** }\end{array}$ & $\mathrm{SiO}_{2}$ & $\mathrm{Al}_{2} \mathrm{O}_{3}$ & $\mathrm{FeO}^{*}$ & MgO & $\mathrm{CaO}$ & $\mathrm{Na}_{2} \mathrm{O}$ & $\mathrm{K}_{2} \mathrm{O}$ & $\mathrm{BaO}$ & Total \\
\hline \multicolumn{10}{|l|}{ MS-13-08 cont. } \\
\hline $\mathrm{xl} \_4 \mathrm{c}$ & 65.66 & 20.47 & 0.24 & 0.000 & 1.24 & 8.05 & 3.11 & 0.71 & 99.5 \\
\hline xl_5a & 67.14 & 19.93 & 0.23 & 0.000 & 0.76 & 8.23 & 4.05 & 0.57 & 100.9 \\
\hline $\mathrm{xl} \_5 \mathrm{~b}$ & 66.82 & 19.73 & 0.38 & 0.000 & 0.70 & 8.01 & 3.89 & 0.46 & 100.0 \\
\hline $\mathrm{xl} \_5 \mathrm{c}$ & 66.86 & 19.84 & 0.31 & 0.000 & 0.65 & 7.91 & 3.99 & 0.45 & 100.0 \\
\hline xl_6a & 66.87 & 19.91 & 0.28 & 0.000 & 0.59 & 8.01 & 4.08 & 0.45 & 100.2 \\
\hline $\mathrm{xl} \_6 \mathrm{~b}$ & 66.81 & 20.11 & 0.30 & 0.000 & 0.66 & 8.39 & 3.98 & 0.52 & 100.8 \\
\hline $\mathrm{xl} \_6 \mathrm{c}$ & 67.23 & 20.04 & 0.20 & 0.000 & 0.79 & 8.14 & 3.95 & 0.55 & 100.9 \\
\hline $\mathrm{xl} \_7 \mathrm{a}$ & 65.89 & 20.07 & 0.24 & 0.000 & 1.13 & 8.10 & 3.19 & 0.58 & 99.2 \\
\hline $\mathrm{xl} \_7 \mathrm{~b}$ & 66.50 & 20.05 & 0.25 & 0.000 & 0.95 & 8.20 & 3.46 & 0.50 & 99.9 \\
\hline $\mathrm{xl} \_7 \mathrm{c}$ & 67.14 & 20.36 & 0.29 & 0.000 & 0.93 & 8.20 & 3.54 & 0.63 & 101.1 \\
\hline $\mathrm{xl} \_8 \mathrm{a}$ & 66.60 & 19.76 & 0.28 & 0.000 & 0.67 & 8.26 & 3.89 & 0.45 & 99.9 \\
\hline $\mathrm{xl} \_8 \mathrm{~b}$ & 67.57 & 19.80 & 0.29 & 0.000 & 0.71 & 8.57 & 3.91 & 0.45 & 101.3 \\
\hline $\mathrm{xl} \_8 \mathrm{c}$ & 67.38 & 19.86 & 0.25 & 0.000 & 0.69 & 8.28 & 3.88 & 0.46 & 100.8 \\
\hline xl_9a & 66.94 & 20.18 & 0.25 & 0.000 & 0.85 & 7.90 & 3.82 & 0.68 & 100.6 \\
\hline xl_9b & 66.62 & 20.17 & 0.19 & 0.000 & 0.84 & 8.16 & 3.77 & 0.65 & 100.4 \\
\hline xl_9c & 66.04 & 20.07 & 0.26 & 0.000 & 0.87 & 7.95 & 3.84 & 0.57 & 99.6 \\
\hline xl_10a & 67.23 & 20.14 & 0.31 & 0.000 & 0.69 & 8.03 & 3.97 & 0.54 & 100.9 \\
\hline xl_10b & 67.02 & 20.16 & 0.32 & 0.000 & 0.69 & 7.94 & 4.04 & 0.55 & 100.7 \\
\hline xl_10c & 67.53 & 19.97 & 0.28 & 0.000 & 0.70 & 8.16 & 4.15 & 0.53 & 101.3 \\
\hline \multicolumn{10}{|l|}{ MS-10-26 } \\
\hline xl_2 & 65.88 & 20.07 & 0.24 & 0.002 & 0.69 & 7.78 & 3.99 & $\mathrm{~N} / \mathrm{A}$ & 98.7 \\
\hline \multicolumn{10}{|l|}{ MS-10-25 } \\
\hline xl_1 & 67.70 & 20.54 & 0.24 & 0.000 & 0.83 & 7.97 & 2.91 & N/A & 100.2 \\
\hline xl_2 & 68.51 & 20.36 & 0.17 & 0.000 & 0.95 & 8.57 & 2.84 & N/A & 101.4 \\
\hline xl_3 & 67.59 & 20.34 & 0.27 & 0.000 & 0.82 & 7.91 & 3.00 & N/A & 99.9 \\
\hline xl_4 & 68.68 & 20.38 & 0.21 & 0.000 & 0.91 & 8.84 & 2.79 & N/A & 101.8 \\
\hline xl_5 & 67.98 & 20.07 & 0.24 & 0.000 & 0.70 & 7.04 & 2.98 & $\mathrm{~N} / \mathrm{A}$ & 99.0 \\
\hline xl_6 & 69.35 & 20.19 & 0.27 & 0.000 & 0.77 & 8.81 & 2.84 & N/A & 102.2 \\
\hline xl_7 & 67.79 & 20.62 & 0.24 & 0.000 & 0.90 & 8.07 & 2.87 & $\mathrm{~N} / \mathrm{A}$ & 100.5 \\
\hline \multicolumn{10}{|l|}{ PFC-1513 } \\
\hline xl_1a & 62.67 & 20.12 & 0.26 & 0.004 & 0.89 & 8.47 & 2.78 & 0.97 & 96.2 \\
\hline $\mathrm{xl} \_1 \mathrm{~b}$ & 61.81 & 19.86 & 0.19 & 0.000 & 0.89 & 8.18 & 2.69 & 0.87 & 94.5 \\
\hline $\mathrm{xl} \_2 \mathrm{a}$ & 62.35 & 20.13 & 0.23 & 0.002 & 0.86 & 8.44 & 2.84 & 0.98 & 95.8 \\
\hline $\mathrm{xl} \_2 \mathrm{~b}$ & 62.67 & 20.23 & 0.27 & 0.000 & 0.75 & 8.39 & 2.77 & 0.96 & 96.0 \\
\hline $\mathrm{xl} \_3 \mathrm{a}$ & 63.34 & 20.26 & 0.27 & 0.000 & 0.89 & 8.50 & 2.62 & 0.89 & 96.8 \\
\hline $\mathrm{xl} \_3 \mathrm{~b}$ & 63.01 & 19.91 & 0.23 & 0.009 & 0.86 & 8.31 & 2.75 & 0.88 & 96.0 \\
\hline $\mathrm{xl} \_4 \mathrm{a}$ & 64.02 & 20.38 & 0.27 & 0.000 & 0.87 & 8.48 & 2.70 & 0.86 & 97.6 \\
\hline
\end{tabular}


Table C2 continued.

\begin{tabular}{|c|c|c|c|c|c|c|c|c|c|}
\hline $\begin{array}{l}\text { Sample and } \\
\text { crystal } \\
\text { number** }\end{array}$ & $\mathrm{SiO}_{2}$ & $\mathrm{Al}_{2} \mathrm{O}_{3}$ & $\mathrm{FeO}^{*}$ & MgO & $\mathrm{CaO}$ & $\mathrm{Na}_{2} \mathrm{O}$ & $\mathbf{K}_{2} \mathbf{O}$ & $\mathbf{B a O}$ & Total \\
\hline \multicolumn{10}{|l|}{ PFC-1513 cont. } \\
\hline $\mathrm{xl} \_4 \mathrm{~b}$ & 64.60 & 20.52 & 0.17 & 0.000 & 1.01 & 8.54 & 2.63 & 0.97 & 98.4 \\
\hline $\mathrm{xl} \_5 \mathrm{a}$ & 63.68 & 20.18 & 0.19 & 0.000 & 0.73 & 8.40 & 2.92 & 1.02 & 97.1 \\
\hline $\mathrm{xl} \_5 \mathrm{~b}$ & 63.19 & 20.17 & 0.24 & 0.000 & 0.89 & 8.38 & 2.81 & 1.01 & 96.7 \\
\hline xl_6a & 63.97 & 20.03 & 0.36 & 0.004 & 0.66 & 8.31 & 2.92 & 0.97 & 97.2 \\
\hline $\mathrm{xl} \_6 \mathrm{~b}$ & 64.15 & 20.00 & 0.24 & 0.000 & 0.73 & 8.51 & 2.86 & 0.95 & 97.5 \\
\hline $\mathrm{xl} \_7 \mathrm{a}$ & 63.69 & 20.25 & 0.24 & 0.004 & 0.95 & 8.20 & 2.76 & 0.92 & 97.0 \\
\hline $\mathrm{xl} \_7 \mathrm{~b}$ & 64.54 & 20.13 & 0.18 & 0.000 & 0.80 & 8.35 & 2.90 & 0.87 & 97.8 \\
\hline $\mathrm{xl} \_8 \mathrm{a}$ & 64.55 & 20.28 & 0.18 & 0.000 & 0.93 & 8.48 & 2.76 & 0.92 & 98.1 \\
\hline $\mathrm{xl} \_8 \mathrm{~b}$ & 62.92 & 19.41 & 0.27 & 0.000 & 0.72 & 8.28 & 2.88 & 0.77 & 95.2 \\
\hline xl_9a & 64.76 & 19.96 & 0.20 & 0.000 & 0.86 & 8.49 & 2.79 & 0.84 & 97.9 \\
\hline xl_9b & 63.91 & 20.36 & 0.18 & 0.000 & 0.88 & 8.52 & 2.76 & 0.92 & 97.5 \\
\hline xl_10a & 63.90 & 20.06 & 0.28 & 0.000 & 0.91 & 8.25 & 2.78 & 1.05 & 97.2 \\
\hline $\mathrm{xl} \_10 \mathrm{~b}$ & 63.78 & 20.28 & 0.21 & 0.000 & 0.90 & 8.34 & 2.76 & 1.02 & 97.3 \\
\hline
\end{tabular}

** Letters following crystal number indicate multiple analysis points per crystal.

1. Normalized values. 
Table C3. Unit 3 end member composition by sample.

\begin{tabular}{|c|c|c|c|}
\hline Sample and crystal number** & $\mathbf{A n ^ { 2 }}$ & $\mathbf{A b}^{\mathbf{3}}$ & Or $^{4}$ \\
\hline \multicolumn{4}{|l|}{ MS-12-33.1-a } \\
\hline xl_2 & 4.56 & 79.41 & 16.04 \\
\hline xl_6 & 4.70 & 78.37 & 16.95 \\
\hline \multicolumn{4}{|l|}{ MS-12-33.1-b } \\
\hline $\mathrm{xl} \_1 \mathrm{a}$ & 3.65 & 78.77 & 17.60 \\
\hline $\mathrm{xl} \_1 \mathrm{~b}$ & 3.66 & 77.92 & 18.44 \\
\hline $\mathrm{xl} \_2 \mathrm{a}$ & 1.85 & 78.70 & 19.46 \\
\hline $\mathrm{xl} \_2 \mathrm{~b}$ & 3.33 & 80.31 & 16.38 \\
\hline $\mathrm{xl} \_3 \mathrm{a}$ & 7.65 & 78.96 & 13.41 \\
\hline $\mathrm{xl} \_3 \mathrm{~b}$ & 4.39 & 79.46 & 16.16 \\
\hline $\mathrm{xl} \_4 \mathrm{a}$ & 3.54 & 78.60 & 17.88 \\
\hline $\mathrm{xl} \_4 \mathrm{~b}$ & 4.02 & 77.43 & 18.58 \\
\hline $\mathrm{xl} \_5 \mathrm{a}$ & 5.49 & 78.81 & 15.72 \\
\hline $\mathrm{xl} \_5 \mathrm{~b}$ & 4.63 & 79.10 & 16.28 \\
\hline xl_6a & 3.77 & 79.00 & 17.25 \\
\hline $\mathrm{xl} \_6 \mathrm{~b}$ & 3.32 & 78.31 & 18.38 \\
\hline $\mathrm{xl} \_8 \mathrm{a}$ & 4.58 & 77.81 & 17.62 \\
\hline $\mathrm{xl} \_8 \mathrm{~b}$ & 4.76 & 78.02 & 17.24 \\
\hline xl_9a & 4.44 & 79.01 & 16.56 \\
\hline $\mathrm{xl} \_9 \mathrm{~b}$ & 4.42 & 78.42 & 17.18 \\
\hline $\mathrm{xl} \_10 \mathrm{a}$ & 3.72 & 77.73 & 18.57 \\
\hline xl_10b & 3.31 & 78.70 & 18.01 \\
\hline \multicolumn{4}{|l|}{ EBC12-074 } \\
\hline xl_1a & 4.79 & 79.13 & 16.10 \\
\hline $\mathrm{xl} \_1 \mathrm{~b}$ & 4.90 & 78.41 & 16.71 \\
\hline $\mathrm{xl} \_2 \mathrm{a}$ & 4.80 & 79.15 & 16.07 \\
\hline $\mathrm{xl} \_2 \mathrm{~b}$ & 4.22 & 80.15 & 15.64 \\
\hline$x l \_3 a$ & 4.89 & 78.30 & 16.83 \\
\hline$x l \_3 b$ & 3.88 & 78.91 & 17.22 \\
\hline $\mathrm{xl} \_4 \mathrm{a}$ & 4.85 & 78.66 & 16.50 \\
\hline $\mathrm{xl} \_4 \mathrm{~b}$ & 4.53 & 78.98 & 16.51 \\
\hline xl_5a & 4.31 & 78.93 & 16.77 \\
\hline $\mathrm{xl} \_5 \mathrm{~b}$ & 4.67 & 78.86 & 16.49 \\
\hline xl_6a & 4.02 & 78.55 & 17.45 \\
\hline xl_6b & 4.31 & 78.52 & 17.18 \\
\hline xl_7a & 2.65 & 79.73 & 17.63 \\
\hline $\mathrm{xl} \_7 \mathrm{~b}$ & 2.52 & 81.95 & 15.55 \\
\hline xl_8a & 5.74 & 79.10 & 15.17 \\
\hline $\mathrm{xl} \_8 \mathrm{~b}$ & 5.87 & 79.49 & 14.66 \\
\hline xl_9a & 5.13 & 78.78 & 16.10 \\
\hline
\end{tabular}


Table C3 continued.

\begin{tabular}{|c|c|c|c|}
\hline Sample and crystal number** & $\mathbf{A n}^{2}$ & $\mathbf{A b ^ { 3 }}$ & Or $^{4}$ \\
\hline \multicolumn{4}{|l|}{ EBC12-074 cont. } \\
\hline $\mathrm{xl} \_9 \mathrm{~b}$ & 4.78 & 79.15 & 16.09 \\
\hline $\mathrm{xl} \_10 \mathrm{a}$ & 4.00 & 78.47 & 17.55 \\
\hline $\mathrm{xl} \_10 \mathrm{~b}$ & 2.74 & 78.31 & 18.97 \\
\hline \multicolumn{4}{|l|}{ MS-14-12 } \\
\hline $\mathrm{xl} \_1 \mathrm{a}$ & 3.42 & 79.11 & 17.49 \\
\hline $\mathrm{xl} \_1 \mathrm{~b}$ & 3.28 & 78.56 & 18.18 \\
\hline $\mathrm{xl} \_2 \mathrm{a}$ & 4.10 & 79.08 & 16.84 \\
\hline $\mathrm{xl} \_2 \mathrm{~b}$ & 4.25 & 79.58 & 16.18 \\
\hline xl_3a & 3.03 & 78.88 & 18.11 \\
\hline $\mathrm{xl} \_3 \mathrm{~b}$ & 3.76 & 78.85 & 17.41 \\
\hline $\mathrm{xl} \_4 \mathrm{a}$ & 3.83 & 79.36 & 16.83 \\
\hline $\mathrm{xl} \_4 \mathrm{~b}$ & 4.01 & 78.63 & 17.38 \\
\hline $\mathrm{xl} \_5 \mathrm{a}$ & 3.12 & 79.82 & 17.08 \\
\hline $\mathrm{xl} \_5 \mathrm{~b}$ & 3.11 & 78.91 & 17.99 \\
\hline xl_6a & 4.11 & 78.48 & 17.43 \\
\hline xl_6b & 3.42 & 79.38 & 17.21 \\
\hline $\mathrm{xl} \_7 \mathrm{a}$ & 4.18 & 79.32 & 16.51 \\
\hline $\mathrm{xl} \_7 \mathrm{~b}$ & 3.92 & 78.82 & 17.28 \\
\hline xl_8a & 4.34 & 79.16 & 16.51 \\
\hline $\mathrm{xl} \_8 \mathrm{~b}$ & 4.51 & 79.10 & 16.41 \\
\hline xl_9a & 4.94 & 79.26 & 15.81 \\
\hline xl_9b & 4.35 & 79.33 & 16.33 \\
\hline $\mathrm{xl} \_10 \mathrm{a}$ & 4.31 & 80.24 & 15.46 \\
\hline $\mathrm{xl} \_10 \mathrm{~b}$ & 4.20 & 78.77 & 17.05 \\
\hline \multicolumn{4}{|l|}{ MS-13-08 } \\
\hline $\mathrm{xl} \_1 \mathrm{a}$ & 3.98 & 71.76 & 24.28 \\
\hline $\mathrm{xl} \_1 \mathrm{~b}$ & 3.48 & 72.85 & 23.69 \\
\hline $\mathrm{xl} \_2 \mathrm{a}$ & 3.31 & 73.04 & 23.67 \\
\hline $\mathrm{xl} \_2 \mathrm{~b}$ & 3.17 & 73.17 & 23.68 \\
\hline $\mathrm{xl} \_2 \mathrm{c}$ & 3.25 & 73.53 & 23.24 \\
\hline xl_3a & 4.05 & 72.98 & 22.99 \\
\hline $\mathrm{xl} \_3 b$ & 4.11 & 73.20 & 22.71 \\
\hline $\mathrm{xl} \_3 \mathrm{c}$ & 3.49 & 73.16 & 23.37 \\
\hline xl_4a & 6.35 & 74.29 & 19.38 \\
\hline $\mathrm{xl} \_4 \mathrm{~b}$ & 6.02 & 75.27 & 18.73 \\
\hline $\mathrm{xl} \_4 \mathrm{c}$ & 6.35 & 74.62 & 19.06 \\
\hline $\mathrm{xl} \_5 \mathrm{a}$ & 3.71 & 72.69 & 23.62 \\
\hline $\mathrm{xl} \_5 \mathrm{~b}$ & 3.56 & 73.02 & 23.44 \\
\hline $\mathrm{xl} \_5 \mathrm{c}$ & 3.33 & 72.51 & 24.18 \\
\hline
\end{tabular}


Table C3 continued.

\begin{tabular}{|c|c|c|c|}
\hline Sample and crystal number** & $\overline{A \mathbf{A n}^{2}}$ & $\bar{A} \mathbf{A b ^ { 3 }}$ & $\mathrm{Or}^{4}$ \\
\hline \multicolumn{4}{|l|}{ MS-13-08 cont. } \\
\hline xl_6a & 2.95 & 72.62 & 24.45 \\
\hline xl_6b & 3.23 & 73.69 & 23.10 \\
\hline xl_6c & 3.89 & 72.76 & 23.37 \\
\hline $\mathrm{xl} \_7 \mathrm{a}$ & 5.81 & 74.76 & 19.45 \\
\hline $\mathrm{xl} \_7 \mathrm{~b}$ & 4.80 & 74.45 & 20.77 \\
\hline $\mathrm{xl} \_7 \mathrm{c}$ & 4.65 & 74.21 & 21.17 \\
\hline xl_8a & 3.30 & 73.76 & 22.97 \\
\hline $\mathrm{xl} \_8 \mathrm{~b}$ & 3.41 & 74.20 & 22.41 \\
\hline $\mathrm{xl} \_8 \mathrm{c}$ & 3.39 & 73.79 & 22.84 \\
\hline xl_9a & 4.34 & 72.48 & 23.21 \\
\hline $\mathrm{xl} \_9 \mathrm{~b}$ & 4.18 & 73.41 & 22.43 \\
\hline xl_9c & 4.41 & 72.46 & 23.16 \\
\hline $\mathrm{xl} \_10 \mathrm{a}$ & 3.46 & 72.78 & 23.78 \\
\hline $\mathrm{xl} \_10 \mathrm{~b}$ & 3.48 & 72.26 & 24.29 \\
\hline xl_10c & 3.43 & 72.29 & 24.30 \\
\hline \multicolumn{4}{|l|}{ MS-10-26 } \\
\hline xl_2 & 3.54 & 72.05 & 24.44 \\
\hline \multicolumn{4}{|l|}{ MS-10-25 } \\
\hline xl_1 & 4.46 & 76.98 & 18.58 \\
\hline $\mathrm{xl} \_2$ & 4.80 & 78.10 & 17.12 \\
\hline xl_3 & 4.38 & 76.43 & 19.21 \\
\hline xl_4 & 4.49 & 79.04 & 16.49 \\
\hline xl_5 & 4.15 & 74.93 & 20.94 \\
\hline xl_6 & 3.84 & 79.26 & 16.92 \\
\hline xl_7 & 4.76 & 77.13 & 18.13 \\
\hline \multicolumn{4}{|l|}{ PFC-1513 } \\
\hline xl_1a & 4.55 & 78.43 & 17.04 \\
\hline $\mathrm{xl} \_1 \mathrm{~b}$ & 4.73 & 78.28 & 17.01 \\
\hline $\mathrm{xl} \_2 \mathrm{a}$ & 4.43 & 78.19 & 17.39 \\
\hline $\mathrm{xl} \_2 \mathrm{~b}$ & 3.89 & 78.92 & 17.21 \\
\hline xl_3a & 4.60 & 79.29 & 16.13 \\
\hline $\mathrm{xl} \_3 \mathrm{~b}$ & 4.52 & 78.35 & 17.15 \\
\hline xl_4a & 4.47 & 78.95 & 16.59 \\
\hline $\mathrm{xl} \_4 \mathrm{~b}$ & 5.15 & 78.80 & 16.06 \\
\hline xl_5a & 3.78 & 78.26 & 17.98 \\
\hline $\mathrm{xl} \_5 \mathrm{~b}$ & 4.61 & 78.11 & 17.29 \\
\hline xl_6a & 3.43 & 78.35 & 18.23 \\
\hline xl_6b & 3.74 & 78.75 & 17.52 \\
\hline xl_7a & 4.99 & 77.72 & 17.31 \\
\hline
\end{tabular}


Table C3 continued.

\begin{tabular}{cccc}
\hline \hline Sample and crystal number** & $\mathbf{A n}^{\mathbf{2}}$ & $\mathbf{A b}^{\mathbf{3}}$ & $\mathbf{O r}^{\mathbf{4}}$ \\
\cline { 1 - 2 } PFC-1513 cont. & & & \\
\cline { 1 - 3 } xl_7b & 4.12 & 78.01 & 17.88 \\
xl_8a & 4.76 & 78.38 & 16.88 \\
xl_8b & 3.76 & 78.24 & 18.02 \\
xl_9a & 4.42 & 78.53 & 17.06 \\
xl_9b & 4.53 & 78.64 & 16.84 \\
xl_10a & 4.76 & 77.90 & 17.35 \\
xl_10b & 4.70 & 78.19 & 17.12 \\
\hline
\end{tabular}

** Letters following crystal number indicate multiple analysis points per crystal.

1. Normalized values

2. $\mathrm{An}=(\mathrm{CaO} * 0.714 / 40) /\left(\mathrm{CaO} * 0.714 / 40+\mathrm{Na}_{2} \mathrm{O} * 0.74 / 23+\mathrm{K}_{2} \mathrm{O} * 0.83 / 39\right) * 100$

3. $\mathrm{Ab}=\left(\mathrm{Na}_{2} \mathrm{O} * 0.74 / 23\right) /\left(\mathrm{CaO} * 0.714 / 40+\mathrm{Na}_{2} \mathrm{O} * 0.74 / 23+\mathrm{K}_{2} \mathrm{O} * 0.83 / 39\right) * 100$

4. $\mathrm{Or}=\left(\mathrm{K}_{2} \mathrm{O} * 0.83 / 39\right) /\left(\mathrm{CaO} * 0.714 / 40+\mathrm{Na}_{2} \mathrm{O} * 0.74 / 23+\mathrm{K}_{2} \mathrm{O} * 0.83 / 39\right) * 100$ 


\section{APPENDIX D: UNIT 4 CHEMICAL DATA, STANDARD DEVIATIONS AND GPS COORDINATES}

Table D1. Average feldspar end member component composition and GPS coordinates by sample.

\begin{tabular}{lccccccccc}
\hline \hline Sample & $\begin{array}{c}\text { Number } \\
\text { of } \\
\text { Crystals }\end{array}$ & An & Ab & Or & $\begin{array}{c}\text { An } \\
\text { Std. }\end{array}$ & $\begin{array}{c}\text { Al } \\
\text { Std. }\end{array}$ & $\begin{array}{c}\text { Or } \\
\text { Std. }\end{array}$ & Latitude & Longitude \\
\hline MS-12-20 & 7 & 3.9 & 78.8 & 17.3 & 0.7 & 0.6 & 0.4 & 44.4843 & -118.0869 \\
MS-15-18-2T & 3 & 5.6 & 72.8 & 21.6 & 0.2 & 0.9 & 0.7 & 43.6988 & -118.8014 \\
MS-15-7c & 2 & 3.0 & 70.3 & 26.7 & 0.1 & 2.2 & 2.3 & 43.7241 & -118.8013 \\
DIT@Craft & 10 & 4.2 & 78.1 & 17.7 & 0.3 & 2.1 & 2.0 & 43.7883 & -118.7550 \\
Point & 10 & 4.0 & 77.3 & 18.7 & 0.7 & 0.6 & 0.4 & 43.7799 & -118.0233 \\
MS-15-20a & & & & & & & & & \\
\hline
\end{tabular}


Table D2. Unit 4 EMP major element feldspar chemical data by sample.

\begin{tabular}{|c|c|c|c|c|c|c|c|c|c|}
\hline $\begin{array}{l}\text { Sample and } \\
\text { crystal } \\
\text { number** }\end{array}$ & $\mathrm{SiO}_{2}$ & $\mathrm{Al}_{2} \mathrm{O}_{3}$ & $\mathrm{FeO}^{*}$ & MgO & $\mathrm{CaO}$ & $\mathrm{Na}_{2} \mathrm{O}$ & $\mathrm{K}_{2} \mathrm{O}$ & $\mathrm{BaO}$ & Total \\
\hline \multicolumn{10}{|l|}{ MS-12-20 } \\
\hline xl_1a & 68.05 & 20.79 & 0.21 & 0.008 & 0.76 & 8.73 & 2.94 & 1.04 & 102.5 \\
\hline $\mathrm{xl} \_1 \mathrm{~b}$ & 68.26 & 20.62 & 0.22 & 0.000 & 0.64 & 8.68 & 2.96 & 1.02 & 102.4 \\
\hline $\mathrm{xl} \_2 \mathrm{a}$ & 67.53 & 20.49 & 0.20 & 0.002 & 0.88 & 8.65 & 2.75 & 0.96 & 101.5 \\
\hline $\mathrm{xl} \_2 \mathrm{~b}$ & 67.84 & 20.36 & 0.20 & 0.006 & 0.87 & 8.69 & 2.82 & 1.01 & 101.8 \\
\hline xl_3a & 67.78 & 20.81 & 0.20 & 0.000 & 0.96 & 8.76 & 2.95 & 1.14 & 102.6 \\
\hline$x l \_3 b$ & 67.27 & 20.25 & 0.17 & 0.000 & 0.82 & 8.62 & 2.86 & 1.07 & 101.1 \\
\hline xl_5a & 68.98 & 20.87 & 0.21 & 0.008 & 0.88 & 8.72 & 2.78 & 0.95 & 103.4 \\
\hline $\mathrm{xl} \_5 \mathrm{~b}$ & 70.73 & 20.20 & 0.22 & 0.002 & 0.54 & 9.00 & 2.92 & 0.80 & 104.4 \\
\hline xl_6a & 69.06 & 20.66 & 0.20 & 0.001 & 0.88 & 8.57 & 2.83 & 1.03 & 103.2 \\
\hline xl_6b & 68.79 & 20.10 & 0.23 & 0.004 & 0.65 & 8.59 & 2.93 & 0.92 & 102.2 \\
\hline xl_7a & 69.47 & 20.36 & 0.16 & 0.000 & 0.62 & 8.69 & 2.97 & 0.84 & 103.1 \\
\hline $\mathrm{xl} \_7 \mathrm{~b}$ & 68.43 & 20.55 & 0.22 & 0.000 & 0.86 & 8.44 & 2.91 & 1.09 & 102.5 \\
\hline \multicolumn{10}{|l|}{ MS-15-18-2T } \\
\hline xl_3 & 68.01 & 20.29 & 0.27 & 0.000 & 0.87 & 8.77 & 2.82 & N/A & 101.0 \\
\hline xl_6 & 66.68 & 20.41 & 0.21 & 0.000 & 0.87 & 7.94 & 2.82 & N/A & 98.9 \\
\hline xl_7 & 67.88 & 20.64 & 0.21 & 0.000 & 0.89 & 8.51 & 2.87 & N/A & 101.0 \\
\hline \multicolumn{10}{|l|}{ MS-15-7c } \\
\hline xl_2 & 67.56 & 19.78 & 0.18 & 0.001 & 0.58 & 7.61 & 4.01 & N/A & 99.7 \\
\hline xl_5 & 68.01 & 19.58 & 0.20 & 0.006 & 0.58 & 7.44 & 4.63 & N/A & 100.5 \\
\hline \multicolumn{10}{|l|}{$\begin{array}{l}\text { DIT@Craft } \\
\text { Point }\end{array}$} \\
\hline xl_1a & 66.16 & 20.43 & 0.16 & 0.000 & 0.90 & 8.41 & 2.78 & 1.04 & 99.9 \\
\hline $\mathrm{xl} \_1 \mathrm{~b}$ & 66.73 & 20.32 & 0.21 & 0.004 & 0.87 & 8.56 & 2.78 & 1.03 & 100.5 \\
\hline $\mathrm{xl} \_2 \mathrm{a}$ & 67.32 & 20.56 & 0.21 & 0.000 & 0.92 & 8.54 & 2.84 & 1.05 & 101.5 \\
\hline $\mathrm{xl} \_2 \mathrm{~b}$ & 66.94 & 20.61 & 0.23 & 0.000 & 0.81 & 8.61 & 2.90 & 1.09 & 101.2 \\
\hline$x l \_3 a$ & 66.04 & 20.61 & 0.18 & 0.000 & 0.81 & 8.54 & 2.87 & 1.11 & 100.2 \\
\hline $\mathrm{xl} \_3 \mathrm{~b}$ & 67.56 & 20.45 & 0.21 & 0.000 & 0.78 & 8.49 & 2.91 & 1.04 & 101.4 \\
\hline xl_4a & 65.32 & 20.34 & 0.17 & 0.004 & 0.87 & 8.40 & 2.78 & 1.10 & 99.0 \\
\hline$x l \_4 b$ & 66.18 & 20.65 & 0.21 & 0.001 & 0.95 & 8.56 & 2.67 & 0.95 & 100.2 \\
\hline xl_5a & 65.72 & 20.67 & 0.19 & 0.000 & 0.81 & 8.61 & 2.95 & 1.10 & 100.0 \\
\hline $\mathrm{xl} \_5 \mathrm{~b}$ & 66.83 & 20.29 & 0.22 & 0.000 & 0.80 & 8.63 & 2.96 & 1.16 & 100.9 \\
\hline xl_6a & 67.49 & 20.76 & 0.23 & 0.003 & 0.89 & 8.57 & 2.75 & 0.96 & 101.7 \\
\hline xl_6b & 67.35 & 20.64 & 0.20 & 0.000 & 0.91 & 8.73 & 2.83 & 0.94 & 101.6 \\
\hline xl_7a & 67.76 & 20.62 & 0.20 & 0.000 & 0.74 & 8.66 & 2.98 & 1.04 & 102.0 \\
\hline xl_7b & 66.25 & 20.39 & 0.27 & 0.001 & 0.92 & 7.47 & 4.26 & 1.00 & 100.6 \\
\hline xl_8a & 66.86 & 20.42 & 0.23 & 0.000 & 0.75 & 8.85 & 2.76 & 0.89 & 100.8 \\
\hline xl_8b & 66.16 & 20.51 & 0.17 & 0.000 & 0.82 & 8.57 & 2.87 & 1.02 & 100.1 \\
\hline xl_9a & 66.51 & 20.19 & 0.19 & 0.000 & 0.77 & 8.44 & 2.98 & 1.04 & 100.1 \\
\hline
\end{tabular}


Table D2 continued.

\begin{tabular}{|c|c|c|c|c|c|c|c|c|c|}
\hline $\begin{array}{l}\text { Sample and } \\
\text { crystal } \\
\text { number** }\end{array}$ & $\mathrm{SiO}_{2}$ & $\mathrm{Al}_{2} \mathrm{O}_{3}$ & $\mathrm{FeO}^{*}$ & MgO & $\mathrm{CaO}$ & $\mathrm{Na}_{2} \mathrm{O}$ & $\mathbf{K}_{2} \mathbf{O}$ & $\mathrm{BaO}$ & Total \\
\hline \multicolumn{10}{|l|}{$\begin{array}{l}\text { DIT@Craft } \\
\text { Point cont. }\end{array}$} \\
\hline xl_9b & 66.95 & 20.22 & 0.24 & 0.000 & 0.80 & 8.67 & 2.89 & 1.11 & 100.9 \\
\hline xl_10a & 68.62 & 20.27 & 0.18 & 0.001 & 0.82 & 8.85 & 2.84 & 1.01 & 102.6 \\
\hline $\mathrm{xl} \_10 \mathrm{~b}$ & 67.11 & 20.66 & 0.13 & 0.000 & 0.77 & 8.56 & 2.88 & 1.03 & 101.2 \\
\hline \multicolumn{10}{|l|}{ MS-15-20a } \\
\hline xl_1a & 67.24 & 20.36 & 0.22 & 0.000 & 0.82 & 8.54 & 3.23 & 1.11 & 101.5 \\
\hline $\mathrm{xl} \_1 \mathrm{~b}$ & 66.79 & 20.43 & 0.26 & 0.000 & 0.84 & 8.55 & 3.49 & 1.09 & 101.4 \\
\hline $\mathrm{xl} \_2 \mathrm{a}$ & 67.26 & 20.63 & 0.23 & 0.000 & 0.91 & 8.93 & 2.88 & 0.96 & 101.8 \\
\hline $\mathrm{xl} \_2 \mathrm{~b}$ & 67.57 & 20.75 & 0.16 & 0.000 & 0.91 & 8.86 & 2.74 & 1.02 & 102.0 \\
\hline xl_3a & 66.87 & 20.92 & 0.29 & 0.000 & 1.01 & 8.90 & 2.70 & 1.03 & 100.0 \\
\hline$x l \_3 b$ & 65.91 & 20.36 & 0.22 & 0.000 & 0.95 & 8.78 & 2.80 & 0.94 & 101.4 \\
\hline xl_4a & 67.16 & 20.49 & 0.15 & 0.000 & 0.95 & 8.84 & 2.75 & 1.01 & 101.0 \\
\hline $\mathrm{xl} \_4 \mathrm{~b}$ & 66.50 & 20.82 & 0.20 & 0.000 & 0.96 & 8.79 & 2.72 & 1.01 & 101.2 \\
\hline xl_5a & 67.36 & 20.38 & 0.26 & 0.000 & 0.79 & 8.63 & 2.92 & 0.84 & 103.1 \\
\hline xl_5b & 68.14 & 20.84 & 0.24 & 0.000 & 0.83 & 9.27 & 2.95 & 0.88 & 101.2 \\
\hline xl_6a & 66.79 & 20.54 & 0.21 & 0.006 & 0.85 & 9.01 & 2.81 & 0.94 & 101.0 \\
\hline xl_6b & 66.58 & 20.83 & 0.24 & 0.000 & 0.94 & 8.69 & 2.74 & 0.99 & 101.2 \\
\hline xl_7a & 67.41 & 19.78 & 0.26 & 0.000 & 0.14 & 8.10 & 5.11 & 0.39 & 100.9 \\
\hline xl_7b & 67.17 & 19.83 & 0.25 & 0.000 & 0.19 & 8.09 & 5.02 & 0.34 & 101.4 \\
\hline xl_8a & 66.25 & 20.16 & 0.23 & 0.000 & 1.03 & 8.32 & 2.69 & 1.04 & 99.7 \\
\hline xl_8b & 66.92 & 20.89 & 0.22 & 0.000 & 0.99 & 8.67 & 2.69 & 0.99 & 101.4 \\
\hline xl_9a & 67.13 & 20.43 & 0.27 & 0.000 & 0.86 & 8.49 & 3.48 & 0.93 & 101.6 \\
\hline xl_9b & 67.07 & 20.50 & 0.25 & 0.000 & 0.87 & 8.25 & 3.82 & 0.91 & 101.7 \\
\hline xl_10a & 66.83 & 20.28 & 0.26 & 0.000 & 0.61 & 8.81 & 2.95 & 1.00 & 100.7 \\
\hline xl_10b & 66.32 & 20.28 & 0.26 & 0.000 & 0.72 & 8.94 & 3.08 & 1.04 & 100.6 \\
\hline
\end{tabular}


Table D3. Unit 4 end member compositions by sample.

\begin{tabular}{|c|c|c|c|}
\hline Sample and crystal number** & $A n^{2}$ & $\mathbf{A b ^ { 3 }}$ & $\mathrm{Or}^{4}$ \\
\hline \multicolumn{4}{|l|}{ MS-12-20 } \\
\hline xl_1a & 3.82 & 78.70 & 17.50 \\
\hline $\mathrm{xl} \_1 \mathrm{~b}$ & 3.25 & 78.94 & 17.82 \\
\hline $\mathrm{xl} \_2 \mathrm{a}$ & 4.47 & 78.95 & 16.59 \\
\hline $\mathrm{xl} \_2 \mathrm{~b}$ & 4.39 & 78.74 & 16.89 \\
\hline xl_3a & 4.73 & 77.93 & 17.36 \\
\hline $\mathrm{xl} \_3 \mathrm{~b}$ & 4.16 & 78.59 & 17.27 \\
\hline xl_5a & 4.44 & 78.91 & 16.66 \\
\hline $\mathrm{xl} \_5 \mathrm{~b}$ & 2.65 & 80.15 & 17.21 \\
\hline xl_6a & 4.47 & 78.40 & 17.14 \\
\hline xl_6b & 3.32 & 78.91 & 17.79 \\
\hline xl_7a & 3.15 & 78.98 & 17.89 \\
\hline xl_7b & 4.42 & 77.87 & 17.72 \\
\hline \multicolumn{4}{|l|}{ MS-15-18-2T } \\
\hline xl_3 & 4.33 & 78.92 & 16.76 \\
\hline xl_6 & 4.71 & 77.20 & 18.11 \\
\hline xl_7 & 4.51 & 78.10 & 17.41 \\
\hline \multicolumn{4}{|l|}{ MS-15-7c } \\
\hline xl_2 & 3.04 & 71.94 & 25.04 \\
\hline xl_5 & 2.96 & 68.77 & 28.29 \\
\hline \multicolumn{4}{|l|}{ DIT@Craft Point } \\
\hline $\mathrm{xl} \_1 \mathrm{a}$ & 4.63 & 78.26 & 17.12 \\
\hline $\mathrm{xl} \_1 \mathrm{~b}$ & 4.45 & 78.66 & 16.91 \\
\hline $\mathrm{xl} \_2 \mathrm{a}$ & 4.67 & 78.16 & 17.19 \\
\hline $\mathrm{xl} \_2 \mathrm{~b}$ & 4.12 & 78.41 & 17.49 \\
\hline xl_3a & 4.11 & 78.47 & 17.44 \\
\hline$x l \_3 b$ & 3.97 & 78.28 & 17.76 \\
\hline xl_4a & 4.50 & 78.34 & 17.17 \\
\hline $\mathrm{xl} \_4 \mathrm{~b}$ & 4.87 & 78.87 & 16.27 \\
\hline xl_5a & 4.08 & 78.24 & 17.70 \\
\hline xl_5b & 4.01 & 78.26 & 17.75 \\
\hline xl_6a & 4.55 & 78.78 & 16.69 \\
\hline xl_6b & 4.53 & 78.65 & 16.84 \\
\hline xl_7a & 3.70 & 78.49 & 17.83 \\
\hline xl_7b & 4.75 & 69.18 & 26.09 \\
\hline xl_8a & 3.78 & 79.77 & 16.47 \\
\hline $\mathrm{xl} \_8 \mathrm{~b}$ & 4.17 & 78.47 & 17.38 \\
\hline xl_9a & 3.97 & 77.89 & 18.16 \\
\hline xl_9b & 4.00 & 78.67 & 17.34 \\
\hline
\end{tabular}


Table D3 continued.

\begin{tabular}{cccc}
\hline \hline Sample and crystal number** & $\mathbf{A n}^{\mathbf{2}}$ & $\mathbf{A b}^{\mathbf{3}}$ & $\mathbf{O r}^{\mathbf{4}}$ \\
\hline DIT@Craft Point cont. & & & \\
\cline { 1 - 3 } xl_10a & 4.07 & 79.13 & 16.82 \\
xl_10b & 3.94 & 78.57 & 17.50 \\
MS-12-20 & & & \\
\hline xl_1a & 4.06 & 76.75 & 19.20 \\
xl_1b & 4.11 & 75.53 & 20.38 \\
xl_2a & 4.44 & 78.76 & 16.82 \\
xl_2b & 4.50 & 79.32 & 16.20 \\
xl_3a & 4.96 & 79.19 & 15.86 \\
xl_3b & 4.72 & 78.71 & 16.59 \\
xl_4a & 4.71 & 79.03 & 16.28 \\
xl_4b & 4.80 & 79.06 & 16.16 \\
xl_5a & 3.99 & 78.47 & 17.56 \\
xl_5b & 3.92 & 79.39 & 16.70 \\
xl_6a & 4.15 & 79.49 & 16.38 \\
xl_6b & 4.72 & 78.84 & 16.46 \\
xl_7a & 0.68 & 70.09 & 29.26 \\
xl_7b & 0.91 & 70.30 & 28.82 \\
xl_8a & 5.34 & 77.98 & 16.70 \\
xl_8b & 5.00 & 78.87 & 16.15 \\
xl_9a & 4.21 & 75.40 & 20.41 \\
xl_9b & 3.53 & 73.29 & 22.46 \\
xl_10a & 79.41 & 17.58 \\
xl_10b & 78.59 & 17.90 \\
\hline
\end{tabular}

** Letters following crystal number indicate multiple analysis points per crystal.

1. Normalized values.

2. $\mathrm{An}=(\mathrm{CaO} * 0.714 / 40) /\left(\mathrm{CaO} * 0.714 / 40+\mathrm{Na}_{2} \mathrm{O} * 0.74 / 23+\mathrm{K}_{2} \mathrm{O} * 0.83 / 39\right) * 100$

3. $\mathrm{Ab}=\left(\mathrm{Na}_{2} \mathrm{O} * 0.74 / 23\right) /\left(\mathrm{CaO} * 0.714 / 40+\mathrm{Na}_{2} \mathrm{O} * 0.74 / 23+\mathrm{K}_{2} \mathrm{O} * 0.83 / 39\right) * 100$

4. $\mathrm{Or}=\left(\mathrm{K}_{2} \mathrm{O} * 0.83 / 39\right) /\left(\mathrm{CaO} * 0.714 / 40+\mathrm{Na}_{2} \mathrm{O} * 0.74 / 23+\mathrm{K}_{2} \mathrm{O} * 0.83 / 39\right) * 100$ 


\section{APPENDIX E: NON-UNIT DESIGNATING FELDSPAR CHEMICAL DATA}

Table E1. Non-unit designating EMP major element chemical data by feldspar type.

\begin{tabular}{|c|c|c|c|c|c|c|c|c|c|c|}
\hline $\begin{array}{l}\text { Feldspar type, } \\
\text { sample and } \\
\text { crystal number** }\end{array}$ & $\begin{array}{l}\text { Sample } \\
\text { Unit }\end{array}$ & $\mathrm{SiO}_{2}$ & $\mathrm{Al}_{2} \mathrm{O}_{3}$ & $\mathrm{FeO}^{*}$ & MgO & $\mathrm{CaO}$ & $\mathrm{Na}_{2} \mathrm{O}$ & $\mathbf{K}_{2} \mathbf{O}$ & $\mathrm{BaO}$ & Total \\
\hline \multicolumn{11}{|l|}{ albite } \\
\hline $\begin{array}{l}\text { MS-12-33.1_xlC1 } \\
\text { andesine }\end{array}$ & 3 & 67.28 & 20.97 & 0.25 & 0.005 & 1.43 & 9.34 & 1.57 & 0.38 & 101.2 \\
\hline MS-10-20_xl1a & 2 & 59.72 & 26.52 & 0.30 & 0.000 & 7.71 & 6.84 & 0.39 & 0.03 & 101.5 \\
\hline MS-10-20_xl1b & 2 & 61.50 & 26.29 & 0.25 & 0.000 & 6.68 & 7.32 & 0.46 & 0.10 & 102.6 \\
\hline MS-14-20a_x1a & N/A & 57.10 & 27.65 & 0.32 & 0.020 & 9.22 & 6.16 & 0.36 & 0.06 & 100.9 \\
\hline MS-14-20a_xl1b & N/A & 58.98 & 25.38 & 0.28 & 0.016 & 7.50 & 6.96 & 0.48 & 0.11 & 99.7 \\
\hline MS-12-20_xl4a & 4 & 59.72 & 26.52 & 0.30 & 0.000 & 7.71 & 6.84 & 0.39 & 0.03 & 101.5 \\
\hline MS-12-20_xl4b & 4 & 61.50 & 26.29 & 0.25 & 0.000 & 6.68 & 7.32 & 0.46 & 0.10 & 100.7 \\
\hline MS-15-12_xl2 & 2 & 61.29 & 24.68 & 0.27 & 0.006 & 6.04 & 7.27 & 0.67 & N/A & 100.2 \\
\hline MS-15-12_xl3 & 2 & 58.25 & 26.69 & 0.20 & 0.010 & 7.95 & 6.41 & 0.44 & N/A & 99.9 \\
\hline MS-15-12_xl5 & 2 & 56.32 & 27.74 & 0.11 & 0.000 & 8.76 & 6.09 & 0.20 & N/A & 99.2 \\
\hline MS-15-38_x15 & 1 & 58.64 & 26.26 & 0.21 & 0.001 & 7.94 & 6.52 & 0.31 & N/A & 99.9 \\
\hline MS-15-12.4_xl1a & 2 & 59.38 & 25.72 & 0.23 & 0.008 & 7.31 & 6.53 & 0.54 & 0.12 & 99.8 \\
\hline MS-15-12.4_xl1b & 2 & 59.19 & 25.96 & 0.22 & 0.010 & 7.80 & 6.36 & 0.51 & 0.08 & 100.1 \\
\hline MS-15-12.4_xl3a & 2 & 59.87 & 25.14 & 0.20 & 0.001 & 6.82 & 6.96 & 0.62 & 0.14 & 99.8 \\
\hline MS-15-12.4_xl3b & 2 & 61.82 & 24.51 & 0.25 & 0.000 & 5.95 & 7.18 & 0.70 & 0.11 & 100.5 \\
\hline MS-15-12.4_xl5a & 2 & 63.05 & 24.13 & 0.21 & 0.000 & 5.44 & 7.66 & 0.80 & 0.20 & 101.5 \\
\hline MS-15-12.4_xl5b & 2 & 59.88 & 25.43 & 0.19 & 0.000 & 7.35 & 6.70 & 0.57 & 0.07 & 100.2 \\
\hline MS-15-12.4_x16a & 2 & 60.78 & 24.71 & 0.17 & 0.003 & 6.15 & 7.22 & 0.64 & 0.15 & 99.8 \\
\hline MS-15-12.4_x16b & 2 & 61.46 & 24.16 & 0.21 & 0.013 & 5.49 & 7.36 & 0.75 & 0.10 & 99.5 \\
\hline MS-15-12.4_x18a & 2 & 61.12 & 24.80 & 0.24 & 0.007 & 6.39 & 6.99 & 0.58 & 0.10 & 100.2 \\
\hline MS-15-12.4_x18b & 2 & 59.46 & 25.93 & 0.22 & 0.000 & 7.55 & 6.42 & 0.51 & 0.06 & 100.2 \\
\hline MS-15-12.4 xl10a & 2 & 58.45 & 26.05 & 0.28 & 0.003 & 8.34 & 6.40 & 0.38 & 0.06 & 100.0 \\
\hline MS-15-12.4 xl10b & 2 & 58.04 & 26.85 & 0.32 & 0.003 & 8.66 & 6.01 & 0.40 & 0.06 & 100.3 \\
\hline MS-15-12.5_xlJ2a & 2 & 58.14 & 26.08 & 0.28 & 0.013 & 8.31 & 6.14 & 0.43 & 0.07 & 99.5 \\
\hline MS-15-12.5 xlJ2b & 2 & 57.64 & 26.48 & 0.27 & 0.000 & 8.46 & 6.16 & 0.39 & 0.05 & 99.4 \\
\hline MS-15-12.5_xlJ3a & 2 & 56.92 & 26.65 & 0.26 & 0.000 & 9.04 & 5.86 & 0.33 & 0.04 & 99.1 \\
\hline MS-15-12.5_xlJ3b & 2 & 58.25 & 25.98 & 0.31 & 0.001 & 7.83 & 6.24 & 0.44 & 0.04 & 99.1 \\
\hline MS-15-12.5_xlJ5a & 2 & 62.68 & 23.88 & 0.19 & 0.003 & 5.76 & 7.31 & 0.76 & 0.13 & 100.7 \\
\hline MS-15-12.5_xlJ5b & 2 & 61.35 & 25.18 & 0.25 & 0.008 & 6.60 & 7.05 & 0.56 & 0.08 & 101.1 \\
\hline MS-15-12.5 xlJ6a & 2 & 60.24 & 24.50 & 0.23 & 0.001 & 6.48 & 6.89 & 0.65 & 0.09 & 99.1 \\
\hline MS-15-12.5 xlJ6b & 2 & 61.70 & 24.73 & 0.18 & 0.005 & 6.31 & 7.17 & 0.66 & 0.09 & 100.8 \\
\hline MS-15-12.5 xlJ7a & 2 & 58.41 & 26.38 & 0.26 & 0.000 & 8.43 & 6.07 & 0.39 & 0.04 & 100.0 \\
\hline MS-15-12.5 xlJ7b & 2 & 58.38 & 26.95 & 0.25 & 0.008 & 8.59 & 6.27 & 0.39 & 0.06 & 100.9 \\
\hline MS-15-12.5 xlJ10a & 2 & 60.41 & 25.20 & 0.22 & 0.000 & 7.13 & 6.71 & 0.55 & 0.09 & 100.3 \\
\hline
\end{tabular}


Table E1 continued.

\begin{tabular}{|c|c|c|c|c|c|c|c|c|c|c|}
\hline $\begin{array}{l}\text { Feldspar type, } \\
\text { sample and } \\
\text { crystal number*** }\end{array}$ & $\begin{array}{l}\text { Sample } \\
\text { Unit }\end{array}$ & $\mathrm{SiO}_{2}$ & $\mathrm{Al}_{2} \mathrm{O}_{3}$ & $\mathrm{FeO}^{*}$ & MgO & $\mathrm{CaO}$ & $\mathrm{Na}_{2} \mathrm{O}$ & $\mathbf{K}_{2} \mathbf{O}$ & $\mathbf{B a O}$ & Total \\
\hline \multicolumn{11}{|l|}{ andesine cont. } \\
\hline MS-15-12.5 xlJ10b & 2 & 60.35 & 25.47 & 0.24 & 0.008 & 7.23 & 6.66 & 0.55 & 0.09 & 100.6 \\
\hline MS-15-12.5 xlK1a & 2 & 59.06 & 25.96 & 0.27 & 0.010 & 7.37 & 6.96 & 0.38 & 0.07 & 100.1 \\
\hline MS-15-12.5 xlK1b & 2 & 58.43 & 26.53 & 0.32 & 0.012 & 8.11 & 6.98 & 0.37 & 0.03 & 100.8 \\
\hline MS-14-4b xl1a & N/A & 54.39 & 26.86 & 0.28 & 0.023 & 8.89 & 5.77 & 0.41 & 0.05 & 96.7 \\
\hline MS-14-4b xl1b & N/A & 54.35 & 26.50 & 0.23 & 0.009 & 8.54 & 5.96 & 0.41 & 0.04 & 96.0 \\
\hline MS-15-28.1b x13a & 2 & 57.19 & 28.46 & 0.29 & 0.027 & 9.79 & 5.56 & 0.34 & 0.07 & 101.7 \\
\hline MS-15-28.1b xl3b & 2 & 56.98 & 27.18 & 0.31 & 0.029 & 9.14 & 5.82 & 0.39 & 0.11 & 100.0 \\
\hline MS-15-28.1b x16a & 2 & 59.22 & 26.30 & 0.16 & 0.016 & 8.07 & 6.38 & 0.38 & 0.04 & 100.6 \\
\hline $\begin{array}{l}\text { MS-15-28.1b xl6b } \\
\text { anorthoclase }\end{array}$ & 2 & 57.85 & 26.25 & 0.26 & 0.007 & 7.93 & 6.02 & 0.41 & 0.00 & 98.7 \\
\hline MS-10-26_x15 & 4 & 67.11 & 19.52 & 0.31 & 0.004 & 0.38 & 7.08 & 5.42 & N/A & 99.8 \\
\hline MS-12-33.1_xlC4 & 3 & 69.86 & 19.78 & 0.24 & 0.000 & 0.22 & 8.22 & 4.66 & 0.55 & 103.5 \\
\hline MS-12-33.1 xlH7a & 3 & 67.55 & 19.29 & 0.21 & 0.000 & 0.24 & 7.66 & 5.20 & 0.60 & 100.8 \\
\hline MS-12-33.1 xlH7b & 3 & 68.01 & 19.44 & 0.18 & 0.000 & 0.21 & 7.59 & 5.19 & 0.50 & 101.1 \\
\hline MS-15-28.1b_xl4a & 2 & 67.46 & 19.97 & 0.23 & 0.003 & 0.40 & 7.42 & 4.93 & 0.39 & 100.8 \\
\hline \multicolumn{10}{|l|}{ bytownite } & 101.4 \\
\hline MS-14-20a_x12a & N/A & 49.32 & 31.99 & 0.50 & 0.137 & 15.23 & 2.73 & 0.16 & 0.02 & 100.1 \\
\hline MS-14-20a_x12b & N/A & 51.73 & 29.93 & 0.53 & 0.113 & 13.27 & 3.69 & 0.18 & 0.02 & 99.5 \\
\hline MS-14-20a_xl3a & N/A & 47.22 & 33.87 & 0.50 & 0.107 & 16.71 & 1.81 & 0.06 & 0.02 & 100.3 \\
\hline MS-14-20a_xl3b & N/A & 48.90 & 32.52 & 0.52 & 0.126 & 15.77 & 2.53 & 0.08 & 0.02 & 100.5 \\
\hline MS-14-20a_xl5a & N/A & 49.48 & 32.38 & 0.41 & 0.117 & 15.20 & 2.87 & 0.12 & 0.00 & 100.6 \\
\hline MS-14-20a_xl5b & N/A & 51.32 & 30.28 & 0.47 & 0.122 & 13.55 & 3.52 & 0.21 & 0.01 & 99.5 \\
\hline MS-15-18-1T_x12a & N/A & 49.90 & 32.12 & 0.52 & 0.185 & 15.04 & 2.76 & 0.09 & N/A & 100.6 \\
\hline MS-15-18-1T xl2b & N/A & 50.12 & 31.59 & 0.53 & 0.187 & 15.01 & 2.91 & 0.10 & N/A & 100.4 \\
\hline MS-15-18-1T_xl4a & N/A & 50.78 & 32.12 & 0.46 & 0.191 & 14.91 & 3.03 & 0.08 & N/A & 101.6 \\
\hline MS-15-18-1T xl4b & N/A & 51.76 & 30.54 & 0.92 & 0.303 & 13.65 & 3.22 & 0.12 & N/A & 100.5 \\
\hline MS-15-18-1T_xl5a & N/A & 54.73 & 29.00 & 0.92 & 0.193 & 11.69 & 4.70 & 0.21 & N/A & 101.4 \\
\hline MS-15-18-1T xl5b & N/A & 50.57 & 3.54 & 10.03 & $\begin{array}{r}13.87 \\
4\end{array}$ & 19.89 & 0.31 & 0.00 & N/A & 98.2 \\
\hline MS-15-18-1T xl6a & N/A & 50.83 & 31.54 & 0.58 & 0.199 & 14.46 & 3.06 & 0.09 & N/A & 100.8 \\
\hline MS-15-18-1T xl6b & N/A & 51.87 & 30.44 & 0.54 & 0.236 & 13.87 & 3.51 & 0.11 & N/A & 100.6 \\
\hline MS-15-12.3 xl7a & 2 & 50.72 & 31.41 & 0.58 & 0.151 & 14.93 & 3.05 & 0.09 & 0.03 & 101.0 \\
\hline $\begin{array}{l}\text { MS-15-12.3 x17b } \\
\text { bytownite }\end{array}$ & 2 & 50.84 & 31.46 & 0.49 & 0.147 & 14.71 & 3.06 & 0.08 & 0.00 & 100.8 \\
\hline MS-15-18.2 xl8a & 2 & 48.58 & 31.16 & 0.48 & 0.150 & 14.02 & 3.16 & 0.11 & 0.00 & 97.7 \\
\hline MS-15-18.2 xl8b & 2 & 49.16 & 31.13 & 0.52 & 0.141 & 14.28 & 3.05 & 0.09 & 0.02 & 98.4 \\
\hline MS-15-18.2 x19a & 2 & 48.58 & 31.03 & 0.53 & 0.134 & 14.43 & 3.02 & 0.09 & 0.03 & 99.4 \\
\hline MS-15-18.2_x19b & 2 & 49.17 & 31.53 & 0.64 & 0.151 & 14.13 & 3.12 & 0.07 & 0.00 & 96.9 \\
\hline & & & & & & & & & & 118 \\
\hline
\end{tabular}


Table E1 continued.

\begin{tabular}{|c|c|c|c|c|c|c|c|c|c|c|}
\hline $\begin{array}{l}\text { Feldspar type, } \\
\text { sample and } \\
\text { crystal number** }\end{array}$ & $\begin{array}{l}\text { Sample } \\
\text { Unit }\end{array}$ & $\mathrm{SiO}_{2}$ & $\mathrm{Al}_{2} \mathrm{O}_{3}$ & $\mathrm{FeO}^{*}$ & MgO & $\mathrm{CaO}$ & $\mathrm{Na}_{2} \mathrm{O}$ & $\mathbf{K}_{2} \mathbf{O}$ & $\mathbf{B a O}$ & Total \\
\hline \multicolumn{11}{|l|}{ labradorite } \\
\hline MS-10-26_xl1 & 4 & 54.97 & 29.19 & 0.61 & 0.098 & 11.32 & 4.78 & 0.22 & N/A & 101.2 \\
\hline MS-14-20a_xl4a & N/A & 50.61 & 31.76 & 0.48 & 0.123 & 14.62 & 2.94 & 0.12 & 0.03 & 100.7 \\
\hline MS-14-20a_xl4b & N/A & 51.81 & 30.13 & 0.54 & 0.105 & 12.96 & 3.83 & 0.20 & 0.03 & 99.6 \\
\hline MS-14-20a_x16a & N/A & 53.25 & 29.73 & 0.60 & 0.111 & 12.54 & 4.32 & 0.17 & 0.03 & 99.5 \\
\hline MS-14-20a_xl6b & N/A & 51.16 & 30.36 & 0.57 & 0.121 & 13.33 & 3.91 & 0.17 & 0.00 & 100.8 \\
\hline MS-15-12.3 xl1a & 2 & 54.30 & 29.34 & 0.51 & 0.089 & 11.69 & 4.55 & 0.18 & 0.00 & 100.7 \\
\hline MS-15-12.3 xl1b & 2 & 50.20 & 31.19 & 0.60 & 0.097 & 14.01 & 3.34 & 0.14 & 0.03 & 99.6 \\
\hline MS-15-18.2 xl3a & 2 & 50.71 & 30.85 & 0.55 & 0.166 & 13.40 & 3.58 & 0.12 & 0.01 & 99.4 \\
\hline MS-15-18.2 xl3b & 2 & 48.83 & 30.07 & 0.55 & 0.146 & 13.81 & 3.37 & 0.11 & 0.00 & 96.9 \\
\hline MS-15-12.5 xlK3a & 2 & 55.89 & 28.54 & 0.35 & 0.024 & 10.31 & 5.47 & 0.26 & 0.03 & 100.9 \\
\hline MS-15-12.5 xlK3b & 2 & 54.69 & 28.39 & 0.29 & 0.024 & 10.59 & 5.31 & 0.28 & 0.01 & 99.6 \\
\hline MS-15-18-2T xl1 & 4 & 56.11 & 28.63 & 0.61 & 0.075 & 11.35 & 4.89 & 0.23 & N/A & 101.9 \\
\hline MS-15-18-1T xl3a & N/A & 55.15 & 28.49 & 0.60 & 0.086 & 10.73 & 5.22 & 0.22 & N/A & 100.5 \\
\hline MS-15-18-1T xl3b & N/A & 55.63 & 28.30 & 0.61 & 0.086 & 10.48 & 5.43 & 0.25 & N/A & 100.8 \\
\hline MS-15-28.1b x17a & 2 & 55.01 & 29.30 & 0.38 & 0.047 & 11.27 & 4.81 & 0.19 & 0.05 & 101.1 \\
\hline $\begin{array}{l}\text { MS-15-28.1b x17b } \\
\text { oligoclase }\end{array}$ & 2 & 56.08 & 28.68 & 0.38 & 0.046 & 10.67 & 5.06 & 0.27 & 0.03 & 101.2 \\
\hline MS-11-4.1_xl7a & 1 & 60.61 & 24.74 & 0.17 & 0.000 & 4.66 & 8.81 & 0.80 & 0.22 & 100.0 \\
\hline MS-11-4.1_xl7b ${ }^{1}$ & 1 & 60.54 & 24.75 & 0.23 & 0.006 & 4.72 & 8.79 & 0.80 & 0.17 & 100.0 \\
\hline MS-12-33.1_xlC5 & 3 & 62.23 & 24.64 & 0.23 & 0.018 & 5.43 & 8.11 & 0.51 & 0.10 & 101.3 \\
\hline MS-15-12.5_xlJ4a & 2 & 61.50 & 23.85 & 0.24 & 0.000 & 5.52 & 7.70 & 0.63 & 0.12 & 99.6 \\
\hline MS-15-12.5_xlJ4b & 2 & 62.59 & 24.12 & 0.23 & 0.000 & 5.24 & 8.00 & 0.67 & 0.15 & 101.0 \\
\hline MS-15-12.4_xl2a & 2 & 62.13 & 23.99 & 0.24 & 0.000 & 5.87 & 7.31 & 0.73 & 0.11 & 100.5 \\
\hline MS-15-12.4_x12b & 2 & 62.68 & 23.84 & 0.23 & 0.011 & 5.46 & 7.52 & 0.75 & 0.18 & 99.8 \\
\hline MS-15-12.4_x14b & 2 & 61.50 & 23.85 & 0.24 & 0.000 & 5.52 & 7.70 & 0.63 & 0.12 & 99.6 \\
\hline MS-15-12.4_x14b & 2 & 62.59 & 24.12 & 0.23 & 0.000 & 5.24 & 8.00 & 0.67 & 0.15 & 101.0 \\
\hline $\begin{array}{l}\text { MS-15-12_xl4 } \\
\text { sanidine }\end{array}$ & 2 & 55.84 & 21.86 & 0.23 & 0.000 & 5.56 & 7.38 & 0.62 & N/A & 91.5 \\
\hline MS-11-4.1_xl3a ${ }^{1}$ & 1 & 62.71 & 20.82 & 0.02 & 0.000 & 0.22 & 4.52 & 9.67 & 2.04 & 100.0 \\
\hline MS-11-4.1_xl3b ${ }^{1}$ & 1 & 63.25 & 20.65 & 0.08 & 0.000 & 0.18 & 4.89 & 8.98 & 1.98 & 100.0 \\
\hline MS-10-26_xl3 & 4 & 66.67 & 19.29 & 0.22 & 0.005 & 0.30 & 6.70 & 6.25 & N/A & 99.4 \\
\hline MS-10-26_xl4 & 4 & 66.91 & 19.15 & 0.20 & 0.000 & 0.23 & 6.06 & 7.03 & N/A & 99.6 \\
\hline MS-12-21_xl2a1 & N/A & 77.64 & 14.45 & 0.77 & 0.050 & 0.72 & 2.82 & 3.46 & 0.08 & 100.0 \\
\hline MS-12-21_x12b ${ }^{1}$ & N/A & 77.45 & 14.49 & 0.84 & 0.036 & 0.69 & 3.05 & 3.40 & 0.04 & 100.0 \\
\hline MS-12-33.1_xlC3 & 3 & 67.84 & 19.81 & 0.22 & 0.000 & 0.00 & 7.16 & 6.61 & 0.16 & 101.8 \\
\hline MS-15-18-2T_xl2 & 4 & 66.34 & 19.55 & 0.00 & 0.001 & 0.08 & 3.45 & 11.50 & N/A & 100.9 \\
\hline MS-15-18-2T_xl5 & 4 & 68.57 & 19.07 & 0.19 & 0.005 & 0.17 & 6.66 & 6.63 & N/A & 101.3 \\
\hline MS-15-28.1b_x12a & 2 & 66.10 & 20.11 & 0.16 & 0.001 & 0.62 & 6.06 & 7.01 & 0.21 & 100.3 \\
\hline
\end{tabular}


Table E1 continued.

\begin{tabular}{|c|c|c|c|c|c|c|c|c|c|c|}
\hline $\begin{array}{l}\text { Feldspar type, } \\
\text { sample and } \\
\text { crystal number** }\end{array}$ & $\begin{array}{c}\text { Sample } \\
\text { Unit }\end{array}$ & $\mathrm{SiO}_{2}$ & $\mathrm{Al}_{2} \mathrm{O}_{3}$ & $\mathrm{FeO}^{*}$ & MgO & $\mathrm{CaO}$ & $\mathrm{Na}_{2} \mathrm{O}$ & $\mathbf{K}_{2} \mathbf{O}$ & $\mathbf{B a O}$ & Total \\
\hline \multicolumn{11}{|l|}{ sanidine cont. } \\
\hline MS-15-28.1b_x12b & 2 & 66.31 & 19.97 & 0.17 & 0.001 & 0.89 & 6.30 & 6.56 & 0.23 & 100.4 \\
\hline MS-15-7c_xl1 & 4 & 68.10 & 19.00 & 0.18 & 0.000 & 0.16 & 5.47 & 8.17 & N/A & 101.1 \\
\hline MS-15-7c_xl3 & 4 & 68.30 & 19.31 & 0.17 & 0.000 & 0.22 & 6.29 & 7.15 & N/A & 101.4 \\
\hline MS-15-7c_xl4 & 4 & 68.14 & 19.45 & 0.15 & 0.000 & 0.21 & 5.95 & 7.69 & N/A & 101.6 \\
\hline MS-15-7c_x17 & 4 & 68.57 & 19.31 & 0.21 & 0.000 & 0.28 & 6.58 & 6.44 & N/A & 101.4 \\
\hline
\end{tabular}

** Letters following crystal number indicate multiple analysis points per crystal.

1. Normalized values 
Table E2. Non-unit designating end member composition by feldspar type.

\begin{tabular}{|c|c|c|c|c|}
\hline $\begin{array}{l}\text { Feldspar type, sample and crystal } \\
\text { number** }\end{array}$ & $\begin{array}{c}\text { Sample } \\
\text { Unit }\end{array}$ & $\mathbf{A n}^{2}$ & $\mathbf{A} \mathbf{b}^{3}$ & Or ${ }^{4}$ \\
\hline \multicolumn{5}{|l|}{ Albite } \\
\hline MS-12-33.1 xlC1 & 3 & 7.11 & 83.62 & 9.28 \\
\hline \multicolumn{5}{|l|}{ Andesine } \\
\hline MS-10-20 x11a & 2 & 37.61 & 60.12 & 2.27 \\
\hline MS-10-20 xl1b & 2 & 32.71 & 64.61 & 2.69 \\
\hline MS-14-20a x1a & N/A & 44.46 & 53.50 & 2.04 \\
\hline MS-14-20a xl1b & N/A & 36.38 & 60.87 & 2.75 \\
\hline MS-12-20 x14a & 4 & 37.61 & 60.12 & 2.27 \\
\hline MS-12-20 x14b & 4 & 32.71 & 64.61 & 2.69 \\
\hline MS-15-12 x12 & 2 & 30.30 & 65.69 & 4.01 \\
\hline MS-15-12 x13 & 2 & 39.71 & 57.70 & 2.59 \\
\hline MS-15-12 x15 & 2 & 43.85 & 54.94 & 1.21 \\
\hline MS-15-38 x15 & 1 & 39.60 & 58.57 & 1.83 \\
\hline MS-15-12.4 xl1a & 2 & 37.05 & 59.66 & 3.29 \\
\hline MS-15-12.4 xl1b & 2 & 39.25 & 57.70 & 3.05 \\
\hline MS-15-12.4 xl3a & 2 & 33.93 & 62.39 & 3.68 \\
\hline MS-15-12.4 xl3b & 2 & 30.17 & 65.61 & 4.22 \\
\hline MS-15-12.4 x15a & 2 & 26.94 & 68.35 & 4.72 \\
\hline MS-15-12.4 xl5b & 2 & 36.57 & 60.05 & 3.39 \\
\hline MS-15-12.4 xl6a & 2 & 30.85 & 65.32 & 3.84 \\
\hline MS-15-12.4 xl6b & 2 & 27.95 & 67.51 & 4.54 \\
\hline MS-15-12.4 x18a & 2 & 32.49 & 64.00 & 3.51 \\
\hline MS-15-12.4 xl8b & 2 & 38.28 & 58.63 & 3.10 \\
\hline MS-15-12.4 xl10a & 2 & 41.02 & 56.76 & 2.22 \\
\hline MS-15-12.4 xl10b & 2 & 43.36 & 54.29 & 2.36 \\
\hline MS-15-12.5 x1J2a & 2 & 41.78 & 55.63 & 2.59 \\
\hline MS-15-12.5 xlJ2b & 2 & 42.26 & 55.44 & 2.30 \\
\hline MS-15-12.5 xlJ3a & 2 & 45.19 & 52.84 & 1.98 \\
\hline MS-15-12.5 xlJ3b & 2 & 39.94 & 57.37 & 2.69 \\
\hline MS-15-12.5 x1J5a & 2 & 29.03 & 66.39 & 4.59 \\
\hline MS-15-12.5 xlJ5b & 2 & 33.02 & 63.64 & 3.34 \\
\hline MS-15-12.5 xlJ6a & 2 & 32.94 & 63.13 & 3.93 \\
\hline MS-15-12.5 xlJ6b & 2 & 31.53 & 64.56 & 3.92 \\
\hline MS-15-12.5 xlJ7a & 2 & 42.49 & 55.20 & 2.32 \\
\hline MS-15-12.5 xlJ7b & 2 & 42.23 & 55.51 & 2.26 \\
\hline MS-15-12.5 xlJ10a & 2 & 35.87 & 60.81 & 3.33 \\
\hline MS-15-12.5 xlJ10b & 2 & 36.35 & 60.37 & 3.28 \\
\hline MS-15-12.5 xlK1a & 2 & 36.19 & 61.60 & 2.21 \\
\hline MS-15-12.5 xlK1b & 2 & 38.39 & 59.55 & 2.07 \\
\hline
\end{tabular}


Table E2 continued.

\begin{tabular}{|c|c|c|c|c|}
\hline $\begin{array}{l}\text { Feldspar type, sample and crystal } \\
\text { number** }\end{array}$ & $\begin{array}{c}\text { Sample } \\
\text { Unit }\end{array}$ & $\mathbf{A n}^{2}$ & $\mathbf{A} \mathbf{b}^{3}$ & Or $\mathbf{r}^{4}$ \\
\hline \multicolumn{5}{|l|}{ andesine cont. } \\
\hline MS-14-4b x11a & N/A & 44.90 & 52.60 & 2.50 \\
\hline MS-14-4b xl1b & N/A & 43.19 & 54.34 & 2.47 \\
\hline MS-15-28.1b xl3a & 2 & 48.41 & 49.57 & 2.02 \\
\hline MS-15-28.1b xl3b & 2 & 45.49 & 52.18 & 2.32 \\
\hline MS-15-28.1b xl6a & 2 & 40.31 & 57.42 & 2.27 \\
\hline MS-15-28.1b xl6b & 2 & 41.16 & 56.32 & 2.53 \\
\hline \multicolumn{5}{|l|}{ anorthoclase } \\
\hline MS-10-26 xl5 & 4 & 1.96 & 65.14 & 32.93 \\
\hline MS-12-33.1 xlC4 & 3 & 1.06 & 71.96 & 27.00 \\
\hline MS-12-33.1 xlH7a & 3 & 1.18 & 68.21 & 30.63 \\
\hline MS-12-33.1 xlH7b & 3 & 1.07 & 68.13 & 30.83 \\
\hline MS-15-28.1b x14a & 2 & 2.06 & 68.07 & 29.90 \\
\hline MS-15-28.1b xl4b & 2 & 2.77 & 69.17 & 28.09 \\
\hline \multicolumn{5}{|l|}{ bytownite } \\
\hline MS-14-20a xl2a & N/A & 74.86 & 24.20 & 0.93 \\
\hline MS-14-20a x12b & N/A & 65.90 & 33.03 & 1.07 \\
\hline MS-14-20a xl3a & N/A & 83.36 & 16.26 & 0.38 \\
\hline MS-14-20a xl3b & N/A & 77.18 & 22.32 & 0.49 \\
\hline MS-14-20a x15a & N/A & 74.11 & 25.20 & 0.68 \\
\hline MS-14-20a xl5b & N/A & 67.25 & 31.49 & 1.26 \\
\hline MS-15-18-1T xl2a & N/A & 74.70 & 24.75 & 0.55 \\
\hline MS-15-18-1T xl2b & N/A & 73.64 & 25.77 & 0.58 \\
\hline MS-15-18-1T xl4a & N/A & 72.81 & 26.71 & 0.48 \\
\hline MS-15-18-1T xl4b & N/A & 69.64 & 29.63 & 0.73 \\
\hline MS-15-18-1T xl5a & N/A & 57.27 & 41.50 & 1.23 \\
\hline MS-15-18-1T xl5b & N/A & 97.22 & 2.76 & 0.02 \\
\hline MS-15-18-1T xl6a & N/A & 72.01 & 27.48 & 0.51 \\
\hline MS-15-18-1T xl6b & N/A & 68.22 & 31.14 & 0.64 \\
\hline MS-15-12.3 x17a & 2 & 72.71 & 26.79 & 0.51 \\
\hline MS-15-12.3 x17b & 2 & 72.38 & 27.14 & 0.47 \\
\hline \multicolumn{5}{|l|}{ bytownite } \\
\hline MS-15-18.2 xl8a & 2 & 70.67 & 28.69 & 0.64 \\
\hline MS-15-18.2 x18b & 2 & 71.82 & 27.63 & 0.54 \\
\hline MS-15-18.2 x19a & 2 & 72.22 & 27.21 & 0.56 \\
\hline MS-15-18.2 x19b & 2 & 71.21 & 28.37 & 0.42 \\
\hline \multicolumn{5}{|l|}{ labradorite } \\
\hline MS-10-26 x11 & 4 & 56.05 & 42.67 & 1.28 \\
\hline MS-14-20a xl4a & N/A & 72.86 & 26.44 & 0.70 \\
\hline
\end{tabular}


Table E2 continued.

\begin{tabular}{|c|c|c|c|c|}
\hline $\begin{array}{l}\text { Feldspar type, sample and crystal } \\
\text { number** }\end{array}$ & $\begin{array}{c}\text { Sample } \\
\text { Unit }\end{array}$ & $\mathbf{A n}^{2}$ & $\mathbf{A} \mathbf{b}^{3}$ & $\mathrm{Or}^{4}$ \\
\hline \multicolumn{5}{|l|}{ labradorite cont. } \\
\hline MS-14-20a xl4b & N/A & 64.44 & 34.35 & 1.21 \\
\hline MS-14-20a x16a & N/A & 61.06 & 37.94 & 0.99 \\
\hline MS-14-20a xl6b & N/A & 64.78 & 34.26 & 0.96 \\
\hline MS-15-12.3 xl1a & 2 & 58.15 & 40.76 & 1.09 \\
\hline MS-15-12.3 x11b & 2 & 69.34 & 29.81 & 0.85 \\
\hline MS-15-18.2 x13a & 2 & 67.06 & 32.25 & 0.70 \\
\hline MS-15-18.2 x13b & 2 & 69.02 & 30.33 & 0.65 \\
\hline MS-15-12.5 xlK3a & 2 & 50.32 & 48.15 & 1.53 \\
\hline MS-15-12.5 xlK3b & 2 & 51.65 & 46.72 & 1.63 \\
\hline MS-15-18-2T xl1 & 4 & 55.58 & 43.11 & 1.31 \\
\hline MS-15-18-1T xl3a & $\mathrm{N} / \mathrm{A}$ & 52.57 & 46.13 & 1.31 \\
\hline MS-15-18-1T xl3b & N/A & 50.96 & 47.61 & 1.43 \\
\hline MS-15-28.1b x17a & 2 & 55.88 & 42.98 & 1.14 \\
\hline MS-15-28.1b xl7b & 2 & 53.04 & 45.37 & 1.59 \\
\hline \multicolumn{5}{|l|}{ oligoclase } \\
\hline MS-11-4.1 x17a $\mathrm{a}^{1}$ & 1 & 21.68 & 73.92 & 4.41 \\
\hline MS-11-4.1 x17b ${ }^{1}$ & 1 & 21.92 & 73.66 & 4.43 \\
\hline MS-12-33.1 xlC5 & 3 & 26.31 & 70.73 & 2.97 \\
\hline MS-15-12.5 xlJ4a & 2 & 27.41 & 68.89 & 3.70 \\
\hline MS-15-12.5 xlJ4b & 2 & 25.62 & 70.50 & 3.87 \\
\hline MS-15-12.4 xl2a & 2 & 29.48 & 66.16 & 4.37 \\
\hline MS-15-12.4 x12b & 2 & 27.41 & 68.13 & 4.47 \\
\hline MS-15-12 x14 & 2 & 28.36 & 67.85 & 3.79 \\
\hline \multicolumn{5}{|l|}{ sanidine } \\
\hline MS-11-4.1 xl3a ${ }^{1}$ & 1 & 1.09 & 40.97 & 57.97 \\
\hline MS-11-4.1 xl3b & 1 & 0.90 & 44.77 & 54.36 \\
\hline MS-10-26 x13 & 4 & 1.53 & 60.92 & 37.57 \\
\hline MS-10-26 x14 & 4 & 1.19 & 55.92 & 42.93 \\
\hline MS-12-21 xl2a $\mathrm{a}^{1}$ & $\mathrm{~N} / \mathrm{A}$ & 7.23 & 51.27 & 41.52 \\
\hline MS-12-21 x12b & N/A & 6.71 & 53.75 & 39.56 \\
\hline MS-12-33.1 xlC3 & 3 & 0.00 & 62.14 & 37.89 \\
\hline MS-15-18-2T x12 & 4 & 0.41 & 31.08 & 68.54 \\
\hline MS-15-18-2T xl5 & 4 & 0.87 & 59.83 & 39.33 \\
\hline MS-15-28.1b xl2a & 2 & 3.11 & 54.90 & 42.02 \\
\hline MS-15-28.1b xl2b & 2 & 4.45 & 56.59 & 38.99 \\
\hline MS-15-7c xl1 & 4 & 0.79 & 49.95 & 49.30 \\
\hline MS-15-7c xl3 & 4 & 1.09 & 56.47 & 42.47 \\
\hline MS-15-7c xl4 & 4 & 1.02 & 53.38 & 45.63 \\
\hline
\end{tabular}


Table E2 continued.

\begin{tabular}{|c|c|c|c|c|}
\hline $\begin{array}{l}\text { Feldspar type, sample and crystal } \\
\text { number** }\end{array}$ & $\begin{array}{l}\text { Sample } \\
\text { Unit }\end{array}$ & $\mathbf{A n}^{2}$ & $\mathbf{A} \mathbf{b}^{\mathbf{3}}$ & $O r^{4}$ \\
\hline \multicolumn{5}{|l|}{ sanidine cont. } \\
\hline MS-15-7c x17 & 4 & 1.42 & 59.86 & 38.75 \\
\hline $\begin{array}{l}\text { ** Letters following crystal number } \\
\text { 1. Normalized values. } \\
\text { 2. } \mathrm{An}=(\mathrm{CaO} * 0.714 / 40) /(\mathrm{CaO} * 0.71 \\
\text { 3. } \mathrm{Ab}=\left(\mathrm{Na}_{2} \mathrm{O} * 0.74 / 23\right) /(\mathrm{CaO} * 0.71 \\
\text { 4. } \mathrm{Or}=\left(\mathrm{K}_{2} \mathrm{O} * 0.83 / 39\right) /(\mathrm{CaO} * 0.714\end{array}$ & $\begin{array}{l}\text { Oultiple an } \\
\mathrm{O} * 0.74 / 23 \\
\mathrm{O} 0.74 / 23 \\
* 0.74 / 23+\end{array}$ & $\begin{array}{l}\text { ints pe } \\
0.83 / 39 \\
.829 / 3 \\
83 / 39)^{\prime}\end{array}$ & $\begin{array}{l}\text { stal. } \\
0 \\
0\end{array}$ & \\
\hline
\end{tabular}




\section{APPENDIX F：BULK ROCK CHEMICAL DATA}

Table F1. Unit 1 bulk tuff major and trace element geochemical data from Streck et al., (2015).

\begin{tabular}{|c|c|c|c|c|c|c|c|c|}
\hline Sample & $\begin{array}{c}\text { CR- } \\
\text { U20b }\end{array}$ & $\begin{array}{l}\text { CR- } \\
\text { U21 }\end{array}$ & $\begin{array}{l}\text { CR- } \\
\text { U22b }\end{array}$ & $\begin{array}{l}\text { CR- } \\
\text { U22d }\end{array}$ & $\begin{array}{c}\text { MS-DIT } \\
1\end{array}$ & $\begin{array}{c}\text { MS- } \\
\text { NEMT }\end{array}$ & $\begin{array}{c}\text { MS- } \\
\text { MC1-09 }\end{array}$ & $\begin{array}{c}\text { MS- } \\
\text { MC2-09 }\end{array}$ \\
\hline \multicolumn{9}{|c|}{ XRF normalized (weight \%) } \\
\hline $\mathrm{SiO}_{2}$ & 78.1 & 76.1 & 76.2 & 75.8 & 75.8 & 75.9 & 75.7 & 76.1 \\
\hline $\mathrm{TiO}_{2}$ & 0.2 & 0.2 & 0.2 & 0.2 & 0.2 & 0.2 & 0.2 & 0.2 \\
\hline $\mathrm{Al}_{2} \mathrm{O}_{3}$ & 12.5 & 13.0 & 12.4 & 12.7 & 12.3 & 12.6 & 12.3 & 12.3 \\
\hline $\mathrm{FeO}^{*}$ & 0.4 & 1.7 & 2.0 & 2.2 & 2.1 & 1.9 & 1.9 & 2.1 \\
\hline $\mathrm{MnO}$ & 0.0 & 0.0 & 0.1 & 0.1 & 0.0 & 0.0 & 0.0 & 0.0 \\
\hline $\mathrm{MgO}$ & 0.0 & 0.1 & 0.0 & 0.2 & 0.1 & 0.1 & 0.2 & 0.1 \\
\hline $\mathrm{CaO}$ & 0.2 & 0.6 & 0.6 & 0.7 & 0.6 & 0.6 & 1.0 & 0.8 \\
\hline $\mathrm{Na}_{2} \mathrm{O}$ & 4.7 & 4.5 & 3.7 & 3.1 & 2.7 & 2.5 & 3.6 & 2.7 \\
\hline $\mathrm{K}_{2} \mathrm{O}$ & 3.9 & 3.8 & 4.9 & 5.2 & 6.2 & 6.2 & 5.1 & 5.5 \\
\hline $\mathrm{P}_{2} \mathrm{O}_{5}$ & 0.0 & 0.0 & 0.0 & 0.0 & 0.0 & 0.0 & 0.0 & 0.1 \\
\hline Total & 100.0 & 100.0 & 100.0 & 100.0 & 100.0 & 100.0 & 100.0 & 100.0 \\
\hline \multicolumn{9}{|c|}{ XRF unnormalized (ppm) } \\
\hline $\mathrm{Ni}$ & 0.0 & 2.0 & 0.0 & 0.2 & 0.0 & 0.0 & 2.2 & 4.5 \\
\hline $\mathrm{Cr}$ & 2.0 & 4.5 & 1.4 & 3.0 & 1.9 & 2.5 & 2.9 & 2.7 \\
\hline $\mathrm{Sc}$ & 3.5 & 4.9 & 3.8 & 5.1 & 3.6 & 3.7 & 4.3 & 4.1 \\
\hline V & 1.1 & 15.4 & 3.7 & 8.3 & 1.4 & 3.3 & 4.7 & 7.6 \\
\hline $\mathrm{Ba}$ & 1487.5 & 1405.6 & 1400.3 & 1366.5 & 1349.8 & 1350.8 & 1318.0 & 1325.5 \\
\hline $\mathrm{Rb}$ & 75.5 & 73.1 & 74.3 & 71.4 & 78.4 & 87.3 & 70.6 & 73.7 \\
\hline $\mathrm{Sr}$ & 29.1 & 43.6 & 25.5 & 31.0 & 25.6 & 29.2 & 71.7 & 39.8 \\
\hline $\mathrm{Zr}$ & 431.0 & 416.1 & 412.0 & 416.4 & 405.5 & 410.5 & 417.6 & 422.3 \\
\hline $\mathrm{Y}$ & 125.6 & 91.4 & 90.3 & 85.8 & 94.2 & 87.6 & 90.5 & 93.9 \\
\hline $\mathrm{Nb}$ & 24.8 & 22.7 & 23.1 & 23.3 & 23.6 & 22.8 & 22.2 & 24.1 \\
\hline $\mathrm{Ga}$ & 20.8 & 20.6 & 20.2 & 20.7 & 21.2 & 20.9 & 20.4 & 21.3 \\
\hline $\mathrm{Cu}$ & 0.0 & 4.6 & 0.5 & 1.3 & 2.3 & 0.7 & 3.7 & 6.1 \\
\hline $\mathrm{Zn}$ & 65.4 & 209.1 & 149.7 & 152.8 & 169.4 & 141.6 & 145.1 & 139.5 \\
\hline $\mathrm{Pb}$ & 14.5 & 16.3 & 16.1 & 17.6 & 17.1 & 16.7 & 17.4 & 18.1 \\
\hline $\mathrm{La}$ & 67.4 & 51.3 & 45.3 & 43.3 & 47.4 & 43.5 & 45.1 & 44.7 \\
\hline $\mathrm{Ce}$ & 132.1 & 103.9 & 95.0 & 89.6 & 101.2 & 91.6 & 93.2 & 97.1 \\
\hline Th & 11.3 & 9.2 & 9.2 & 10.0 & 9.6 & 9.0 & 7.8 & 6.9 \\
\hline $\mathrm{Nd}$ & 65.6 & 52.2 & 45.8 & 44.3 & 50.8 & 48.0 & 46.6 & 48.8 \\
\hline $\mathrm{U}$ & 2.7 & 2.6 & 2.6 & 3.3 & 3.3 & 3.5 & 2.9 & 2.3 \\
\hline
\end{tabular}


Table F1 continued.

\begin{tabular}{|c|c|c|c|c|c|c|c|c|}
\hline Sample & $\begin{array}{l}\text { CR- } \\
\text { U20b }\end{array}$ & $\begin{array}{l}\text { CR- } \\
\text { U21 }\end{array}$ & $\begin{array}{l}\text { CR- } \\
\text { U22b }\end{array}$ & $\begin{array}{c}\text { CR- } \\
\text { U22d } \\
\end{array}$ & $\begin{array}{c}\text { MS-DIT } \\
1 \\
\end{array}$ & $\begin{array}{c}\text { MS- } \\
\text { NEMT } \\
\end{array}$ & $\begin{array}{c}\text { MS- } \\
\text { MC1-09 } \\
\end{array}$ & $\begin{array}{c}\text { MS- } \\
\text { MC2-09 } \\
\end{array}$ \\
\hline \multicolumn{9}{|c|}{ ICP-MS (ppm) } \\
\hline $\mathrm{La}$ & 69.8 & 51.7 & 45.8 & 43.8 & 49.1 & 44.7 & 44.6 & 46.2 \\
\hline $\mathrm{Ce}$ & 137.8 & 106.2 & 96.7 & 93.1 & 102.1 & 94.6 & 93.5 & 96.1 \\
\hline $\operatorname{Pr}$ & 17.1 & 13.7 & 12.3 & 11.8 & 13.2 & 12.0 & 11.8 & 12.3 \\
\hline $\mathrm{Nd}$ & 69.5 & 54.9 & 48.9 & 47.1 & 52.2 & 48.2 & 47.4 & 49.2 \\
\hline Sm & 15.8 & 12.7 & 11.5 & 11.2 & 12.2 & 11.2 & 11.1 & 11.5 \\
\hline $\mathrm{Eu}$ & 2.1 & 2.0 & 1.7 & 1.6 & 1.6 & 1.6 & 1.6 & 1.7 \\
\hline Gd & 16.8 & 13.3 & 12.0 & 11.7 & 12.9 & 11.6 & 11.4 & 12.1 \\
\hline $\mathrm{Tb}$ & 3.0 & 2.4 & 2.3 & 2.2 & 2.4 & 2.2 & 2.2 & 2.3 \\
\hline Dy & 20.0 & 16.2 & 15.3 & 14.8 & 16.3 & 14.9 & 14.8 & 15.5 \\
\hline Ho & 4.4 & 3.5 & 3.4 & 3.3 & 3.6 & 3.3 & 3.2 & 3.4 \\
\hline $\mathrm{Er}$ & 12.7 & 10.1 & 10.1 & 9.6 & 10.5 & 9.7 & 9.7 & 10.2 \\
\hline $\mathrm{Tm}$ & 1.9 & 1.6 & 1.6 & 1.5 & 1.6 & 1.5 & 1.5 & 1.6 \\
\hline $\mathrm{Yb}$ & 12.0 & 10.0 & 10.3 & 9.9 & 10.7 & 10.0 & 9.9 & 10.2 \\
\hline $\mathrm{Lu}$ & 2.0 & 1.6 & 1.6 & 1.6 & 1.7 & 1.6 & 1.6 & 1.7 \\
\hline $\mathrm{Ba}$ & 1528.0 & 1443.1 & 1429.5 & 1398.3 & 1362.5 & 1365.9 & 1347.2 & 1346.6 \\
\hline Th & 8.2 & 7.8 & 7.8 & 7.8 & 7.7 & 7.6 & 7.6 & 7.7 \\
\hline $\mathrm{Nb}$ & 24.5 & 23.1 & 23.1 & 23.0 & 22.8 & 22.7 & 22.7 & 23.8 \\
\hline Y & 125.3 & 89.9 & 89.1 & 86.1 & 93.0 & 86.2 & 87.4 & 90.5 \\
\hline $\mathrm{Hf}$ & 12.0 & 11.5 & 11.6 & 11.7 & 11.4 & 11.4 & 11.3 & 11.5 \\
\hline $\mathrm{Ta}$ & 1.5 & 1.4 & 1.4 & 1.4 & 1.4 & 1.4 & 1.4 & 1.4 \\
\hline $\mathrm{U}$ & 3.9 & 3.5 & 3.5 & 3.3 & 3.4 & 3.4 & 3.3 & 3.4 \\
\hline $\mathrm{Pb}$ & 15.8 & 16.2 & 17.3 & 17.1 & 17.1 & 16.7 & 16.4 & 16.7 \\
\hline $\mathrm{Rb}$ & 76.3 & 73.0 & 73.8 & 72.2 & 77.7 & 86.8 & 70.0 & 73.2 \\
\hline Cs & 1.2 & 2.9 & 2.9 & 2.8 & 2.9 & 3.0 & 2.7 & 2.8 \\
\hline $\mathrm{Sr}$ & 29.9 & 43.7 & 26.7 & 31.7 & 25.9 & 31.9 & 72.5 & 40.8 \\
\hline $\mathrm{Sc}$ & 3.6 & 4.9 & 4.0 & 4.7 & 3.8 & 4.2 & 4.4 & 4.7 \\
\hline $\mathrm{Zr}$ & 459.7 & 438.5 & 434.0 & 436.1 & 419.8 & 427.5 & 419.6 & 432.3 \\
\hline
\end{tabular}


Table F1 continued.

\begin{tabular}{|c|c|c|c|c|c|c|c|}
\hline Sample & MS-10-23 & MS-11-3 & MS-11-27 & MS-11-27 & $\begin{array}{c}\text { MS-11-54 } \\
\text { lit }\end{array}$ & $\begin{array}{c}\text { MS-11-54 } \\
\text { drk }\end{array}$ & $\begin{array}{c}\text { MS-12- } \\
29.1\end{array}$ \\
\hline \multicolumn{8}{|c|}{ XRF normalized (weight \%) } \\
\hline $\mathrm{SiO}_{2}$ & 76.6 & 75.7 & 73.1 & 73.0 & 75.5 & 75.6 & 76.3 \\
\hline $\mathrm{TiO}_{2}$ & 0.2 & 0.2 & 0.2 & 0.2 & 0.2 & 0.2 & 0.2 \\
\hline $\mathrm{Al}_{2} \mathrm{O}_{3}$ & 12.4 & 12.8 & 16.0 & 16.1 & 13.6 & 13.6 & 12.3 \\
\hline $\mathrm{FeO} *$ & 2.0 & 2.8 & 2.7 & 2.7 & 2.5 & 2.3 & 2.0 \\
\hline $\mathrm{MnO}$ & 0.1 & 0.1 & 0.1 & 0.1 & 0.1 & 0.1 & 0.1 \\
\hline $\mathrm{MgO}$ & 0.1 & 0.1 & 0.3 & 0.2 & 0.0 & 0.1 & 0.0 \\
\hline $\mathrm{CaO}$ & 0.6 & 0.6 & 0.7 & 0.7 & 0.7 & 0.5 & 0.6 \\
\hline $\mathrm{Na}_{2} \mathrm{O}$ & 3.4 & 3.2 & 3.3 & 3.4 & 3.3 & 3.0 & 3.8 \\
\hline $\mathrm{K}_{2} \mathrm{O}$ & 4.6 & 4.5 & 3.7 & 3.7 & 4.2 & 4.6 & 4.7 \\
\hline $\mathrm{P}_{2} \mathrm{O}_{5}$ & 0.0 & 0.0 & 0.0 & 0.0 & 0.0 & 0.0 & 0.0 \\
\hline Total & 100.0 & 100.0 & 100.0 & 100.0 & 100.0 & 100.0 & 100.0 \\
\hline \multicolumn{8}{|c|}{ XRF unnormalized (ppm) } \\
\hline $\mathrm{Ni}$ & 1.1 & 3.5 & 1.3 & 4.6 & 3.8 & 0.5 & 2.4 \\
\hline $\mathrm{Cr}$ & 3.4 & 4.8 & 4.1 & 2.8 & 2.8 & 5.3 & 3.4 \\
\hline $\mathrm{Sc}$ & 3.7 & 3.4 & 4.2 & 5.0 & 3.8 & 4.5 & 4.1 \\
\hline V & 4.7 & 7.2 & 8.2 & 11.7 & 4.8 & 7.4 & 3.1 \\
\hline $\mathrm{Ba}$ & 1374.0 & 1315.7 & 1164.0 & 1262.7 & 1331.8 & 1324.6 & 1411.0 \\
\hline $\mathrm{Rb}$ & 74.9 & 75.4 & 57.7 & 59.3 & 69.6 & 78.2 & 74.2 \\
\hline $\mathrm{Sr}$ & 29.5 & 30.9 & 30.5 & 32.6 & 27.8 & 28.9 & 27.6 \\
\hline $\mathrm{Zr}$ & 427.0 & 424.2 & 531.4 & 548.0 & 463.8 & 459.2 & 428.8 \\
\hline $\mathrm{Y}$ & 91.7 & 90.6 & 93.6 & 95.0 & 86.2 & 84.8 & 93.0 \\
\hline $\mathrm{Nb}$ & 23.9 & 22.8 & 28.1 & 28.8 & 26.2 & 25.2 & 24.2 \\
\hline $\mathrm{Ga}$ & 20.6 & 21.7 & 26.0 & 27.1 & 22.3 & 21.7 & 21.5 \\
\hline $\mathrm{Cu}$ & 2.7 & 4.3 & 5.6 & 6.7 & 2.0 & 3.5 & 1.4 \\
\hline $\mathrm{Zn}$ & 148.7 & 151.1 & 152.5 & 157.3 & 166.1 & 152.7 & 152.4 \\
\hline $\mathrm{Pb}$ & 19.4 & 18.7 & 22.9 & 24.7 & 19.7 & 18.2 & 18.7 \\
\hline $\mathrm{La}$ & 43.6 & 45.1 & 48.0 & 50.3 & 42.3 & 40.3 & 47.4 \\
\hline $\mathrm{Ce}$ & 91.3 & 83.8 & 117.9 & 122.0 & 95.9 & 95.6 & 91.9 \\
\hline $\mathrm{Th}$ & 6.6 & 7.9 & 9.6 & 9.6 & 9.6 & 7.1 & 7.6 \\
\hline $\mathrm{Nd}$ & 46.4 & 49.4 & 53.2 & 53.5 & 47.3 & 46.5 & 49.5 \\
\hline $\mathrm{U}$ & 3.3 & 2.9 & 3.7 & 2.5 & 3.5 & 1.9 & 4.7 \\
\hline
\end{tabular}


Table F1 continued.

\begin{tabular}{|c|c|c|c|c|c|c|c|}
\hline Sample & MS-10-23 & MS-11-3 & MS-11-27 & MS-11-27 & $\begin{array}{c}\text { MS-11-54 } \\
\text { lit }\end{array}$ & $\begin{array}{c}\text { MS-11-54 } \\
\text { drk }\end{array}$ & $\begin{array}{c}\text { MS-12- } \\
29.1 \\
\end{array}$ \\
\hline \multicolumn{8}{|c|}{ ICP-MS (ppm) } \\
\hline $\mathrm{La}$ & 47.4 & 48.7 & 51.0 & 51.0 & 44.6 & 44.0 & 46.7 \\
\hline $\mathrm{Ce}$ & 99.6 & 90.8 & 126.8 & 122.1 & 100.1 & 95.5 & 93.3 \\
\hline $\operatorname{Pr}$ & 12.6 & 12.7 & 13.7 & 13.7 & 12.1 & 12.0 & 12.4 \\
\hline $\mathrm{Nd}$ & 50.1 & 50.6 & 55.2 & 54.6 & 47.8 & 47.4 & 49.5 \\
\hline $\mathrm{Sm}$ & 11.7 & 11.8 & 12.8 & 12.8 & 11.3 & 11.2 & 11.6 \\
\hline $\mathrm{Eu}$ & 1.7 & 1.8 & 1.9 & 1.9 & 1.9 & 1.7 & 1.7 \\
\hline $\mathrm{Gd}$ & 12.1 & 12.4 & 13.2 & 13.0 & 11.6 & 11.4 & 12.3 \\
\hline $\mathrm{Tb}$ & 2.3 & 2.3 & 2.4 & 2.4 & 2.2 & 2.2 & 2.3 \\
\hline Dy & 15.4 & 15.4 & 16.2 & 16.3 & 14.8 & 14.5 & 15.6 \\
\hline Ho & 3.4 & 3.4 & 3.6 & 3.5 & 3.2 & 3.2 & 3.4 \\
\hline $\mathrm{Er}$ & 10.0 & 9.8 & 10.4 & 10.4 & 9.5 & 9.6 & 10.1 \\
\hline $\mathrm{Tm}$ & 1.6 & 1.5 & 1.6 & 1.6 & 1.5 & 1.5 & 1.6 \\
\hline $\mathrm{Yb}$ & 10.3 & 9.8 & 10.7 & 10.8 & 9.7 & 9.7 & 10.2 \\
\hline $\mathrm{Lu}$ & 1.7 & 1.6 & 1.8 & 1.8 & 1.6 & 1.5 & 1.6 \\
\hline $\mathrm{Ba}$ & 1470.8 & 1340.8 & 1179.8 & 1276.6 & 1362.4 & 1359.4 & 1419.1 \\
\hline $\mathrm{Th}$ & 7.8 & 7.7 & 9.7 & 10.0 & 8.4 & 8.2 & 7.8 \\
\hline $\mathrm{Nb}$ & 24.2 & 20.5 & 27.3 & 28.0 & 25.1 & 24.6 & 23.3 \\
\hline $\mathrm{Y}$ & 90.3 & 89.8 & 92.0 & 90.7 & 83.6 & 83.5 & 90.3 \\
\hline $\mathrm{Hf}$ & 11.8 & 11.4 & 14.2 & 14.9 & 12.4 & 12.4 & 11.6 \\
\hline $\mathrm{Ta}$ & 1.4 & 1.2 & 1.7 & 1.8 & 1.5 & 1.5 & 1.4 \\
\hline $\mathrm{U}$ & 3.5 & 3.5 & 3.1 & 3.2 & 3.7 & 3.5 & 3.5 \\
\hline $\mathrm{Pb}$ & 17.4 & 17.5 & 21.6 & 22.1 & 18.4 & 16.6 & 17.5 \\
\hline $\mathrm{Rb}$ & 77.2 & 74.8 & 57.0 & 58.1 & 68.4 & 78.5 & 74.6 \\
\hline Cs & 3.0 & 2.9 & 2.5 & 2.6 & 3.1 & 3.0 & 2.9 \\
\hline $\mathrm{Sr}$ & 30.3 & 32.7 & 31.8 & 34.5 & 28.7 & 30.6 & 30.9 \\
\hline $\mathrm{Sc}$ & 5.6 & 4.3 & 4.2 & 4.3 & 4.1 & 4.4 & 3.8 \\
\hline $\mathrm{Zr}$ & 461.3 & 450.8 & 557.0 & 551.5 & 491.5 & 490.6 & 438.9 \\
\hline
\end{tabular}


Table F1 continued.

\begin{tabular}{|c|c|c|c|c|c|c|}
\hline Sample & $\begin{array}{c}\text { STRM MS- } \\
12-26\end{array}$ & MS15-32* & MS15-34A* & MS15-38* & PFC1548* & $\begin{array}{l}\text { CR- } \\
\text { U31c }\end{array}$ \\
\hline \multicolumn{7}{|c|}{ XRF normalized (weight \%) } \\
\hline $\mathrm{SiO}_{2}$ & 76.2 & 76.1 & 75.7 & 75.5 & 75.9 & 77.2 \\
\hline $\mathrm{TiO}_{2}$ & 0.2 & 0.2 & 0.2 & 0.2 & 0.2 & 0.2 \\
\hline $\mathrm{Al}_{2} \mathrm{O}_{3}$ & 12.4 & 12.3 & 12.4 & 12.7 & 12.3 & 12.8 \\
\hline $\mathrm{FeO}^{*}$ & 1.9 & 2.2 & 2.4 & 2.4 & 2.1 & 1.2 \\
\hline $\mathrm{MnO}$ & 0.1 & 0.1 & 0.0 & 0.1 & 0.1 & 0.0 \\
\hline $\mathrm{MgO}$ & 0.1 & 0.1 & 0.3 & 0.2 & 0.0 & 0.1 \\
\hline $\mathrm{CaO}$ & 0.6 & 0.5 & 0.6 & 0.7 & 0.5 & 0.4 \\
\hline $\mathrm{Na}_{2} \mathrm{O}$ & 3.3 & 3.1 & 2.1 & 2.5 & 2.8 & 4.2 \\
\hline $\mathrm{K}_{2} \mathrm{O}$ & 5.2 & 5.5 & 6.4 & 5.9 & 6.0 & 3.8 \\
\hline $\mathrm{P}_{2} \mathrm{O}_{5}$ & 0.0 & 0.0 & 0.0 & 0.0 & 0.0 & 0.0 \\
\hline Total & 100.0 & 95.3 & 95.1 & 94.6 & 95.8 & 100.0 \\
\hline \multicolumn{7}{|c|}{ XRF unnormalized (ppm) } \\
\hline $\mathrm{Ni}$ & 2.1 & 3.0 & 2.4 & 1.9 & 1.1 & 2.2 \\
\hline $\mathrm{Cr}$ & 2.6 & 4.0 & 1.8 & 0.8 & 3.7 & 4.5 \\
\hline Sc & 4.3 & 3.3 & 4.9 & 4.3 & 4.3 & 3.2 \\
\hline $\mathrm{V}$ & 7.3 & 6.8 & 4.2 & 5.9 & 4.6 & 13.5 \\
\hline $\mathrm{Ba}$ & 1412.4 & 1365.2 & 1326.7 & 1372.2 & 1445.1 & 1405.8 \\
\hline $\mathrm{Rb}$ & 74.5 & 77.1 & 89.4 & 90.7 & 81.8 & 72.8 \\
\hline $\mathrm{Sr}$ & 33.1 & 29.5 & 33.1 & 35.3 & 28.9 & 37.3 \\
\hline $\mathrm{Zr}$ & 429.8 & 424.9 & 431.4 & 448.7 & 441.8 & 436.4 \\
\hline $\mathrm{Y}$ & 93.7 & 114.3 & 94.3 & 106.3 & 92.2 & 94.6 \\
\hline $\mathrm{Nb}$ & 24.2 & 23.6 & 24.2 & 24.4 & 23.9 & 24.5 \\
\hline $\mathrm{Ga}$ & 22.0 & 20.8 & 20.6 & 21.1 & 20.7 & 21.5 \\
\hline $\mathrm{Cu}$ & 2.4 & 2.9 & 3.8 & 4.4 & 2.6 & 3.1 \\
\hline $\mathrm{Zn}$ & 156.4 & 154.4 & 147.7 & 168.8 & 150.6 & 111.7 \\
\hline $\mathrm{Pb}$ & 19.1 & 17.7 & 17.3 & 18.1 & 19.7 & 16.4 \\
\hline $\mathrm{La}$ & 48.5 & 63.2 & 47.8 & 56.2 & 48.1 & 42.9 \\
\hline $\mathrm{Ce}$ & 97.3 & 98.6 & 95.8 & 99.4 & 92.5 & 92.7 \\
\hline $\mathrm{Th}$ & 8.1 & 8.2 & 8.3 & 6.8 & 7.6 & 8.2 \\
\hline $\mathrm{Nd}$ & 49.6 & 63.8 & 51.8 & 59.5 & 47.8 & 46.2 \\
\hline $\mathrm{U}$ & 4.4 & 2.9 & 4.0 & 4.1 & 3.5 & 3.2 \\
\hline
\end{tabular}


Table F1 continued.

\begin{tabular}{|c|c|c|c|c|c|c|}
\hline Sample & MS-12-26 & MS15-32 & MS15-34A & MS15-38 & PFC1548 & $\begin{array}{l}\text { CR- } \\
\text { U31c }\end{array}$ \\
\hline \multicolumn{7}{|c|}{ ICP-MS (ppm) } \\
\hline $\mathrm{La}$ & 47.1 & 64.8 & 50.6 & 61.5 & 46.6 & 43.7 \\
\hline $\mathrm{Ce}$ & 98.6 & 105.7 & 97.6 & 102.7 & 95.7 & 91.7 \\
\hline $\operatorname{Pr}$ & 12.5 & 17.3 & 13.3 & 15.7 & 12.4 & 11.5 \\
\hline $\mathrm{Nd}$ & 50.4 & 69.2 & 53.2 & 62.5 & 49.7 & 45.5 \\
\hline $\mathrm{Sm}$ & 11.6 & 16.1 & 12.3 & 14.4 & 11.7 & 10.8 \\
\hline $\mathrm{Eu}$ & 1.7 & 2.2 & 1.7 & 2.2 & 1.7 & 1.8 \\
\hline $\mathrm{Gd}$ & 12.3 & 16.5 & 12.6 & 15.2 & 12.3 & 11.3 \\
\hline $\mathrm{Tb}$ & 2.3 & 3.1 & 2.4 & 2.8 & 2.3 & 2.2 \\
\hline Dy & 15.6 & 20.4 & 16.0 & 18.6 & 15.5 & 15.0 \\
\hline Ho & 3.4 & 4.4 & 3.5 & 4.0 & 3.4 & 3.4 \\
\hline $\mathrm{Er}$ & 10.0 & 12.7 & 10.2 & 11.8 & 10.2 & 10.0 \\
\hline $\mathrm{Tm}$ & 1.6 & 2.0 & 1.6 & 1.8 & 1.6 & 1.6 \\
\hline $\mathrm{Yb}$ & 10.3 & 12.7 & 10.4 & 11.7 & 10.5 & 10.3 \\
\hline $\mathrm{Lu}$ & 1.7 & 2.1 & 1.7 & 1.9 & 1.7 & 1.7 \\
\hline $\mathrm{Ba}$ & 1418.9 & 1414.2 & 1381.4 & 1425.9 & 1474.4 & 1423.4 \\
\hline Th & 7.9 & 8.0 & 8.1 & 8.1 & 7.8 & 8.1 \\
\hline $\mathrm{Nb}$ & 23.5 & 23.8 & 24.2 & 24.2 & 23.5 & 24.1 \\
\hline $\mathrm{Y}$ & 88.7 & 113.7 & 92.6 & 106.6 & 90.6 & 89.1 \\
\hline $\mathrm{Hf}$ & 11.6 & 11.8 & 11.9 & 12.2 & 12.0 & 11.8 \\
\hline $\mathrm{Ta}$ & 1.4 & 1.4 & 1.5 & 1.5 & 1.4 & 1.5 \\
\hline $\mathrm{U}$ & 3.5 & 3.6 & 3.4 & 3.6 & 3.5 & 3.6 \\
\hline $\mathrm{Pb}$ & 17.7 & 17.6 & 17.0 & 17.9 & 17.1 & 14.9 \\
\hline $\mathrm{Rb}$ & 73.1 & 76.2 & 88.1 & 88.9 & 81.4 & 72.8 \\
\hline Cs & 3.0 & 2.9 & 2.9 & 3.0 & 3.0 & 2.3 \\
\hline $\mathrm{Sr}$ & 34.9 & 31.3 & 34.2 & 37.2 & 30.9 & 36.8 \\
\hline $\mathrm{Sc}$ & 3.8 & 4.0 & 3.6 & 4.0 & 3.3 & 5.0 \\
\hline $\mathrm{Zr}$ & 433.6 & 437.0 & 442.0 & 457.9 & 449.2 & 445.3 \\
\hline
\end{tabular}


Table F2. Unit 2 bulk tuff major and trace element geochemical data from Streck et al., (2015).

\begin{tabular}{|c|c|c|c|c|c|c|}
\hline Sample & CR-U1ab & CR-U2a & CR-U2b-2 & CR-U2e & CR-U9b & CR-U9c \\
\hline \multicolumn{7}{|c|}{ XRF normalized (weight \%) } \\
\hline $\mathrm{SiO}_{2}$ & 74.4 & 74.7 & 74.9 & 74.9 & 71.6 & 71.8 \\
\hline $\mathrm{TiO}_{2}$ & 0.2 & 0.2 & 0.2 & 0.2 & 0.5 & 0.6 \\
\hline $\mathrm{Al}_{2} \mathrm{O}_{3}$ & 13.3 & 13.4 & 13.1 & 13.6 & 13.9 & 14.0 \\
\hline $\mathrm{FeO} *$ & 2.5 & 2.3 & 2.3 & 2.9 & 4.3 & 3.7 \\
\hline $\mathrm{MnO}$ & 0.1 & 0.1 & 0.1 & 0.1 & 0.1 & 0.1 \\
\hline $\mathrm{MgO}$ & 0.1 & 0.0 & 0.0 & 0.1 & 0.2 & 0.2 \\
\hline $\mathrm{CaO}$ & 1.0 & 0.9 & 1.0 & 1.1 & 1.7 & 1.8 \\
\hline $\mathrm{Na}_{2} \mathrm{O}$ & 3.9 & 3.0 & 3.4 & 1.9 & 4.5 & 4.7 \\
\hline $\mathrm{K}_{2} \mathrm{O}$ & 4.5 & 5.4 & 5.1 & 5.3 & 3.1 & 3.1 \\
\hline $\mathrm{P}_{2} \mathrm{O}_{5}$ & 0.0 & 0.0 & 0.0 & 0.0 & 0.2 & 0.1 \\
\hline Total & 100.0 & 100.0 & 100.0 & 100.0 & 100.0 & 100.0 \\
\hline \multicolumn{7}{|c|}{ XRF (ppm) } \\
\hline $\mathrm{Ni}$ & 0.0 & 0.0 & 0.0 & 0.0 & 0.3 & 0.2 \\
\hline $\mathrm{Cr}$ & 6.3 & 1.5 & 1.4 & 1.6 & 4.4 & 3.2 \\
\hline $\mathrm{Sc}$ & 3.5 & 2.8 & 3.3 & 3.3 & 14.1 & 13.7 \\
\hline $\mathrm{V}$ & 3.7 & 2.8 & 1.8 & 6.4 & 18.6 & 17.7 \\
\hline $\mathrm{Ba}$ & 1565.7 & 1557.0 & 1565.0 & 1568.0 & 1379.3 & 1407.1 \\
\hline $\mathrm{Rb}$ & 71.4 & 74.8 & 74.2 & 76.6 & 54.7 & 51.4 \\
\hline $\mathrm{Sr}$ & 90.5 & 89.2 & 82.0 & 89.5 & 175.1 & 178.9 \\
\hline $\mathrm{Zr}$ & 387.0 & 384.1 & 376.8 & 383.9 & 324.7 & 337.5 \\
\hline $\mathrm{Y}$ & 71.6 & 70.8 & 72.2 & 74.3 & 54.9 & 65.8 \\
\hline $\mathrm{Nb}$ & 21.0 & 20.4 & 20.9 & 19.9 & 19.7 & 20.0 \\
\hline $\mathrm{Ga}$ & 20.6 & 22.1 & 21.0 & 20.9 & 22.7 & 21.5 \\
\hline $\mathrm{Cu}$ & 2.5 & 2.5 & 1.6 & 3.3 & 3.9 & 4.2 \\
\hline $\mathrm{Zn}$ & 139.1 & 136.6 & 134.7 & 141.6 & 141.6 & 146.9 \\
\hline $\mathrm{Pb}$ & 15.0 & 13.9 & 15.2 & 15.2 & 12.4 & 11.7 \\
\hline $\mathrm{La}$ & 43.6 & 43.2 & 43.0 & 45.9 & 35.5 & 42.4 \\
\hline $\mathrm{Ce}$ & 89.5 & 85.8 & 89.3 & 95.6 & 74.7 & 74.8 \\
\hline Th & 8.3 & 9.5 & 8.6 & 9.8 & 6.8 & 7.0 \\
\hline $\mathrm{Nd}$ & 45.7 & 43.2 & 44.6 & 47.3 & 36.1 & 41.4 \\
\hline $\mathrm{U}$ & 1.8 & 2.4 & 3.2 & 2.1 & 1.9 & 2.3 \\
\hline
\end{tabular}


Table F2 continued.

\begin{tabular}{|c|c|c|c|c|c|c|}
\hline Sample & CR-U1ab & CR-U2a & CR-U2b-2 & CR-U2e & CR-U9b & CR-U9c \\
\hline \multicolumn{7}{|c|}{ ICP-MS (ppm) } \\
\hline $\mathrm{La}$ & 42.5 & 42.8 & 43.7 & 46.1 & 35.8 & 39.8 \\
\hline $\mathrm{Ce}$ & 88.1 & 88.8 & 89.9 & 96.0 & 77.5 & 78.8 \\
\hline $\operatorname{Pr}$ & 11.2 & 11.3 & 11.6 & 12.2 & 9.7 & 10.8 \\
\hline $\mathrm{Nd}$ & 44.7 & 45.3 & 46.1 & 48.9 & 38.9 & 44.4 \\
\hline $\mathrm{Sm}$ & 10.5 & 10.6 & 10.8 & 11.4 & 9.1 & 10.5 \\
\hline $\mathrm{Eu}$ & 2.0 & 2.0 & 2.1 & 2.2 & 2.5 & 2.7 \\
\hline $\mathrm{Gd}$ & 10.3 & 10.6 & 10.8 & 11.5 & 8.9 & 10.5 \\
\hline $\mathrm{Tb}$ & 1.9 & 1.9 & 2.0 & 2.1 & 1.6 & 1.9 \\
\hline Dy & 12.5 & 12.6 & 12.7 & 13.1 & 10.0 & 12.0 \\
\hline Ho & 2.7 & 2.7 & 2.7 & 2.8 & 2.1 & 2.6 \\
\hline $\mathrm{Er}$ & 7.7 & 7.7 & 7.9 & 8.1 & 6.0 & 7.3 \\
\hline $\mathrm{Tm}$ & 1.2 & 1.2 & 1.2 & 1.2 & 0.9 & 1.1 \\
\hline $\mathrm{Yb}$ & 7.4 & 7.6 & 7.6 & 7.8 & 6.0 & 7.3 \\
\hline $\mathrm{Lu}$ & 1.2 & 1.2 & 1.2 & 1.3 & 1.0 & 1.2 \\
\hline $\mathrm{Ba}$ & 1590.0 & 1568.6 & 1589.9 & 1586.5 & 1402.2 & 1427.8 \\
\hline Th & 7.1 & 7.0 & 7.0 & 7.1 & 6.0 & 6.1 \\
\hline $\mathrm{Nb}$ & 21.5 & 21.2 & 20.9 & 20.4 & 19.8 & 20.5 \\
\hline $\mathrm{Y}$ & 70.8 & 70.8 & 72.2 & 74.0 & 54.7 & 65.5 \\
\hline $\mathrm{Hf}$ & 10.2 & 10.4 & 10.1 & 10.4 & 8.6 & 8.9 \\
\hline $\mathrm{Ta}$ & 1.3 & 1.4 & 1.3 & 1.3 & 1.2 & 1.2 \\
\hline $\mathrm{U}$ & 2.9 & 3.0 & 2.9 & 2.9 & 2.5 & 2.7 \\
\hline $\mathrm{Pb}$ & 15.4 & 15.3 & 15.3 & 14.8 & 12.7 & 13.5 \\
\hline $\mathrm{Rb}$ & 73.2 & 75.8 & 74.9 & 77.3 & 56.0 & 51.6 \\
\hline Cs & 3.1 & 3.1 & 3.1 & 3.1 & 2.1 & 2.2 \\
\hline $\mathrm{Sr}$ & 89.3 & 89.3 & 81.9 & 89.4 & 177.2 & 180.5 \\
\hline Sc & 3.0 & 3.4 & 2.8 & 3.3 & 14.0 & 14.9 \\
\hline $\mathrm{Zr}$ & 404.5 & 403.8 & 394.2 & 398.4 & 337.0 & 350.0 \\
\hline
\end{tabular}


Table F2 continued.

\begin{tabular}{|c|c|c|c|c|c|c|}
\hline Sample & CR-U10a & CR-U10b & CR-U11a & $\begin{array}{c}\text { MS-PICT } \\
1-A\end{array}$ & $\begin{array}{c}\text { MS-PICT } \\
1-X 1\end{array}$ & CR-U28a \\
\hline \multicolumn{7}{|c|}{ XRF normalized (weight \%) } \\
\hline $\mathrm{SiO}_{2}$ & 72.4 & 75.4 & 73.8 & 73.8 & 74.5 & 70.7 \\
\hline $\mathrm{TiO}_{2}$ & 0.4 & 0.2 & 0.3 & 0.3 & 0.2 & 0.4 \\
\hline $\mathrm{Al}_{2} \mathrm{O}_{3}$ & 14.3 & 13.6 & 13.5 & 13.6 & 13.0 & 17.3 \\
\hline $\mathrm{FeO}^{*}$ & 3.4 & 0.8 & 2.9 & 2.9 & 2.4 & 4.3 \\
\hline $\mathrm{MnO}$ & 0.0 & 0.0 & 0.0 & 0.0 & 0.1 & 0.1 \\
\hline $\mathrm{MgO}$ & 0.2 & 0.2 & 0.1 & 0.3 & 0.0 & 0.1 \\
\hline $\mathrm{CaO}$ & 1.5 & 0.7 & 1.1 & 1.0 & 0.9 & 1.1 \\
\hline $\mathrm{Na}_{2} \mathrm{O}$ & 4.7 & 5.0 & 4.1 & 2.3 & 3.9 & 2.2 \\
\hline $\mathrm{K}_{2} \mathrm{O}$ & 2.9 & 4.1 & 4.2 & 5.6 & 4.9 & 3.9 \\
\hline $\mathrm{P}_{2} \mathrm{O}_{5}$ & 0.1 & 0.0 & 0.0 & 0.1 & 0.0 & 0.0 \\
\hline Total & 100.0 & 100.0 & 100.0 & 100.0 & 100.0 & 100.0 \\
\hline \multicolumn{7}{|c|}{ XRF (ppm) } \\
\hline $\mathrm{Ni}$ & 2.3 & 0.0 & 0.0 & 3.8 & 0.0 & 6.5 \\
\hline $\mathrm{Cr}$ & 7.0 & 3.4 & 1.9 & 4.0 & 1.6 & 10.6 \\
\hline $\mathrm{Sc}$ & 7.5 & 3.1 & 5.8 & 5.1 & 3.1 & 7.3 \\
\hline $\mathrm{V}$ & 24.7 & 0.8 & 6.1 & 10.0 & 1.4 & 28.6 \\
\hline $\mathrm{Ba}$ & 1504.8 & 1673.1 & 1662.0 & 1382.4 & 1591.1 & 1301.3 \\
\hline $\mathrm{Rb}$ & 37.0 & 74.1 & 70.0 & 79.1 & 75.7 & 62.9 \\
\hline $\mathrm{Sr}$ & 156.1 & 119.3 & 108.0 & 104.2 & 87.1 & 87.0 \\
\hline $\mathrm{Zr}$ & 356.7 & 393.0 & 376.2 & 384.3 & 384.1 & 457.6 \\
\hline $\mathrm{Y}$ & 84.9 & 86.1 & 81.3 & 76.5 & 71.3 & 69.0 \\
\hline $\mathrm{Nb}$ & 20.2 & 21.5 & 20.6 & 20.1 & 20.9 & 24.3 \\
\hline $\mathrm{Ga}$ & 22.3 & 22.4 & 22.6 & 21.5 & 22.1 & 26.7 \\
\hline $\mathrm{Cu}$ & 5.0 & 0.0 & 1.7 & 5.2 & 2.4 & 9.7 \\
\hline $\mathrm{Zn}$ & 179.6 & 57.3 & 171.6 & 84.5 & 136.7 & 137.2 \\
\hline $\mathrm{Pb}$ & 15.0 & 13.8 & 15.3 & 14.6 & 16.3 & 19.1 \\
\hline $\mathrm{La}$ & 47.6 & 50.2 & 53.1 & 47.6 & 41.1 & 43.6 \\
\hline $\mathrm{Ce}$ & 87.4 & 94.6 & 90.0 & 94.1 & 84.6 & 105.6 \\
\hline $\mathrm{Th}$ & 7.5 & 9.8 & 7.8 & 8.4 & 9.3 & 8.4 \\
\hline $\mathrm{Nd}$ & 51.0 & 50.4 & 53.6 & 46.5 & 43.4 & 49.7 \\
\hline $\mathrm{U}$ & 2.3 & 3.6 & 3.4 & 3.1 & 1.3 & 3.2 \\
\hline
\end{tabular}


Table F2 continued.

\begin{tabular}{|c|c|c|c|c|c|c|}
\hline Sample & CR-U10a & CR-U10b & CR-U11a & $\begin{array}{c}\text { MS-PICT } \\
\text { 1-A }\end{array}$ & $\begin{array}{c}\text { MS-PICT } \\
1-X 1\end{array}$ & CR-U28a \\
\hline \multicolumn{7}{|c|}{ ICP-MS (ppm) } \\
\hline $\mathrm{La}$ & 48.6 & 50.4 & 54.6 & 46.8 & 43.1 & 46.1 \\
\hline $\mathrm{Ce}$ & 89.5 & 97.9 & 91.2 & 94.9 & 88.7 & 104.6 \\
\hline $\operatorname{Pr}$ & 13.0 & 13.6 & 13.8 & 12.3 & 11.4 & 12.7 \\
\hline $\mathrm{Nd}$ & 52.9 & 54.6 & 55.3 & 49.4 & 45.7 & 50.6 \\
\hline $\mathrm{Sm}$ & 12.6 & 12.7 & 12.5 & 11.5 & 10.6 & 11.5 \\
\hline $\mathrm{Eu}$ & 2.6 & 2.3 & 2.5 & 2.2 & 2.0 & 2.3 \\
\hline $\mathrm{Gd}$ & 13.0 & 12.8 & 12.8 & 11.9 & 10.8 & 10.9 \\
\hline $\mathrm{Tb}$ & 2.4 & 2.3 & 2.3 & 2.1 & 1.9 & 1.9 \\
\hline Dy & 15.4 & 15.0 & 14.5 & 13.5 & 12.6 & 12.2 \\
\hline Ho & 3.3 & 3.2 & 3.0 & 2.9 & 2.7 & 2.6 \\
\hline $\mathrm{Er}$ & 9.4 & 9.2 & 8.6 & 8.1 & 7.7 & 7.3 \\
\hline $\mathrm{Tm}$ & 1.4 & 1.4 & 1.3 & 1.2 & 1.2 & 1.1 \\
\hline $\mathrm{Yb}$ & 9.1 & 9.1 & 8.3 & 7.8 & 7.6 & 7.0 \\
\hline $\mathrm{Lu}$ & 1.5 & 1.5 & 1.3 & 1.2 & 1.2 & 1.1 \\
\hline $\mathrm{Ba}$ & 1532.1 & 1717.8 & 1699.8 & 1400.6 & 1623.3 & 1312.3 \\
\hline $\mathrm{Th}$ & 6.7 & 7.5 & 7.0 & 7.0 & 7.1 & 8.5 \\
\hline $\mathrm{Nb}$ & 20.4 & 21.2 & 21.2 & 19.8 & 20.9 & 24.2 \\
\hline $\mathrm{Y}$ & 84.1 & 84.4 & 81.6 & 74.3 & 70.2 & 66.0 \\
\hline $\mathrm{Hf}$ & 9.5 & 10.8 & 10.0 & 10.3 & 10.3 & 11.7 \\
\hline $\mathrm{Ta}$ & 1.3 & 1.4 & 1.3 & 1.3 & 1.3 & 1.5 \\
\hline $\mathrm{U}$ & 3.0 & 3.1 & 2.9 & 3.0 & 3.0 & 2.7 \\
\hline $\mathrm{Pb}$ & 14.7 & 14.0 & 16.0 & 15.2 & 17.3 & 18.3 \\
\hline $\mathrm{Rb}$ & 38.1 & 73.8 & 70.7 & 78.5 & 75.4 & 61.1 \\
\hline Cs & 1.4 & 1.8 & 3.0 & 2.8 & 3.1 & 2.5 \\
\hline $\mathrm{Sr}$ & 157.7 & 119.3 & 109.3 & 101.2 & 86.5 & 86.0 \\
\hline $\mathrm{Sc}$ & 7.6 & 2.8 & 4.8 & 5.3 & 2.8 & 7.2 \\
\hline $\mathrm{Zr}$ & 368.2 & 412.2 & 390.5 & 396.1 & 398.8 & 459.5 \\
\hline
\end{tabular}


Table F2 continued.

\begin{tabular}{|c|c|c|c|c|c|c|}
\hline Sample & CR-U28b & CR-U28f & MS-10-1 & $\begin{array}{c}\text { MS-10-1 } \\
\text { LI1 }\end{array}$ & MS-10-20.2 & $\begin{array}{c}\text { MS-11- } \\
\text { 30drk }\end{array}$ \\
\hline \multicolumn{7}{|c|}{ XRF normalized (weight \%) } \\
\hline $\mathrm{SiO}_{2}$ & 73.2 & 73.5 & 70.5 & 74.5 & 70.1 & 74.2 \\
\hline $\mathrm{TiO}_{2}$ & 0.2 & 0.3 & 0.5 & 0.2 & 0.2 & 0.3 \\
\hline $\mathrm{Al}_{2} \mathrm{O}_{3}$ & 14.1 & 13.3 & 13.5 & 13.0 & 14.1 & 13.3 \\
\hline $\mathrm{FeO}^{*}$ & 4.0 & 3.5 & 5.0 & 2.7 & 3.0 & 2.6 \\
\hline $\mathrm{MnO}$ & 0.1 & 0.1 & 0.1 & 0.1 & 0.1 & 0.1 \\
\hline $\mathrm{MgO}$ & 0.1 & 0.1 & 1.1 & 0.1 & 0.5 & 0.3 \\
\hline $\mathrm{CaO}$ & 0.9 & 1.0 & 2.3 & 1.0 & 4.4 & 1.0 \\
\hline $\mathrm{Na}_{2} \mathrm{O}$ & 1.9 & 2.6 & 3.4 & 3.7 & 3.1 & 2.7 \\
\hline $\mathrm{K}_{2} \mathrm{O}$ & 5.5 & 5.6 & 3.4 & 4.7 & 4.4 & 5.6 \\
\hline $\mathrm{P}_{2} \mathrm{O}_{5}$ & 0.0 & 0.0 & 0.1 & 0.0 & 0.0 & 0.0 \\
\hline Total & 100.0 & 100.0 & 100.0 & 100.0 & 100.0 & 100.0 \\
\hline \multicolumn{7}{|c|}{ XRF (ppm) } \\
\hline $\mathrm{Ni}$ & 2.8 & 1.2 & 8.4 & 1.8 & 0.0 & 2.8 \\
\hline $\mathrm{Cr}$ & 3.7 & 10.1 & 19.8 & 2.5 & 2.9 & 4.4 \\
\hline $\mathrm{Sc}$ & 4.0 & 6.0 & 13.1 & 4.3 & 6.3 & 3.6 \\
\hline $\mathrm{V}$ & 14.6 & 8.8 & 32.9 & 3.3 & 5.7 & 8.2 \\
\hline $\mathrm{Ba}$ & 1483.9 & 1710.0 & 1188.3 & 1539.1 & 1102.2 & 1445.1 \\
\hline $\mathrm{Rb}$ & 93.2 & 75.2 & 62.7 & 74.8 & 72.2 & 76.4 \\
\hline $\mathrm{Sr}$ & 83.9 & 100.6 & 189.4 & 87.4 & 269.6 & 93.8 \\
\hline $\mathrm{Zr}$ & 418.8 & 392.1 & 308.1 & 390.5 & 317.9 & 390.4 \\
\hline $\mathrm{Y}$ & 67.3 & 69.5 & 59.4 & 70.8 & 48.6 & 73.4 \\
\hline $\mathrm{Nb}$ & 21.9 & 21.2 & 17.8 & 21.9 & 12.4 & 21.7 \\
\hline $\mathrm{Ga}$ & 23.4 & 22.3 & 20.7 & 21.7 & 19.7 & 21.2 \\
\hline $\mathrm{Cu}$ & 6.8 & 3.9 & 9.6 & 2.8 & 3.2 & 5.5 \\
\hline $\mathrm{Zn}$ & 165.2 & 122.8 & 143.6 & 128.9 & 144.7 & 134.3 \\
\hline $\mathrm{Pb}$ & 15.6 & 25.6 & 13.4 & 13.4 & 9.0 & 15.8 \\
\hline $\mathrm{La}$ & 38.9 & 40.3 & 33.4 & 43.4 & 25.0 & 44.5 \\
\hline $\mathrm{Ce}$ & 83.0 & 85.4 & 68.9 & 81.9 & 47.9 & 90.7 \\
\hline Th & 8.7 & 7.6 & 5.2 & 7.2 & 6.9 & 6.4 \\
\hline $\mathrm{Nd}$ & 42.2 & 42.5 & 39.5 & 40.7 & 29.1 & 44.6 \\
\hline $\mathrm{U}$ & 2.7 & 3.2 & 2.3 & 3.1 & 1.9 & 4.1 \\
\hline
\end{tabular}


Table F2 continued.

\begin{tabular}{|c|c|c|c|c|c|c|}
\hline Sample & CR-U28b & CR-U28f & MS-10-1 & $\begin{array}{c}\text { MS-10-1 } \\
\text { LI1 }\end{array}$ & $\begin{array}{c}\text { MS-10- } \\
20.2\end{array}$ & $\begin{array}{r}\text { MS-11 } \\
\text { 30drk }\end{array}$ \\
\hline \multicolumn{7}{|c|}{ ICP-MS (ppm) } \\
\hline $\mathrm{La}$ & 41.0 & 40.5 & 34.3 & 41.7 & 29.3 & 43.9 \\
\hline $\mathrm{Ce}$ & 85.6 & 82.1 & 70.8 & 86.7 & 57.3 & 90.7 \\
\hline $\operatorname{Pr}$ & 11.0 & 10.7 & 9.3 & 11.0 & 8.0 & 11.6 \\
\hline $\mathrm{Nd}$ & 44.2 & 42.3 & 37.8 & 43.8 & 32.0 & 46.7 \\
\hline $\mathrm{Sm}$ & 10.3 & 10.0 & 8.9 & 10.1 & 7.4 & 10.9 \\
\hline $\mathrm{Eu}$ & 1.7 & 1.8 & 2.1 & 1.9 & 1.4 & 2.2 \\
\hline $\mathrm{Gd}$ & 10.3 & 10.3 & 9.2 & 10.4 & 7.7 & 11.1 \\
\hline $\mathrm{Tb}$ & 1.8 & 1.8 & 1.6 & 1.9 & 1.4 & 2.0 \\
\hline Dy & 11.9 & 12.0 & 10.5 & 12.2 & 9.1 & 12.8 \\
\hline Ho & 2.5 & 2.6 & 2.2 & 2.6 & 2.0 & 2.8 \\
\hline $\mathrm{Er}$ & 7.2 & 7.4 & 6.3 & 7.6 & 5.6 & 7.8 \\
\hline $\mathrm{Tm}$ & 1.1 & 1.1 & 0.9 & 1.1 & 0.9 & 1.2 \\
\hline $\mathrm{Yb}$ & 7.0 & 7.1 & 6.0 & 7.4 & 5.5 & 7.6 \\
\hline $\mathrm{Lu}$ & 1.1 & 1.2 & 1.0 & 1.2 & 0.9 & 1.2 \\
\hline $\mathrm{Ba}$ & 1512.4 & 1741.0 & 1226.0 & 1610.9 & 1228.4 & 1483.5 \\
\hline Th & 7.6 & 7.1 & 5.7 & 7.1 & 7.3 & 7.2 \\
\hline $\mathrm{Nb}$ & 21.3 & 21.1 & 17.2 & 20.4 & 12.6 & 21.1 \\
\hline $\mathrm{Y}$ & 65.4 & 67.2 & 56.7 & 67.3 & 50.8 & 71.9 \\
\hline $\mathrm{Hf}$ & 10.7 & 10.2 & 8.0 & 10.2 & 9.1 & 10.3 \\
\hline $\mathrm{Ta}$ & 1.4 & 1.3 & 1.1 & 1.3 & 0.9 & 1.3 \\
\hline $\mathrm{U}$ & 2.8 & 2.9 & 2.4 & 3.0 & 3.1 & 3.0 \\
\hline $\mathrm{Pb}$ & 14.8 & 25.5 & 12.1 & 11.8 & 7.6 & 15.4 \\
\hline $\mathrm{Rb}$ & 91.3 & 74.4 & 60.8 & 74.7 & 76.7 & 76.3 \\
\hline Cs & 3.1 & 3.0 & 2.4 & 3.0 & 3.9 & 3.2 \\
\hline $\mathrm{Sr}$ & 84.8 & 99.6 & 187.7 & 86.5 & 279.9 & 96.8 \\
\hline $\mathrm{Sc}$ & 4.0 & 5.8 & 13.9 & 4.3 & 6.7 & 4.0 \\
\hline $\mathrm{Zr}$ & 424.9 & 399.0 & 317.9 & 404.1 & 351.7 & 424.7 \\
\hline
\end{tabular}


Table F2 continued.

\begin{tabular}{|c|c|c|c|c|c|}
\hline Sample & MS-11-30 lit & MS-11-32 & MS 14-24b* & MS15-21A* & MS15-25A \\
\hline \multicolumn{6}{|c|}{ XRF normalized (normalized weight \%) } \\
\hline $\mathrm{SiO}_{2}$ & 74.5 & 75.1 & 75.1 & 74.2 & 75.6 \\
\hline $\mathrm{TiO}_{2}$ & 0.2 & 0.2 & 0.2 & 0.2 & 0.2 \\
\hline $\mathrm{Al}_{2} \mathrm{O}_{3}$ & 13.3 & 13.4 & 12.6 & 12.9 & 13.3 \\
\hline $\mathrm{FeO}^{*}$ & 2.7 & 2.2 & 3.2 & 2.7 & 1.5 \\
\hline $\mathrm{MnO}$ & 0.1 & 0.0 & 0.0 & 0.1 & 0.0 \\
\hline $\mathrm{MgO}$ & 0.2 & 0.0 & 0.1 & 0.1 & 0.1 \\
\hline $\mathrm{CaO}$ & 1.0 & 0.8 & 0.6 & 0.9 & 0.4 \\
\hline $\mathrm{Na}_{2} \mathrm{O}$ & 3.1 & 3.2 & 4.5 & 3.0 & 4.7 \\
\hline $\mathrm{K}_{2} \mathrm{O}$ & 5.0 & 4.9 & 3.7 & 6.0 & 4.1 \\
\hline $\mathrm{P}_{2} \mathrm{O}_{5}$ & 0.0 & 0.0 & 0.0 & 0.0 & 0.0 \\
\hline Total & 100.0 & 100.0 & 99.0 & 95.4 & 98.1 \\
\hline \multicolumn{6}{|c|}{$\mathrm{XRF}(\mathrm{ppm})$} \\
\hline $\mathrm{Ni}$ & 1.3 & 1.8 & 4.5 & 3.8 & 2.4 \\
\hline $\mathrm{Cr}$ & 3.8 & 3.3 & 3.9 & 1.2 & 2.0 \\
\hline Sc & 4.6 & 2.9 & 3.1 & 2.3 & 2.7 \\
\hline V & 5.1 & 2.8 & 3.8 & 1.9 & 6.0 \\
\hline $\mathrm{Ba}$ & 1452.3 & 1559.3 & 1574.2 & 1566.9 & 1665.4 \\
\hline $\mathrm{Rb}$ & 78.9 & 72.3 & 74.3 & 84.0 & 73.3 \\
\hline $\mathrm{Sr}$ & 100.1 & 87.9 & 88.8 & 93.5 & 89.8 \\
\hline $\mathrm{Zr}$ & 391.3 & 397.8 & 392.9 & 391.8 & 423.2 \\
\hline Y & 70.2 & 72.9 & 55.6 & 72.4 & 49.7 \\
\hline $\mathrm{Nb}$ & 22.3 & 21.6 & 22.1 & 21.3 & 23.1 \\
\hline $\mathrm{Ga}$ & 22.9 & 22.5 & 22.2 & 21.5 & 22.7 \\
\hline $\mathrm{Cu}$ & 4.4 & 2.7 & 2.9 & 3.3 & 2.0 \\
\hline $\mathrm{Zn}$ & 135.3 & 128.7 & 115.0 & 137.1 & 113.4 \\
\hline $\mathrm{Pb}$ & 16.1 & 17.3 & 12.5 & 15.8 & 14.8 \\
\hline $\mathrm{La}$ & 43.3 & 41.3 & 38.8 & 41.1 & 30.8 \\
\hline $\mathrm{Ce}$ & 88.2 & 86.9 & 74.5 & 88.6 & 64.0 \\
\hline Th & 7.2 & 6.8 & 7.2 & 7.6 & 7.8 \\
\hline $\mathrm{Nd}$ & 46.5 & 42.8 & 40.6 & 43.3 & 36.7 \\
\hline $\mathrm{U}$ & 2.6 & 4.0 & 2.5 & 3.3 & 1.5 \\
\hline
\end{tabular}


Table F2 continued.

\begin{tabular}{|c|c|c|c|c|c|}
\hline Sample & MS-11-30 lit & MS-11-32 & MS 14-24b & MS15-21A & MS15-25A \\
\hline \multicolumn{6}{|c|}{ ICP-MS (ppm) } \\
\hline $\mathrm{La}$ & 44.5 & 44.3 & 39.3 & 43.9 & 37.2 \\
\hline $\mathrm{Ce}$ & 89.9 & 88.9 & 77.1 & 90.4 & 67.5 \\
\hline $\operatorname{Pr}$ & 11.8 & 11.8 & 10.5 & 11.6 & 9.8 \\
\hline $\mathrm{Nd}$ & 47.0 & 47.2 & 41.2 & 46.4 & 39.3 \\
\hline $\mathrm{Sm}$ & 10.9 & 10.9 & 9.6 & 10.6 & 9.0 \\
\hline $\mathrm{Eu}$ & 2.2 & 2.1 & 2.1 & 2.1 & 2.2 \\
\hline $\mathrm{Gd}$ & 11.0 & 10.8 & 9.1 & 10.9 & 8.4 \\
\hline $\mathrm{Tb}$ & 2.0 & 2.0 & 1.6 & 2.0 & 1.5 \\
\hline Dy & 12.6 & 12.9 & 10.3 & 12.7 & 9.5 \\
\hline Ho & 2.7 & 2.7 & 2.2 & 2.7 & 1.9 \\
\hline $\mathrm{Er}$ & 7.6 & 7.9 & 6.3 & 7.9 & 5.5 \\
\hline $\mathrm{Tm}$ & 1.1 & 1.2 & 1.0 & 1.2 & 0.8 \\
\hline $\mathrm{Yb}$ & 7.3 & 7.6 & 6.2 & 7.7 & 5.3 \\
\hline $\mathrm{Lu}$ & 1.2 & 1.2 & 0.9 & 1.2 & 0.8 \\
\hline $\mathrm{Ba}$ & 1484.8 & 1587.8 & 1608.4 & 1632.8 & 1733.6 \\
\hline Th & 7.1 & 7.2 & 7.2 & 7.3 & 7.7 \\
\hline $\mathrm{Nb}$ & 20.1 & 20.4 & 20.5 & 21.4 & 22.8 \\
\hline $\mathrm{Y}$ & 68.7 & 71.6 & 53.3 & 71.7 & 49.1 \\
\hline $\mathrm{Hf}$ & 10.4 & 10.6 & 10.3 & 10.4 & 11.2 \\
\hline $\mathrm{Ta}$ & 1.3 & 1.3 & 1.3 & 1.4 & 1.4 \\
\hline $\mathrm{U}$ & 2.8 & 3.0 & 2.8 & 3.0 & 2.4 \\
\hline $\mathrm{Pb}$ & 15.0 & 15.7 & 12.1 & 15.7 & 14.3 \\
\hline $\mathrm{Rb}$ & 79.7 & 72.3 & 74.1 & 81.9 & 71.9 \\
\hline Cs & 3.3 & 3.0 & 2.6 & 3.3 & 1.5 \\
\hline $\mathrm{Sr}$ & 103.7 & 91.3 & 90.0 & 95.7 & 94.5 \\
\hline Sc & 4.0 & 3.0 & 2.8 & 2.9 & 2.8 \\
\hline $\mathrm{Zr}$ & 418.9 & 433.6 & 395.7 & 402.1 & 433.5 \\
\hline
\end{tabular}


Table F3. Unit 3 bulk tuff major and trace element geochemical data from Streck et al. (2015) and Streck pers. comm. (2017).

\begin{tabular}{|c|c|c|c|c|}
\hline Sample & MS-10-26 & MS-10-BCT1 & MS-U35a & MS12-33.1 \\
\hline \multicolumn{5}{|c|}{ XRF normalized (weight \%) } \\
\hline $\mathrm{SiO}_{2}$ & 76.6 & 71.2 & 74.1 & 75.1 \\
\hline $\mathrm{TiO}_{2}$ & 0.2 & 0.7 & 0.3 & 0.4 \\
\hline $\mathrm{Al}_{2} \mathrm{O}_{3}$ & 12.1 & 13.3 & 13.2 & 13.1 \\
\hline $\mathrm{FeO}^{*}$ & 1.6 & 4.8 & 2.6 & 3.6 \\
\hline $\mathrm{MnO}$ & 0.1 & 0.1 & 0.1 & 0.1 \\
\hline $\mathrm{MgO}$ & 0.1 & 1.2 & 0.1 & 0.1 \\
\hline $\mathrm{CaO}$ & 0.4 & 1.8 & 0.5 & 0.8 \\
\hline $\mathrm{Na}_{2} \mathrm{O}$ & 3.3 & 2.7 & 3.2 & 1.9 \\
\hline $\mathrm{K}_{2} \mathrm{O}$ & 5.7 & 3.9 & 6.1 & 4.8 \\
\hline $\mathrm{P}_{2} \mathrm{O}_{5}$ & 0.0 & 0.3 & 0.0 & 0.0 \\
\hline Total & 100.0 & 100.0 & 100.0 & 100.0 \\
\hline \multicolumn{5}{|c|}{ XRF unnormalized (ppm) } \\
\hline $\mathrm{Ni}$ & 2.0 & 8.0 & 4.0 & 4.1 \\
\hline $\mathrm{Cr}$ & 4.0 & 14.6 & 3.4 & 5.9 \\
\hline $\mathrm{Sc}$ & 5.0 & 12.5 & 1.7 & 5.2 \\
\hline V & 6.0 & 52.8 & 6.3 & 18.4 \\
\hline $\mathrm{Ba}$ & 797.0 & 816.2 & 1815.4 & 1168.4 \\
\hline $\mathrm{Rb}$ & 89.0 & 71.1 & 84.6 & 78.4 \\
\hline $\mathrm{Sr}$ & 16.0 & 91.8 & 54.4 & 47.1 \\
\hline $\mathrm{Zr}$ & 306.0 & 341.3 & 390.2 & 372.6 \\
\hline $\mathrm{Y}$ & 88.0 & 57.4 & 62.1 & 61.5 \\
\hline $\mathrm{Nb}$ & 27.5 & 22.7 & 24.3 & 23.0 \\
\hline $\mathrm{Ga}$ & 20.0 & 18.9 & 22.0 & 20.3 \\
\hline $\mathrm{Cu}$ & 1.0 & 11.4 & 2.9 & 3.9 \\
\hline $\mathrm{Zn}$ & 108.0 & 123.2 & 149.1 & 138.9 \\
\hline $\mathrm{Pb}$ & 18.0 & 14.9 & 15.1 & 15.4 \\
\hline $\mathrm{La}$ & 41.0 & 38.9 & 44.6 & 40.1 \\
\hline $\mathrm{Ce}$ & 91.0 & 84.1 & 82.8 & 85.1 \\
\hline $\mathrm{Th}$ & 8.0 & 5.6 & 6.6 & 7.3 \\
\hline $\mathrm{Nd}$ & 49.0 & 40.9 & 43.7 & 43.1 \\
\hline $\mathrm{U}$ & 3.0 & 1.6 & 1.8 & 3.7 \\
\hline
\end{tabular}


Table F3 continued.

\begin{tabular}{|c|c|c|c|c|}
\hline Sample & MS-10-26 & MS-10-BCT1 & MS-U35a & MS12-33.1 \\
\hline \multicolumn{5}{|c|}{ ICP-MS (ppm) } \\
\hline $\mathrm{La}$ & 41.8 & 41.4 & 46.3 & 39.6 \\
\hline $\mathrm{Ce}$ & 95.5 & 85.0 & 92.0 & 82.9 \\
\hline $\operatorname{Pr}$ & 12.5 & 10.8 & 11.7 & 10.4 \\
\hline $\mathrm{Nd}$ & 50.1 & 42.2 & 46.2 & 41.2 \\
\hline $\mathrm{Sm}$ & 13.1 & 9.4 & 10.3 & 9.3 \\
\hline $\mathrm{Eu}$ & 1.7 & 1.9 & 1.9 & 1.6 \\
\hline $\mathrm{Gd}$ & 13.3 & 9.1 & 9.9 & 8.9 \\
\hline $\mathrm{Tb}$ & 2.5 & 1.6 & 1.8 & 1.6 \\
\hline Dy & 15.8 & 10.1 & 11.2 & 10.5 \\
\hline Ho & 3.4 & 2.2 & 2.4 & 2.2 \\
\hline $\mathrm{Er}$ & 9.4 & 6.0 & 6.7 & 6.3 \\
\hline $\mathrm{Tm}$ & 1.4 & 0.9 & 1.0 & 1.0 \\
\hline $\mathrm{Yb}$ & 9.1 & 5.9 & 6.7 & 6.2 \\
\hline $\mathrm{Lu}$ & 1.4 & 1.0 & 1.1 & 1.0 \\
\hline $\mathrm{Ba}$ & 825.0 & 835.3 & 1985.3 & 1175.2 \\
\hline Th & 7.7 & 6.6 & 7.4 & 7.1 \\
\hline $\mathrm{Nb}$ & 28.4 & 22.0 & 23.9 & 22.1 \\
\hline $\mathrm{Y}$ & 86.2 & 56.5 & 62.0 & 58.4 \\
\hline $\mathrm{Hf}$ & 9.3 & 8.7 & 10.3 & 9.6 \\
\hline $\mathrm{Ta}$ & 1.8 & 1.3 & 1.3 & 1.4 \\
\hline $\mathrm{U}$ & 3.3 & 2.6 & 3.3 & 4.3 \\
\hline $\mathrm{Pb}$ & 17.4 & 12.5 & 15.2 & 13.8 \\
\hline $\mathrm{Rb}$ & 91.0 & 72.0 & 88.6 & 77.8 \\
\hline Cs & 3.2 & 2.6 & 2.9 & 3.6 \\
\hline $\mathrm{Sr}$ & 16.0 & 91.4 & 52.8 & 49.0 \\
\hline $\mathrm{Sc}$ & 6.0 & 13.2 & 4.8 & 4.6 \\
\hline $\mathrm{Zr}$ & 328.0 & 355.8 & 423.9 & 374.7 \\
\hline
\end{tabular}


Table F3 continued.

\begin{tabular}{lrrr}
\hline \hline Sample & MS-12-38 & CR-U33c & EBC12-074* \\
\hline \multicolumn{2}{l}{$\mathrm{XRF}$ normalized (weight $\%)$} & & \\
\hline $\mathrm{SiO}_{2}$ & 73.3 & 72.2 & 75.0 \\
$\mathrm{TiO}_{2}$ & 0.6 & 0.2 & 0.2 \\
$\mathrm{Al}_{2} \mathrm{O}_{3}$ & 13.7 & 14.2 & 12.8 \\
$\mathrm{FeO}^{*}$ & 4.1 & 2.9 & 2.6 \\
$\mathrm{MnO}$ & 0.1 & 0.1 & 0.0 \\
$\mathrm{MgO}$ & 0.4 & 0.6 & 0.0 \\
$\mathrm{CaO}$ & 1.2 & 2.1 & 0.3 \\
$\mathrm{Na} 2 \mathrm{O}$ & 1.4 & 3.3 & 2.5 \\
$\mathrm{~K}_{2} \mathrm{O}$ & 4.9 & 4.3 & 6.5 \\
$\mathrm{P}_{2} \mathrm{O}_{5}$ & 0.1 & 0.1 & 0.0 \\
\hline $\mathrm{Total}$ & 100.0 & 100.0 & 94.0 \\
\hline $\mathrm{XRF}$ unnormalized $(\mathrm{ppm})$ & & \\
$\mathrm{Ni}$ & 7.4 & 1.1 & N/A \\
$\mathrm{Cr}$ & 9.1 & 1.7 & N/A \\
$\mathrm{Sc}$ & 9.9 & 6.0 & N/A \\
$\mathrm{V}$ & 40.6 & 6.8 & N/A \\
$\mathrm{Ba}$ & 784.0 & 1083.7 & N/A \\
$\mathrm{Rb}$ & 122.4 & 77.0 & N/A \\
$\mathrm{Sr}$ & 71.4 & 122.2 & N/A \\
$\mathrm{Zr}$ & 373.4 & 348.6 & N/A \\
$\mathrm{Y}$ & 55.6 & 61.8 & N/A \\
$\mathrm{Nb}$ & 23.6 & 14.0 & N/A \\
$\mathrm{Ga}$ & 21.2 & 20.8 & N/A \\
$\mathrm{Cu}$ & 12.1 & 2.9 & N/A \\
$\mathrm{Zn}$ & 120.2 & 148.4 & N/A \\
$\mathrm{Pb}$ & 15.0 & 13.5 & N/A \\
$\mathrm{La}$ & 41.0 & 27.8 & N/A \\
$\mathrm{Ce}$ & 83.5 & 65.5 & N/A \\
$\mathrm{Th}$ & 6.9 & 7.0 & N/A \\
$\mathrm{Nd}$ & 40.1 & 34.9 & N/A \\
$\mathrm{U}$ & 3.1 & 2.9 & N/A \\
\hline & & &
\end{tabular}


Table F3 continued.

\begin{tabular}{|c|c|c|c|}
\hline Sample & MS-12-38 & CR-U33c & EBC12-074* \\
\hline \multicolumn{3}{|c|}{ ICP-MS (ppm) } & \\
\hline $\mathrm{La}$ & 41.4 & 33.0 & N/A \\
\hline $\mathrm{Ce}$ & 84.9 & 67.8 & N/A \\
\hline $\operatorname{Pr}$ & 10.7 & 9.0 & N/A \\
\hline $\mathrm{Nd}$ & 41.8 & 36.8 & N/A \\
\hline $\mathrm{Sm}$ & 9.2 & 8.7 & N/A \\
\hline $\mathrm{Eu}$ & 1.7 & 1.4 & N/A \\
\hline $\mathrm{Gd}$ & 8.7 & 9.0 & N/A \\
\hline $\mathrm{Tb}$ & 1.5 & 1.6 & N/A \\
\hline Dy & 9.9 & 10.5 & N/A \\
\hline Ho & 2.1 & 2.3 & N/A \\
\hline $\mathrm{Er}$ & 5.8 & 6.5 & N/A \\
\hline $\mathrm{Tm}$ & 0.9 & 1.0 & N/A \\
\hline $\mathrm{Yb}$ & 5.8 & 6.4 & N/A \\
\hline $\mathrm{Lu}$ & 0.9 & 1.0 & N/A \\
\hline $\mathrm{Ba}$ & 781.6 & 1105.2 & N/A \\
\hline Th & 7.3 & 6.9 & N/A \\
\hline $\mathrm{Nb}$ & 22.7 & 13.6 & N/A \\
\hline $\mathrm{Y}$ & 53.0 & 60.0 & N/A \\
\hline $\mathrm{Hf}$ & 9.4 & 9.3 & N/A \\
\hline $\mathrm{Ta}$ & 1.3 & 0.9 & N/A \\
\hline $\mathrm{U}$ & 2.8 & 3.4 & N/A \\
\hline $\mathrm{Pb}$ & 14.2 & 12.7 & N/A \\
\hline $\mathrm{Rb}$ & 118.2 & 76.9 & N/A \\
\hline Cs & 3.5 & 3.4 & N/A \\
\hline $\mathrm{Sr}$ & 73.0 & 121.7 & N/A \\
\hline $\mathrm{Sc}$ & 10.3 & 6.6 & N/A \\
\hline $\mathrm{Zr}$ & 370.5 & 366.7 & N/A \\
\hline
\end{tabular}


Table F4. Unit 4 bulk tuff major and trace element geochemical data from Streck et al., (2015).

\begin{tabular}{|c|c|c|c|c|c|}
\hline Sample & MS-11-11 & MS-11-20 & MS-12-20* & MS-15-20A* & $\overline{M S-10-20.1}$ \\
\hline \multicolumn{6}{|c|}{ XRF normalized (weight \%) } \\
\hline $\mathrm{SiO}_{2}$ & 66.6 & 68.0 & 67.0 & 69.0 & 70.0 \\
\hline $\mathrm{TiO}_{2}$ & 1.2 & 1.1 & 1.2 & 0.7 & 0.4 \\
\hline $\mathrm{Al}_{2} \mathrm{O}_{3}$ & 14.8 & 14.5 & 14.7 & 14.0 & 14.5 \\
\hline $\mathrm{FeO}^{*}$ & 7.1 & 5.7 & 6.2 & 4.8 & 4.9 \\
\hline $\mathrm{MnO}$ & 0.2 & 0.1 & 0.2 & 0.1 & 0.1 \\
\hline $\mathrm{MgO}$ & 1.6 & 1.1 & 0.9 & 1.1 & 0.4 \\
\hline $\mathrm{CaO}$ & 3.0 & 3.0 & 3.2 & 2.3 & 2.7 \\
\hline $\mathrm{Na}_{2} \mathrm{O}$ & 2.5 & 3.1 & 2.5 & 4.0 & 2.5 \\
\hline $\mathrm{K}_{2} \mathrm{O}$ & 2.7 & 3.0 & 3.4 & 3.7 & 4.4 \\
\hline $\mathrm{P}_{2} \mathrm{O}_{5}$ & 0.4 & 0.4 & 0.7 & 0.3 & 0.2 \\
\hline Total & 100.0 & 100.0 & 93.3 & 95.3 & 100.0 \\
\hline \multicolumn{6}{|c|}{ XRF unnormalized (ppm) } \\
\hline $\mathrm{Ni}$ & 6.0 & 3.6 & 4.1 & 3.1 & 1.2 \\
\hline $\mathrm{Cr}$ & 12.5 & 6.9 & 5.6 & 1.1 & 4.2 \\
\hline Sc & 17.3 & 16.4 & 17.2 & 11.0 & 12.3 \\
\hline $\mathrm{V}$ & 84.6 & 68.7 & 67.5 & 46.0 & 10.1 \\
\hline $\mathrm{Ba}$ & 1026.5 & 1296.4 & 1234.6 & 1485.5 & 1289.0 \\
\hline $\mathrm{Rb}$ & 47.8 & 54.1 & 59.6 & 59.2 & 75.0 \\
\hline $\mathrm{Sr}$ & 207.8 & 233.6 & 234.7 & 176.1 & 227.4 \\
\hline $\mathrm{Zr}$ & 255.2 & 272.4 & 277.6 & 317.6 & 294.3 \\
\hline $\mathrm{Y}$ & 48.4 & 50.5 & 52.1 & 60.0 & 57.3 \\
\hline $\mathrm{Nb}$ & 16.2 & 17.7 & 18.2 & 20.6 & 14.4 \\
\hline $\mathrm{Ga}$ & 19.9 & 21.3 & 19.7 & 20.4 & 21.5 \\
\hline $\mathrm{Cu}$ & 9.6 & 6.6 & 11.4 & 4.7 & 5.4 \\
\hline $\mathrm{Zn}$ & 139.5 & 140.8 & 141.9 & 147.2 & 175.8 \\
\hline $\mathrm{Pb}$ & 10.0 & 10.6 & 11.4 & 11.8 & 12.6 \\
\hline $\mathrm{La}$ & 28.6 & 33.7 & 32.2 & 38.3 & 29.1 \\
\hline $\mathrm{Ce}$ & 70.2 & 67.7 & 71.8 & 72.3 & 59.4 \\
\hline Th & 4.7 & 5.6 & 5.8 & 5.8 & 6.1 \\
\hline $\mathrm{Nd}$ & 38.1 & 39.0 & 39.2 & 42.0 & 31.9 \\
\hline $\mathrm{U}$ & 2.9 & 1.8 & 2.5 & 2.6 & 1.8 \\
\hline
\end{tabular}


Table F4 continued.

\begin{tabular}{|c|c|c|c|c|c|}
\hline Sample & MS-11-11 & MS-11-20 & MS-12-20 & MS-15-20A & MS-10-20.1 \\
\hline \multicolumn{6}{|c|}{ ICP-MS (ppm) } \\
\hline $\mathrm{La}$ & 30.6 & 33.5 & 34.3 & 39.8 & 29.1 \\
\hline $\mathrm{Ce}$ & 65.2 & 69.9 & 71.6 & 79.6 & 59.2 \\
\hline $\operatorname{Pr}$ & 8.6 & 9.1 & 9.6 & 10.6 & 8.0 \\
\hline $\mathrm{Nd}$ & 35.8 & 37.9 & 39.4 & 42.9 & 32.4 \\
\hline $\mathrm{Sm}$ & 8.2 & 8.8 & 9.0 & 9.8 & 7.8 \\
\hline $\mathrm{Eu}$ & 2.3 & 2.4 & 2.4 & 2.5 & 1.7 \\
\hline $\mathrm{Gd}$ & 8.3 & 8.8 & 9.0 & 9.7 & 8.2 \\
\hline $\mathrm{Tb}$ & 1.4 & 1.5 & 1.5 & 1.7 & 1.5 \\
\hline Dy & 8.7 & 9.2 & 9.4 & 10.6 & 9.7 \\
\hline Ho & 1.8 & 1.9 & 1.9 & 2.2 & 2.1 \\
\hline $\mathrm{Er}$ & 5.0 & 5.3 & 5.4 & 6.3 & 6.0 \\
\hline $\mathrm{Tm}$ & 0.7 & 0.8 & 0.8 & 1.0 & 0.9 \\
\hline $\mathrm{Yb}$ & 4.6 & 5.0 & 5.1 & 6.1 & 5.8 \\
\hline $\mathrm{Lu}$ & 0.8 & 0.8 & 0.8 & 1.0 & 1.0 \\
\hline $\mathrm{Ba}$ & 1021.3 & 1319.0 & 1234.1 & 1519.5 & 1330.8 \\
\hline Th & 4.9 & 5.3 & 5.4 & 6.2 & 6.7 \\
\hline $\mathrm{Nb}$ & 15.7 & 17.3 & 17.4 & 20.4 & 13.5 \\
\hline $\mathrm{Y}$ & 46.8 & 50.1 & 50.6 & 59.5 & 55.5 \\
\hline $\mathrm{Hf}$ & 6.4 & 7.1 & 7.1 & 8.4 & 7.8 \\
\hline $\mathrm{Ta}$ & 1.0 & 1.1 & 1.1 & 1.3 & 0.8 \\
\hline $\mathrm{U}$ & 2.2 & 2.5 & 2.4 & 2.9 & 2.8 \\
\hline $\mathrm{Pb}$ & 9.7 & 10.6 & 11.5 & 12.3 & 12.0 \\
\hline $\mathrm{Rb}$ & 45.9 & 53.7 & 57.3 & 57.2 & 75.1 \\
\hline Cs & 2.1 & 2.3 & 2.4 & 2.0 & 3.6 \\
\hline $\mathrm{Sr}$ & 211.4 & 239.3 & 239.7 & 181.0 & 226.3 \\
\hline Sc & 17.2 & 16.5 & 16.4 & 11.0 & 12.4 \\
\hline $\mathrm{Zr}$ & 251.0 & 278.2 & 273.4 & 327.2 & 307.5 \\
\hline
\end{tabular}

